\title{
Spatial and temporal regulation of cytokine expression in Type 2 immune responses
}

\author{
By \\ Ryan Luke Kyle
}

A thesis submitted to the Victoria University of Wellington in fulfilment of the requirements for the degree of Doctor of Philosophy in Science

Victoria University 



\section{Abstract}

Type 2 immune responses are generated to provide protection against parasitic helminth infections, however these responses also cause the pathologies associated with allergic inflammation. Studies of the cell types and signalling pathways that mediate Type 2 immune responses have been previously undertaken with the goals of efficient development of vaccines against helminths, and identification of pathways that can be inhibited to decrease the damage caused by allergic inflammation.

The cytokines interleukin-4 (IL-4) and interleukin-13 (IL-13) mediate many of the downstream effector functions of the Type 2 immune response. To study the mechanisms that control expression of these two cytokines I have used a novel dual cytokine IL-4 and IL-13 transgenic reporter mouse. Utilising this tool along with other IL-4 reporter mice I have discovered that the amount of $\mathrm{T}$ cell receptor (TCR) signalling modulates the allelic expression of IL- 4 by CD4 ${ }^{+} \mathrm{T}$ cells. The transgenic IL-4 reporter mouse has for the first time allowed independent measurement of the effects of IL-4 deficiency on the expression of IL-4 in vivo. Using this system I have found that IL4 is not required for the in vivo generation or expansion of IL-4 producing CD4 ${ }^{+} \mathrm{T}$ cells. Th2 differentiated CD4+ T cells also expresses IL-13, however the dual reporter mice have demonstrated that IL-13 is expressed consistently later than IL-4 in vitro, and IL-13 requires constant, or multiple exposures to TCR stimulus for expression to be induced. IL-13 expression is absent from lymph node $\mathrm{CD} 4^{+} \mathrm{T}$ cells during exposure to allergens or helminth infection. Sequestration of $\mathrm{CD}^{+} \mathrm{T}$ cells in the lymph node does not impact the number of IL-13 expressing CD4+ $\mathrm{T}$ cells in the lung during a helminth infection, 
indicating that adaptive immune cell derived IL-13 may be entirely produced by lung resident cells not requiring transit through the lymph node.

I have characterised a population of innate lymphoid cells (ILCs) within the skin and found that the proportion of these cells that constitutively express IL-13 decreases with age. These cells did not drastically change in numbers or IL-13 responses in a range of inflammatory conditions including a model of atopic dermatitis. Basophils were found to respond to the atopic dermatitis model by migrating specifically to the treated skin site and draining lymph node, and producing IL-4 in a thymic stromal lymphopoietin dependant manner.

Treatment with exogenous cytokines induced IL-13 expression from group 2 ILCs (ILC2s) in the lung and these cells promoted protective immune responses against Nippostrongylus brasiliensis infection. The immune response generated during a primary infection by Nippostrongylus brasiliensis provides protection from re-infection. Long-term protection is dependent on CD4 ${ }^{+} \mathrm{T}$ cells but when sufficiently stimulated by cytokine, ILC2s can rescue the protection lost by the depletion of $\mathrm{CD} 4^{+} \mathrm{T}$ cells.

This thesis has shown that $\mathrm{CD} 4^{+} \mathrm{T}$ cells and populations of innate immune cells differentially regulate the expression of the closely related Type 2 cytokines IL- 4 and IL13. These discoveries will help direct future research aiming to boost the effectiveness of anti-helminth vaccines, or decrease the pathology caused by allergic diseases by targeting specific cytokine expression. 


\section{Acknowledgements}

I would never have been able to complete this thesis without the guidance, help and support of many people. Below I name those who had the greatest impacts but there are countless more whose passing comments and generosity have also shaped me, and this body of work, so I thank all who knowingly or not contributed to this thesis.

Thank you Graham Le Gros, my supervisor and mentor; through your guidance I have matured both personally and scientifically. I have learnt so much about the scientific process from your extensive experience and knowledge, I am proud to tack myself on to your scientific lineage. Thank you for supporting my growth and allowing me the space to explore and learn for myself. The Malaghan Institute of Medical Research is an amazing centre of research and training, it is in no small part due to your hard work that the institute is such an excellent place to work and learn. Thank you for taking me into your group and allowing me to experience what working in a top class facility with international collaborations is like. It has been a privilege working for you.

Elizabeth Forbes-Blom I aspire to know the literature of my field as comprehensively as you. Thank you for the time you have spent helping and talking to me about Type 2 immunity and the state and direction of New Zealand science and life in general.

Tiffany Bouchery and Ben Roediger, I have learnt so much from working with the both of you and thanks to you I have co-authored a number of high-class publications. Ben you have shown me how much can be gained through international collaboration, thank you for including me in your interesting and exciting research and being so supportive and generous. Tiffany, I cannot express how grateful I am that working with 
you was so uncomplicated, I enjoyed so much working together on the hookworm project, thank you for involving me in such a well-rounded study.

Mali Camberis thank you for helping me with all things Nippostrongylus brasiliensis and Heligmosomoides polygyrus, from worm culture to infection and counting I've gained form your expertise. You were always quick to assist with harvesting and by far the fastest tissue processor I know (I assure you this is a high honour). Mel Prout thank you for helping me whenever I needed it, I regret not beginning working with you earlier, your eye for detail and dealing with large amounts of data make working with you a treat. Shiau-Choot Tang thanks for helping me with the mysteries of PCR and genotyping. Past PhD students from our group Marcus Robinson and Helen Mearns, I owe you a lot, without your recommendations I may not have ended up in this group doing this work so thank you both for that. I appreciate your patience while training me and teaching me the basics of the Type 2 immune response. Thank you for setting solid models that foundations and I could build my work from.

The PhD student community at the Malaghan Institute is very supportive I particularly want to thank Alanna Cameron, Cameron Field, Catherine Plunkett, Sotaro Ochiai and Naomi Daniels. Our group of students have been great, from helping with experimental procedures, teaching each other techniques, delivering meals during late night experiments and providing moral support, I'm lucky to be surrounded by a great group of peers. I also want to thank my excellent research assistants. Arie BatesHermans and Giovanna Le Gros, the diligence and care you took with your work made my life much easier and freed me up to do more than I could have achieved my self.

Alfonso Schmidt, Bill Telford and particularly Kylie Price thank you for all your flow cytometry related support and advice. From helping with long days of sorting to assisting with late night and weekend machine break downs, the flow cytometry support at the Malaghan Institute is first class. Kylie and Bill thank you for developing the flow cytometers and working to find the optimal laser and filter configurations to detect AmCyan and make the reporter mouse a viable model. Thank you to the BRU staff for breeding and raising the mice, and dealing with my difficult cross breeding requests.

Thank you to Bill Paul (NAID, NIH, USA) for the reporter mice and for having me to visit the NIH. Thank you to Wolfgang Weninger (Centenary Institute, Sydney, Australia) for allowing Ben to work with me and for giving advice on my science. Thanks to Abbvie for generously providing the anti-IL-13 antibodies that allowed us to confirm our hypothesis. 
The Rex and Betty Coker scholarship has supported me throughout my $\mathrm{PhD}$, I appreciate the generous donations from the Coker family that established the scholarship and allowed me to survive. I have received funding from the Wellington Medical Research Foundation, the J. L. Stewart postgraduate scholarship and the New Zealand branch of the Australian Society for Immunology for travel to conferences and laboratories to present my work, thank you to these bodies for providing money for these amazing experiences. The Health Research Council has provided funding for the work carried out in this thesis.

My family have been very supportive throughout my education. Thank you to my grandparents, uncles and aunties who always show interest in what I am doing, and Leah for being a special friend. Gemma I hope you learn from my experiences and take them into account while making your own. Thank you Mum and Dad for helping me in so many ways. Without you I could not have achieved so much and I hope that by making you proud I can begin paying you back everything you have given me.

Finally I want to express my heartfelt appreciation to my long time friend and recent wife. Thank you Raima for your support and love, knowing that you will always greet me home with joy and enthusiasm helps me get through the toughest of days. Thank you for hearing out my gripes and celebrating my successes. You bring me so much happiness. I love you. I can't promise I'll have any more free time but at least (hopefully) you can finally claim you married a doctor. 


\section{Disclosure statement}

Many of the experiments carried out during my $\mathrm{PhD}$ have been in the context of collaborative efforts, both within the Asthma, Allergies and Parasitic Diseases group and with international collaborators. Initial Th2 culture experiments were carried out under the technical guidance of Marcus Robinson and Helen Mearns, both of who originally conceived of using the huCD2/GFP reporter mice to track allelic expression of IL4. Melanie Prout assisted with intradermal injections of HDM and with harvest, tissue processing and antibody staining of pTh2 samples. Arie Bates-Hermans, Amy Shepherd, Alanna Cameron, Catherine Plunkett and Mali Camberis have helped with tissue processing during large experiments. Ben Roediger and Wolfgang Weninger discovered the dermal innate lymphoid cell population at the Centenary Institute in Sydney, Ben Roediger and I had significant discussions about the models which I should use to look at the function of these cells.

Mali Camberis, Tiffany Bouchery and I worked together researching the mechanisms that mediate protection against Nippostrongylus brasiliensis (chapter 6). Tiffany and myself designed the experiments, Mali and Tiffany carried out many of the intravenous infections. During large harvests, Mali would often count larvae while Tiffany and I prepared single cell suspensions from tissues. All flow cytometry data presented in this thesis was collected and analysed myself, and samples were stained with antibody panels I designed.

With these disclosures, I declare that the content of this document is my own work. 


\section{Table of Contents}

Abstract

Acknowledgements ...................................................................ii

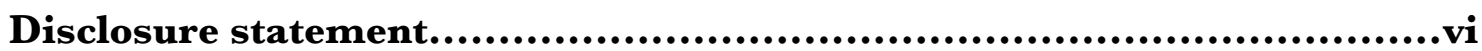

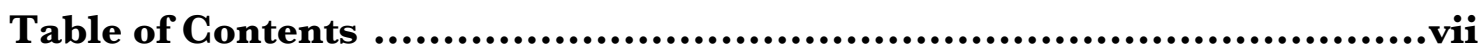

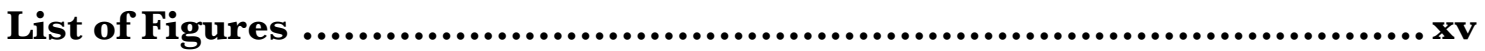

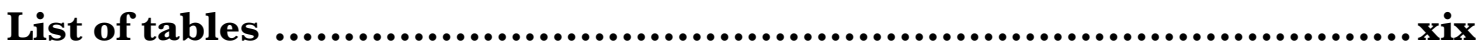

Abbreviations ............................................................................... xxi

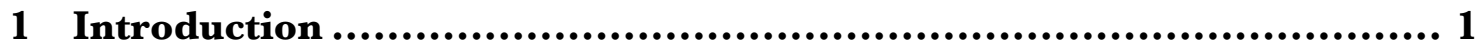

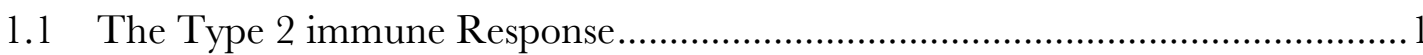

1.2 The activation, differentiation and physiological functions of $\mathrm{CD}^{+} \mathrm{T}$ cells........ 3

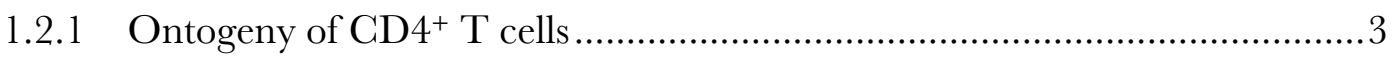

1.2.2 Activation of naïve $\mathrm{CD}^{+} \mathrm{T}$ cells .............................................................

1.2.3 Differentiation of activated $\mathrm{CD}^{+} \mathrm{T}$ cells .................................................

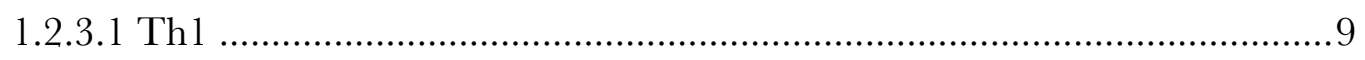

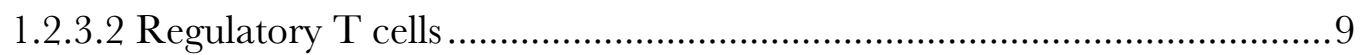

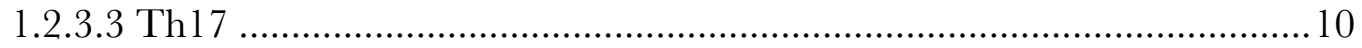

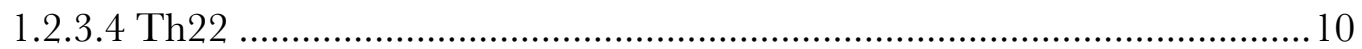




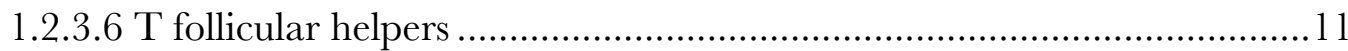

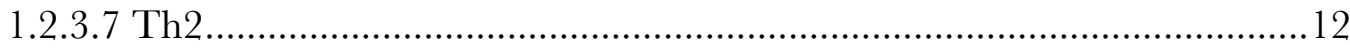

1.2.4 Mechanisms influencing $\mathrm{CD}^{+}{ }^{+} \mathrm{T}$ cell differentiation ...............................12

1.2.5 The contribution of dendritic cells to differentiation ...............................12

1.2.6 The quantity and quality of $\mathrm{T}$ cell receptor stimulation influences

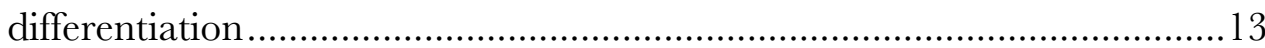

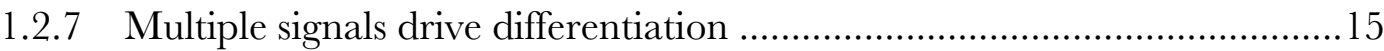

1.3 T helper 2 cells; orchestrators of Type 2 immunity .......................................17

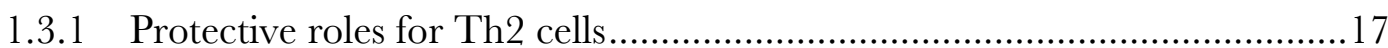

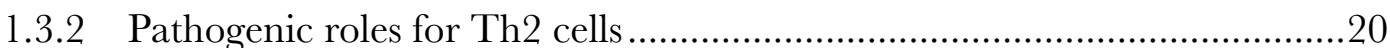

1.3.3 Immunopathologies as side effects of protective Type 2 responses against helminths .22

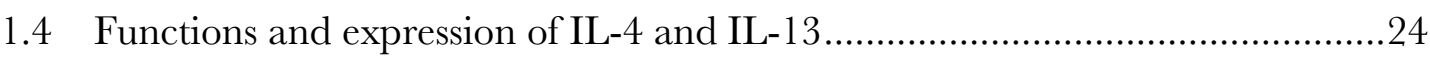

1.4.1 IL-4 and IL-13 receptor complexes ...................................................26

1.4.2 Lymphocyte responses to IL-4 and IL-13 ..........................................27

1.4.3 Alternate activation of macrophages ….............................................28

1.4.4 Responses by non-haematopoietic cells to IL-4 and IL-13 ........................30

1.4.5 Intracellular controls on the expression of IL-4 and IL-13 _......................33

1.4.6 Probabilistic regulation of IL-4 expression ................................................34

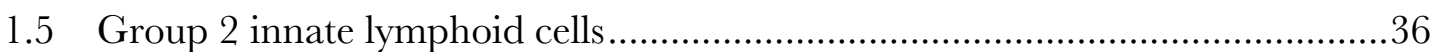

1.5.1 The populations of innate lymphoid cells ................................................36

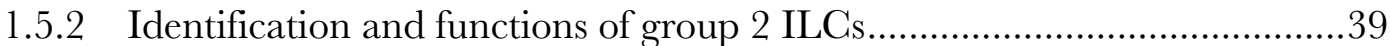

1.5.3 Reporter mice for identification of cytokine producing cells; the benefits and caveats .43

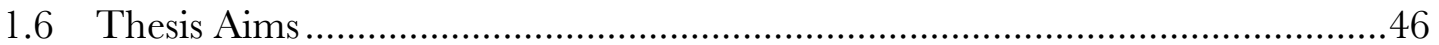

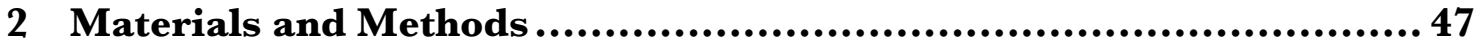

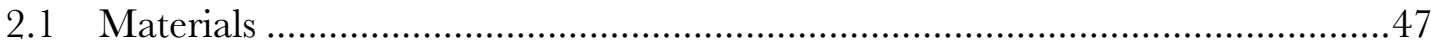




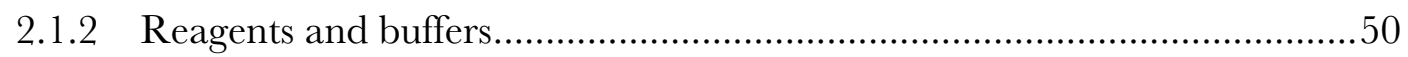

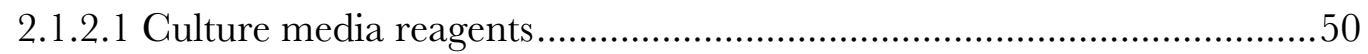

2.1.2.2 Enzymes and tissue processing reagents ...........................................50

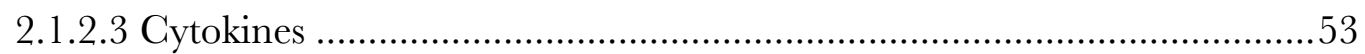

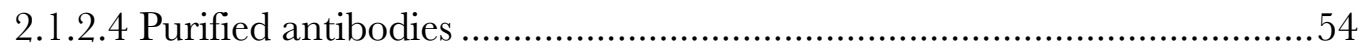

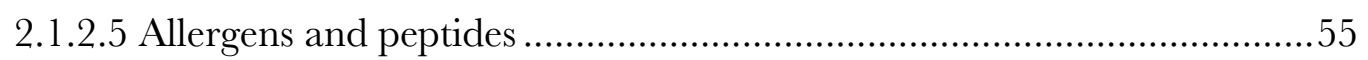

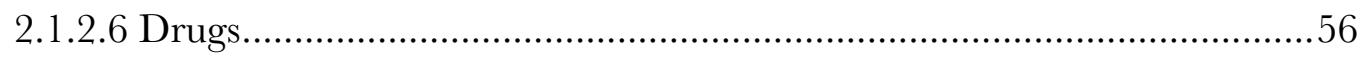

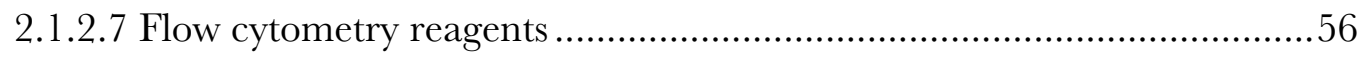

2.1.2.8 Fluorescently conjugated antibodies ..................................................58

2.1.2.9 Polymerase chain reaction reagents.................................................60

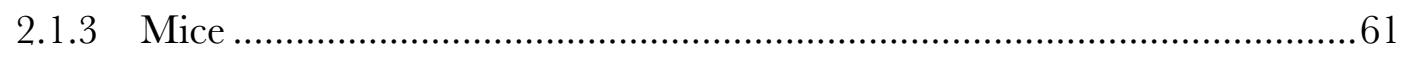

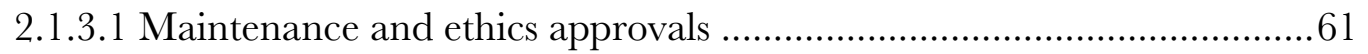

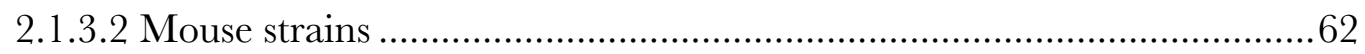

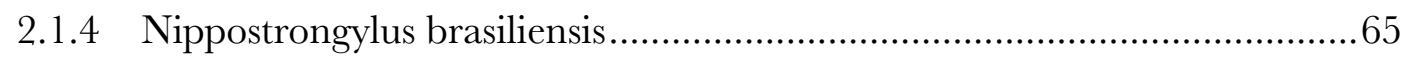

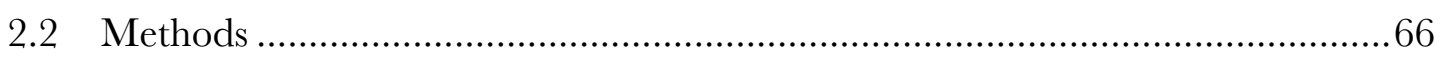

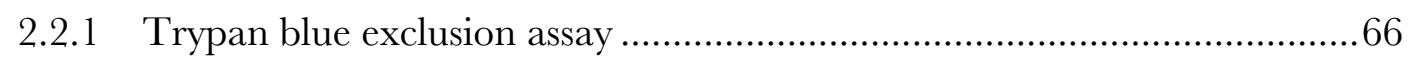

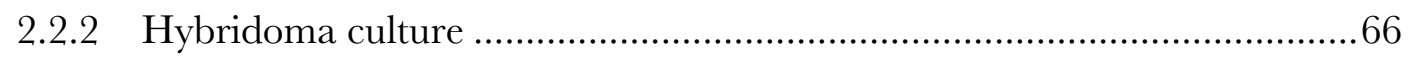

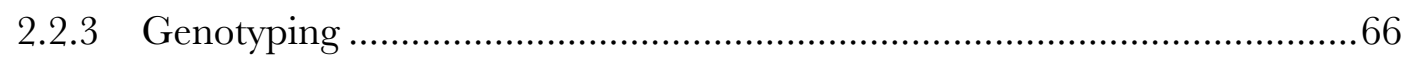

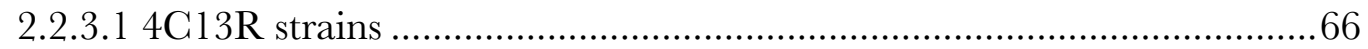

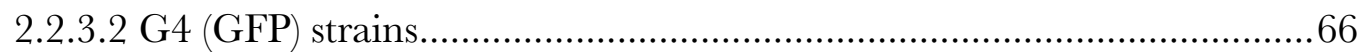

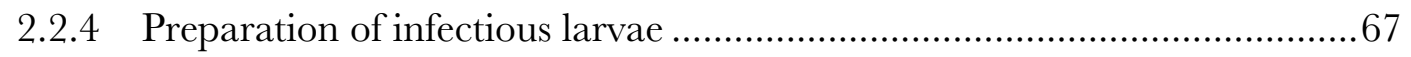

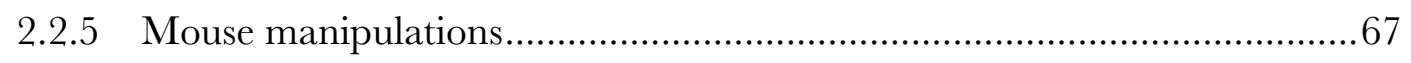

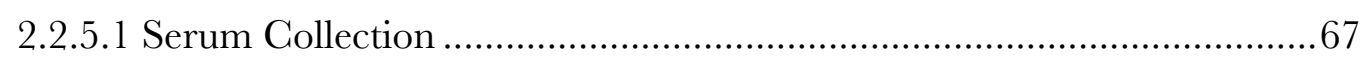

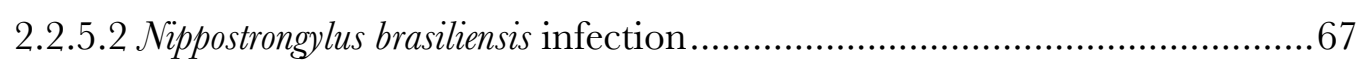

2.2.5.3 Primary Th2 immune response assay (pTh2 assay)...........................67

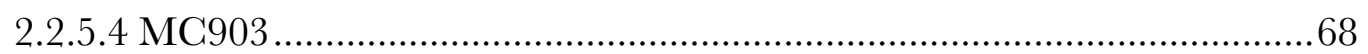




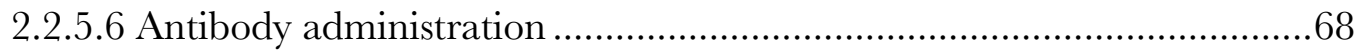

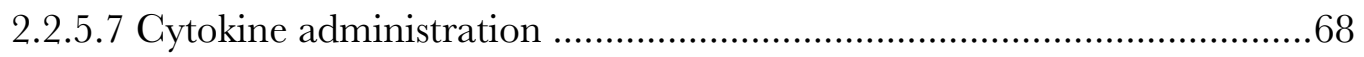

2.2.6 Tissue processing for larval counts....................................................69

2.2.7 Tissue processing for single cell suspension ........................................69

2.2.7.1 Spleen for flow cytometry and Th polarising cultures .........................69

2.2.7.2 Spleen for antigen presenting cells .................................................69

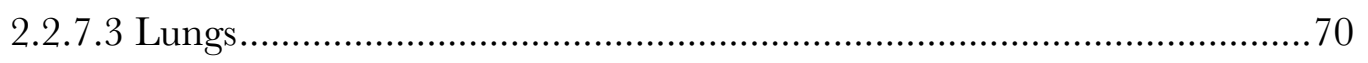

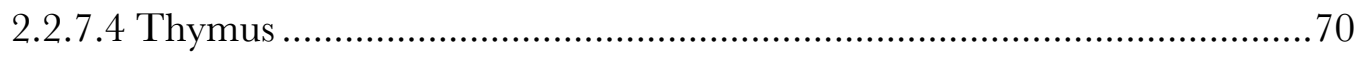

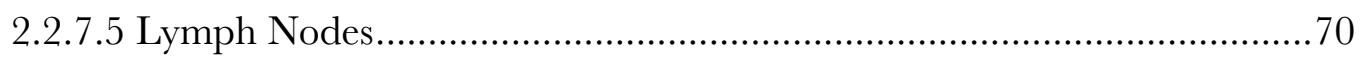

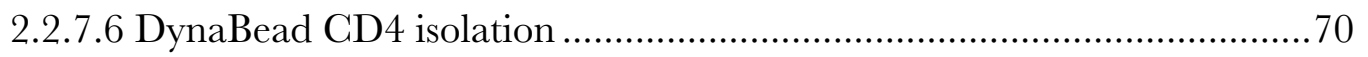

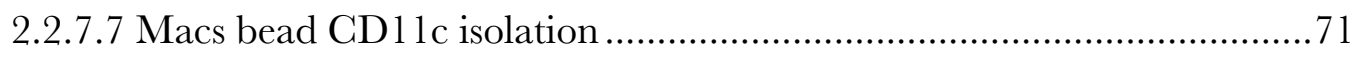

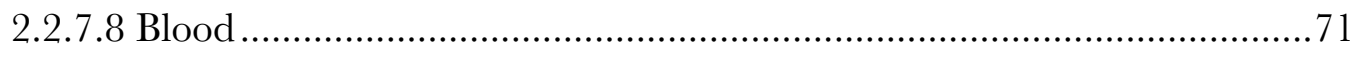

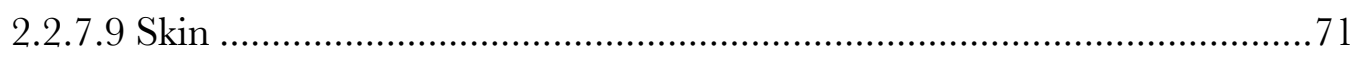

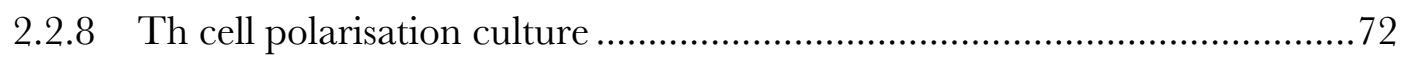

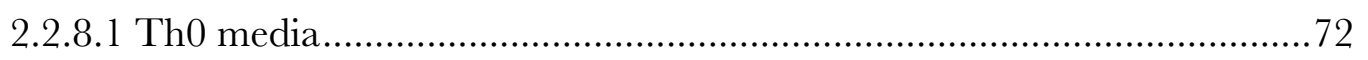

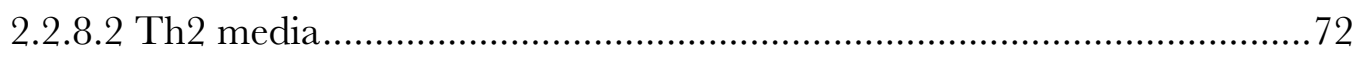

2.2.8.3 Carboxyfluorescein succinimidyl ester (CFSE) staining.........................73

2.2.8.4 Antigen processing cell - OTII cell culture ........................................73

2.2.9 Enzyme-linked immunosorbent assay (ELISA) ….................................73

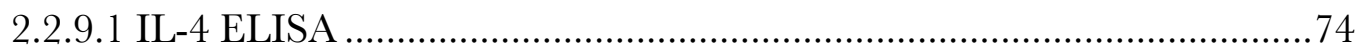

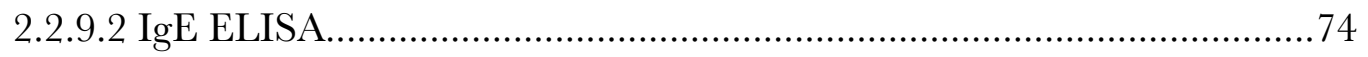

2.2.10 Flow cytometry staining and analysis................................................74

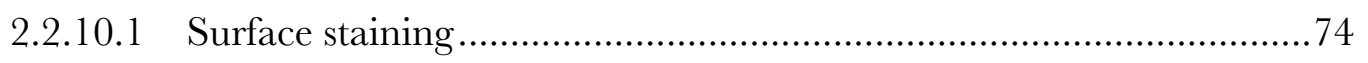

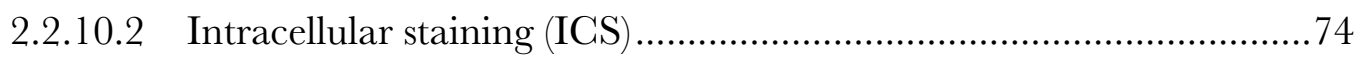

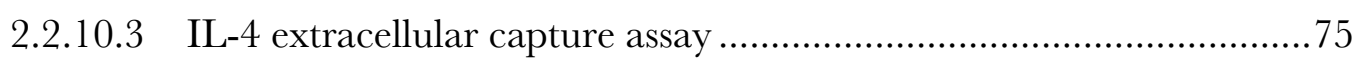

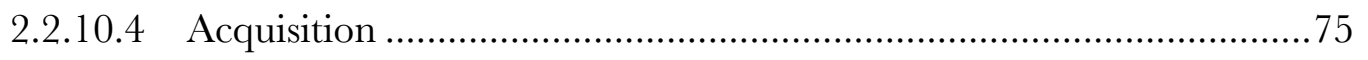




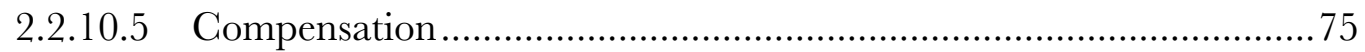

2.2.10.6 Fluorescence activated cell sorting.................................................. 76

2.2.10.7 Analysis of flow cytometry data ….................................................

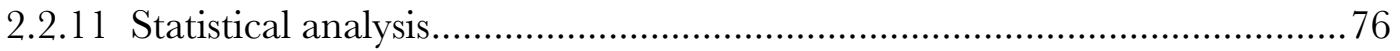

3 Mechanisms regulating expression of IL-4 by $\mathrm{CD4}^{+} \mathrm{T}$ cells .............. 77

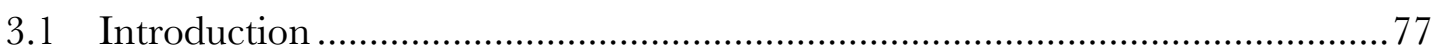

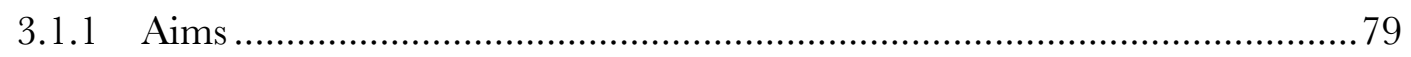

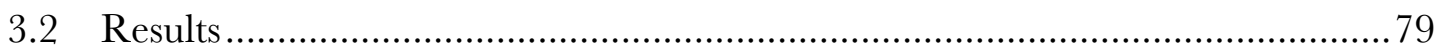

3.2.1 Analysis of IL-4 and IL-13 expression validates the 4C13R reporter mice 79

3.2.2 The different IL-4 reporter mice can be independently used to identify

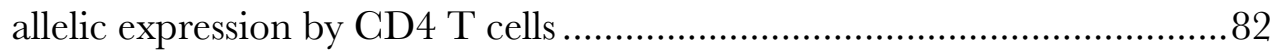

3.2.3 T cell receptor stimulation affects IL-4 allelic expression profiles by CD4 ${ }^{+}$ T cells. .86

3.2.4 Allelic IL-4 expression patterns are not maintained by restimulated CD4 ${ }^{+}$ $\mathrm{T}$ cells

3.2.5 Expression of the 4C13R transgenic IL-4 reporter is allelically regulated .95

3.2.6 Triple allele IL-4 reporter system indicates the special nature of biallelically expressing $\mathrm{CD} 4^{+} \mathrm{T}$ cells.

3.2.7 IL-4 is not important for the expansion of Th2 cells in vivo ......................100

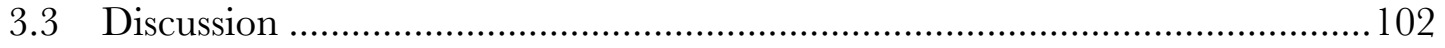

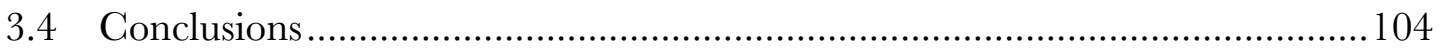

4 IL-13 production by $\mathrm{CD4}^{+} \mathrm{T}$ cells is regulated separately to IL-4..... 107

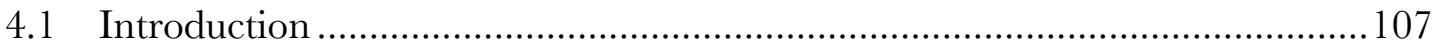

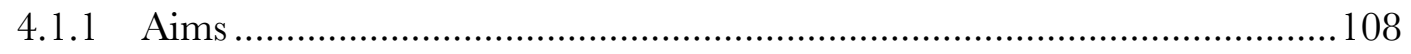

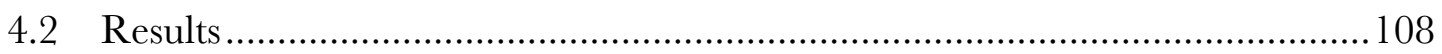

4.2.1 Th2 differentiated $\mathrm{CD}^{+}{ }^{+} \mathrm{T}$ cells begin expressing IL-4 and IL-13 at different times in vitro. 108 
4.2.2 Sustained or multiple TCR engagements are required for IL-13 expression by $\mathrm{CD} 4^{+} \mathrm{T}$ cells

4.2.3 Tissue specific CD4 ${ }^{+} \mathrm{T}$ cell cytokine expression patterns are not observed with in vitro stimulation

4.2.4 FTY720 blockade of LN egress affects both IL-4 and IL-13 expression by CD4 ${ }^{+} \mathrm{T}$ cells .130

4.3 Discussion .132

4.4 Conclusions .133

5 Innate immune cells of the skin display divergent expression of Type 2 cytokines 135

5.1 Introduction .135

5.1.1 Aims 136

5.2 Results 136

5.2.1 Dermal innate lymphoid cells are a distinct population of skin resident immune cells 136

5.2.2 IL-13 is constitutively expressed by dermal innate lymphoid cells ..... 141

5.2.3 dILCs only display minor responses in models of skin inflammation..... 144

5.2.4 Basophils respond to TSLP induced by MC903 treatment by producing IL-4 .152

5.3 Discussion. 159

5.4 Conclusions 162

6 Interplay of adaptive and innate immune responses mediate protection against Nippostrongylus brasiliensis ............................... 163

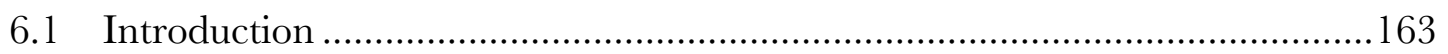

6.1.1 Aims 165

6.2 Results.... 165

6.2.1 ILC2s contribute to IL-13 production in the lung during $\mathcal{N}$. brasiliensis infection. 165 
6.2.2 $\mathrm{CD}^{+} \mathrm{T}$ cells support ILC2 responses in secondary $\mathcal{N}$. brasiliensis infection

6.2.3 ILC2 stimulating cytokines can induce protective immune responses in naïve mice

6.3 Discussion 186

6.4 Conclusions 189

7 General Discussion 191

7.1.1 The allelic expression of IL-4 is controlled by multiple factors 191

7.1.2 Transgenic reporter constructs are under similar regulation as parental alleles

7.1.3 Th2 IL-4 expression is independent of IL-4 signalling in vivo 195

7.1.4 The expression of IL-4 and IL-13 is differentially regulated 196

7.1.5 Innate immune cells associated with Type 2 display differential cytokine regulation

7.1.6 CD4+ T cells support ILC2 responses ...............................................202

7.1.7 CD4 ${ }^{+} \mathrm{T}$ cells are the central mediator of Type 2 immunity ...................2204

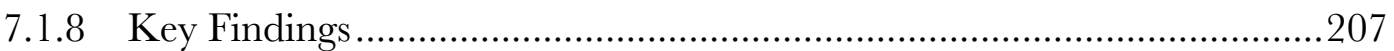

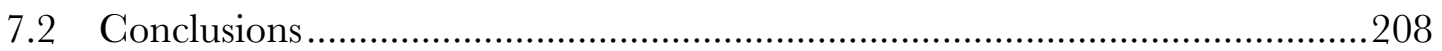

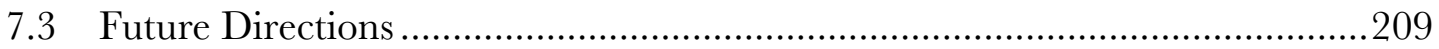

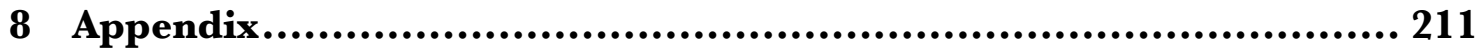

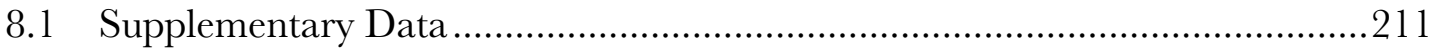

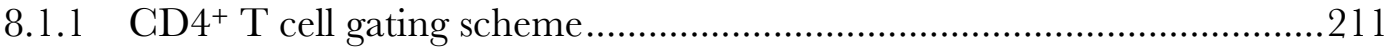

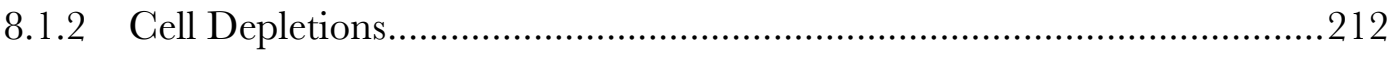

8.1.2.1 Depletion of CD4 ${ }^{+} \mathrm{T}$ cells from the lung .......................................2212

8.1.2.2 Depletion of ILC2s from the lung..................................................213

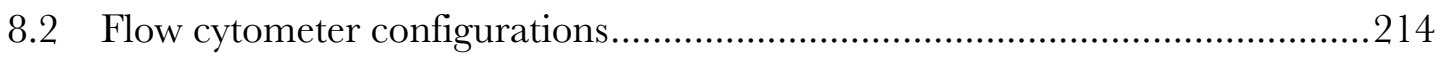

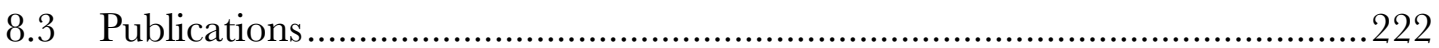

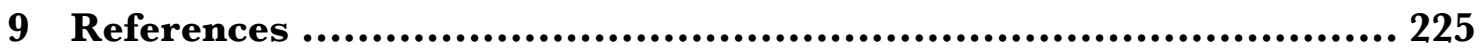




\section{List of Figures}

Figure 1.1 - The differentiation phenotypes of activated $\mathrm{CD} 4^{+} \mathrm{T}$ cells

Figure $1.2-\mathrm{CD}^{+} \mathrm{T}$ cells and IL-4/IL-13 signalling are central to Type 2 immune responses. 25

Figure 1.3 - Ontogeny of the innate lymphoid cell populations. 37

Figure 3.1 - 4C13R mice demonstrate only slight deficiencies in Type 2 cytokine production.

Figure 3.2 - 4C13R IL-4 reporting displays similar kinetics to knock-in reporter mice in vitro. .84

Figure 3.3 - IL-4 expression is under allelic control. .86

Figure 3.4 - IL-4 allelic expression patterns are unaffected by feeding regime. .88

Figure 3.5 - Anti-CD3 concentration affects reporter expression and bi-allelism. .90

Figure 3.6 - huCD2 can be transferred between cells in culture. .92

Figure 3.7 - Sorted populations do not retain their IL-4 allelic patterns upon restimulation.

Figure 3.8 - Expression of the 4G13R transgenic IL-4 reporter is under allelic control. . 97

Figure 3.9 - Qualities of multi-allelically IL-4 expressing cells elucidated by use of triple reporter mice.

Figure 3.10 - IL-4 is not required for the expansion of IL-4 producing CD4+ ${ }^{+}$cells.

Figure 4.1 - AmCyan and DS-Red are expressed at different times in vitro. 110 
Figure 4.2 - Neither feeding with Th2 culture cytokines, adding IL-33 nor enriching for CD4 induces earlier IL-13 induction.

Figure 4.3 - Previously activated/memory cells express DS-Red earlier in vitro than naïve cells.

Figure 4.4 - Sustained TCR stimulation is required for IL-13 expression. .117

Figure 4.5 - APG presentation does not induce IL-13 expression by CD4+ T cell. .....119

Figure 4.6 - Addition of extra peptide loaded APGs induces $\mathrm{CD}^{+} \mathrm{T}$ cell IL-13 expression in a TCR dependent manner

Figure 4.7 - Lung CD4+ ${ }^{+} \mathrm{T}$ cells express both AmCyan and DS-Red in response to $\mathcal{N}$. brasiliensis infection.

Figure 4.8 - LN CD4+ T cells do not express DS-Red.

Figure 4.9 - In vitro Th2 differentiated $\mathrm{CD} 4^{+} \mathrm{T}$ cells from $\mathrm{LN}$ and lung display the same pattern of cytokine reporting.

Figure 4.10 - Secondary infection leads to increases in IL-13 reporting CD4 ${ }^{+} \mathrm{T}$ cells compared with primary infection.

Figure 4.11 - Inhibition of LN egress with FTY720 has differential effects on IL-4 and IL-13 during $\mathcal{N}$. brasiliensis infection. 131

Figure 5.1 - dILGs are a unique population of skin immune cells.............................. 138

Figure 5.2 - dILGs have a phenotype characteristic of ILGs from other tissues............ 140

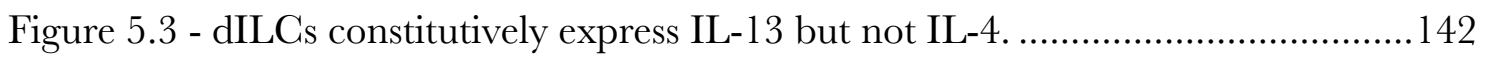

Figure 5.4 - IL-13 expression varies with age. 143

Figure 5.5 - Calcipotriol (MC903) causes inflammation and thickening of skin tissue. 145

Figure 5.6 - ILGs only show moderate responsiveness in a MC903 model of skin inflammation.

Figure 5.7 - dILCs show marginal responses to various inflammatory stimuli. 149

Figure 5.8 - ILCs of the skin are resistant to antibody depletion. .151

Figure 5.9 - Basophils migrate to the skin in response to MC903 treatment. 153

Figure 5.10 - Basophils migrate specifically to the site of treatment and the draining LN.

Figure 5.11 - MC903 elicited basophils produce IL-4 but not IL-13. 156

Figure 5.12 - TSLPR signalling is required for basophil IL-4 expression and migration to the $\mathrm{LN}$. .158

Figure $6.1-\mathcal{N}$. brasiliensis responsive DS-Red ${ }^{+}$non-CD4 lymphoid cells in the lung are ILC2s. 166 
Figure $6.2-\mathrm{CD}^{+} \mathrm{T}$ cells and ILC2s are the majority IL-13 producing cells during secondary infection.

Figure 6.3 - Requirement for IL-4 or IL-13 signalling to maintain protection.

Figure $6.4-$ ILC2s respond quickly to a primary $\mathcal{N}$. brasiliensis infection and match $\mathrm{T}$ cell IL-13 responses in a secondary infection. 173

Figure $6.5-\mathrm{CD} 4 \mathrm{~T}$ cells are required for long term protection but are not effector cells of the immune response. 175

Figure 6.6 - Secondary ILC2 responses are decreased in the absence of CD4 T cells. 177 Figure 6.7 - Lung ILC2s are responsive to IL-33, IL-2c and combinations of cytokine.

Figure 6.8 - IL-33 treatment can provide protection in the absence of CD4 T cells. ..181

Figure 6.9 - IL-2c activated ILC2s are required for protective responses.

Figure 6.10 - Treatment with IL-2c rescues protection in the absence of CD4 T cells. 185

Figure 7.1 - Summary of discoveries from this thesis in the greater context of Type 2 immune responses 206

Figure 8.1 - Panel for the identification of $\mathrm{CD} 4^{+} \mathrm{T}$ cells 211

Figure $8.2-\mathrm{CD}^{+} \mathrm{T}$ cell depletion in the lung is maintained for 4 days around infection.

Figure 8.3 - Panel for assessing lung ILC2 depletion with $\alpha \mathrm{CD} 90$ antibody. 213 


\section{List of tables}

Table 2-1 - List of fluorescently labelled antibodies used in this thesis for flow cytometry. 60

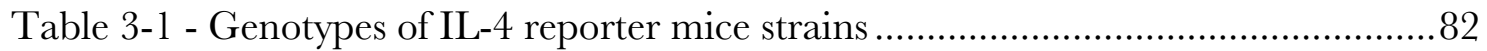

Table 8-1 - Laser and filter configurations of the BD LSR II SORP and BD LSR Fortessa flow cytometers. .221 


\section{Abbreviations}

$\begin{array}{ll}\text { AAM } & \text { Alternately activated macrophages } \\ \text { AHR } & \text { Airways hyperreactivity } \\ \text { Ahr } & \text { Aryl hydrocarbon receptor } \\ \alpha \text { (prefix) } & \text { Anti } \\ \text { AP-1 } & \text { Activator protein 1 } \\ \text { APC } & \text { Antigen presenting cells } \\ \text { BAC } & \text { Bacterial artificial chromosome } \\ \text { BALF } & \text { Bronchial-alveolar lavage fluid } \\ \text { CD } & \text { Cluster of differentiation } \\ \text { CFSE } & \text { Carboxyfluorescein succimidyl ester } \\ \text { CHILP } & \text { Common helper-like ILC progenitor } \\ \text { CILP } & \text { Common ILC progenitor } \\ \text { CLP } & \text { Common lymphoid progenitor } \\ \text { CTLA4 } & \text { Cytotoxic T-lymphocyte-associated protein } 4 \\ \text { DAG } & \text { Diacylglycerol } \\ \text { DC } & \text { Dendritic cell } \\ \text { dILC } & \text { dermal innate lymphoid cell } \\ \text { dLN } & \text { Draining LN } \\ \text { DNA } & \text { Deoxyribonucleic acid } \\ \text { ECM } & \text { Extracellular matrix } \\ \text { ELISA } & \text { Enzyme linked immunosorbant assay } \\ & \end{array}$


ER Endoplasmic reticulum

FACS Fluorescence-activated cell sorting

FCS Forward scatter

FoxP3 Forkhead box P3

GADS GRB2-related adaptor downstream of Shc

$\gamma \mathrm{c}$

GC

Common gamma chain

Gfil Growth factor independent 1

GFP Enhanced green fluorescent protein

GM-CSF Granulocyte-macrophage colony stimulating factor

HDM House dust mite

HS Hypersensitivity

huCD2 Inactive human CD2 used to identify IL-4-producing cells in the KN2 reporter

i.n. Intranasal

i.p. Intraperitoneal

i.v. Intravenous

ICOS Inducible T cell co-stimulator

ICS Intracellular cytokine staining

Ig Immunoglobulin

IкB Inhibitor of $\kappa \mathrm{B}$

IKK Inhibitor of $\kappa \mathrm{B}$ kinase

IL- $\quad$ Interleukin

ILG Innate lymphoid cell

$\mathrm{IP}_{3} \quad$ Inositol $1,4,5$ triphosphate

IRES Internal ribosome entry site

ITAM Immunoreceptor tyrosine-based activation motif

iTreg induced regulatory $\mathrm{T}$ cell

KLRG1 Killer cell lectin-like receptor subfamily G member 1

LAT Linker of $\mathrm{T}$ cells

LN Lymph node

LPS Lipopolysaccharide

Lti Lymphoid tissue inducer

xxii 
M2 Alternately activated macrophages

MAPK Mitogen-activated protein kinase

MFI Median fluorescent index

MHC II Major histocompatibility complex class II

MPP Multipotent progenitors

MR Mannose receptor

NFAT Nuclear factor of activated T cells

NK Natural killer

NKкB Nuclear factor $\kappa$ B

nTreg natural regulatory $\mathrm{T}$ cell

OVA Ovalbumin

$\mathrm{p}$ (prefix) Phosphorylated

PCR Polymerase chain reaction

PD-1 Programmed cell death protein-1

$\mathrm{PIP}_{2} \quad$ Phosphatidylinositol 3,4-bisphosphate

PKC- $\theta \quad$ Protein kinase C- $\theta$

PLC $\gamma \quad$ Phospholipase C- $\gamma$

PMA Phorbol 12-myristate 13-acetate

pTh2 Primary Th2 immune response assay

$\mathrm{R}$ (suffix) Receptor

RasGRP RAS guanine nucleotide releasing protein

RBC $\quad$ Red blood cell

Relmo Resistin-like molecule $\alpha$

Ror $\alpha \quad$ Retinoic acid receptor $\alpha$

ROR $\gamma \mathrm{t} \quad$ RAR orphan receptor $\gamma$

SSC Side scatter

STAT Signal transducer and activator of transcription

TGF-1 T cell factor 1

TCR T cell receptor

Tfh T follicular helper

TGF- $\beta \quad$ Transforming growth factor $\beta$

Th T helper

TNF- $\alpha \quad$ Tumour necrosis factor $\alpha$ 
TSLP Thymic stromal lymphopoietin

TTP Tristetraprolin

VCAM Vascular cell adhesion molecule-1

WT Wild type

xxiv 



\section{Introduction}

\subsection{The Type 2 immune Response}

The immune system is a network of cells found in all tissues of the body. These cells communicate by the production of molecules that they either express on their cell surface or release into their environment, these molecules interact with receptors on other cells, allowing coordination of actions and targeted responses against potential threats. By communicating and working together the cells of the immune system keep the body free from invading pathogens, control the commensal microbes that live on the body and maintain homeostasis of many body systems. To be effective the immune system must be able to mount different responses against different threats, an immune response against one type of pathogen such as a virus may not work against a multicellular parasite, so the immune system must have the ability to modify its approach on a case-by-case basis. Despite this flexibility there are common types of immune responses initiated against related groups of pathogens. The cells and signalling proteins that contribute to clearance of a bacterial infection are similar to the cells and signals that the immune system employs to clear intracellular prokaryote pathogens ${ }^{1}$. Type 1 responses are associated with the clearance of viruses and intracellular pathogens. Type 2 responses are related to the expulsion of multicellular parasites such as helminths, responses to ectoparasites such as ticks, and wound healing following infection. However, when activated against innocuous substances Type 2 responses can be pathogenic, causing diseases including allergies and asthma². 
CD4 ${ }^{+}$T cells expressing the $\alpha$ and $\beta$ T cell receptor chains, and differentiated towards a specific activation phenotype, named T helper 2 (Th2), are critical for orchestrating Type 2 immunity ${ }^{3}$. Through the production of specific signalling molecules called cytokines, Th2 $\mathrm{CD}^{+} \mathrm{T}$ cells mediate the recruitment, expansion and activation of a number of myeloid derived cells ${ }^{4,5}$. Th2 cells also mediate $\mathrm{B}$ cell activation and direct activated $\mathrm{B}$ cells to produce the $\operatorname{IgE}$ antibody isotype; increased serum $\mathrm{IgE}$ is strongly correlated with Type 2 immune responses ${ }^{6}$. The granulocytes, mast cells, basophils and eosinophils are also associated with Type 2 immune responses. These cells carry granules packed with enzymes, toxic products, histamine and cytokines that they release upon activation ${ }^{7}{ }^{8}$. Cross-linking of surface bound $\operatorname{IgE}$ molecules by an antigen is a signal that drives activation and degranulation of basophils and mast cells ${ }^{9}$. Granulocytes can also actively produce large amounts of Type 2 associated cytokines ${ }^{10}$. Macrophages respond to Type 2 cytokines by exhibiting an alternate activation phenotype. These macrophages are implicated in both protection against parasites and wound healing ${ }^{11}$. Type 2 immune responses can also affect non-immune cells, with cytokines produced by Th2 cells directly activating fibroblasts and increasing extracellular matrix deposition ${ }^{12}$. This can assist wound healing, but in cases of excessive Type 2 responses, may lead to fibrosis and loss of function in tissues including the lung and liver ${ }^{13}$. Increased smooth muscle function is also seen with Type 2 signalling, which can assist the expulsion of intestinal parasites ${ }^{14}$. A recently discovered subset of lymphoid cells, devoid of antigen specific receptors but able to produce Type 2 cytokines in responses to epithelial derived signals, have been labelled the group 2 innate lymphoid cells (ILCs) ${ }^{15}$. While these cells have been implicated in models of parasite expulsion and asthma, further investigation is required to assess their roles in other models associated with Type 2 inflammatory responses.

In this thesis I will characterise the expression of two cytokines produced by several cellular subsets that contribute to Type 2 immune responses, assessing the signals that induce and regulate the expression of these cell-signalling molecules in a range of in vitro and in vivo models. I will investigate the mechanisms via which $\mathrm{CD} 4^{+} \mathrm{T}$ cells control their production of the Type 2 cytokines interleukin-4 (IL-4) and interleukin-13 (IL-13) by utilising both newly generated and existing cytokine reporter mice. Using the same reporter systems the contributions of ILCs and basophils to inflammatory responses in the skin will be evaluated. Finally, I will examine how both $\mathrm{CD} 4^{+} \mathrm{T}$ cells and ILCs mediate protective responses in the lung against hookworm infection. Integrating the 
knowledge gained from this body of work will allow greater understanding of how individual cells regulate their cytokine responses, and how these cells work in the greater network that is the Type 2 immune response.

\subsection{The activation, differentiation and physiological functions of $\mathrm{CD}^{+} \mathrm{T}$ cells}

CD4 expressing T cells, known as T helper cells (Th cells) are important for mediating both innate and adaptive immune responses. These cells have roles in helping the production and isotype selection of antibodies by B cells, supporting and expanding $\mathrm{CD}^{+} \mathrm{T}$ cell responses and recruitment of cells from the innate arms of the immune system. CD4 ${ }^{+} \mathrm{T}$ cells are also very important in regulation and resolution of immune processes ${ }^{16}$. Due to their varied roles, $\mathrm{CD} 4^{+} \mathrm{T}$ cells are often likened to the generals of the immune cell army ${ }^{17}$. What allows $\mathrm{CD}^{+} \mathrm{T}$ cells to be so multifunctional is their ability to differentiate after activation into distinct subsets capable of producing specific signalling cytokines and driving specific immune responses ${ }^{18}$. This section will discuss the signals that drive activation and differentiation of the currently recognised $\mathrm{CD} 4^{+} \mathrm{T}$ cell subsets and the proposed roles of these cells.

\subsubsection{Ontogeny of CD4 ${ }^{+} \mathrm{T}$ cells}

$\mathrm{T}$ helper cells express the T cell receptor (TCR) $\alpha$ and $\beta$ chains, which along with the co-receptor molecule CD4, recognise peptides of 15-20 amino acids loaded in the cleft of the major histocompatibility complex class II (MHC II) molecule. All T cells derive from common lymphoid progenitors (CLPs), which migrate from the bone marrow to the cortex of the thymus ${ }^{19}$. While CLPs are double negative in respect to their CD4 and CD8 expression, they develop the expression of their TCR $\beta$ chain through germ line recombination. This is followed by the expression of CD8 and CD4, and finally by the generation and expression of the TCR $\alpha$ chain. Double positive $\left(\mathrm{CD} 4^{+} \mathrm{CD}^{+}\right)$cells with complete TCRs then begin the first of two rounds of selection. Positive selection requires appropriate interactions with MHC molecules, interactions that are neither too weak nor too strong lead to the cells receiving pro-survival signals. Cells that have sustained interactions with MHC class II molecules will down regulate expression of CD8 becoming $\mathrm{CD}^{+} \mathrm{T}$ cells. The $\mathrm{CD} 4^{+} \mathrm{T}$ cells then migrate to the thymic medulla and undergo negative selection, ensuring that auto-reactive cells are either forced into 
an apoptotic directive, or in some situations becoming the immune modulating natural $\mathrm{CD}^{+}$regulatory $\mathrm{T}$ cells (nTregs). ${ }^{20}$ The relatively few $\mathrm{CD} 4^{+} \mathrm{T}$ cells that successfully navigate these processes may then depart the thymus and begin circulating through the peripheral secondary lymphoid tissues sampling MHC II molecules, seeking the cognate peptide their TCR is specific for.

Unlike the nearly ubiquitous expression of MHC class I molecules, MHC II expression is restricted, with highest constitutive levels found upon professional antigen presenting cells (APGs) ${ }^{19}$. Professional APGs include dendritic cells (DCs), macrophages and B cells. During the synthesis of MHC II, the invariant chain blocks the occupation of the peptide-binding cleft by endogenous peptides. The MHC II molecules are assembled and trafficked from the endoplasmic reticulum, to the golgi body and eventually into vesicles where they are bound to the internal membranes. APGs, particularly DGs and macrophages, are adept at sampling environmental proteins, phagocytosing them through receptor mediated or random endocytosis, and packaging the proteins into endosomes. These endosomes, containing partially digested peptides, bind to vesicles where the invariant chains are sequentially removed from the MHC II binding cleft, allowing insertion of environmental peptides into the cleft. The endosomes with peptide carrying MHC II molecules are then transported to the cell surface where they merge with the cells external membrane. This results in surface presentation of the MHC II molecules and allows interactions with $\mathrm{CD} 4{ }^{+} \mathrm{T}$ cells. ${ }^{21}$

\subsubsection{Activation of naïve $\mathrm{CD}^{+} \mathrm{T}$ cells}

Once the naïve $\mathrm{CD}^{+} \mathrm{T}$ cells enter lymphatic circulation they begin interrogating MHC II expressing on APGs until they encounter a bound peptide that their TCR can bind with high enough affinity to induce signalling. The TCR chains cannot be expressed on the cell surface without associating with the CD3 complex ${ }^{22}$. The CD3 $\varepsilon$, CD38, CD3 $\gamma$ and CD3 $\zeta$ chains carry immunoreceptor tyrosine-based activation motifs (ITAMs), which are critical for downstream signalling of the TCR ${ }^{19}$. Once a TCR binds to a peptide-MHC II complex with sufficient affinity CD4 brings its associated intracellular tyrosine kinase Lck into close connection with the intracellular domains of the GD3 chains, allowing Lck to phosphorylate the CD3 chain ITAMs. This process is also assisted by another tyrosine kinase Fyn. Once phosphorylated the GD3 $\zeta$ chains can be bound by another tyrosine kinase Zap70 ( $\zeta$ chain associated protein 70$)$, which then 
phosphorylates the two scaffold proteins SLP-76 and linker of T cells (LAT) allowing binding with GRB2-related adaptor downstream of Shc (GADS) and phospholipase C- $\gamma$ (PLC- $\gamma$ ). Itk, itself having been activated by Lck, activates this complex after receiving a final phosphorylation. The activated PLC- $\gamma$ complex can cleave the membrane bound phosphatidylinositol 3,4-bisphosphate $\left(\mathrm{PIP}_{2}\right)$ and, along with phosphatidylinositol-3 $\left(\mathrm{PI}_{3}\right)$ kinase, generates inositol 1,4,5 triphosphate $\left(\mathrm{IP}_{3}\right)$ and diacylglycerol (DAG). These two molecules are instigators of three main transcriptional pathways downstream of the TCR, causing nuclear translocation of the transcription factors nuclear factor $\mathrm{\kappa} B$ (NFkB), nuclear factor of activated T cells (NFAT) and activator protein 1 (AP-1). ${ }^{19}, 23$, 24

$\mathrm{IP}_{3}$ release causes increased intracellular $\mathrm{Ca}^{2+}$ concentrations initially by opening calcium channels in the membrane of the endoplasmic reticulum (ER). The depletion of ER $\mathrm{Ca}^{2+}$ causes the opening of plasma membrane calcium channels leading to a massive flux of calcium into the cytosol. NFAT resides in the cytosol of cells, with its nuclear translocation blocked by phosphorylation. Calmodulin, activated in the presence of increased cytosolic $\mathrm{Ca}^{2+}$ concentrations, binds calcineurin and together these enzymes dephosphorylate NFAT. Dephosphorylated NFAT is then released from the cytosol and able to carry out its transcription factor functions. ${ }^{24}$

The mitogen-activated protein kinase (MAPK) pathway is an internal signalling cascade pathway common to many different receptors. In TCR activation DAG binds RAS guanine nucleotide releasing protein (RasGRP), which activates Ras. Ras is a small Gprotein that activates the first of the triad of MAP kinases, Raf that activates Mek, which subsequently activates Erk to phosphorylate the transcription factor Elk-1 leading to the transcription of the fos gene. In the nucleus c-FOS and phosphorylated c-Jun form the heterodimeric transcription factor AP- $1 .{ }^{25}$

DAG and increased intracellular $\mathrm{Ca}^{2+}$ concentrations together lead to the activation of protein kinase $\mathrm{C}-\boldsymbol{\theta}(\mathrm{PKC}-\boldsymbol{\theta})$, which recruits several proteins into a membrane-bound complex that binds and activates inhibitor of $\kappa \mathrm{B}$ kinase (IKK) complex. Once active IKK can, via ubiquitination and degradation, remove the inhibitor of $\kappa \mathrm{B}(\mathrm{I} \kappa \mathrm{B})$ from the NFKB molecule allowing it to enter the nucleus. The activation and translocation of these three transcription factors leads to the transcription of many genes, including $I l 2$ the importance of which will be discussed later in this section. ${ }^{25}$ 
Antigen recognition by the TCR is only the first of three signals required to fully activate $\mathrm{CD}^{+}{ }^{+} \mathrm{T}$ cells; without adequate co-stimulation TCR activated cells will become anergic and unresponsive to activation. Binding of the T cell expressed CD28 to CD80 and CD86 (also known as B7.1 and B7.2 respectively) on the APC is the primary and most important co-stimulatory signal required for sustained T cell activation. Binding of CD28 to the B7 complex leads to several signalling pathways that support and complement TCR activation. Binding of tyrosine kinases Lck and Itk to CD28 removes inhibition and boosts their kinase activity. CD28 can also recruit adaptor proteins that can activate the MAPK pathway, and it activates $\mathrm{PI}_{3}$ kinase that recruits Akt and has roles in inhibiting cell death, such as increasing BCL-X $\mathrm{X}_{\mathrm{L}}$ protein and inhibiting FAS signalling, thus increasing survival of the activated cell. CD28 signalling also increases activation of IKK, further removing inhibition of NFKB nuclear function. ${ }^{26}$ CD28 signalling contributes to the boosting of IL-2 secretion via several diverse mechanisms. The IL-2 promoter has binding sites for NFAT, NFkB, AP-1 and Oct1, these binding sites are also regulatory elements inhibiting transcription until bound by transcription factors. Octl is constitutively expressed in lymphocytes but the CD28 signalling induction of the other transcription factors assists the TCR driven deregulation of $I l 2$ transcription. CD28 ligation also supports IL-2 expression via posttranscriptional mechanisms. Cells have various mechanisms of degrading mRNA, one of which is production of proteins targeting AU-rich elements within the 3' untranslated regions, which are common in cytokine mRNA. TCR activation leads to the production of one of these proteins tristetraprolin (TTP), the absence of which causes augmented IL-2 production, and CD28 signalling has been shown to inhibit TTP driven degradation of IL-2 mRNA through as yet unresolved mechanisms. Through both preand post-transcriptional mechanisms CD28 co-stimulation leads to a 50-100 fold increase in IL-2 production by activated T cells. ${ }^{19,26}$

Cytokine signalling provides the third signal that promotes $\mathrm{T}$ cell activation. IL-2 in an important cytokine for driving optimal $\mathrm{CD}^{+} \mathrm{T}$ cell activation. Along with IL-2 production, expression of IL-2 receptor $\alpha$ (CD25) is also initiated by TCR ligation. CD25 is a high affinity receptor and has been found to first bind IL-2 then bind IL2R $\beta$ $(\mathrm{CD} 122)$ and the common gamma chain $(\gamma \mathrm{c})$, which brings in the intracellular signalling motifs. CD122 and $\gamma_{c}$ can bind IL-2 and cause signalling alone, but CD25 increases the receptor affinity significantly and stabilises the complex. The TCR driven expression of 
IL-2 and CD25 leads to autocrine and paracrine signalling through the IL-2R inducing MAPK and $\mathrm{PI}_{3} \mathrm{~K}$ pathways to boost cell survival and proliferation, and STAT5, which mediates expression of cytokines such as IL-4 and also causes a second, and greater increase of CD25 expression. Optimal activation of $\mathrm{CD}^{+} \mathrm{T}$ cells requires the three signals of TCR binding, co-stimulation and cytokine signalling, however the quality and quantity of the all three signals can dictate the activation phenotype a CD4 ${ }^{+} \mathrm{T}$ cell acquires. ${ }^{27}$

\subsubsection{Differentiation of activated $\mathrm{CD}^{+} \mathrm{T}$ cells}

The seminal paper in 1986 by Mosmann and Coffman first documented that clones of activated $\mathrm{CD}^{+} \mathrm{T}$ cells could be segregated into two populations based upon their cytokine secretion profiles. These subsets were defined as Thl identified by the production of interferon- $\gamma($ IFN- $\gamma)$, and the IL-4 producing Th2 cells ${ }^{28}$. It was found that IL-12, produced by APGs and other innate immune cells responding to proinflammatory signals, drives newly activated $\mathrm{CD}^{+} \mathrm{T}$ cells to a $\mathrm{Th} 1$ phenotype $^{29}$. For in vitro differentiation IL-4 is critical for Th2 cell generation ${ }^{30}$, though it appears to not be required in vivo as Th2 cells can be identified in IL-4 deficient mice ${ }^{31}$. Two differentially expressed transcription factors, T-bet and GATA3 were found to be required for stable differentiation into Th1 and Th2 respectively ${ }^{32,33}$.

In the past two decades it has been recognised that CD4+ $\mathrm{T}$ cells can occupy more than just two activation states ${ }^{34}$. Suppressive regulatory $\mathrm{T}$ cells were identified as a unique phenotype of $\mathrm{CD}^{+} \mathrm{T}$ cells ${ }^{35}$. Th17 cells, predominantly expressing IL-17 were subsequently discovered ${ }^{36}$ followed by IL-9 producing Th9s ${ }^{37}$ and IL-22 (but not IL-17) producing Th2238. CD4 ${ }^{+} \mathrm{T}$ cells that support antibody production reside either within the lymph node (LN) T and B cell zone borders or in the reactive germinal centre and were named $\mathrm{T}$ follicular helper ( $\mathrm{Tfh}$ ) cells and germinal centre $\mathrm{Tfh}$ (GC-Tfh) respectively ${ }^{39}$. Recently a putative granulocyte-macrophage colony stimulating factor (GM-CSF) producing population has been discovered but the in vivo relevance of these cells needs to be confirmed ${ }^{40}$. Briefly described below are the characterised $\mathrm{CD} 4^{+} \mathrm{T}$ cell activation phenotypes and their proposed roles in infection and disease. 


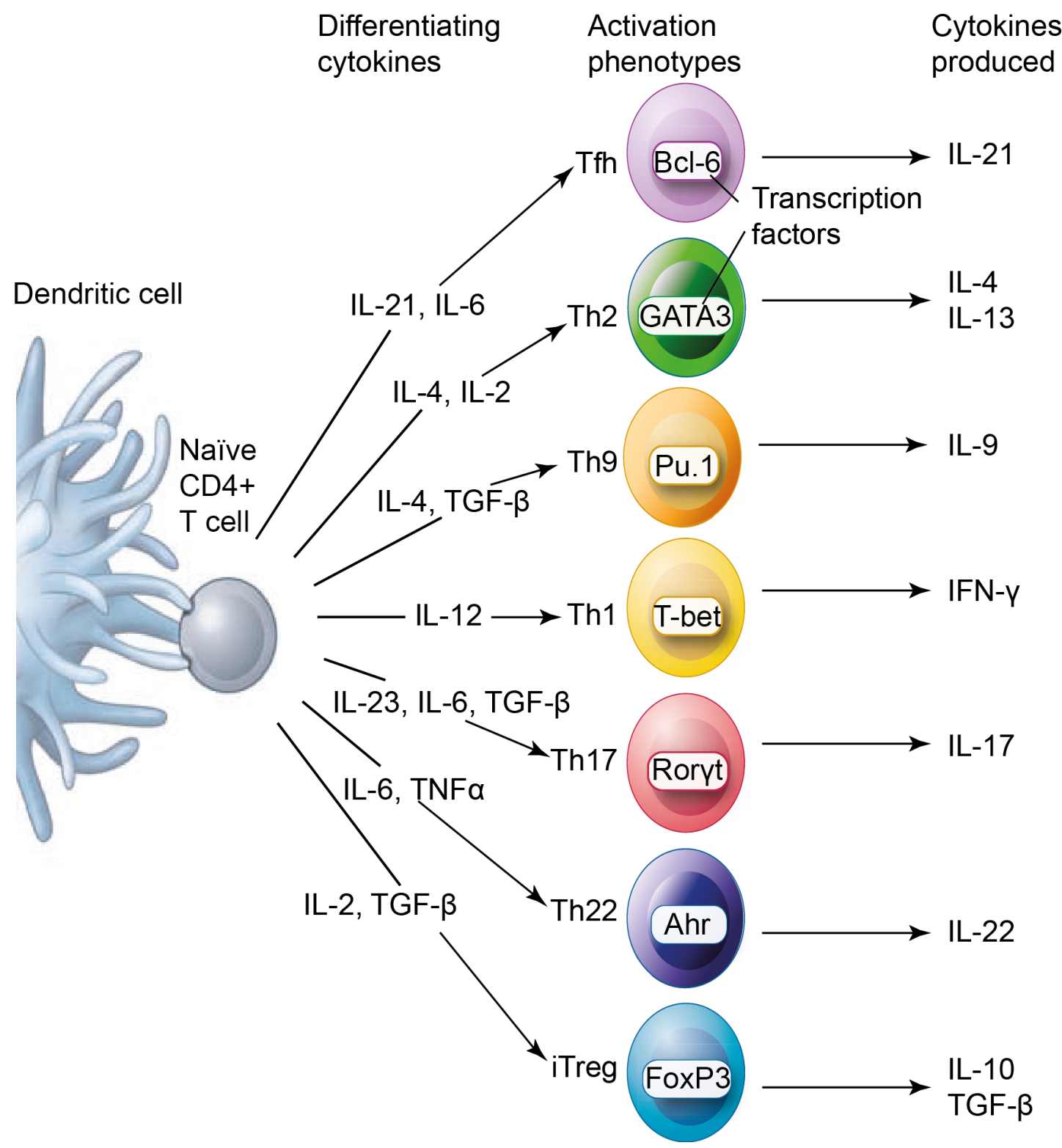

Figure 1.1 - The differentiation phenotypes of activated $\mathrm{CD4}^{+} \mathbf{T}$ cells.

Activated CD4+ $\mathrm{T}$ cells have been shown to differentiate into seven activation phenotypes that can be identified by their expression of specific transcription factors and cytokine profiles. The presence of some cytokines preferentially drive differentiation of certain phenotypes. These cytokines have been listed as differentiating cytokines, but there are varied requirements for these cytokines in vivo. Adapted from O'Shea and Paul, $2010^{34}$. 


\subsubsection{Th1}

The drivers of the Thl phenotype and what roles these cells play in immune responses have been well researched ${ }^{41}$. Initially it was shown that IL-12 was required for Listeria monocytogenes driven Th1 activation ${ }^{29}$, and later it was shown that IFN- $\gamma$ also played an important role in sustaining the Thl phenotype ${ }^{42}$. The transcription factor T-bet is the master regulator of the Thl phenotype, controlling the expression of IFN- $\gamma$, while also suppressing many of the genes associated with Th2 and the other Th phenotypes ${ }^{32}$. Th1 cells are characterised by their production of high levels of IFN- $\gamma$, as well as tumour necrosis factor- $\alpha$ (TNF- $\alpha$ ) and IL- $2^{43}$. Functionally, Th1 cell have been associated with the control of intracellular bacterial infections, such as mycobacterial species ${ }^{44}$. Their production of IFN- $\gamma$ activates macrophages, the major reservoir of these infections, increasing their bactericidal abilities ${ }^{45}$, while TNF- $\alpha$ can induce macrophage death depriving the bacilli of intracellular refuge ${ }^{46}$. Th1 cells also support B cell activation and isotype switching, driving the production of IgG2a, which can be important in controlling extracellular bacterial infections ${ }^{47}$.

\subsubsection{Regulatory $\mathrm{T}$ cells}

Regulatory $\mathrm{T}$ cells (Tregs) represent another potential phenotype of $\mathrm{CD} 4^{+} \mathrm{T}$ cells that demonstrate particular proficiency in inhibiting autoimmune inflammation ${ }^{48}$. The expression of $\mathrm{CD} 25$ on $\mathrm{CD}^{+} \mathrm{T}$ cells from naïve animals has been found to mark a population of Tregs known as natural Tregs (nTregs) that develop from cells with moderately high self-recognition during negative selection in the thymus ${ }^{49}$. Forkhead box P3 (FoxP3) is the master transcription factor maintaining Treg function; it represses the transcription of IL-2, IL-4, IFN- $\gamma$ and promotes CD25 and cytotoxic T-lymphocyteassociated protein 4 (CTLA-4) expression ${ }^{50}$.

Peripheral naïve $\mathrm{CD} 4^{+} \mathrm{T}$ cells can be stimulated in certain conditions to become one of several populations of Tregs; these populations are gathered under the generic title of inducible Tregs (iTregs $)^{51}$. In vitro activation of naive $\mathrm{CD} 4^{+} \mathrm{T}$ cells in the presence of TGF- $\beta$ induces FoxP3 expression; the addition of IL-2 further solidifies the Treg phenotype ${ }^{52}$. Tregs can express IL-10 and TGF- $\beta$, though the ability to suppress inflammatory responses depends on different factors in different models ${ }^{53}$. CTLA-4 expression by Tregs can signal back to APGs via CD80 and CD8654, causing downregulation of co-stimulatory ligands and production of indoleamine 2,3-diogenase (IDO) 
by the APCs, which cause death of T cells in the vicinity by converting tryptophan to the $\mathrm{T}$ cell toxic kynurenine ${ }^{55}$. The production of IL-10 by Tregs has been shown to be critical for inhibiting the development of colitis $^{56}$. Through their expression of the high affinity IL-2R $\alpha$ subunit CD25 it is also proposed that Tregs can act as IL-2-sinks, taking up available IL-2 limiting the support that newly activated effector cells would usually receive via autocrine or paracrine IL-2 signalling ${ }^{57}$. Tregs represent an important CD4 ${ }^{+}$ $\mathrm{T}$ cell phenotype; these cells are critical for inhibiting self-reactive $\mathrm{T}$ cell responses, inducing tolerance to foreign proteins and limiting and resolving immune reactions.

\subsubsection{Th17}

The discovery of the IL-12 related cytokine IL-23 lead to the recognition of a separate phenotype of effector CD4 ${ }^{+} \mathrm{T}$ cells, the Th17 cell $^{58}$. These cells can be identified by the expression of IL-17 and the master transcription factor, a splice variant of retinoic acid receptor related orphan receptor $\gamma(\operatorname{ROR} \gamma)$, ROR $\gamma \mathrm{t}^{59}$. Similar to Tregs, Th17 cells respond to TGF- $\beta$ for in vitro differentiation but also require a second signal. This was originally found to be IL-6, though this can be replaced by IL-21, and IL-1 signalling can replace TGF- $\beta$, indicative of redundancy in their activation. It was also found that IL-23 actually reinforces the Th17 phenotype of already activated cells. ${ }^{60}$ Th17 cells are critical in promoting antifungal immunity ${ }^{61}$, but inappropriate Th17 activation has been implicated in autoimmune responses in multiple sclerosis ${ }^{6261}$. Along with IL-17, Th17 cells also produce IL-22, IL-6, IL-21 and TGF- $\beta^{63}$. ROR $\gamma$ t and FoxP3 negatively regulate the $\mathrm{CD}^{+} \mathrm{T}$ cell differentiation programmes of Tregs or Th17 respectively ${ }^{64}$.

\subsubsection{Th22}

The production of the cytokine IL-22, part of the IL-10 family of cytokines, was originally attributed to Th17 cells. However, identification of cells producing IL-22 independently of IL-17 lead to the discovery of Th22 cells ${ }^{65}$. These cells rely upon the aryl hydrocarbon receptor as a master transcription factor ${ }^{66}$. Epithelial cells, keratinocytes and some fibroblasts, are the only cells which express IL-22R; signalling by IL-22 on these cells drives the production of antimicrobial products ${ }^{67}$. IL-22 production from Th22 cells is associated with controlling microbial colonisation of mucosal surfaces. Limiting the ability of potentially pathogenic microbes to colonise intestinal surfaces has been shown to be vital for controlling inflammatory colitis ${ }^{68}$ and mediating wound repair ${ }^{69}$. 


\subsubsection{Th9}

IL-4 and TGF- $\beta$ are required for optimal differentiation of a CD4 ${ }^{+}$T cell phenotype known as Th9 cells ${ }^{37}$. While IL-9 production was originally attributed to Th2 cells, identification of IL-9 expressing, IL-4 negative, and GATA3 low cells defined Th9 as a separate activation lineage ${ }^{70} . \mathrm{Pu} .1$ is the transcription factor that maintains the Th9 phenotype $\left.^{71}\right\}$. These cells have pathogenic roles in airway inflammation ${ }^{72}$, but have been shown to contribute to anti-helminth responses ${ }^{73}$ and the maintenance of innate cells in several inflammatory models ${ }^{74}$. The discovery that IL-1 family cytokines, along with TGF- $\beta$ could induce Th9 cells independently of IL-4 reinforces the independent status of Th9 cells ${ }^{75}$.

\subsubsection{T follicular helpers}

$\mathrm{T}$ follicular helpers $(\mathrm{Tfh})$ are a distinct subset of $\mathrm{CD}^{+} \mathrm{T}$ cells residing within the germinal centres of lymphoid organs and supporting B cell antibody production. Tfh express CXCR5, a chemokine allowing migration into the B cell follicle, programmed cell death protein 1 (PD-1), inducible T cell co-stimulator (ICOS) and IL-2176. With the discovery of the master transcription factor $\mathrm{Bcl} 6$, a transcriptional repressor that inhibits the expression of many genes through both epigenetic modifications and DNA binding, Tfh was resolved into a CD4+ $\mathrm{T}$ cell effector subset separate from Th1 or Th2 ${ }^{77}$. IL-21 and IL-6, both signalling via STAT3, can induce differentiation in vitro of CD4 ${ }^{+} \mathrm{T}$ cells with a Tfh phenotype ${ }^{78}$. Recently the Tfh subset has been divided in two with a population of cells expressing very high levels of CXCR5, PD-1 and ICOS being separated from the intermediate expressing cells. These high expressers have been labelled germinal centre Tfh (GC Tfh) as opposed to the intermediate expressing Tfh that appear to be located closer to the T-B cell border ${ }^{79}, 80$. Whether these are distinct differentiation states, or rather representative of a spectrum of phenotypes that cells can display depending upon activation state and signals, is yet to be delineated. Along with IL-2 1 Tfh cells can make moderate levels of cytokines usually associated with Th1, Th2 and Th17 cells ${ }^{39}$. Furthermore rather than Th2 cells, Tfh make up the majority of IL-4 producing cells in the $\mathrm{LN}$ during parasitic infections ${ }^{81}$. Tfh promote $\mathrm{B}$ cell responses, survival and isotype switching by mediating formation of the GC, producing supportive and isotype driving cytokines and expressing cell surface molecules such as CD40L and PD-1, which provide proliferative and pro-survival signals to the $\mathrm{B}$ cell ${ }^{82}$. Their importance in antibody production and their ability to co-express cytokines of the other 
Th phenotypes make these cells a confounding factor when assessing the roles of activated $\mathrm{CD} 4^{+} \mathrm{T}$ cell phenotypes in immune responses.

\subsubsection{Th2}

IL-4 is a strong promoter of Th2 differentiation but experiments demonstrating Th2 generation in IL-4 deficient mice indicate that it is not a required factor which mean the signals which drive Th2 differentiation in vivo remain unknown ${ }^{31,83}$. GATA3 is the master transcription factor of Th2 cells and instigates their production of the cytokines IL-4, IL-5 and IL-13, though IL-4 can be expressed independently of GATA3 in already differentiated Th2 cells ${ }^{84}$. These cells will become one of the major focuses of this thesis so more detail regarding differentiation, regulation and effector functions are covered in subsequent sections.

\subsubsection{Mechanisms influencing $\mathrm{CD}^{+} \mathrm{T}$ cell differentiation}

Activating $\mathrm{CD}^{+} \mathrm{T}$ cells in vitro in the presence of certain combinations of cytokines drives the generation of specific Th effector subsets. These data strongly influenced the view that it was the in vivo cytokine environment within which a cell was activated that would direct differentiation ${ }^{18}$. This theory was confounded by the fact that the cytokines required in vitro to generate certain Th phenotypes, were predominantly produced in vivo by the fully differentiated effector Th cells themselves, leading to a causality dilemma. Studies demonstrating that Th subsets could be generated in the absence of the in vitro driving cytokine lead to investigation into other potential signals that could initiate differentiation $^{31,85}$. In light of these data, evidence is mounting that rather than being the deterministic switches, signalling by some cytokines support selective outgrowth, and increase expression of genes that reinforce a particular Th phenotype.

\subsubsection{The contribution of dendritic cells to differentiation}

Antigen presenting DGs have been proposed to provide the deterministic signals that drive $\mathrm{CD} 4^{+} \mathrm{T}$ cell differentiation in vivo. Some studies show that specific subsets of DCs are predetermined to preferentially drive a particular activation state ${ }^{86,87}$. Other work however indicates that the signals DGs received prior to interacting with CD4 ${ }^{+} \mathrm{T}$ cells can change the DG to promote a certain Th effector phenotype ${ }^{88,89}$. In either case the route via which DGs dictate Th phenotype is either through the production of cytokines like IL-12, or through altered surface ligand expression changing the signalling through 
co-stimulatory receptors on the $\mathrm{CD}^{+} \mathrm{T}$ cell. $\mathrm{CD} 28$ is critical for optimal $\mathrm{T}$ cell activation but there are several families of co-stimulation receptors, which when signalled through, boost $\mathrm{T}$ cell activation and can modulate $\mathrm{CD} 4^{+} \mathrm{T}$ cell differentiation. CD70 expression is induced on stimulated DGs and can drive Th1 differentiation by signalling through the receptor CD27, the constitutive expression of which is increased on activated $\mathrm{CD}^{+} \mathrm{T}$ cells ${ }^{90,}{ }^{91}$. Significantly, CD27 signalling inhibited IL-17 production and Th17 differentiation via transcriptional and epigenetic regulatory pathways ${ }^{92}$. CD28 and CD27 signalling inhibit the generation of iTregs, and the absence of CD80, and CD40 on DGs enhances iTreg differentiation ${ }^{91}$.

OX40:OX40L interactions have long been proposed as a Th2 skewing pathway, with OX40L over-expressing mice being biased towards a Th2 phenotype, potentially by directly increasing IL-4 expression ${ }^{93,94}$. Recently OX40 signalling along with IL-4 and TGF- $\beta$ has been shown to be a potent initiator of IL-9 production and the Th9 phenotype $^{95}$. ICOS signalling was first associated with Th2 generation ${ }^{96}$ but further work has demonstrated that the induction of c-Maf leads to increased IL-4 and IL-21, which in turn induces Bcl-6 and CXCR5 on the activated CD4 ${ }^{+} \mathrm{T}$ cell and a Tfh phenotype ${ }^{97}$. One predominant theory states the signals the newly activated cells receive at the $\mathrm{T}$ and $\mathrm{B}$ cell zone border divides the closely related $\mathrm{Tfh}$ and $\mathrm{Th} 2$ phenotypes. CXCR5 and ICOS are induced by CD28, CD40 and OX40. If they bind to their ligands CXCR13 and ICOSL respectively, which are enriched in the B cell follicle, this may seal Tfh destiny and induce migration into the $\mathrm{B}$ cell zone ${ }^{98}$. Extended interaction with OX40L or IL-33-expressing border DG could promote Th2 differentiation and migration out of the $\mathrm{LN}$ instead ${ }^{99}$. With an increasing amount of literature indicating sub-populations, or particular activation states of DCs are critical for the generation of certain Th phenotypes, it is becoming clearer that the signals, both cytokine and cell-cell receptor mediated, coming from the DG are likely to be very important in determining activated $\mathrm{CD} 4^{+} \mathrm{T}$ cell phenotypes.

\subsubsection{The quantity and quality of $T$ cell receptor stimulation influences differentiation}

High affinity antigen-TCR interactions can lead to stronger initial signalling through the TGR, stronger binding and increased interaction time with the APG allowing paracrine cytokine signalling and contacts with co-stimulatory receptor ligands ${ }^{100}$. The 
same DGs presenting slightly different peptides can induce very different CD4 ${ }^{+} \mathrm{T}$ cell responses ${ }^{79}$.

Differing levels of infection in vivo, or antigen dose in vitro differentially can drive Th1 and Th2 responses. Infection with very low numbers of Leishmania major induced protective Thl responses in usually susceptible Balb/c mice, where as increasing the dose lead to Th2 induction ${ }^{101}$. In vitro studies utilising TCR transgenic cells cultured with ranged concentrations of peptide showed that very high levels of peptide lead to preferential Th2 differentiation. An intermediate peptide concentration strongly favoured Th1 while the balance would move towards Th2 differentiation again at very low peptide concentrations ${ }^{102,103}$. Antigen dose has also been implicated in the switch between Th1, Tfh and GC-Tfh, with an increasing dose of attenuated Listeria monocytogenes leading to a decrease in Thl and Tfh cells and subsequent increase in GC$\mathrm{Tfh}^{104}$. The expansion of GC-Tfh with high dose may just represent the increased availability of antigen to GC-B cells, which drives GC-Tfh activation by presenting peptide within the B cell follicle, though there are intracellular signalling pathways that may also explain this pattern. Increased TCR signalling induces interferon regulatory factor 4 (IRF4), which allows the expression of Blimp-1, a critical transcription factor for Th1, however, expression of IL-12R $\beta 2$ is inhibited at very high levels of TCR signalling ${ }^{79}$. This may explain the preferential differentiation towards Th2 and GC-Tfh, and away from Th1, seen with high antigen dose in in vitro and in vivo models respectively.

The sheer number of TCRs that are engaged with peptide-MHC II complexes is not enough to fully explain what drives $\mathrm{CD} 4^{+} \mathrm{T}$ cell differentiation, with many experiments indicating that the quality of the interactions must also have a role. The affinity a given TCR has for a peptide loaded MHC II molecule can significantly change the APC-T cell interaction, increasing the time the cells interact (dwell time) and thus increasing the signals a $\mathrm{T}$ cell receives ${ }^{105}$. One group of studies looked at a population of $50 \mathrm{CD} 4^{+} \mathrm{T}$ cells clones that were all responders to lysteriolysin O peptide from Listeria monocytogenes. When exposed in vivo to the peptide together, the polyclonal population produced activated $\mathrm{CD}^{+} \mathrm{T}$ cells with the ratio of 2:1:1 of Th1, Tfh and CG Tfh respectively. When the clones were analysed individually, they expressed very different differentiation patterns with some becoming all Thl or Tfh, and others with mixed phenotypes, however when the phenotypes were averaged they matched the original mixed population's ratios of 2:1:1 Th1, Tfh and GG Tfh ${ }^{104}$. This indicated that while 
$\mathrm{CD}^{+} \mathrm{T}$ cells might be able to respond to the same peptide presented by APCs in the same conditions, the differences in their TCRs can change the phenotypic outcome of the effector cells.

Differentiation driven by affinity between TCR and peptide-MHG II complexes has been demonstrated by measuring the responses of identical transgenic TCR clones to slightly altered peptides. P25 TCR transgenic mice have $\mathrm{CD} 4^{+} \mathrm{T}$ cells that are specific for a peptide from antigen $85 \mathrm{~B}$ (Ag85B), part of a complex of proteins that are the major secretion products from Mycobacterium tuberculosis ${ }^{106}$. Infected mice and humans have Ag85B reactive $\mathrm{CD}^{+} \mathrm{T}$ cells that are strongly polarised towards a $\mathrm{Th} 1$ phenotype $^{107}$. Immunisation with the 15 -mer P25 peptide induces strong I-A ${ }^{\mathrm{b}}$ restricted Th1 differentiation and expansion of V $\beta 11$ expressing CD4 ${ }^{+} \mathrm{T}$ cells, which were able to protect against subsequent challenge with M. tuberculosis ${ }^{108}$. TCR transgenic mice were generated from $\mathrm{P} 25$ reactive $\mathrm{CD} 4^{+} \mathrm{T}$ cell clones, and cells from these mice stimulated by P25 peptide-loaded APGs display Th1 differentiation in the absence of IL-12, IFN- $\gamma$ or any other polarising conditions ${ }^{109}$. It was found that decreasing the peptide concentration that the DCs were pulsed with did swing the P25 TCR transgenic cell responses to a more Th2 phenotype, as had been reported in other models ${ }^{109}$. Tamura et al wished to understand the role of the peptide in driving $\mathrm{CD} 4^{+} \mathrm{T}$ cell differentiation so they created an altered peptide (altered peptide ligand, APL) to compare to P25. The binding of the $\mathrm{P} 25$ peptide into the $\mathrm{I}-\mathrm{A}^{\mathrm{b}}$ cleft and the interaction with the transgenic TCR had been characterised, allowing targeted single amino acid modification from glutamic acid to alanine, ensuring the altered peptide maintained its MHC II binding characteristics but decreased the affinity of the TCR. Then they compared the in vitro differentiation of P25 TCR transgenic cells stimulated with DGs pulsed with the same concentration of each of the peptides and found that, unlike the P25 driven Th1, the APL induced Th2 polarisation. ${ }^{106}$ The phenotype of DGs pulsed with either of the ligands were not reported to be significantly different so it was inferred that the alteration in TCR affinity was the sole driver of the differential polarisation by the two peptides.

\subsubsection{Multiple signals drive differentiation}

The understanding of what drives differentiation of $\mathrm{CD}^{+} \mathrm{T}$ cells into the range of effector subsets recognised today has significantly evolved over the last two decades. From initial studies which demonstrated that the cytokine milieu determined effector 
function, to demonstrations that different DC subsets, or the expression of costimulatory molecules by APGs, were critical, and work comparing the contribution of TCR affinity, it appears likely that no single factor is the switch that decides a CD4 ${ }^{+} \mathrm{T}$ cell fate. It is highly probable a complex matrix of factors, with certain combinations of signals preferentially driving differentiation towards one of the Th phenotypes.

A recent study by Van Panhuys et al. has attempted to compare the relative contribution by several different factors in determining the difference between Th1 and Th2 differentiation. This study assessed in vivo responses by TCR transgenic CD4 ${ }^{+} \mathrm{T}$ cells specific for pigeon cytochrome $\mathrm{C}$ peptide (pPPC) to pPPG loaded DCs exposed to Th1 skewing adjuvants lipopolysaccharide (LPS) and CpG oligodeoxynucleotides (CpG) or Th2 skewing papain or Schistosomal egg antigen (SEA) prior to adoptive transfer. The CD4 ${ }^{+} \mathrm{T}$ cells spent a longer time interacting with Th1 skewed DCs than Th2, and the expected cytokine profiles (IFN- $\gamma$ from Th1 and IL-4 from Th2) were induced by each of the adjuvant treated DCs. It was found that Th1 stimulated DCs expressed higher levels of CD80, and blocking antibodies against this co-stimulatory ligand led to Th1 skewed DGs inducing increased CD4 ${ }^{+}$T cell IL-4, decreased IFN- $\gamma$ and having decreased interaction times with the $\mathrm{CD} 4^{+} \mathrm{T}^{+}$cells. ${ }^{89}$

The antigen dose the DCs were loaded with can drastically change the CD4+ ${ }^{+} \mathrm{T}$ cell phenotype irrespective of the adjuvant DGs are treated with. Transgenic CD4+ ${ }^{+} \mathrm{T}$ cells exposed to Th1 or Th2 skewed DGs pulsed with low peptide concentrations showed a significant expression of IL-4, while increasing the dose of peptide lead to a proportional increase in IFN- $\gamma$ expression and decreased IL-4. The effect of peptide dose was considerable with low peptide concentrations inducing equal IL-4 and IFN- $\gamma$ even from Th1 skewed DGs, and high peptide Th2 skewed DCs inducing much more IFN- $\gamma$ than IL-4. The increase of peptide dose also had a significant effect on the expression of IL$12 \mathrm{R} \beta$ only 24 hours after stimulation. This increase was even more exaggerated with the Th1 skewed DCs, though it was still present in the Th2 group. ${ }^{89}$ This pathway could be a mechanism through which Thl differentiation is perpetuated in high antigen exposed CD4 ${ }^{+} \mathrm{T}$ cells. This study found that antigen dose was a strong mediator of $\mathrm{CD}^{+} \mathrm{T}$ cell differentiation, although in situations of equal TCR stimulation, adjuvants could drive differential co-stimulation ligand expression by DCs, which also preferentially induced different Th phenotypes. Finally, increased TCR stimulation lead to cells being more sensitive to their cytokine milieu, potentially allowing reinforcement 
of a certain phenotype if the right environmental signals are present. ${ }^{89}$ Although far from complete, this research effectively compares different signals in a very controlled system, and further studies will need to consider the full context of $\mathrm{T}$ cell stimulation while assessing the contributions of certain signals to differentiation.

\subsection{T helper 2 cells; orchestrators of Type 2 immunity}

The identification of Th2 as a specialised phenotype of activated CD4 ${ }^{+} \mathrm{T}$ cells lead to the association of these cells with immune responses against particular infections ${ }^{110,111}$, but also in diseases caused by overactive immune responses ${ }^{112}$. Th2 cells are distinguishable by their expression of IL-4, IL-5, and IL-13 and the master transcription factor GATA3, however other genes are also closely related to Th2 function ${ }^{113}$. IL-3 has long been associated with Type 2 immune responses ${ }^{114}$ and mediates basophilia and the development of mast cells and eosinophils ${ }^{115}$. IL-9, which is also important for inducing mastocytosis was once attributed to Th2 cells but identification of the closely related Th9 cells and ILCs means most IL-9 production is now considered Th2 independent ${ }^{116}$. The dual roles of Th2 cells in protection and pathology have been widely studied, and while many of their specific functions have been characterised in a variety of models it is still not known what differentiates a pathogenic from a protective Type 2 immune response.

\subsubsection{Protective roles for Th2 cells}

Initial experiments using Leishmania major and Trichinella spiralis infection models found that strains of mice that displayed resistance to infection tended to induce a Th1 phenotype, while production of Th2 cytokines was associated with susceptibility to infection $^{117,118}$. However for many of the parasitic worms known as helminths, Type 2 immune responses were critically important for the protection observed in mice receiving a second infection challenge. Type 2 immune responses were shown to be able to disrupt the life cycles, and pathology associated with infection of model nematodes, trematodes and cestodes, though the mechanisms controlling these infections varied with the specific parasites ${ }^{119}$.

The production of the IgE antibody requires both $\mathrm{CD}^{+} \mathrm{T}$ cells and IL-4, and the driving of class switching B cells to IgE production was long attributed to Th2 cells, however the recent discovery of Tfh as the major IL-4 producer in LNs has changed 
this interpretation ${ }^{120}$. Despite its roles in increasing survival and activation of basophils and mast cells through FceRI ligation ${ }^{121}$, the role for $\operatorname{IgE}$ in the expulsion of, or protection against intestinal helminths remains controversial ${ }^{122,} 123$.

The lumen of the intestines are a desirable place for a parasite to reside, with ample access to nutrients, relative shielding from direct attack by host immune responses and an easy way to disseminate eggs; many helminth species spend at least part of their life cycle within the gut. Th2 responses play a critical role in expulsion of these gut residing helminths. Nippostrongylus brasiliensis is a nematode that follows an infectious route common to many human and rodent hookworms. Larvae penetrate the skin then travel through currently undetermined routes to the lung parenchyma where the larvae undergo a moult and travel via the mucociliary ladder up the trachea then down into the small intestines, where the adult worms mate and produce eggs. ${ }^{124}$ In a primary infection Th2 derived signals are required for expulsion of these worms from the gut; the absence of $\mathrm{CD} 4{ }^{+} \mathrm{T}$ cells, either in $\mathrm{Rag} 1^{-/}$or antibody depleted mice, allows worms to remain in the gut for a significantly longer time ${ }^{14,125,126}$. The loss of expulsion with $\mathrm{CD} 4{ }^{+}$depletion can be largely rescued by the addition of Th2 cytokines IL-4 and IL13 ${ }^{14,126}$. However, a recent discovery found that T cell specific IL-4 ${ }^{-/-}$and IL-13 $3^{-/-}$mice have normal expulsion of worms, while global IL-4 $4^{-/-}$and IL-13 $3^{-/-}$mice have a significant number of the worms surviving in the intestine at day 9 post-infection ${ }^{127}$. So while $\mathrm{CD}^{+} \mathrm{T}$ cells may be important for $\mathcal{N}$. brasiliensis expulsion, they may be recruiting another population of Type 2 cytokine producing inflammatory cells.

The immune response initiated against Trichuris muris depends on the strain of mouse infected; the preferential differentiation of Th1 or Th2 CD4+ $\mathrm{T}$ cells dictates whether a given strain will be susceptible or resistant to long term infection ${ }^{128}$. Mice ingest Trichuris muris eggs, which hatch in the ileum and larvae migrate to the proximal colon and caecum, there they burrow into the gut wall, constructing tunnel-like structures consisting of dead epithelial cells and actin ${ }^{129}$. Strains of mice that are resistant to the $\mathcal{T}$. muris infection induce strong Th2 responses that mediate expulsion of the worms ${ }^{130}$. The direct mechanisms that drive T. muris infection are unknown but as it burrows into the gut tissue, similar to Heligmosomoides polygyrus, there are likely to be shared pathways that dictate protective responses.

Heligmosomoides polygyrus larvae have a free-living stage after hatching, however instead of infecting via skin penetration $H$. polygyrus requires ingestion to initiate infection. Once in the small intestine $H$. polygyrus larvae rapidly invade the wall of the intestines where they 
undergo two moults, emerging approximately 8 days later into the lumen of the intestine as adults. Primary infection establishes a chronic infection, with the length of infection mouse strain dependent, ranging from 4 to 20 weeks. ${ }^{124} \mathrm{~T}$ and $\mathrm{B}$ cell deficient mice display prolonged infections, CD4 depletion leads to increased fecundity (a read out of worm health), whereas adoptive transfer of $\mathrm{CD}^{+} \mathrm{T}$ cells to naïve mice can decrease the length of infection ${ }^{114}$. Whilst Type 2 immune responses play a role in resolution of the primary infection, it is in a secondary reaction that the Th2 cell is truly important. Mice that have had their primary infection cleared after two weeks by treatment with anti-helminthics, will efficiently clear a secondary infection within two weeks of reinfection ${ }^{110}$. The clearance of the secondary infection requires responses by memory Th2 cells and macrophages in the granulomas that form around the larvae once they bury into the gut wall of an immune individual. Th2 cells maintain the macrophages in an alternately activated phenotype that is critical for causing the worm stress and decreasing worm survival. ${ }^{131}$

$\mathcal{N}$. brasiliensis primary infection is transient in immune-competent mice and the strong Th2 driven immune responses generated are able to provide protection against secondary challenge, with up to $90 \%$ of worms being killed before they can migrate to the intestines and become adults ${ }^{132}$. The mechanism of killing and the location where this protection occurs is still uncertain, though strong evidence indicates the lung immune environment may play a significant role in protection. Protection against secondary infection is diminished in $\mathrm{CD}^{+} \mathrm{T}$ cell deficient $\mathrm{MHC} \mathrm{II}^{-/-}$mice and in mice deficient for the intracellular signalling molecule downstream of the IL-4 and IL-13 receptors, signal transducer and activator of transcription 6 (STAT6) ${ }^{132}$. Using TCR transgenic animals with varying proportions of their $\mathrm{CD} 4^{+} \mathrm{T}$ cell repertoire consisting of $\mathcal{N}$. brasiliensis non-responding cells it was demonstrated that bystander activation was uncommon, with none of the $\mathrm{CD}^{+} \mathrm{T}$ cells with specific transgenic TCRs for either ovalbumin (OVA) or lymphocytic choriomeningitis virus (LGMV) peptides becoming Th2 differentiated, despite measurable responses from CD4 ${ }^{+} \mathrm{T}$ cells not expressing the restricted TCR within the same tissues. These experiments also found that protection was inversely proportional to the fraction of $\mathrm{CD} 4^{+} \mathrm{T}$ cells that had restricted TCRs; the more unrestricted TCR carrying $\mathrm{CD} 4^{+} \mathrm{T}$ cells the greater the protective response. In mice where their entire $\mathrm{CD} 4^{+} \mathrm{T}$ cell population consisted of cells carrying a restricted TCR there was a total loss of protection. ${ }^{133}$ 
Harvie et al. targeted the lung as the principal site for Th2 priming in eloquent experiments where mice were initially infected subcutaneously (for skin and lung priming), intranasally (i.n.) (lung priming) or by oral gavage (gut priming) with developmentally appropriate larvae for each site. The skin and/or lung primed mice were treated with anti-helmenthics to kill the worms before they could effectively migrate to the gut. These primed mice were then challenged subcutaneously and the numbers of worms in the lung on day 2, or the gut on day 6-post infection enumerated to assess the ability of mice primed at different locales to protect against the worms. These experiments demonstrated that only mice that were primed by infection passing through the lung had effective protection and the ability to kill worms early in the infection. Mice with both skin and lung priming had the same level of protective responses as those that were just primed via the lung, indicating the skin is not a strong site of protective responses. Mice that had only received gut priming had the same number of intestinal larvae as the other primed mice indicating that they could expel intestinal worms efficiently, but they had high worm counts at day 2 in the lung indicating gut priming did not provide systemic protection. ${ }^{132}$ This study was followed by a publication that demonstrated that adoptive transfer of CD4+ ${ }^{+} \mathrm{T}$ cells from the lung of a primed mouse could provide protection to naïve mice, and signalling through IL$4 \mathrm{R} \alpha$ was required to induce protection ${ }^{134}$. While earlier papers had demonstrated the $\mathrm{T}$ cell specific IL-4R $\alpha$ expression was required for optimal $\mathcal{N}$. brasiliensis induced cytokine responses ${ }^{135}$, it was then demonstrated using FTY720, a sphingosine 1 phosphate receptor agonist which blocks LN egress of naïve lymphocytes, that a population of IL4R $\alpha$ dependent lung resident $\mathrm{CD} 4^{+} \mathrm{T}$ cells responded to $\mathcal{N}$. brasiliensis infection and were sufficient for providing protection ${ }^{134}$. These data demonstrate that lung Th2 responses are essential for protecting against $\mathcal{N}$. brasiliensis infection, though how the worms are arrested and killed remains to be demonstrated.

\subsubsection{Pathogenic roles for Th2 cells}

Th2 activation, when targeted towards killing or expulsion of parasites has obvious benefits for the host, however, Th2 cell responses also mediate the development of allergic diseases and the associated detrimental pathologies. Allergic diseases arise when the immune system responds to an innocuous antigen. The overt immunological response can cause significant damage to tissues often impairing function and 
occasionally leading to potentially lethal systemic anaphylactic reactions ${ }^{19}$. These immune responses are predominantly of a Type 2 phenotype, sharing many features of an anti-parasite response including Th2 differentiation, IgE production, accumulation of mast cells, basophils and eosinophils, and mucus production ${ }^{136,137}$. For experimental models of allergic disease two phases are required; a sensitisation phase, which initiates the Type 2 response often at a systemic level ${ }^{138}$. This is followed by a challenge, which accumulates the necessary mediators in the particular tissue and initiates the pathology 137 . While it is unknown if this is how allergies are generated in humans, these sensitisation/challenge models produce pathologies that mirror many of the salient features of the human diseases.

Asthma is a term used to cover a number lung diseases with a range of causes and phenotypes, however allergic asthma is a form of the disease associated with Type 2 immune responses towards a single, or group of trigger antigens ${ }^{139}$. Allergic asthma's most significant characteristic is eosinophilia in the lungs particularly in the bronchialalveolar lavage fluid $(\mathrm{BALF})^{140}$. In murine models of allergic asthma mice are commonly sensitised against an allergen then challenged with the same antigen either intranasally or with inhaled aerosolised antigen ${ }^{141}$. The lungs undergo significant pathological changes after challenge, with infiltrations of a range of leukocytes, particularly eosinophils and mucus production that can cause obstruction of airways in severely asthmatic individuals ${ }^{142}$. An objective read out of asthma severity is airways hyperreactivity (AHR), this can be assessed by administration of a non-specific bronchoconstrictive stimulant such as acetylcholine or methacholine. Subsequently, lung function changes can be measured by using whole-body plethysmography, or measuring pulmonary resistance and dynamic compliance in tracheotomised mice. ${ }^{141}$ Mice with AHR will have far greater bronchoconstriction compared to baseline or naive mice. This is due to increased responsiveness of smooth muscle cells to the bronchoconstrictive stimulant ${ }^{141}, 142$. The direct mechanisms priming the smooth muscle cells are unknown, but both nervous and cytokine signalling appear to play a role ${ }^{143,144}$. Eosinophilia in the lung can be decreased by antibody neutralisation of the $\mathrm{Th} 2$ cytokine IL-5, or by simultaneous disruption of IL-4 and IL-13 signalling ${ }^{145}$. AHR and mucus production require the priming and presence of Th2 cells, as does antigen specific IgE production ${ }^{146}$.

Food allergy demonstrates many of the same the features and requirements as allergic asthma. Th2 dependent IgE production induced by sensitisation is required for the 
development of disease ${ }^{147}$. Mast cells infiltrate the gut and are primed to release anaphylaxis-inducing factors including histamines and mast cell proteases upon antigen binding and cross-linking of FceRI bound IgE ${ }^{148}$. Th2 (and Th9) associated cytokines IL-3 and IL-9 are proposed to be important for mucosal mastocytosis ${ }^{149}$. IgE loaded mast cells and basophils in the skin are also proposed to be important for wheal and flare reactions during localised exposure to an allergen ${ }^{150}$. Allergic skin diseases such as atopic dermatitis and eczema can also have Th2 dependent eosinophilia, which often correlates with severe pathology. Th2 cells in atopic dermatitis models have been shown to produce the cytokine IL-31 that potentially increases the itch response leading to increased damage to the skin and further inflammation. ${ }^{151}$

Atopic diseases including allergic asthma, rhinitis, food allergies and atopic dermatitis are caused by dysregulated tolerance leading to differentiation of Th2 cells that choreograph the pathogenic responses in the effected tissues. While the exact reason these immune responses are initiated is still unknown significant research has been focussed on blocking the downstream pathogenic Type 2 responses that mediate pathology.

\subsubsection{Immunopathologies as side effects of protective Type 2 responses against helminths}

The Type 2 immune response is associated with wound healing and the resolution of damage. While in some situations Type 2 responses induce appropriate healing responses, in others the overactive immune system causes more damage than what would have occurred in the absence of a response. Th2 cell responses in the lung are critical for mediating protection against a secondary infection, they are also important for limiting and resolving the damage caused by the invading parasites ${ }^{119}$. As reported by Chen et al. when $\mathcal{N}$. brasiliensis enter the lung they cause a large amount of damage resulting in haemorrhaging into the airways. IL-17 driven responses can recruit neutrophils that increase the number of red blood cells (RBCs) in the BALF. Both Th2 cytokines IL-4 and IL-13, signalling through IL-4 receptor $\alpha$ (IL-4R $\alpha$ ), redundantly opposed the Th17 induction, limiting neutrophilia and decreasing haemorrhaging. Macrophages activated by Th2 signals were critical to both clearance of RBCs from the airways and limiting inflammation, their depletion or functional inhibition leading to significantly greater inflammation in the alveolar spaces. ${ }^{152}$ 
After a primary $\mathcal{N}$. brasiliensis infection has passed through the lungs a long-term response is initiated which leads to major remodelling of the lung architecture, resulting in AHR and an emphysema-like phenotype. Marsland et al. have shown that the remodelling appears permanent, having been measured out to 300 days following infection $^{153}$. The excreted products of the $\mathcal{N}$. brasiliensis larvae were sufficient to induce AHR but not emphysema, signifying potentially divergent mechanisms, with an inflammatory cause for the AHR, while infection related damage may cause the emphysema development. IL-13\% animals were slightly protected from developing AHR, while IFN ${ }^{-/-}$mice displayed more severe AHR after infection, hinting that the Th2 cytokines may play a role. This correlates with data demonstrating high levels of Th2 cytokines in the lungs over 36 days post-infection. However, IL-13 ${ }^{-/-}$and IL-4R $\alpha^{-/-}$ mice both developed the emphysema phenotype concluding that neither IL-4 nor IL-13 was the driving cytokine for the lung remodelling. ${ }^{153}$ It has been suggested that the remodelling events may be part of the protective mechanism, making it more difficult for subsequent infections to transit the lung, though this remains to be evaluated. So while Th2 responses are initially protective in limiting haemorrhage, they can then contribute to the development of post-infection AHR, giving them a mixed role in the lung during primary infection.

Schistosoma mansoni is a trematode, or blood fluke, that infects both mice and humans. The adult worms live in the blood and immune responses generated against them are initially Thl polarised, but once a mating pair of adults begin releasing eggs a very strong Th2 shift occurs. The released eggs pass through the liver on their way to the intestines or bladder, they can become lodged in the sinusoids of the liver where a strong Th2 driven immune response generates a granuloma to wall off the egg. ${ }^{154}$ In the absence of IL-4 signalling a pathogenic, largely Th1 mediated, inflammation develops in both the liver and the intestine, which could lead to lethal septic shock. In the presence of sufficient regulatory pathways largely controlled by IL-10 and macrophages, the granulomatous response is kept under control. ${ }^{155}$ In the absence of regulation IL-13 driven fibrosis can significantly compromise liver function ${ }^{156}$. This represents another situation where Th2 responses are the balance between regulation and pathology; while Th2 cytokines control Th1 driven inflammation, which may be lethal for the Schistosoma mansoni infected host, IL-13 also drives the development of fibrosis leading to long-term liver damage. 


\subsection{Functions and expression of IL-4 and IL-13}

IL-4 and IL-13 mediate many of the down stream effector functions of the Type 2 immune response; although these two cytokines share many common features they have been found to have very divergent roles in vivo. IL-4 and IL-13 are closely related cytokines, their genes are adjacent to each other on mouse chromosome 11 (human chromosome 5$)^{157}$. Both proteins have a structure largely consisting of $\alpha$-helices and use the IL-4R $\alpha$ chain as part of their receptor complexes ${ }^{158}$. They have many overlapping functions in Type 2 immune responses. IL-4 was originally identified as a B cell stimulating factor, shown to promote proliferation of activated B cells ${ }^{159}$ and class switching to the $\operatorname{IgG} 1$ isotype ${ }^{160}$. It was soon shown that $\mathrm{T}$ cells could produce IL-4 and upon the realisation that Th1 and Th2 clones could be identified by IFN- $\gamma$ and IL-4 respectively, IL-4 became the signature cytokine of Th2 activation ${ }^{28}$. IL-13 was identified as a very closely related cytokine sharing approximately 30\% sequence homology with IL-4 ${ }^{161}$. IL-13 was subsequently shown to be able to induce many of the same immune responses as IL-4, including driving the class switching of human B cells to $\operatorname{IgG} 4$ and $\mathrm{IgE}$ production ${ }^{162}$, although the generation of genetic knock-out and neutralising antibody reagents for IL-13 delineated non-redundant functions in a range of models. 


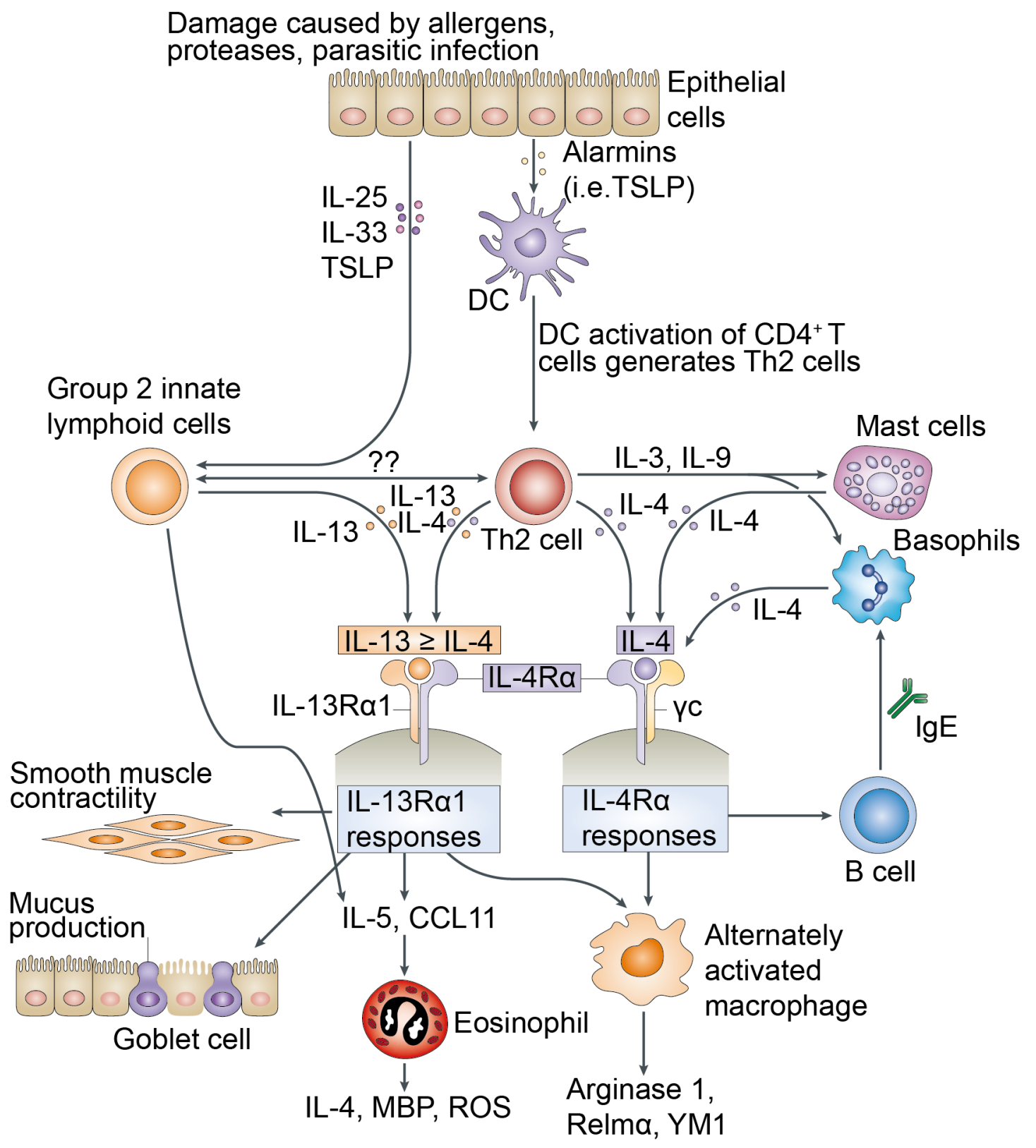

Figure $1.2-$ CD4 $^{+} \mathrm{T}$ cells and IL-4/IL-13 signalling are central to Type 2 immune responses.

Damaged epithelial cells release cytokines including IL-25, IL-33 and TSLP that drive migration and activation of DCs to the $\mathrm{LN}$ where they present antigen and drive differentiation of Th2 cells. Epithelial derived cytokines also activate ILC2s within the tissues. IL-4 and IL-13 from ILC2s, mast cells, basophils and Th2 differentiated CD4 ${ }^{+} \mathrm{T}$ cells signal through the type I and type II receptors. IL-4 drives IgE isotype switching by B cells through the type I receptor (IL-4R $\alpha$ and $\gamma$ c). IL-13 has higher affinity for the type II receptor complex (IL-4R $\alpha$ and IL13R $\alpha 1$ ) than IL-4; signals through this receptor promote eosinophilia, goblet cell mucus production and smooth muscle contractility. Macrophages responding to IL-4 and IL-13 through both receptor complexes express an alternate activation phenotype associated with wound healing and protective responses against helminths. 


\subsubsection{IL-4 and IL-13 receptor complexes}

The receptor for IL-4 is widely expressed on both haematopoietic and nonhaematopoietic cells ${ }^{163}$. The receptor consists of a heterodimer of IL-4R $\alpha$ and the $\gamma_{c}$, which is shared by a large number of cytokine receptors including IL-2, IL-7, IL-9, IL15 and IL-2 $1^{164}$. Ligation of IL-4 to the receptor triggers two intracellular signalling pathways, one via STAT6 and another that phosphorylates insulin receptor substrate 2 (IRS2) and can cause activation of the $\mathrm{PI}_{3}$ and MAPK pathways. IRS2 triggering may be important for myeloid responses to IL-4, though lymphoid cells appear to rely completely on the STAT6 pathway. ${ }^{165}$ Naïve lymphocytes express about 300 IL-4R $\alpha$ molecules and upon activation this number increases between 5-10 fold, increasing their sensitivity to IL-4, which can reinforce Th2 phenotype differentiation of CD4+ ${ }^{+} \mathrm{T}$ cells or class switching by B cells ${ }^{166}$. T cell expression of IL-4R $\alpha 1$ is tightly regulated, IL-2 signalling induces upregulation but once the TCR is engaged and begins signalling, expression of IL-4R $\alpha 1$ is negatively regulated, leaving only a small window just before and during early antigen recognition that $\mathrm{CD}^{+} \mathrm{T}$ cells can respond to initial IL-4 signalling. Once STAT6 is phosphorylated transcription of IL-4R $\alpha 1$ and IL-4 is stably increased. ${ }^{165}$

IL-13 also utilises the IL-4R $\alpha$ subunit for its receptor, but replaces the $\gamma \mathrm{c}$ with a specific IL-13R $\alpha 1$ subunit. IL-4 can also bind this receptor complex ${ }^{167}$. The sharing of receptors and the relative abundance of the subunits on different cell populations has exposed some interesting biology about these two cytokines. The $\gamma_{c}$ is expressed preferentially on haematopoietic cells, so non-haematopoietic cells respond to both cytokines via the type II receptor (IL-4R $\alpha /$ IL-13R $\alpha 1)^{168}$. The affinity of IL-13 for the IL-13R $\alpha 1$ chain is far lower than IL-4 for the IL-4R $\alpha\left(\mathrm{K}=3 \times 10^{7} \mathrm{M}^{-1}\right.$ and $10^{10} \mathrm{M}^{-1}$ respectively), but the IL-4/IL-4R $\alpha$ complex has about 25 times lower affinity for the IL$13 \mathrm{R} \alpha 1$ chain that the IL-13/IL-13R $\alpha 1$ complex does for IL-4R $\alpha$ chain ${ }^{158,}{ }^{169}$. Greater amounts of IL-13 are required to saturate initial receptor binding, but once bound there is a higher level of signalling induced by IL-13 than IL-4. So, while IL-4 reaches peak signalling at lower concentration, provided there is enough IL-13 available it can induce a higher maximum signal via the type II receptor.

IL-13 can bind to the separate IL-13R $\alpha 2$ chain ${ }^{170}$, which adds complexity when attempting to correlate the amount of IL-13, produced and potential downstream 
functions. This IL-13R $\alpha 2$ chain has a four-fold higher affinity for IL-13 than the IL$13 \mathrm{R} \alpha 1$ and with no obvious intracellular signalling motifs identified it has been proposed to act as decoy receptor, regulating the functions of IL-13 ${ }^{171}$. Soluble IL$13 \mathrm{R} \alpha 2$ has also been detected, this soluble receptor is a potent inhibitor of in vivo IL-13 function $^{172}$. The production of IL-13R $\alpha 2$ can be driven by IFN- $\gamma$, IL-17 and TNF- $\alpha$, indicating a potential regulatory pathway via which Th17 cells could attenuate Th2 effector function ${ }^{173,174}$. Knock-out mice deficient for the IL-13R $\alpha 2$ chain had increased levels of tissue IL-13 and decreased serum levels, indicating that the IL-13R $\alpha 2$ may have a role in trafficking IL-13 to the serum or stabilising it there. Macrophages also had diminished NO responses to LPS in the absence of the receptor. ${ }^{171}$ While there is still relatively little known about the role of IL-13R $\alpha 2$, it is an important factor to consider when assessing the role of IL-13 function.

\subsubsection{Lymphocyte responses to IL-4 and IL-13}

The generation of an IL-4 neutralizing antibody and an IL-4 knock-out mouse allowed assessment of the role for IL-4 in vivo. The near complete inhibition of IgE production and diminished IgG1 levels in these models demonstrated the importance of IL-4 for B cell function ${ }^{175,176}$. Other than the requirement for STAT6 signalling, little is known about what signals mediate $\mathrm{B}$ cell switch to $\mathrm{IgE}$, or the potential life cycle and location of $\mathrm{IgE}$ switched cells ${ }^{120}$. IgE binds to the high affinity receptor FceRI, and the low affinity FceRII (CD23) receptor. FceRI is highly expressed on mast cells and basophils, and these cells collect serum IgE, which stabilises them and increases their survival. When the bound $\mathrm{IgE}$ comes in contact with cross-linking antigen, signalling through FceRI causes cellular activation, cytokine production and degranulation. ${ }^{121}$ FceRII is expressed on $\mathrm{B}$ cell surface and can regulate the production of $\operatorname{IgE}^{120}$. Populations of macrophages and DGs can express both Fce receptors, which mediate uptake and processing of bound IgE-antigen complexes ${ }^{177}$. Despite the robust production in response to parasitic infection, there is mixed evidence of a role for $\operatorname{IgE}$ in most experimental helminth infection models ${ }^{122,123}$. Production of $\operatorname{IgE}$ against an innocuous antigen can have dire consequences, as seen in allergies, where the production of allergen specific $\operatorname{IgE}$ is required for the development of both localised and systemic anaphylaxis ${ }^{178}$. 
IL-13 was originally shown to drive isotype switching of human B cells, however murine B cells do not express the IL-13R $\alpha 1$ chain so are directly unresponsive to IL-13 ${ }^{179}$. Despite this IL-13 is important for mouse B cell effector function in vivo. The development of IL-13 over-expressing transgenic mice, and exposure of wild-type (WT) mice to recombinant IL-13, increases serum levels of IgE ${ }^{180}$. IL-13R $\alpha 2$ knock-out mice, with their higher levels of tissue IL-13 also exhibit increased serum levels of IgE, IgA, IgG2a and IgG2b antibody isotypes ${ }^{171}$. Mature T cells in both mice and humans do not express the IL-13R $\alpha 1$ chain, so are also unable to respond to IL-13, however CD4+ $\mathrm{T}$ cells from IL-13 knock-out mice demonstrated diminished Th2 responses (as measured by production of IL-4 and IL-5 expression) both in vivo and with in vitro stimulation. This also correlated with diminished antigen specific $\mathrm{IgE}$ production in response to sensitisation with OVA. ${ }^{181}$ The fact that IL-13 addition to cultures could not rescue Th2 responses of $\mathrm{CD}^{+} \mathrm{T}$ cells from IL-13 deficient animals indicates that IL-13 may not be directly acting on lymphocytes but may be modulating the function of another cell population that is important in lymphocyte development or responsiveness.. This was supported by apparent changes in thymic development observed in IL-13 overexpressing transgenic mice ${ }^{180}$. However, it was also discovered that the deletion of the Il13 gene in mice also disrupted the expression of the adjacent Il4 gene, potentially explaining their generally decreased Type 2 responses ${ }^{182}$. So while IL-13 dependent changes in IgE production may be explained by decreased $\mathrm{CD} 4^{+} \mathrm{T}$ cell availability and function or defective IL-4 expression in IL-13-/- mice, how mouse T cells and B cells are responding to application of recombinant IL-13 remains to be explained.

\subsubsection{Alternate activation of macrophages}

Macrophages respond to IL-4 and IL-13, which both drive the development of an alternate activation phenotype, designated M2a, which is markedly different to IFN- $\gamma$ or toll-like receptor (TLR) stimulated, classically activated M1 macrophages ${ }^{5}$. Macrophages express the IL-13R $\alpha 1$, which means they can respond to both IL-4 and IL-13 ${ }^{183}$. Juntrila et al. demonstrated that macrophages unlike non-haematopoietic cells, which rely on the type II receptor, macrophages also express the $\gamma \mathrm{c}$, which modifies their relative sensitivities to the two cytokines. Any cell with high abundance of $\gamma \mathrm{c}$ and IL-4R $\alpha$ will respond more strongly to IL-4 than equal concentrations of IL-13, regardless of IL-13R $\alpha 1$ expression. If either $\gamma$ c or IL-13R $\alpha 1$ expression is limited, IL-4 
driven signalling is significantly diminished. The limiting factor on IL-13 driven signalling is the initial binding. Once present in sufficient concentrations to bind, IL-13 efficiently induces strong recruitment of receptor chains and signalling. ${ }^{168}$ So while IL-4 signalling is dependent upon levels of receptor chain availability, IL-13 more heavily relies on reaching a critical concentration.

M2a phenotypes decrease the production of nitric oxide and express a particular array of chitinases. Arginase-1 (Arg1) expression is a defining feature of M2a macrophages, and it is an enzyme that directly opposes a crucial pathway utilised by M1 macrophages $^{5}$. Inducible nitric oxide synthase (iNOS) is an M1 associated enzyme that converts L-arginine to nitric oxide (NO) and L-citrulline, Argl depletes the L-arginine availability, converting it instead to L-ornithine, which can have roles in mediating the metabolic functions of the M2a macrophages ${ }^{184}$, and potentially in killing helminths ${ }^{122}$. Consumption of L-arginine can have a negative effect on local $\mathrm{T}$ cell proliferation and may also be a mechanism of suppressing $\mathrm{T}$ cell responses ${ }^{184}$.

M2a macrophages express the mannose receptor (MR, CD206), which potentially mediates the binding of M2a macrophages to carbohydrate rich targets ${ }^{185}$. While MR expression has been inferred to allow macrophages to endocytose glycoproteins for processing and presentation, the decreased phagocytosis measured in M2a macrophages could diminish the importance of this MR role. Chitinase expression, particularly Ym-1 and Ym-2, are significantly increased by macrophages in response to IL-4 and IL-13 signalling. Chitin is found in insects and fungi, however the Type 2 associated upregulation of $\mathrm{Ym}-1$ and $\mathrm{Ym}-2$ lead to theories about the roles these enzymes might have in controlling helminths, which both produce chitin and induce strong IL-4 and IL-13 expression during infection ${ }^{186}$. Resistin-like molecule $\alpha(\operatorname{Relm} \alpha)$ is another protein characteristically expressed by M2a macrophages, Relmo can mediate extracellular matrix deposition but also appears to have roles in supressing $\mathrm{CD}^{+} \mathrm{T}$ cell effector functions ${ }^{187}$. M2a macrophages occupy a conflicting position within Type 2 immunity, while they are strongly induced by Type 2 cytokines and secrete products that may directly effect invading parasites, they also display regulatory roles and may be part of a negative feedback loop keeping the inflammatory response in check. Identifying macrophages expressing the M2a activation phenotype has proven difficult, early studies found that the MR can act as a surrogate for identification but 
more recent studies have required the expression of multiple identifying molecules including Arg-1, Relma and phosphorylated STAT6 (pSTAT6) ${ }^{5}$.

\subsubsection{Responses by non-haematopoietic cells to IL-4 and IL-13}

Both IL-4 and IL-13 are expressed by Th2 cells in non-lymphoid tissue during Type 2 inflammation, however many of the responses seen by non-haematopoietic cells are attributed to IL-13 rather than IL-4 signalling. This may be due to differences in the relative abundance of the two cytokines but this has yet to be conclusively shown. Through the use of cytokine, receptor and STAT6 knock-out mice IL-4 and/or IL-13 have been shown to be important for leukocyte chemotaxis, mucus secretion, smooth muscle hyper contractibility, fibrosis, and tissue remodelling ${ }^{188}$.

Through the IL-4R $\alpha /$ IL-13R $\alpha 1$ type II receptor both IL-4 and IL-13 are able to induce the expression of vascular cell adhesion molecule-1 (VCAM-1) on endothelial cells, encouraging the diapedesis of activated leukocytes from circulation into the parenchymal tissue ${ }^{189}$. Comparison of WT mice to those with a genetic deletion of the IL-13R $\alpha 1$ chain demonstrated several significant roles these receptors play in inflammatory models. Loss of the IL-13R $\alpha 1$ chain means IL-13 cannot signal at all and IL-4 will no longer be able to signal on cells not expressing the $\gamma \mathrm{c}$, regardless of the presence of the IL-4R $\alpha$. The liver pathology of Schistosoma mansoni infection has been associated with Type 2 immune responses ${ }^{154}$. In IL-13R $\alpha 1^{-/-}$mice granuloma expression of the eosinophil recruiting chemokine eotaxin (CCL11) was very much diminished, though there were normal levels of eosinophils in the granuloma. This may be accounted for by increased systemic IL-5 leading to greater expansion of eosinophils. IL-13R $\alpha 1^{-/-}$mice had slightly diminished eosinophil responses in a Schistosoma egg antigen (SEA) model of AHR. ${ }^{190}$

Two papers published in 1998 demonstrated IL-13 is critically important to the pathology associated with allergic airways models. The neutralisation of IL-13 activity by a fusion protein combining portions of IgG-Fc and the soluble IL13R $\alpha 2$ chain, lead to diminished acylcholine induced AHR in antigen sensitised and challenged mice ${ }^{191}$. Treatment of mice with IL-13 was also able to increase AHR, serum IgE levels and cause transient eosinophilia. This verified IL-13 was both necessary and sufficient for inducing AHR ${ }^{192}$. Further work comparing IL-4-/ and IL-4R $\alpha^{-/-}$animals demonstrated that AHR could be induced independently of IL-4191, 193. Adoptive transfer of antigen 
specific IL-13 sufficient CD4 ${ }^{+} \mathrm{T}$ cells but not IL-13-/- CD4 ${ }^{+}$T cells could induce AHR, despite detection of high levels of other Th2 associated outcomes, including IL-4 and IgE production, driven by the IL-13 deficient cells ${ }^{194}$.

IL-13 is critical for the production of mucus in a range of Type II inflammatory models. In the lung non-ciliated cells of the airway epithelium, called Clara cells, have been shown to respond through the type II receptor causing them to become mucus secreting goblet cells in a process known as mucus metaplasia. Specific inhibition of IL-4R $\alpha$ on Clara cells alone lead to significant diminishment of mucus production in an allergic airways model, with decreases in the mucins Muc5ac, and Muc5b, and also the chitinase AmCase. Although there was no effect on AHR or other Type 2 associated inflammatory responses. ${ }^{195}$ Mucus production is another function that has been specifically attributed to IL-13, with the inhibition of IL-13 totally abrogating goblet cell hyperplasia and mucus production in the lung. ${ }^{192}$.

Mucus production is rare in the lung of healthy individuals, however goblet cells in the intestines are constitutively present and producing mucus. Increased intestinal mucus and the number of goblet cells is measured during infection with enteric parasites and these increases can be attributed at least in part to $\mathrm{T}$ cells ${ }^{196}$. Gastrointestinal parasites have been widely utilised to elucidate the roles of signalling through the IL-4R $\alpha$ chain in the intestines. Clearing lumen residing parasites usually involves a mechanism that increases expulsion; the mechanism that causes this is known as 'weep and sweep'188. For expulsion IL-4R $\alpha$ signalling is required on non-haematopoietic cells but not on haematopoietic cells, indicating that the mediators of the STAT6 dependent expulsive mechanism are intestinal stromal cells ${ }^{197}$. Clearance of intestinal infection is reliant on IL-13 ${ }^{198}$. Intestinal mucus is proposed to trap the parasites in the lumen, inhibiting their attachment to the gut wall and potentially affecting their feeding, this is what is referred to in the 'weep' of the weep and sweep mechanism ${ }^{188}$. The sweep refers to the increased rate of peristalsis observed in parasite resistant intestines, this occurs via increased smooth muscle contractility. Both IL-4 and IL-13 cause hyper-contractility of smooth muscle cells but IL-13 has a much greater effect ${ }^{199}$, 200. Treatment with IL-13 but not IL-4 caused a STAT6 dependent modification of smooth muscle response to the neurotransmitters acetylcholine and substance P. These IL-13 driven changes were shown to involve nerve signalling, as addition of the neurotoxin tetradotoxin diminished the hyper-contractility displayed by IL-13 treated smooth muscle. ${ }^{200}$ Therefore, the 
'weep and sweep' mechanism incorporates the production of parasite trapping mucus, increased luminal fluid and increased peristalsis to expel intestinal helminths in a STAT6 and mostly IL-13 dependent way.

Physiological changes in tissues are often observed where there are concentrated Type 2 immune responses ongoing, this is seen in the asthmatic lung ${ }^{142}$, helminth infected lung ${ }^{153}$, intestinal wall and liver during infection ${ }^{17}$, and in the skin during allergic dermatitis ${ }^{151}$. Hyper-proliferation of fibroblasts leading to increased collagen production and extracellular matrix (ECM) deposition is known as fibrosis. Excessive fibrosis disrupts organ architecture and eventually decreases function, significantly affecting an individual's quality of life. ${ }^{201}$ Both IL-4 and IL-13 can induce increased ECM production by fibroblasts in vitro ${ }^{202}$, but treatment with IL-13 neutralising antibodies has a greater effect on limiting pulmonary fibrosis than blocking IL-4 in fungal ${ }^{203}$ and FITC induced asthma models ${ }^{204}$. A similar pattern was observed when comparing the liver fibrosis induced by Schistosoma mansoni infection ${ }^{156}$. Fallon et al. demonstrated that Type 2 immune responses are required to control Schistosoma mansoni infection, as evidenced by the increased mortality observed in IL-4-/IL-13/- double knock-out mice. Intriguingly IL-13R $\alpha 1^{-/-}$mice not only had increased survival over WT mice, they also had decreased fibrosis, liver damage and obstructed biliary ducts. This data indicates that IL-4 is able to drive protective Type 2 immune responses. In contrast IL-13 is dispensable for controlling the infection and is also is one of the main causes of the fibrotic pathology associated with Schistosoma mansoni infection. ${ }^{156}$ Skin specific over expression of IL-13 also causes significant fibrosis and atopic dermatitis ${ }^{205}$. Furthermore, intradermal injections of IL-33 causes cutaneous fibrosis in an IL-13 dependent manner, indicating IL-13 signalling may be important in skin changes particularly in atopic dermatological diseases ${ }^{206}$. While it appears that IL-13 can modulate fibroblast function directly there is evidence it may be working by increasing the release of TGF- $\beta$ which effects stromal cell functions and proliferation ${ }^{207}$.

Both IL-4 and IL-13 are able to signal through the type II receptor but genetic knockout and antibody neutralisation experiments have shown that IL-13 is more important than IL-4 for driving many of the non-haematopoietic cell responses. This may be due to differential expression of receptor subunits, with very high expression of IL-13R $\alpha 1$, or very low expression of IL-13R $\alpha 2$ potentially increasing a cells ability to respond to IL-13. It is also possible that haematopoietic cells expressing the $\gamma_{c}$ can bind and 
respond to IL-4 with increased efficiency, essentially lowering the available IL-4 concentration. In either situation IL-13 is extremely important for modulating tissue responses during Type 2 inflammation, while IL-4 appears to have a greater effect upon immune cell activation and effector function.

\subsubsection{Intracellular controls on the expression of IL-4 and IL-13}

It remains uncertain which extracellular signals drive Th2 differentiation in vivo, however the intracellular events that mediate the switch of a newly activated $\mathrm{CD} 4^{+} \mathrm{T}$ cell into a fully functional Th2 cell have been extensively studied. The $I l 4$ promoter has many binding sites for mediators downstream of TCR and CD28 signalling, including NFAT, AP-1 and c-Maf, which promote IL-4 expression ${ }^{208}$. However some pathways initiated by TCR signalling are distinctly inhibitory, such as those involving the MAPK family member Erk and Src family kinases ${ }^{50}$. While IL-4 expression is one of the characterising features of Th2 cells there are several Th2 specific pathways that must be initiated for it to be expressed.

GATA3 is the master transcription factor for Th2 cells, and is critical for their effector functions. Enforced expression of GATA3 will drive Th2 differentiation even in Th1 polarising conditions ${ }^{209}, 210$, while deletion of the GATA3 gene inhibits the induction of Th2 cytokines IL-4, IL-5 or IL-13 in newly activated cells ${ }^{211}$. IL-4 signalling through STAT6 induces large increases in the expression of GATA3 in $\mathrm{CD}^{+} \mathrm{T}$ cells $^{212}$, although IL-4 independent expression can be induced by exposure to low concentrations of antigen ${ }^{213}$. Once expressed GATA3 has multiple roles in inducing Th2 cytokine expression and inhibiting signals that could promote an alternate phenotype, especially Th1. The DNA segment that contains the Il4, Il13 and Il5 genes is known as the Th2 locus. It contains multiple regulatory sites many of which are affected by GATA3214. The Il4 gene has several binding sites for GATA3, many associated with DNase I hypersensitive (HS) sites ${ }^{113}$. The Il4 gene's HSII site is located within intron 2, and binds GATA3. This site is critical for the optimal expression of IL4, however disruption of this site has no effect on IL-13 or IL-5 expression. Furthermore there is strong GATA3 dependent H3K4 trimethylation at the HSII site only in Th2 cells (not Th1), indicating that GATA3 may play a role in mediating epigenetic changes of the Th2 locus to stabilise Th2 differentiation. ${ }^{215}$ Binding of GATA3, along with NFAT1, to the HSVa site, located downstream from the Il4 gene, is also critical for normal expression of IL-4216, 217. The conserved GATA response element (CGRE) is 
located upstream of the $I l 13$ promoter and contains four sites that GATA3 can bind ${ }^{218}$, disruption of this region leads to diminished IL-13 expression while IL-4 and IL-5 remained unaffected ${ }^{219}$. The Th2 locus has a locus control region (LCR) positioned within the Rad50 gene, upstream of both $I l 13$ and Il4 ${ }^{214}$. GATA3 has multiple binding sites within the LCR and disruption of these sites causes decreases in either IL-4 expression alone, or expression of all three Th2 cytokines, depending on which binding site is deleted ${ }^{220}$. Finally, it has been reported that GATA3 may bind directly to the $I l 13$ and $I l 5$ promoter regions, which may explain why conditional knock-out of GATA3 in established Th2 cells results in loss of IL-13 and IL-5 expression, but IL-4 remains unaffected ${ }^{84}$.

Phosphorylated STAT5 binds several regulatory elements within the Th2 locus, of note the HSII and HSIII regions of the Il4 gene. It has been shown that expression of an activated form of STAT5a leads to IL-4 expression even without increasing GATA3 levels, although GATA3 is still required for this expression. Similarly even in high levels of GATA3 IL-4 cannot be expressed without STAT5 signalling. ${ }^{221}$ STAT5 is part of the intracellular signalling pathways of several cytokines, but for Th2 cells, IL-2 receptor signalling is essential for optimal Th2 differentiation ${ }^{222}$. It is proposed that STAT5 is important in maintaining the accessibility to the $I l 4$ gene, particularly during early differentiation before epigenetic modifications can occur to more permanently stabilise the locus. STAT5 can also promote GATA3 transcription ${ }^{223}$. As with GATA3 expression STAT5 signalling via the IL-2R is optimal in cells exposed to low antigen doses. Cells responding to high doses not only failed to express GATA3 but also had decreased responses to IL-2, inhibition of the MAPK pathway could release the inhibition on GATA3 and STAT5 signalling 223.

\subsubsection{Probabilistic regulation of IL-4 expression}

Between $0.5 \%$ and $15 \%$ of mammalian genes display random mono-allelic regulation where expression of the gene by different cells is randomly limited to one of the parental alleles. There are several mechanisms that mediate the regulation of allelic expression. Mammalian female cells inactivate one whole $\mathrm{X}$ chromosome, packaging them into Barr bodies, to ensure normal levels of transcription of $\mathrm{X}$ linked genes, and the chromosome a cell will express is set during early embryogenesis. Epigenetic imprinting in gametes can silence a gene from one parent while ensuring the gene from the other parent is the exclusively expressed allele. Lymphocytes also undergo a process called 
allelic exclusion to ensure they only produce a single form of antigen specific receptor. Expression of several cytokines by activated $\mathrm{CD} 4^{+} \mathrm{T}$ cells has been shown to be limited to a single allele, however which allele a cell expresses is not consistent across the population, and while some cells express mono-allelically others can express from both alleles (bi-allelically). ${ }^{224}$

The expression of both IL-4 and IL-13 is under allelic regulation in Th2 cells ${ }^{225}$, however the mechanisms controlling this are not fully understood. One explanation is that limited transcriptional machinery results in stochastic periods of expression from each of the alleles, so examination of a population at a given time point will find cells that are expressing mono-allelically and small population in a transition state expressing from both alleles. Another theory relies upon chromosomal alterations allowing stochastic availability of one allele or the other, and in a rare population of cells both alleles simultaneously. Positive feedback such as autocrine signalling and expression of transcription factors, GATA3 in the case of IL-4 and IL-13, can stabilise the remodelled chromatin allowing stable expression from the available allele $(\mathrm{s})^{212}$, at least until cell division when chromatin is repackaged. If the theory of chromatin remodelling is true it may be expected that stabilisation of one section of chromatin would ensure the expression of both IL-4 and IL-13 from the same chromosome, however it has been observed that IL-4 and IL-13 are regulated separately ${ }^{226}$. Cells expressing IL-4 monoallelically do not show a preference for expression of the IL-13 allele on the same chromosome. The possibility that chromatin remodelling occurs independently for each of the adjacent genes cannot yet be dismissed, so allelic availability mediated by chromatin changes remains a viable potential model for allelic regulation.

The mechanisms controlling mono-allelic and bi-allelically expressing cells have been postulated to play an important role in limiting the amount of cytokine produced by the total population ${ }^{169}$. Only a relatively minor population of polarised Th2 cells can be detected expressing IL-4 or IL-13 at any one time, despite all activated cells expressing equal levels of GATA $3^{227}$. It is proposed that mechanisms controlling allelic expression may actually be in place to regulate the number of cytokine producing cells, a more effective way of controlling cytokine levels then controlling the amount each individual cell releases ${ }^{169}$. 


\subsection{Group 2 innate lymphoid cells}

\subsubsection{The populations of innate lymphoid cells}

Recently several distinct innate cell populations have been characterised that are able to carry out some of the functions usually attributed to the adaptive lymphoid cells, despite the absence of antigen specific receptors. ILCs, residing in non-lymphoid tissues around the body respond to tissue-derived signals and contribute to inflammation by producing cytokines $^{15}$. ILCs are hematopoietic cells that express CD45 and Thyl (CD90) but distinctly lack expression of cell surface molecules that are associated with other immune cell lineages. Lineage markers that are commonly used to isolate ILCs include cell surface molecules found on; T cells CD3, CD4, CD8, B cells CD19, B220, granulocytes, Gr-1, CD11b, FceRI and the DGs CD11c 228 . However, the specific cocktail of lineage specific antibodies used varies from laboratory to laboratory, and this has caused some confusion especially in early publications as to what exactly defined an $\mathrm{ILC}^{15}$. All ILGs depend upon the transcription factor inhibitor of DNA binding 2 (Id2) for their development ${ }^{229}$. The ILC populations can be classified by both their expression of cell surface molecules and also expression of the master transcription factors often associated with the T helper subsets Th1, Th2, Th17 and Th22; T-bet, GATA3, Ror- $\gamma \mathrm{t}$ and aryl hydrocarbon receptor respectively ${ }^{229}$. Since 2010 there have been increasing numbers of publications implicating the different ILC populations in a range of inflammatory situations. 


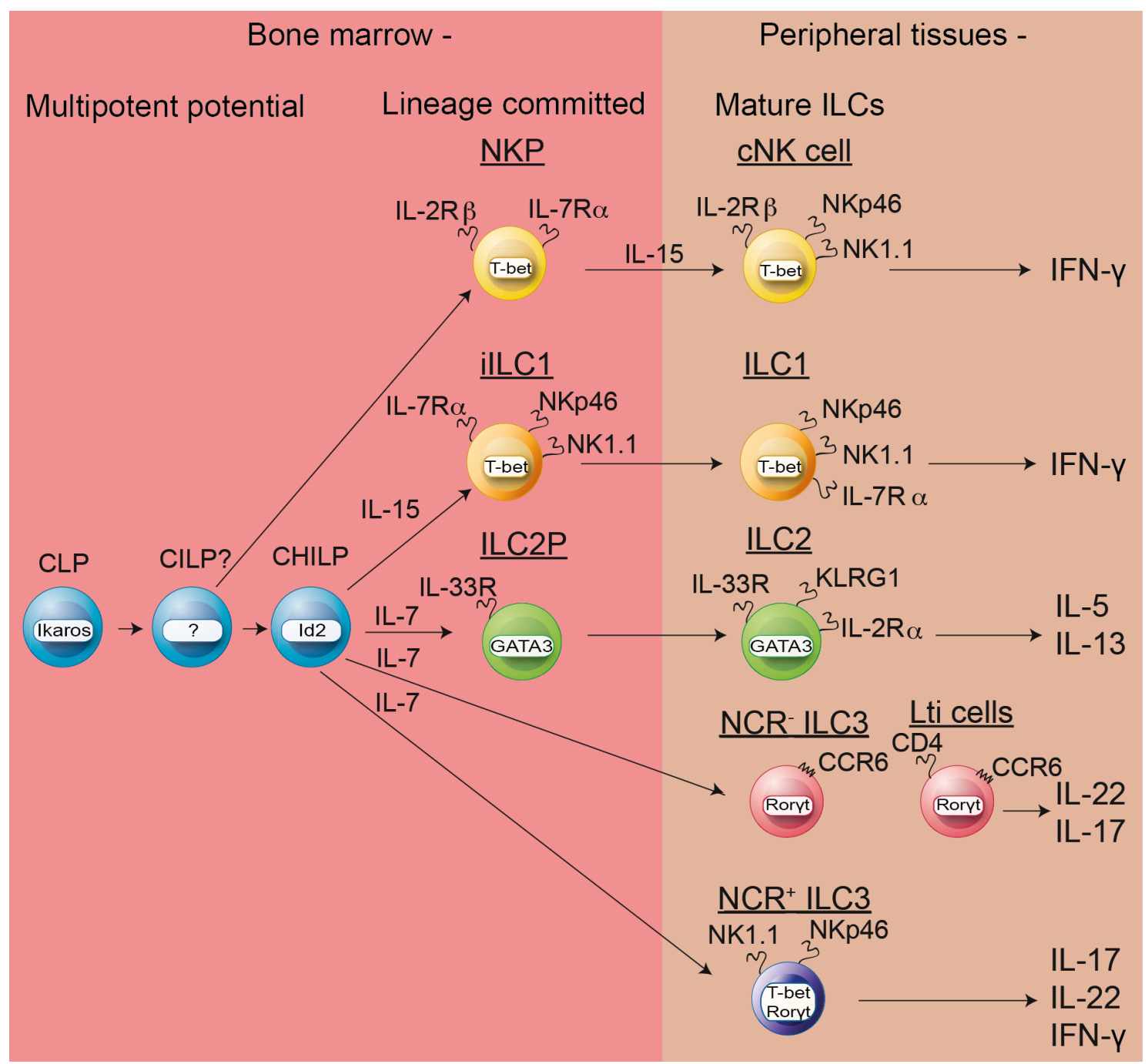

Figure 1.3 - Ontogeny of the innate lymphoid cell populations.

Common lymphoid progenitor (GLP) cells differentiate into an Id2 expressing population of common helper-like ILG progenitor (GHILP) through a proposed common ILG (CILP) precursor. Natural killer (NK) precursor (NKP) derives from the CILP cells before becoming conventional NK (cNK) cells. IL-15 dependent immature ILC1 express T-bet in the bone marrow before becoming mature ILC1s in the periphery. GATA3 expressing ILC2 precursors depend on IL-7 and mature in the periphery. All three populations of ILC3s require IL-7 signalling, including the lymphoid tissue inducer (Lti) cells. Modified from Klose and Diefenbach, 2014230 . 
In early 2013, after 2 years of publications identifying and attributing roles to different populations of ILGs a naming and grouping system was proposed that would cover the lineages and functions of the identified ILG populations and standardise their nomenclature ${ }^{15}$. The different populations were gathered into three broad categories named group 1, group 2 and group3. Group 1 contained both NK cells, and the putative ILC1s, these populations were largely grouped based on their expression of IFN- $\gamma$. It was initially unclear if ILC1s represented a unique population due to their similarities with NK cells, however recent papers have identified them as distinct lineages $^{231}$. NK cells require Eomes for their development while ILC1s do not, ILC1s however are dependent on both T-bet expression for normal generation and the depletion of T-bet only marginally inhibits NK cell. Promyelocytic leukaemia zinc finger (PLZF) is a transcription factor that has been shown to preferentially drive ILC, rather than NK cell development from the common lymphoid progenitor stage ${ }^{232}$. As with NK cells, ILC1s rely upon IL-15 for their development and survival in tissues.

A relatively diverse range of different ILC populations are encompassed within the group 3 ILCs (ILC3s), their reliance upon the transcription factor Ror- $\gamma t$ is the common element which unites the populations ${ }^{233}$. Lymphoid tissue inducer (LTi) cells, as with NK cells, have been well studied before the discovery of other ILC populations, but retrospective comparisons have meant they have been amalgamated within the ILC3 group $^{15}$. LTi cells are required for the development of secondary lymphoid tissues and upon stimulation produce both IL-17 and IL-22234. Two other Ror- $\gamma$ t dependent ILC3 populations have been defined that are divergent from LTi cells, in mice they can be distinguished by their lack of CD4233. One population only expressed IL-22 not IL-17, and these cells express the NK cell associated natural cytotoxicity triggering receptor (NCR) NKp46, thus they have been named the $\mathrm{NCR}^{+}$ILC3s and express T-bet at low levels alongside Ror- $\gamma \mathrm{t}$ and the aryl hydrocarbon receptor ${ }^{235}$. The third ILC3 population, the NGR- ILC3s, do not express T-bet and can produce both IL-17 and IL$22^{236}$. These three populations are similar in many ways and there may be much plasticity between them. This is highlighted by the fact that T-bet deficient mice lack $\mathrm{NCR}^{+}$ILC3s, so it may simply be the relative expression of the mutually repressive Ror- $\gamma$ t and T-bet that differentiate the $\mathrm{NGR}^{+}$and NGR- ILC3 populations ${ }^{237}$.

The group 2 ILGs consist of only a single population, the ILC2s. These cells are critically dependent on GATA3 ${ }^{238}$ for their function and produce IL-5, IL-13239, 
amphiregulin ${ }^{240}$ and IL-9116 in response to tissue derived cytokines such as IL-33, IL$25^{239}$ and thymic stromal lymphopoietin (TSLP) ${ }^{241}$. As with the ILC1s and all ILC3 populations, ILC2s rely upon IL-7 for their development and express the IL-7R (CD127), however ILC2s also constitutively express the IL-2R $\alpha$ chain (CD25), ICOS and the IL-33R (T1-ST2) ${ }^{15}$. All of the ILC populations (excluding NK cells) require GATA3 for their development however only the ILC2s maintain the expression of GATA3 once mature ${ }^{242}$.

Functionally, and by expression of certain transcription factors, the different ILC populations share many characteristics with $\mathrm{T}$ cells effector phenotypes. Group 1 ILCs rely upon T-bet and produce IFN- $\gamma$, like Th1 cells ILC1s have been shown to respond to IL-12 and IL-18 and contribute to immune responses against intracellular pathogens ${ }^{243,244}$. Both in terms of function and the reliance on Eomes, parallels have also been drawn between NK cells and memory $\mathrm{CD}^{+} \mathrm{T}$ cells ${ }^{245}$. The group 3 ILCs mirror Th17 cells and have been shown to play roles in controlling pathogenic intestinal bacteria $^{246,247,248,249}$. All group 3 ILCs respond to IL-1 $\beta$ and IL-23, which are largely produced by innate immune cells ${ }^{250}$. The barrier derived cytokines IL-25, IL-33 and TSLP have been demonstrated to induce cytokine responses from Th2 cells, and have similar effects on ILC2s. Both Th2 and ILC2s rely on GATA3 for their maintenance and function. Largely through their production of IL-5 and IL-13, ILC2s have been implicated in many models of Type 2 inflammation; from helminth infections to asthma and allergic inflammation of the skin ILC2s appear to be able to recapitulate many of the functions of peripheral tissue Th2 cells. ${ }^{251}$

\subsubsection{Identification and functions of group 2 ILCs}

ILC2s were not identified as a unique population until 2010, however earlier work had indicated Th2 associated cytokines could be elicited from a non $\mathrm{T}$ cell population by stimulation with IL-25. IL-25 was found to increase the expression of IL-5 and IL-13 from a population of non-B non-T cells, and this was enough to induce expedited expulsion of $\mathcal{N}$. brasiliensis from the gut ${ }^{252}$. In 2010 four papers were published independently identifying populations of what appeared to be closely related cell types in fat associated lymphoid clusters ${ }^{253}$, intestines ${ }^{254,255}$ and many other tissues ${ }^{256}$. These cells could all respond to IL-25 and/or IL-33 and produce Type 2 cytokines. Retrospective review found that while three papers had been focused on phenotypically 
similar ILCs, the paper by Saenz et al had identified another population of cells that did not express Thyl (CD90), IL-7R or T1.ST2 but did display abilities of multipotent progenitors (MPPs). Stimulation of these cells generated monocytes/macrophages, basophils and mast cells. This progenitor ability is not shared by any of the ILCs, showing that MPPs and ILCs are distinct populations of cells. ${ }^{239}$

c-Kit was originally used as an identifying molecule for ILC2s, however IL-13 reporter mice demonstrated that only a proportion of non-B non-T cells reporting IL-13 were c$\mathrm{Kit}^{+257}$. ILC2 populations in multiple tissue sites consistently express CD25, T1.ST2, CD69, CD44 and ICOS, further cementing their similarities with activated Th2 cells. Low levels of MHC II expression can be detected on ILC2s as well, indicating the potential for ILC2 - CD4+ T cell interactions. ${ }^{251}$

All ILC populations derive from CLPs arising from the bone marrow, expression of the transcription factor Id2 drives ILG, as opposed to $\mathrm{T}$ cell, NK cell or B cell, development ${ }^{258}$. The transcriptional regulators GATA3, retinoic acid receptor $\alpha$ $(\text { Ror } \alpha)^{259}$, T cell factor 1 (TCF-1)260 and growth factor independent 1 (Gfil) $)^{261}$ are all critical mediators at various steps of ILC2 development. GATA3 is lowly expressed by cells that maintain the potential to become all three ILC populations, and it's expression level increases as cells become ILC2 precursors (ILC2Ps) and eventually mature ILC2 $\mathrm{s}^{242}$. ILC2Ps are found in the bone marrow and in some tissues. They have diminished cytokine producing abilities compared with mature ILC2s, and can be distinguished by their decreased expression of killer cell lectin-like receptor subfamily $\mathrm{G}$ member 1 (KLRG1) ${ }^{238}$. GATA3 may play a role in the stabilization of Gfi expression ${ }^{262}$, while Gfil and TCF-1 both promote GATA3 potentially indicating a reinforcement pathway for ILC2 development ${ }^{251}$. Gfil increases expression of both IL25R and T1.ST2261, while TCF-1 promotes IL-7R and CD25263, some of the key receptors required for ILC2 maintenance and activation. Ror $\alpha$ deficient mice lack mature ILC2s, and the few ILC2P that can be found are negative for T1.ST2. Notch signalling and cytokine dependent stimulation of CLPs from the thymus can generate ILC2s, however athymic mice have peripheral ILC2s indicating that the thymus is not critical to ILC2 development ${ }^{259}$.

GATA3 stabilizes the Th2 locus in an open position promoting cytokine expression ${ }^{264}$. The high level of GATA3 expression in ILC2s is likely required for their ubiquitous IL13 and IL-5 production as demonstrated using reporter mice ${ }^{265,266}$. GATA3 also opens 
the IL-4 locus, which explains why IL-4 expression by mRNA and fluorescent reporter can be detected from ILGs but only with stimulation that induces calcium flux such as ionomycin and phorbol 12-myristate 13-acetate (PMA) stimulation ${ }^{251}$. This mirrors what has been seen with differentiated Th2 cells, where NFKB signalling pathways (such as those induced by IL-33 signalling) promote IL-5 and IL-13 expression ${ }^{267}$ while IL-4 expression requires calcium signalling and NFAT, which in Th2 cells is provided by the TCR $^{268,269 .}$

Through their production of IL-13 ILC2s play an important role in the gut for the expulsion of intestinal helminths. Administration of IL-33 or IL-25 induces strong Type 2 cytokine responses even in $\mathrm{T}$ cell deficient $\mathrm{Rag} 2^{-/-}$mice, and the responding cells were found to be ILC2 $2 \mathrm{~s}^{254,256}$. Adoptive transfer of WT ILC2s into IL-25R ${ }^{-/-}$mice was able to rescue exogenous IL-25 driven expulsion of $\mathcal{N}$. brasiliensis, however, this expulsion is depended upon IL-13 expression ${ }^{254}$. Transfer of ILC2s, along with IL-25 can similarly rescue normal $\mathcal{N}$. brasiliensis expulsive responses in $\gamma^{-/-R a g 2^{-/-}}$mice ${ }^{256}$. While Rag2-/mice have increased numbers of ILC2s, likely due to the decreased competition from T

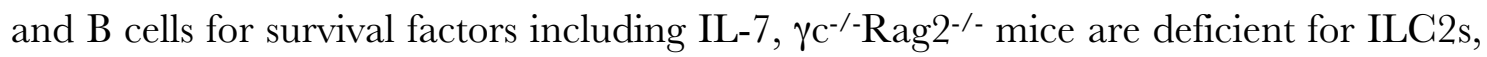
as the $\gamma_{\mathrm{c}}$ is required for IL-7 signalling 251 . IL-5 production by ILC2s supports IgA production and survival of $\mathrm{B} 1$ cells within the fat associated lymphoid clusters of the mesenteries ${ }^{253}$. An interesting model of micronutrient deficiency demonstrated that in the absence of vitamin A ILC2s expanded at the cost of ILC3 populations within the intestine. Expanded ILC2s lead to increased expulsion of helminths, potentially linking nutrient deficiency to protective responses against parasites, however the decreased ILC3 populations also left the mice susceptible to bacterial invasion. ${ }^{270}$

In the lung ILC2s have been demonstrated to play roles in both Type 1 and Type 2 associated immune responses ${ }^{240}$. While in the intestines both ILC2 and ILC3 populations can be identified, the lung has been characterised as being dominated by group 2 innate lymphoid cells ${ }^{270}$. IL-33271 and IL-25257 can both induce lung ILC2 expansion, cytokine expression and AHR, but IL-33 induces more resilient responses than IL-25272. Again, adoptive transfer of cytokine stimulated WT ILC2s was sufficient to induce AHR in IL-13-/ mice in an IL-13 dependent manner ${ }^{257}$. ILC2s are major producers of Type 2 cytokines in AHR models induced by the administration of $\mathrm{OVA}^{257}, \mathrm{HDM}^{273}$, papain protease ${ }^{274}$ or Alternaria alternata allergen ${ }^{271}$. IL-33 was critical to AHR and ILC2 responses in OVA and ragweed induced airways inflammation ${ }^{271}$, 
while HDM induced AHR demonstrated a greater reliance on IL-25273. ILC2s were shown to be major producers of IL-9 in the lung in responses to papain. This IL-9 signalled in an autocrine fashion increasing IL-5 and IL-13 production, and was found to increase the survival of pulmonary ILC2s during infection by $\mathcal{N}$. brasiliensis $^{74}$. ILC2s also play an important role in lung repair, mice depleted of ILC2s had decreased survival after infection with Type 1 immune response inducing influenza. Amphiregulin, a member of the epidermal growth factor family, is produced by ILC2s and mediates repair of the lung epithelia damaged by influenza infection. Depletion of ILC2s decreased these repair responses and increased the chance of death from the infection. ${ }^{240}$

Increasing evidence indicates that ILC2s play critical roles in maintaining fat and metabolic homeostasis. IL-5 $5^{-/-}$mice are more susceptible to obesity when on a high fat diet while transgenic IL-5 overexpressing mice are leaner and have increased glucose tolerance 275,276 . This along with the fact that IL-4 administration protects from insulin resistance on a high fat diet ${ }^{277,278}$ indicates that Type 2 immune response may not be evolutionarily conserved simply for protection against multicellular parasites. ILC2s maintain alternately activated macrophages and eosinophils within visceral adipose tissues $^{275}$. Eosinophil numbers in visceral adipose tissues fluctuate in a circadian rhythm that is dependent upon IL-5 production by ILCs responding to neuropeptides released upon feeding 279 . Alternately activated macrophages sequester iron and inhibit its accumulation in adipocytes, which can induce the production of lipid aldehydes and effect adipocyte function ${ }^{280}$. Through the production of IL-13 and the recruitment of IL-4 producing eosinophils, ILC2s control alternately activated macrophages in subcutaneous fat which are responsible for the conversion of white adipose cells to thermogenic beige adipose cells in response to cold stress. IL-4 and IL-13 also expand a population of adipocyte precursor cells that are predisposed to becoming beige adipocytes. $^{281}$ ILC2s not only have roles in expelling helminths from the gut and causing asthmatic responses in the lung, they also maintain health through epithelial repair mechanisms and controlling of obesity by maintaining homeostatic conditions within fat tissues. 


\subsubsection{Reporter mice for identification of cytokine producing cells; the benefits and caveats}

Genetically modified mice have become an invaluable tool for studying the biology of the immune system, both at homeostasis and in disease settings. Early genetic engineering techniques developed by Gordon, Plotkin, Barbosa and Ruddle in 1980 simply involved microinjection of genetic material into the embryos of mice, these DNA segments would randomly insert into the genome. As long as the embryo was freshly fertilized and hadn't undergone cell division when the DNA was injected, all the cells of the mature mouse would carry the genetic material. This technique created the first transgenic mice. ${ }^{282}$ While this technology was effective for introducing genetic material into a mouse strain, it was imprecise; parental genes could not be targeted and implantation could disrupt expression of unintended genes.

The development of targeted mutagenesis allowed disruption of desired genes and insertion of genetic material that can be controlled by the promoter of a gene of interest. ${ }^{283}$ This technique relies upon homologous recombination, whereby similar double stranded DNA can be swapped via one of several mechanisms. Eukaryote cells use this to repair double stranded breaks and for the swapping of genetic material between homologous chromosomes during the generation of gametes. Addition of a section of DNA to a cell with 5' and 3' sequences that match germ line DNA sequences can lead to integration of the novel DNA section into the germ line via homologous recombination. ${ }^{284}$ These recombination events are rare so selection strategies are required to identify cells that have successfully incorporated the foreign DNA. The most common technique is the insertion of a gene that allows antibiotic resistance, allowing selection by culturing of cells in antibiotic spiked media, thus only cells that have incorporated and are expressing the DNA construct containing the resistance gene will survive. To generate genetically modified mice using this technique embryonic stem (ES) cells are microinjected with a DNA construct that includes the sequence of an antibiotic resistant gene and 3' and 5' sequences that are homologous for sections of a target gene. Successful incorporation of the construct can be selected for by identifying surviving ES cells cultured in antibiotic media. These modified ES cells are transferred into a mouse blastocyst and this is implanted into a pseudo-pregnant female to eventually give rise to chimeric offspring that have a proportion of cells heterozygous for the targeted gene. These mice can be bred and their offspring tested by PCR for the presence of the modified gene. Finally, the antibiotic resistance sequence can be spliced 
by utilising Cre-Lox technology, crossing these mice to a strain that constitutively expresses the Cre recombinase enzyme can lead to the splicing out of the antibiotic resistant gene if it was originally designed with Lox-P sites on either side of the sequence. ${ }^{285}$

If the 3' and 5' sequences of the inserted DNA construct are spaced across exons of a targeted gene, these exons are excised during the recombination event, disrupting the sequence of the gene and leaving the cell unable to express protein from that allele. Crossing of these mice can generate offspring homozygous for the disrupted allele (assuming that the targeted gene is not essential for development or survival of the mouse). This allowed the generation of knock-out mice, mice that could not express RNA or protein from a gene of interest. ${ }^{284}$ Using knock-out mice allowed study of the roles particular genes play in modulating immune responses, for instance IL-4 knockout mice have been used to demonstrate that IL-4 expression increased susceptibility to Leishmania major infection. ${ }^{176}$

Addition of sequences that produce functional proteins into an inserted DNA construct, other than those that conveyed antibiotic resistance, increased the scope of homologous recombination based targeted gene modification. The insertion of genes that produce fluorescent proteins allows identification of cells that are actively transcribing the modified gene and this is how knock-in fluorescent reporter mice were created. Reporter mice are valuable for characterising the cells that are producing soluble proteins, such as cytokines. Their identification would generally require restimulation in the presence of poisons that inhibit golgi body function followed by fixation and intracellular antibody staining or western blotting. Knock-in reporter constructs can allow identification of cells that are transcribing the protein of interest in real time and directly ex-vivo without any further manipulation. ${ }^{286}$

Several different variations of IL-4 reporter mice have been generated each with different systems for marking IL-4 producing cells. Two types of IL-4 green fluorescent protein (GFP) reporter mice have been produced, one known as the G4 mouse that is a simple knock-in reporter with the GFP gene replacing the first exon and 178 nucleotides of the first intron of the IL-4 gene ${ }^{227}$. The second GFP reporter designated IL- $\underline{4}$ / GFPenhanced transcript, or 4get mice employed an internal ribosome entry site (IRES) sequence in an attempt to avoid disruption to the parental IL-4 genes. The IRES-GFP sequences were targeted just downstream of the translational stop codon of the IL-4 gene, allowing the full transcription of the IL-4, IRES and GFP mRNA. This permitted 
normal translation of the IL-4 mRNA which would cease at the endogenous stop codon, however the IRES sequence allowed transcription of GFP despite it's sequence beginning part way through the mRNA strand. The 4get mice should therefore be able to produce normal IL-4 levels, but also report by GFP expression any cell that is transcribing IL-4 mRNA. 287

A third type of IL-4 reporter mouse was generated twice by two different groups on separate occasions, and is also a knock-in mouse with the modified sequence for the human CD2 (huCD2) molecule replacing just the first exon of the IL-4 gene. When the IL-4 promoter was engaged huCD2 would be expressed, and these molecules would be translocated to the cell surface. The normal intracellular signalling sequences had been modified so the huCD2 would be unable to signal within the mouse cells even if it was bound to a ligand. By staining with fluorescently bound antibodies specific for human CD2, cells that are producing IL-4 can be identified. ${ }^{288}$ The huCD2 and G4 GFP reporters are constructed similarly and appear to work in similar ways; only $\mathrm{T}$ cells that are actively expressing the IL-4 allele that carries the reporter are positive. The 4get mice are referred to as mRNA reporter mice, they mark a cell that is competent for IL4 expression but not necessarily a cell expressing IL-4 protein. For example, a Th2 cell that has been differentiated will remain reporter positive even after the cell ceases IL-4 protein production. ${ }^{289}$

In an attempt to retain protein sufficiency, but restrict reporting only to protein producing cells, recent reporters have been created using variants on older transgenic technology. To create transgenic reporters a bacterial artificial chromosome (BAC) carrying a section of mouse chromosome with the gene of interest is created or screened for from a library. Employing the same homologous recombination technology as used to create knock-in reporters; the reporter sequences are inserted after the promoters of the gene of interest within the BACs. The BAC vectors are then isolated, linearized and microinjected into embryonic stem cell for transfer into a blastocyst. These transgenic reporters have a third allele of the gene of interest that has been disrupted by the reporter construct inserted randomly into their genome. While these mice are able to produce normal protein from their parental alleles, the reporters being randomly inserted in the genome may be missing regulatory elements that enhance or control gene expression of the parental alleles, meaning their reporting may not consistently reflect parental gene expression. ${ }^{290}$ 
Reporter mice can allow temporal and spatial identification of protein producing populations of cells in a range of models however each method of reporter generation comes with caveats that need to be considered when interpreting data produced using these systems.

\subsection{Thesis Aims}

In this thesis I aim to investigate how cells regulate their cytokine production in a number of models of Type 2 inflammation. To do this I will validate and use a novel IL-4 and IL-13 reporter mouse. I will investigate how $\mathrm{CD}^{+} \mathrm{T}$ cells mediate their allelic regulation of IL-4 expression and compare how these cells and innate immune cells control IL-4 and IL-13 expression. The principle aim of this work is to understand if Type 2 cytokines are regulated in the same way or if there are refined mechanisms that differentially regulate the expression of these cytokines. The specific aims are:

- To assess how CD4 ${ }^{+} \mathrm{T}$ cells regulate allelic expression of IL-4 in vitro and how IL-4 contributes to the generation of Th2 cells in an in vivo model of Type 2 inflammation.

- To compare of CD4+ $4^{+}$cell expression of IL-4 and IL-13 and how Th2 cells regulate the production of IL-13 both in vitro and in vivo.

- To identify and characterise a dermal ILC population and determine how these cells, and basophils in the skin, produce cytokines in response to models of skin inflammation.

- To compare cytokine production by lung $\mathrm{CD} 4^{+} \mathrm{T}$ cells and ILC2s in response to infection by $\mathcal{N}$. brasiliensis and how these cells interact to maintain protective immune responses. 


\section{Materials and Methods}

\subsection{Materials}

\subsubsection{Labware}

\begin{tabular}{|c|c|}
\hline $\begin{array}{l}\text { Supplier/ } \\
\text { Manufacturer }\end{array}$ & uct \\
\hline $\begin{array}{l}\text { Alphatech Systems } \\
\text { Ltd \& Co, Auckland, } \\
\text { NZ. }\end{array}$ & $\begin{array}{l}\text { - Acrodisc } \AA \text { Serum Filter. Glass Fiber prefilter, 0.2um } \\
\text { Supor membrane, Sterile, } 37 \mathrm{~mm}\end{array}$ \\
\hline $\begin{array}{l}\text { Axygen Scientific } \\
\text { Inc., Union City, CA, } \\
\text { USA. }\end{array}$ & $\begin{array}{l}\bullet 0.5-20 u l, 200 u l, 1000 u l \text { Tips, Racked \& Pre-Sterilized } \\
\bullet 0.6 \mathrm{ml}, \quad 1.7 \mathrm{ml}, \quad 2.0 \mathrm{ml} \text { MAXYMum Recovery } \\
\text { Microtubes }\end{array}$ \\
\hline $\begin{array}{l}\text { Becton Dickinson Pty } \\
\text { Ltd, Mountain View, } \\
\text { CA, USA. }\end{array}$ & $\begin{array}{l}\text { - BD Ultra-Fine }{ }^{\mathrm{TM}} \text { \& Ultra-Fine }{ }^{\mathrm{TM}} \mathrm{II} \text { Insulin Syringes - } \\
\text { Bulk Packaged - Box/100 15ml, 50ml high-clarity } \\
\text { polypropylene conical centrifuge tube } \\
\text { - 5ml, 10ml, } 25 \mathrm{ml} \text { polystyrene serological pipet. } \\
\text { - } 12 \text { x75 mm, } 5 \mathrm{ml} \text { polystyrene round bottom test tube. } \\
\text { No cap. Non-sterile. } \\
\text { - BD Falcon }{ }^{\mathrm{TM}} 175 \mathrm{~cm}^{2} \text { Cell Culture Flask, } 750 \mathrm{ml} \text {, } \\
\text { tissue culture treated, straight neck, black lined } \\
\text { phenolic screw cap. } \\
\text { - BD Falcon }\end{array}$ \\
\hline
\end{tabular}




\begin{tabular}{|c|c|}
\hline & 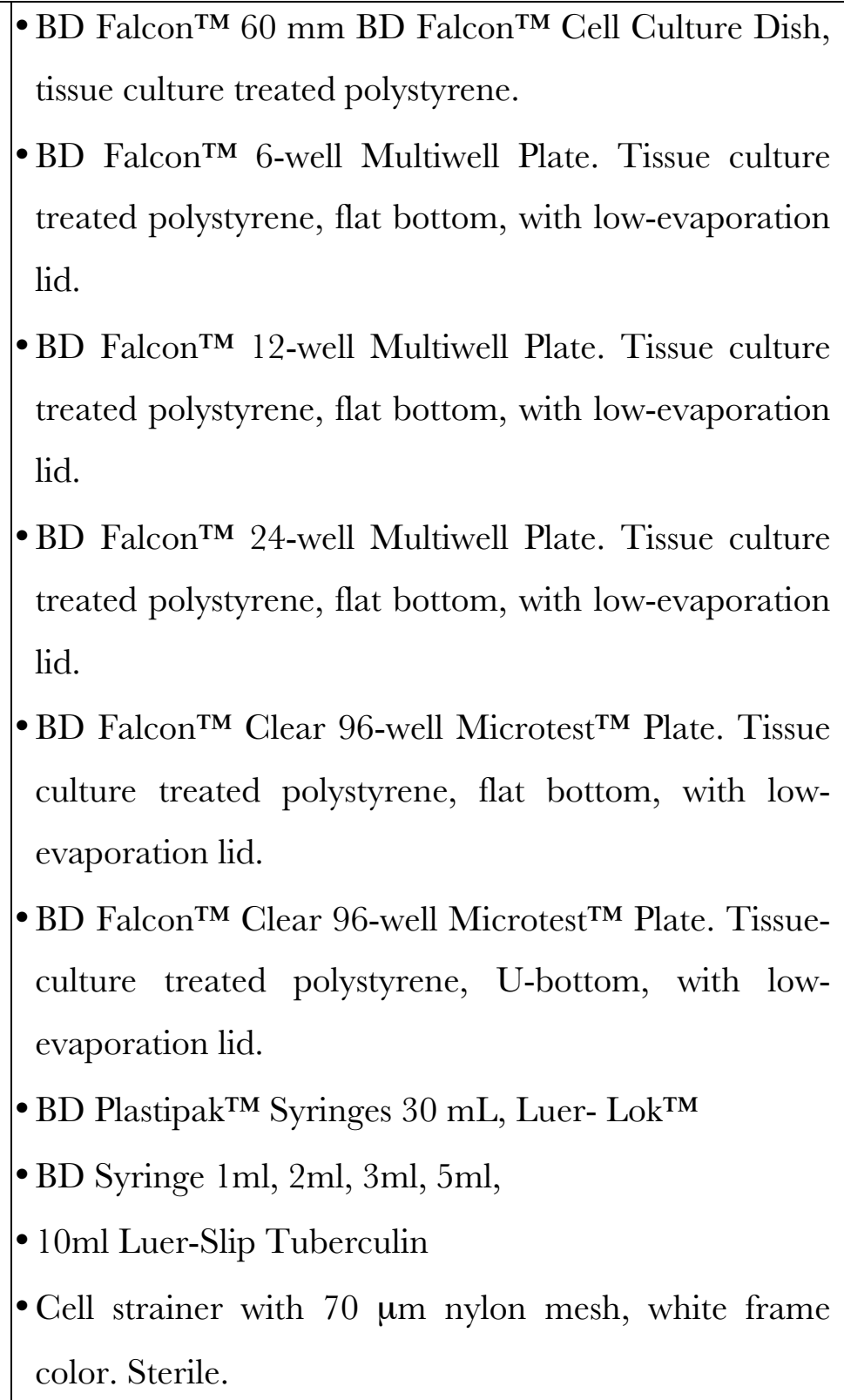 \\
\hline $\begin{array}{l}\text { GE Healthcare, } \\
\text { Auckland, New } \\
\text { Zealand }\end{array}$ & - HI Trap protein G columns \\
\hline $\begin{array}{l}\text { Hawksley Medical } \\
\text { Laboratory Products, } \\
\text { Lancing UK. }\end{array}$ & Improved Neubauer Haemocytometer \\
\hline $\begin{array}{l}\text { Miltenyi Biotec, } \\
\text { Bergisch, Germany. }\end{array}$ & AutoMACS Pro Separator Instrument \\
\hline $\begin{array}{l}\text { Molecular Devices, } \\
\text { Sunnyvale, CA, USA. }\end{array}$ & VersaMax ELISA Microplate reader \\
\hline Olympus & CX21 Compound Microscope \\
\hline
\end{tabular}




\begin{tabular}{|c|c|}
\hline $\begin{array}{l}\text { Corporation, } \\
\text { Shinjuku, Japan. }\end{array}$ & $\begin{array}{l}\text { CX40 Inverted Microscope } \\
\text { SZX10 Stereo Microscope }\end{array}$ \\
\hline $\begin{array}{l}\text { Sefar Filter Specialist, } \\
\text { Nelson, New Zealand. }\end{array}$ & $\begin{array}{l}\text { 70um nylon gauze } \\
80 \mu \mathrm{m} \text { stainless steel mesh }\end{array}$ \\
\hline $\begin{array}{l}\text { Thermo Fisher } \\
\text { Scientific, Waltham, } \\
\text { MA, USA. }\end{array}$ & $\begin{array}{l}\text { - Nunc CryoTube 1.0mL 1.8mL PP sterile } \\
\text { - Nunc-Immuno }{ }^{\mathrm{TM}} \text { Plates Polystyrene, without lids. } 96 \\
\text { wells per plate. MaxiSorp }{ }^{\circledR} \text {. } \\
\text { - Transfer pipette graduated } 1 \mathrm{ml} \text { large bulb } \\
\text { - SecureSeal }{ }^{\mathrm{TM}} \text { Thermal Adhesive Sealing Film for } \\
\text { PCR application } \\
\text { - Slide-A-Lyzer Dialysis Cassettes, } 10000 \text { MWCO } \\
\text { - Glass Pasteur pipette } 150 \mathrm{~mm}(\mathrm{p} / 1,000) \\
\text { - Sartorius Stedim Vivaspin } 20 \mathrm{ml} \text { tubes } 10000 \mathrm{MWCO} \\
\text { - Nanodrop } 1000 \text { Spectrophotometer }\end{array}$ \\
\hline
\end{tabular}




\subsubsection{Reagents and buffers}

\subsubsection{Culture media reagents}

2-mercaptoethanol

$2 \mathrm{ME}$ was from Invitrogen (Auckland, NZ, 21985-023) and stored in aliquots at $4^{\circ} \mathrm{C}$.

Penicillin-Streptomycin

Penicillin-Streptomycin was purchased in liquid form from Invitrogen (Gibco, Auckland, NZ, 15140-122) and stored as single use aliquots at $4^{\circ} \mathrm{C}$ until used.

Foetal bovine serum (FBS)

FBS was purchased from Invitrogen (GIBCO, Auckland, NZ, 12203C) and stored in 25 $\mathrm{ml}$ aliquots at $-20^{\circ} \mathrm{C}$.

Complete Iscove's modified Dulbecco's medium (cIMDM)

IMDM was purchased from Invitrogen (Auckland, NZ, 31980-097) and supplemented with $100 \mathrm{U} / \mathrm{ml}$ Penicillin-Streptomycin from Invitrogen (Gibco, Auckland, NZ, 15140122), $55 \mu \mathrm{M} 2 \mathrm{ME}$ and 5\% FBS. Media was filter sterilised and stored at $4^{\circ} \mathrm{C}$ for a maximum of 14 days.

\subsubsection{Enzymes and tissue processing reagents}

Dulbecco's phosphate buffered saline (dPBS)

dPBS powder containing no calcium or magnesium was purchased from Invitrogen (Gibco, Auckland, NZ, 21600-069) and dissolved in dH2O.

Collagenase I

Collagenase I was purchased from Invitrogen (Auckland, NZ, 17100-017). The lyophilised powder was reconstituted in cIMDM to a concentration of $2.4 \mathrm{mg} / \mathrm{ml}$ and stored in aliquots at $-20^{\circ} \mathrm{C}$.

Collagenase IV (Collagenase from histolyticum)

Collagenase IV was purchased from Sigma-Aldrich New Zealand Ltd (Auckland, NZ, C5138). The lyophilised powder was reconstituted in dPBS to a concentration of 50 $\mathrm{mg} / \mathrm{ml}$ and stored in aliquots at $-20^{\circ} \mathrm{C}$. 
Red blood cell lysis buffer

RBC Lysing Buffer was purchased from Sigma-Aldrich New Zealand Ltd (Auckland, NZ, R7757) and stored at room temperature.

DNase I

DNase I was purchased as a lyophilised powder from Roche Diagnostics New Zealand Ltd (Auckland, NZ, 10104159001) dissolved in cIMDM to a concentration of 10 $\mathrm{mg} / \mathrm{ml}$ and stored at $-20^{\circ} \mathrm{C}$.

Ethylenediaminetetraacetic acid (EDTA)

Diluted EDTA (0.5M) was purchased from Invitrogen (Auckland, NZ, 15575-020) and stored at room temperature until used.

Liberase TL

Lyophilized enzyme was purchased from Roche Diagnostics New Zealand Ltd, (Auckland, NZ, 5401020001) stored at $-20{ }^{\circ} \mathrm{C}$ for long term storage. Enzymes were resuspended in IMDM and agitated to reconstitute to a concentration of $1 \mathrm{mg} / \mathrm{mL}$. Reconstituted enzymes were used immediately or stored at $-20^{\circ} \mathrm{C}$ for up to three months.

Sodium Hydroxide ( $\mathrm{NaOH})$

$\mathrm{NaOH}$ was purchased from BDH Laboratory Supplies (Poole, UK). Stored at $5 \mathrm{M}$ at room temperature.

Hydrochloric acid ( $\mathrm{HCl}$ )

$\mathrm{HCl}$ was bought from Merck KGaA (Darmstadt, Germany) and stored at $11.65 \mathrm{M}$ at room temperature.

Tris

UltraPure ${ }^{\mathrm{TM}}$ Tris was diluted in $\mathrm{ddH}_{2} \mathrm{O}$ to a $1 \mathrm{M}$ concentration. 
Lung digestion buffer

Collagenase type I at a final concentration of $2.4 \mathrm{mg} / \mathrm{ml}$ from stock at $24 \mathrm{mg} / \mathrm{ml}$ and DNAse 1 at $120 \mu \mathrm{g} / \mathrm{ml}$ from a stock of $10 \mathrm{mg} / \mathrm{ml}$ were made up in cIMDM to the required volume.

\section{Skin digestion buffer}

Collagenase type IV at a final concentration of $5 \mathrm{mg} / \mathrm{ml}$ from stock at $50 \mathrm{mg} / \mathrm{ml}$ and DNAse 1 at $120 \mu \mathrm{g} / \mathrm{ml}$ from a stock of $10 \mathrm{mg} / \mathrm{ml}$ were made up in cIMDM to the required volume.

\section{Spleen digestion buffer}

Liberase at a final concentration of $0.125 \mathrm{mg} / \mathrm{ml}$ from stock at $1 \mathrm{mg} / \mathrm{ml}$ and DNAsel at $100 \mu \mathrm{g} / \mathrm{ml}$ from a stock of $10 \mathrm{mg} / \mathrm{ml}$ were made up in cIMDM to the required volume.

\section{Alsever's Solution}

20.5 g Dextrose (BDH Laboratory Supplies, Poole, England), 8 g sodium citrate (BDH Laboratory Supplies, Poole, England) and $4.2 \mathrm{~g} \mathrm{NaCl}$ (Sigma, Auckland, NZ) were dissolved in distilled $1000 \mathrm{ml}$ MQ H2O (MilliQ) and the $\mathrm{pH}$ was adjusted to 6.1 with 1 M citric acid (BDH Laboratory Supplies, Poole, England). Alsever's solution was stored at room temperature.

\section{$1.8 \% \mathrm{NaCl}$ solution}

1.8g NaCl from Sigma-Aldrich New Zealand, Ltd (Auckland, NZ) was diluted in $100 \mathrm{~mL} \mathrm{ddH} \mathrm{H}_{2} \mathrm{O}$.

Tail lysis buffer for genomic DNA

$\mathrm{NaOH}$ and EDTA were diluted to $25 \mathrm{mM}$ and $0.2 \mathrm{mM}$ respectively in $\mathrm{ddH}_{2} \mathrm{O}$ and $\mathrm{pH}$ was set to 12 .

\section{Tail lysis neutralisation buffer}

Tris-HCl was diluted in $\mathrm{ddH}_{2} \mathrm{O}$ to a concentration of $40 \mathrm{nM}$ and $\mathrm{pH}$ was set with $\mathrm{HCl}$ to a $\mathrm{pH}$ of 5 . 


\section{Isolation buffer}

2 mM EDTA, 0.5\% FBS and 0.012\% DNase I were made up in dPBS, filter sterilised and stored at $4^{\circ} \mathrm{C}$.

\section{CD4 Dynabeads isolation kits}

Dynabead CD4 isolation kits were purchased from Invitrogen (Auckland, NZ, 11145D). Kits were stored at $-4^{\circ} \mathrm{C}$.

\section{CD11c Microbead isolation kits}

CD11c Microbead isolation kits were purchased from Miltenyi Biotec (Bergisch Gladbach, Germany, 130-052-001). Kits were stored at $-4^{\circ} \mathrm{C}$.

\section{Trypan Blue}

Trypan Blue was purchased from Invitrogen (Auckland, NZ, 15250-061) and stored at room temperature.

\subsubsection{Cytokines}

Recombinant human interleukin-2

Recombinant rhIL-2 (Proleukin) from Novartis Vaccines \& Diagnostics was stored lyophilised at $4^{\circ} \mathrm{C}$. It was made up in IMDM at a concentration of $10^{5} \mathrm{U} / \mathrm{mL}$, aliquots stored at $-20^{\circ} \mathrm{C}$.

\section{Interleukin-4}

Supernatant of IL-4 producing Chinese hamster ovary cells was collected. IL-4 activity was compared via ELISA to standards originally calibrated using CT.4S cell line as described ${ }^{30} .1$ unit is approximately $0.5 p g$ of IL-4.

\section{Interleukin-33}

Interleukin-33 purchased from R\&D Systems (Minneapolis, Minnesota, USA, 3626ML-010) and was resuspended in dPBS to a concentration of $10 \mu \mathrm{g} / \mathrm{mL}$. Aliquots were stored frozen at $-20^{\circ} \mathrm{C}$. 
Thymic Stromal Lymphopoietin (TSLP)

TSLP was purchased from R\&D Systems (Minneapolis, Minnesota, USA, 555-TS-010), lyophilised TSLP was stored at $-20^{\circ} \mathrm{C}$. TSLP was reconstituted in dPBS to a concentration of $10 \mu \mathrm{g} / \mathrm{mL}$ and aliquots are stored at $-20^{\circ} \mathrm{C}$.

Complexed IL-2 (IL-2c)

Recombinant murine IL-2 was purchased from Peprotech (Rocky Hill, NJ, USA, 21212) stored lyophilised at $-20^{\circ} \mathrm{C}$ and reconstituted to $1 \mathrm{mg} / \mathrm{ml}$ in dPBS. aIL-2 (clone JESS6-1 from WEHI, Melbourne, Victoria, AUS) was stored at shipping concentration at $-70^{\circ} \mathrm{C}$ and diluted to $1 \mathrm{mg} / \mathrm{mL}$ in dPBS. IL-2 and $\alpha$ IL-2 antibody was mixed at a molar ratio of $1: 5$ and incubated for 30 minutes at $37^{\circ} \mathrm{C}$. Aliquots of this mixture were stored at $-70^{\mathrm{a}} \mathrm{C}$. Prior to use these aliquots were diluted 1:16.67 in dPBS.

\subsubsection{Purified antibodies}

Rat anti-mouse CD3 (Clone 2C11)

Anti-mouse CD3 was affinity-purified in house from hybridoma culture supernatants using Hi Trap protein G-Sepharose columns.

Hamster anti-mouse CD28 (Clone 37.51)

Anti-mouse CD28 was grown in house from hybridoma culture supernatants were tested against previous supernatants in T cell cultures.

Rat anti-mouse IL-4 (Clone 11B11)

Anti-mouse IL-4 was affinity-purified in house from hybridoma culture supernatants using Hi Trap protein G-Sepharose columns.

Rat anti-mouse IFN- $\gamma($ Clone $A \mathcal{N}-18)$

Anti-mouse IFN- $\gamma$ was affinity-purified in house from hybridoma culture supernatants using Hi Trap protein G-Sepharose columns.

Rat anti-mouse MHC II (Clone M5/114.15.2)

Anti-mouse MHC II was affinity-purified in house from hybridoma culture supernatants using Hi Trap protein G-Sepharose columns. 
Rat anti-mouse IL-2 (Clone FES6-1A12)

Anti-mouse IL-2 was purchased from WEHI Antibody Services (Melbourne, Victoria, AUS). It was stored at shipping concentration at $-70^{\circ} \mathrm{C}$.

Rat anti-mouse CD4 (Clone GK1.5)

Anti-mouse CD4 was purchased from Bio X cell (West Lebanon, New Hampshire, USA). It was stored at shipping concentration at $-70^{\circ} \mathrm{C}$.

Rat anti-mouse CD90 (Clone 30H12)

Anti-mouse CD90 was purchased from Bio X cell (West Lebanon, New Hampshire, USA). It was stored at shipping concentration at $-70^{\circ} \mathrm{C}$.

Rat anti-mouse CD16 (FceRIII)/CD32 (FceRII) (Clone 2.4G2)

Anti-mouse CD16 (FceRIII)/CD32 (FceRII) was affinity-purified in house from hybridoma culture supernatants using Hi Trap protein G-Sepharose columns.

Rat anti-mouse IL-13

Anti-mouse IL-13 antibody was kindly provided by Abbvie. It was stored at $-70^{\circ} \mathrm{C}$ at shipping concentration.

\subsubsection{Allergens and peptides}

ISQ peptide from ovalbumin

Ova peptide ISQAVHAAHAEINEAGR (OVA323-339) was purchased from Mimotopes Pty Ltd (Clayton, VA, AUS) and referred to as ISQ peptide. Lyophilized protein was reconstituted in $\mathrm{dPBS}$ to $1 \mathrm{mM}$ and stored at $-80 \mathrm{oC}$.

\section{House dust mite}

House dust mite (HDM) purchased from Greer laboratories (Lenoir, NC, USA) was resuspended in sterile $\mathrm{dPBS}$ to $100 \mu \mathrm{g} / 30 \mu \mathrm{l}$ and stored at $-80^{\circ} \mathrm{C}$. 


\subsubsection{Drugs}

Anaesthetic

10x stock solution was diluted in sterile dPBS to $8.6 \mathrm{mg} / \mathrm{ml}$ ketamine and $0.26 \mathrm{mg} / \mathrm{ml}$ xylazine working solution.

\section{Calcipotriol (MC903)}

Calcipotriol was purchased from Cayman Chemicals (Ann Arbor, Michigan, USA). Calcipotriol was diluted in absolute ethanol from Pure Science (Porirua, New Zealand) to a concentration of $1.6 \mathrm{mM}$ and stored at $-20^{\circ} \mathrm{C}$. Diluting stock solution 1:10 in absolute ethanol made a working stock solution.

FTr720

Lyophilised FTY720 (Novartis Biopharma) was dissolved into sterile milliQ water and frozen in $10 \mathrm{mg} / \mathrm{mL}$ aliquots at $-80^{\circ} \mathrm{C}$. FTY720 was further diluted for use to $50 \mu \mathrm{g} / \mathrm{mL}$. FTY720 was administered by daily gavage at a dose of $10 \mathrm{mg} /$ mouse unless.

\subsubsection{Flow cytometry reagents}

\section{Sodium azide}

NaN3 was purchased in powder form from Sigma-Aldrich New Zealand, Ltd (Auckland, NZ, S8032) and dissolved in dPBS to give a stock concentration of 5\%. The solution was stored at room temperature.

Fluorescent activated cytometry (flow) buffer

$0.01 \% \mathrm{NaN} 3$ and $2 \%$ FBS were made up in $\mathrm{dPBS}$ and stored at $4^{\circ} \mathrm{C}$.

Fluorescent activated cell-sorting (FACS) buffer

$0.002 \%$ DNase I and 2\% FBS were made up in dPBS, filter sterilised and stored at $4^{\circ} \mathrm{C}$.

4', 6-diamidino-2-phenylindole dihydrochloride (DAPI)

DAPI was purchased from Invitrogen (Auckland, NZ D1306) and resuspended in $\mathrm{dH}_{2} \mathrm{O}$ to a stock concentration of $5 \mathrm{mg} / \mathrm{mL}$ and stored in aliquots at $-80^{\circ} \mathrm{C}$. Stock was diluted in flow buffer to a concentration of $200 \mathrm{mg} / \mathrm{mL}$ and stored protected from light at $4 \varnothing \mathrm{C}$. 
LIVE/DEAD ${ }^{\circledR}$ Fixable blue dead cell Stain kit

LIVE/DEAD ${ }^{\circledR}$ Fixable blue was purchased from Invitrogen (Auckland, NZ L-23105) and stored protected from light at $-20^{\circ} \mathrm{C}$. Each vial was resuspended with room temperature $50 \mu \mathrm{L}$ anhydrous DMSO (component B of the kits) to forma working solution.

Anti-Mouse Ig, $\kappa /$ Negative control compensation particle

Mouse antibody binding compensation beads were purchased from Becton Dickinson Pty Ltd (Auckland, NZ, 552843) and stored at $4^{\circ} \mathrm{C}$.

Anti-Rat/Hamster Ig, $\kappa /$ Negative control compensation particle

Mouse antibody binding compensation beads were purchased from Becton Dickinson Pty Ltd (Auckland, NZ, 552845) and stored at $4^{\circ} \mathrm{C}$.

Carboxyfluorescein succinimidyl ester (CFSE)

CFSE was purchased from Molecular Probes (Eugene, OR, USA) and resuspended in was stored at $-20^{\circ} \mathrm{C}$, as a $10 \mathrm{mM}$ solution in DMSO Sigma-Aldrich New Zealand Ltd (Auckland, NZ).

\section{FoxP3 Transcription Factor Staining Set}

The FoxP3 transcription factor staining set (Affymetirx, eBioscience San Diego, CA, USA) was stored at $4{ }^{\circ} \mathrm{C}$. $4 \mathrm{x}$ Fixation/Permeabilization Concentrate was diluted 1:4 in Fixation/Permeabilization Diluent immediately prior to use. 10x Permeabilization Buffer was diluted 10 fold in $\mathrm{ddH}_{2} \mathrm{O}$ immediately prior to use, or diluted and stored at 4 ${ }^{\circ} \mathrm{C}$.

\section{IL-4 extracellular capture reagent}

Mouse IL-4 secretion assay detection kit was purchased from Miltenyi Biotec (Bergisch, Germany, 130-090-479) and stored at $4^{\circ} \mathrm{C}$. 
2.1.2.8 Fluorescently conjugated antibodies

\begin{tabular}{|c|c|c|c|}
\hline Specificity & Fluorophore & Glone & Company \\
\hline \multirow[t]{4}{*}{ B220 } & Biotin & RA3-6B2 & In house \\
\hline & Horizon V450 & RA3-6B2 & BD Biosciences \\
\hline & PE-Texas Red & RA3-6B2 & BD Biosciences \\
\hline & PE-CF594 & RA3-6B2 & BD Biosciences \\
\hline CD2 & Biotin & RM2-5 & Biolegend \\
\hline \multirow[t]{5}{*}{ CD3 } & Biotin & $2 \mathrm{C} 11$ & eBiosciences \\
\hline & BV786 & 145-2C11 & BD Horizon \\
\hline & $\mathrm{PE}$ & 145-2C11 & eBiosciences \\
\hline & PerCP-Cy5.5 & 145-2C11 & BD Pharmingen \\
\hline & PE-Cy7 & $2 \mathrm{C} 11$ & eBiosciences \\
\hline \multirow[t]{6}{*}{ CD4 } & Biotin & GK1.5 & In house \\
\hline & FITG & RM4-4 & BD Pharmingen \\
\hline & BV605 & RM4-5 & BD Horizon \\
\hline & APG & RM4-5 & BD Pharmingen \\
\hline & APC-H7 & GK1.5 & BD Pharmingen \\
\hline & Qdot605 & RM4-5 & Invitrogen \\
\hline \multirow[t]{4}{*}{ CD8 } & Biotin & 2.43 & In house \\
\hline & AF700 & $53-6.7$ & Biolegend \\
\hline & $\mathrm{PE}$ & 53-6.7 & BD Pharmingen \\
\hline & PE-Cy7 & $53-6.7$ & BD Pharmingen \\
\hline GD11a & PE-Gy7 & 2D7 & BD Pharmingen \\
\hline \multirow[t]{3}{*}{ CD11b } & FITC & $\mathrm{M} 1 / 70$ & BD Pharmingen \\
\hline & $\mathrm{PE}$ & $\mathrm{M} 1 / 70$ & BD Pharmingen \\
\hline & PerCP-Cy5.5 & $\mathrm{M} 1 / 70.15$ & BD Pharmingen \\
\hline \multirow[t]{2}{*}{ CD11c } & $\mathrm{PE}$ & HL3 & BD Pharmingen \\
\hline & PE-Gy7 & HL3 & BD Pharmingen \\
\hline CD16/CD32 & APC & $2.4 \mathrm{G} 2$ & BD Pharmingen \\
\hline CD19 & APC-H7 & ID3 & BD Pharmingen \\
\hline \multirow[t]{2}{*}{ CD25 } & FITC & 7D4 & BD Pharmingen \\
\hline & APG & PC61 & BD Pharmingen \\
\hline CD34 & BV421 & MEC14.7 & Biolegend \\
\hline
\end{tabular}




\begin{tabular}{|c|c|c|c|}
\hline CD40 & $\mathrm{PE}$ & $3 / 23$ & BD Pharmingen \\
\hline \multirow[t]{3}{*}{ CD44 } & PE & IM7 & BD Pharmingen \\
\hline & APG & IM7 & BD Pharmingen \\
\hline & AF700 & IM7 & BD Pharmingen \\
\hline \multirow[t]{2}{*}{ CD45 } & Pacific Blue & 30-F11 & Biolegend \\
\hline & APC-Cy 7 & 30-F11 & BD Pharmingen \\
\hline CD45.2 & APC & 104 & Biolegend \\
\hline CD49b & PE-Cy7 & DX5 & eBiosciences \\
\hline \multirow[t]{3}{*}{ CD62L } & Biotin & MEL-14 & BD Pharmingen \\
\hline & FITC & MEL-14 & BD Pharmingen \\
\hline & PE-Gy7 & MEL-14 & eBiosciences \\
\hline \multirow[t]{2}{*}{ CD69 } & $\mathrm{PE}$ & $\mathrm{H} 1.2 \mathrm{~F} 3$ & BD Pharmingen \\
\hline & PE-Cy7 & H1.2F3 & BD Pharmingen \\
\hline CD80 & APG & 16-10A1 & Biolegend \\
\hline CD86 & $\mathrm{PE}$ & GL1 & BD Pharmingen \\
\hline \multirow[t]{2}{*}{ CD90.2 } & BV605 & $53-2.1$ & Biolegend \\
\hline & APG & $53-2.1$ & BD Pharmingen \\
\hline CD95 & PE-CF594 & Jo2 & BD Horizon \\
\hline CD103 & APC & $2 \mathrm{E} 7$ & eBiosciences \\
\hline CD117 & APC & 2B8 & BD Pharmingen \\
\hline CD121b & $\mathrm{PE}$ & $4 \mathrm{E} 2$ & BD Pharmingen \\
\hline CD122 & $\mathrm{PE}$ & $\mathrm{TMbl}$ & BD Pharmingen \\
\hline \multirow[t]{2}{*}{ CD127 } & $\mathrm{PE}$ & SB199 & BD Pharmingen \\
\hline & PE-Cy7 & A7R34 & Biolegend \\
\hline CD152 & $\mathrm{PE}$ & UC10-4F10-11 & BD Pharmingen \\
\hline CD154 & $\mathrm{PE}$ & MR1 & BD Pharmingen \\
\hline CD200R3 & APG & Ba13 & Biolegend \\
\hline CD278 & PE & C398.4A & Biolegend \\
\hline CD279 & PerCP-ef7 10 & RMPI-30 & eBiosciences \\
\hline CXCR5 & APC & $2 \mathrm{G} 8$ & BD Pharmingen \\
\hline \multirow[t]{2}{*}{ FсعRI } & Biotin & MAR-1 & Biolegend \\
\hline & $\mathrm{PE}$ & MAR-1 & eBiosciences \\
\hline
\end{tabular}




\begin{tabular}{|c|c|c|c|}
\hline GATA-3 & AF647 & L50-823 & BD Pharmingen \\
\hline \multirow[t]{2}{*}{ huCD2 } & $\overline{\mathrm{APG}}$ & RPA2.10 & BD Pharmingen \\
\hline & $\mathrm{PE}$ & S5.2 & BD Pharmingen \\
\hline \multirow[t]{2}{*}{ IA/IE(MHG II) } & $\mathrm{PE}$ & M5/114.15.2 & BD Pharmingen \\
\hline & PE-Gy7 & M5/114.15.2 & BD Pharmingen \\
\hline $\operatorname{IgE}$ & Biotin & R35-118 & BD Pharmingen \\
\hline IL-4 & AF647 & $11 \mathrm{~B} 11$ & BD Pharmingen \\
\hline IL-13 & AF647 & eBiol3A & eBiosciences \\
\hline KLRG1 & $\mathrm{APG}$ & $2 \mathrm{~F} 1$ & BD Horizon \\
\hline Ly6A/E & $\mathrm{PE}$ & D7 & Biolegend \\
\hline \multirow[t]{2}{*}{ Ly6C/Ly6G (GR1) } & Biotin & RB6-8C5 & In house \\
\hline & $\mathrm{APG}$ & RB6-8C5 & BD Pharmingen \\
\hline \multirow[t]{2}{*}{ NK1.1 } & $\mathrm{PE}$ & PK136 & BD Pharmingen \\
\hline & PerCP-Gy5.5 & PK136 & BD Pharmingen \\
\hline Siglec F & $\mathrm{PE}$ & E50-2440 & BD Pharmingen \\
\hline T1 ST2 & FITG & DJ8 & MD Bioproducts \\
\hline $\mathrm{V} \alpha 2$ & $\mathrm{APG}$ & B20.1 & Invitrogen \\
\hline $\mathrm{V} \beta 5.1 / 5.2$ & Biotin & MR9-4 & BD Pharmingen \\
\hline
\end{tabular}

\begin{tabular}{|l|l|}
\hline Fluorophore bound Streptavidin & Company \\
\hline SA-APC & BD Pharmingen \\
\hline SA-PE-Texas Red & BD Pharmingen \\
\hline SA-PE-Cy7 & BD Pharmingen \\
\hline SA-QDot565 & Invitrogen \\
\hline
\end{tabular}

Table 2-1 - List of fluorescently labelled antibodies used in this thesis for flow cytometry.

Listed are the antibody specificities, fluorophore, clone and supplier.

\subsubsection{Polymerase chain reaction reagents}

4C13R primers

Gene target: DS-Red

5'-GGTCGAAGGTGTAGGTGAAG-3'

5'-GGTTGGAGTGCACGTAGTAG-3'

Produced an approximately 450 base pair product. Primers bought from Sigma-Aldrich

New Zealand Ltd (Auckland, NZ). 
Diethylpyrocarbonate (DEPC) treated water

UltraPure DEPG-Treated water from Invitrogen (Auckland, NZ, 10813-012) was stored at room temperature.

DNA polymerase kit

i-Taq DNA polymerase kit from iNtRON Biotechnology (Korea, 25022) was stored at $20^{\circ} \mathrm{G}$.

Agarose

Agarose from BDH Laboratory Supplies (Poole, UK) was stored in powder form at room temperature.

Gel loading dye

10x concentration BlueJuice gel loading buffer from Invitrogen (Auckland, NZ, 10816015) was stored at $4^{\circ} \mathrm{C}$.

SYBR safe DNA gel stain

SYBR safe DNA gel stain from Invitrogen (Auckland, NZ, S33102) was stored at room temperature.

DNA ladder

TrackIt $1 \mathrm{~Kb}$ plus DNA ladder from Invitrogen (Auckland, NZ, 10488-085) was stored at $4^{\circ} \mathrm{C}$.

\subsubsection{Mice}

\subsubsection{Maintenance and ethics approvals}

Mice were bred and maintained in specific pathogen free conditions within the Biomedical Research Unit of the Malaghan Institute of Medical Research. For most experiments six week or older mice were age and sex matched as much as possible. For age experiments mice younger than 6 weeks were culled for tissues without manipulation. Experimental procedures were approved by the Victoria University 
Animal Ethics Committee and carried out in accordance with the guidelines of Victoria University of Wellington, New Zealand. This work was performed under the following protocols:

2009R7M - Immunopathogenesis of food allergy

2009R14M - The role of cytokines and immune cells in protective immunity to parasites and inflammatory diseases

$2011 \mathrm{R} 10 \mathrm{M}$ - Novel vaccine approaches for protecting against parasitic helminths

2011R22M - Basic biology of the Th2 response in the context of allergic disease

2014R15M - Immunomodulation and Immune protection by helminth parasites and their products

2014R17M - Activation and role of novel Th2 subtypes in allergic disease

\subsubsection{Mouse strains}

Balb/c mouse strain

Obtained from Jackson Laboratory (Bar Harbour, Maine, USA) and maintained as an inbred strain.

\section{C57Bl/6 mouse strain}

Obtained from Jackson Laboratory (Bar Harbour, Maine, USA) and maintained as an inbred strain.

G4/G4 (GFP/GFP) mouse strain

Obtained from W. E. Paul at the Laboratory of Immunology, National Institute of Allergy and Infectious Diseases, National Institute of Health, Bethesda MD 20892-1892, $\mathrm{USA}^{227}$. Mice were originally on a $\mathrm{C} 57 \mathrm{Bl} / 6$ background but were crossed to a Balb/c background. Both strains were maintained as inbred strains.

G4/IL-4 (GFP/IL-4) mouse strain

This strain is also available on both $\mathrm{C} 57 \mathrm{Bl} / 6$ and Balb/c backgrounds. These mice were generated as the F1 offspring from crosses between the GFP/GFP mice and the appropriate WT strain, either Balb/c or $\mathrm{C} 57 \mathrm{Bl} / 6$. 
KN2/KN2 (huCD2/huCD2) mouse strain

Obtained from R. M. Locksley from the Howard Hughes Medical Institute, University of California, San Francisco, CA 94143-0795, USA ${ }^{289}$. Mice are on a Balb/c background and maintained as an inbred strain.

KN2/IL-4 (huCD2/IL-4) mouse strain

This strain was generated as F1 offspring between the $\mathrm{KN} 2 / \mathrm{KN} 2$ mice and Balb/c mice.

KN2/G4 (huCD2/GFP) mouse strain

This strain was generated as F1 offspring between the KN2/KN2 mice and G4/G4 mice.

\section{C13R mouse strain}

Obtained from W. E. Paul at the Laboratory of Immunology, National Institute of Allergy and Infectious Diseases, National Institute of Health, Bethesda MD 20892-1892, USA. Mice were generated as follows; BAC-clone (RP23.97H11) containing the Th2 locus control region (lcr), Il13, Il4, and Kif3 genes was obtained from the Children's Hospital of Oakland Research Institute. A galk cassette containing 5' and 3' homology arms from the Il4 gene inserted via homologous recombination targeted to the starting ATG codon of the Il4 gene. The resultant "Il4 galk" was then targeted with an AmCyan construct, which replaced galk. Using the same principle, the starting ATG codon of the $I l 13$ gene of the "Il4 AmCyan" BAC was targeted and replaced with a galk cassette. A DsRed-DR construct replaced the galk sequence. The BAC was linearized by digestion with Ascl and the fragment containing Th2 LCR, Il13-DsRed-DR, Il4AmCyan, and KIF3 was microinjected into C57Bl/6 oocytes. These oocytes were subsequently transferred into pseudo-pregnant foster mothers of $\mathrm{C} 57 \mathrm{Bl} / 6$ background. Offspring carrying the $4 \mathrm{C} 13 \mathrm{R}$ reporter construct were selected for by polymerase chain reaction $(\mathrm{PCR})^{265}$. The strain was maintained by crossing to $\mathrm{C} 57 \mathrm{Bl} / 6$ mice, offspring were tested by PCR and non-transgenic offspring kept for controls.

\section{C13RxG4/IL-4 (4C13RxGFP/IL-4) mouse strain}

This strain was generated as F1 offspring between the 4C13R mice and G4/G4 mice. Offspring were tested by PCR for presence of the 4C13R construct. 
4C13RxG4/G4 (4C13RxGFP/GFP) mouse strain

This strain was generated as F1 offspring between the 4C13RxG4/IL-4 mice and G4/G4 mice. Offspring were tested by PCR for presence of the 4G13R construct and by flow cytometry, assessing basophil GFP fluorescence to determine hetero- or homozygosity for the IL-4-GFP allele.

4C13RxKN2/G4 (4C13Rx huCD2/GFP) mouse strain

This strain was generated as F1 offspring between the 4G13RxG4/IL-4 mice and KN2/KN2 mice. Offspring were tested by PGR for presence of the 4G13R construct and by flow cytometry, assessing basophil GFP fluorescence to assess the presence of the IL-4-GFP allele.

\section{C13RxOTII mouse strain}

This strain was generated as F1 offspring between the 4C13R mice and OTII mice (Provided by S. Hook, School of Pharmacy, Otago University, Dunedin, New Zealand with permission from F. Carbone, Melbourne, Victoria, AUS) ${ }^{291}$. Offspring were tested by PCR for presence of the 4C13R construct.

Rag1 ${ }^{-/-}$mouse strain

Obtained from Jackson Laboratory (Bar Harbour, Maine, USA ${ }^{292}$. These mice were on a $\mathrm{C} 57 \mathrm{Bl} / 6$ background and maintained as an inbred strain.

\section{TSLPR $^{-/-}$x G4/G4 mouse strain}

TSLPR-/-xG4/G4 mice were obtained from Prof. William Paul (NIAID, NIH, Bethesda, USA), with permission from Prof. Warren Leonard (National heart, lung, and blood institute, NIH, Bethesda, USA)293. TSLPR-deficient mice were crossed with G4/G4 mice and the offspring crossed to generate TSLPR deficient mice that were homozygous for the GFP IL-4 reporter. These mice were on a C57Bl/6 background and maintained as an inbred strain.

eGFP mouse strain

Transgenic mice with the sequence for enhanced GFP under control for the beta actin promoter for widespread eGFP expression were obtained from Hercus Taieri Research 
Unit, (Dunedin, NZ)294. These mice were on a C57Bl/6 background and maintained as an inbred strain.

DS-Red mouse strain

Transgenic mice with the sequence for DS-Red under the control of the chicken beta actin promoter coupled with the cytomegalovirus (CMV) immediate early enhancer for widespread DS-Red expression were obtained from Jackson Laboratory (Bar Harbour, Maine, USA) 295 these mice were on a C57Bl/6 background and maintained as an inbred strain.

\subsubsection{Nippostrongylus brasiliensis}

Nippostrongylus brasiliensis from L. A. Dent (Adelaide, South Australia, AUS) in 1995 has maintained by passage through Lewis rats as described ${ }^{124}$. 


\subsection{Methods}

\subsubsection{Trypan blue exclusion assay}

Cell suspensions were mixed 1:1 with Trypan blue dye. 10ul of the mixture was transferred to a haemocytometer and cell numbers counted on a compound microscope.

\subsubsection{Hybridoma culture}

Hybridoma cells cultured in cIMDM with optimised concentrations of FBS (either 10\% or $20 \%$ ) were seeded at $2 \times 10^{6}$ cells $/ \mathrm{mL}$ into the cell compartments of the CELLine flasks. The nutrient compartments were filled with 5\% cIMDM. After one week supernatant from the cell compartment was collected, filtered through Serum filters and antibodies isolated from the supernatant by fast pressure liquid chromatography using Hitrap protein $G$ affinity columns. Antibodies were dialysed in dPBS using 1000 molecular weight cut-off (MWCO) cassettes. Concentration of antibody was measured using Nanodrop absorption readings at $280 \mathrm{~nm}$. Antibody was concentrated using 10000 MWCO Vivaspin tubes.

\subsubsection{Genotyping}

\subsubsection{4C13R strains}

PCR genotyping was performed on DNA extracted from tail tips. Tails were hydrolysed in tail lysis buffer at $95^{\circ} \mathrm{C}$ for 2 hours to extract DNA, hydrolysis was quenched with neutralisation buffer. PCR was performed with the appropriate primers using the i-Taq DNA Polymerase kit. PGR products were run on 1.8\% Agarose gel, loaded with 10X BlueJuice Gel Loading Buffer and visualised with SYBR Safe DNA Gel Stain. TrackIt 1 Kb Plus DNA Ladder was used to determine PGR product size.

\subsubsection{G4 (GFP) strains}

Blood was collected and processed as in 2.2.7.8. After red blood cell lysis, cells were stained with antibodies against FceRI, CD49b and CD45 to identify basophils and analysed via flow cytometry. Basophils were identified by the expression of CD45 ${ }^{\text {mid }} \mathrm{CD} 49 \mathrm{~b}^{+} \mathrm{FcERI}^{+}$and GFP median fluorescent intensity of basophils was 
compared to control samples from G4/IL-4 and G4/G4 mice to allow classification of mice as GFP/IL-4 and GFP/GFP.

\subsubsection{Preparation of infectious larvae}

Infective L3 were harvested from faecal cultures, larvae were washed in sterile dPBS, allowed to settle and the dPBS taken off. This was repeated three times then the larvae were counted and the sample was diluted to 11000 larvae/mL $(550 / 50 \mu \mathrm{L})^{124}$.

\subsubsection{Mouse manipulations}

\subsubsection{Serum Collection}

Blood was collected by lateral tail vein into a $1.7 \mathrm{~mL}$ Microtubes and left to clot overnight at $4^{\circ} \mathrm{C}$. Blood was spun for 3 minutes at 4 degrees at $1523 \mathrm{xg}$ and serum was removed and frozen at $-20^{\circ} \mathrm{C}$ until analysed.

\subsubsection{Nippostrongylus brasiliensis infection}

All infections were via the intravenous route unless otherwise stated. Washed larvae from 2.2.4 were injected subcutaneously in the scruff of the neck or intravenously into the tail vein. For intravenous injection mice were heated under a heat lamp for a short time (2-4 minutes) until tail veins were readily visible. For secondary infections primary infected mice were left for 30 days to clear the adult worms from the intestine. On day 30 post-primary the mice were re-infected either subcutaneously or intravenously. ${ }^{124}$

\subsubsection{Primary Th2 immune response assay (pTh2 assay)}

IL-4 reporter mice were anesthetised with $200 \mu \mathrm{L}$ of working concentration anaesthetic. Mice received $30 \mu \mathrm{L}$ house dust mite $(100 \mu \mathrm{g})$ intradermally into the pinna of each ear. At harvest the auricular draining lymph nodes were excised and collected into plates with flow buffer on ice. ${ }^{296}$ 


\subsubsection{MC903}

Mice were anesthetised with $200 \mu \mathrm{L}$ of working concentration anaesthetic. $10 \mu \mathrm{L}$ of working concentration MC903 was applied to each side of the mice ears $(20 \mu \mathrm{L} /$ ear $) .{ }^{297}$

\subsubsection{Tape striping}

Mice were anesthetised with $200 \mu \mathrm{L}$ of working concentration anaesthetic. Masking tape from Office Max (Wellington, New Zealand) was applied to the dorsal side of each ear and pulled off in a quick motion; this was repeated 20 times/ear with a new piece of tape for each strip. ${ }^{298}$

\subsubsection{Antibody administration}

\section{CD4 depletion}

Stock GK 1.5 antibody was diluted with sterile dPBS to $2.5 \mathrm{mg} / \mathrm{mL} 0.5 \mathrm{mg}$ antibody was administered intraperitoneally ${ }^{125}$. Mice received GK1.5 either 24 hours before primary infection or 24 hours before secondary infection (Day -1) or 7 days and 3 days before secondary infection (Day -7).

\section{CD90 depletion}

Stock $30 \mathrm{H} 12$ antibody was diluted with sterile dPBS to $2.5 \mathrm{mg} / \mathrm{mL} 0.5 \mathrm{mg}$ antibody was administered intraperitoneally ${ }^{299}$. For ILC2 depletion in Rag1/- mice, mice were treated daily for 5 days prior to primary infection and once again 24 hours post infection.

\section{IL-13R blocking}

Stock was diluted to $5 \mathrm{mg} / \mathrm{mL}$ with sterile dPBS was administered intraperitoneally.

\subsubsection{Cytokine administration}

Intranasal IL-33

Mice were anesthetised with $200 \mu \mathrm{L}$ of working concentration anaesthetic and were laid on their back. $50 \mu \mathrm{L}$ of IL-33 $(0.5 \mu \mathrm{g})$ was carefully pipetted into the nostrils of each mouse $^{300}$. For the cytokine protection models mice were treated 2 days prior to infection. 
IL-2 complex $(I L-2 c)$

Stock IL-2c was thawed and diluted 1:16.67 (30:500 $\mu \mathrm{L})$ in sterile dPBS. Mice received $100 \mu \mathrm{L}$ intraperitoneally ${ }^{301}$. For the cytokine protection models mice were treated 2 days prior to infection. For rescuing protection by ILC2 stimulation in CD4 ${ }^{+} \mathrm{T}$ cell depleted mice, mice were treated three times every second day beginning 6 days prior to secondary infection.

\subsubsection{Tissue processing for larval counts}

Mice were sacrificed by cervical dislocation and lungs excised. Lungs are collected in plates with no liquid. Lungs were then mechanically dissociated, placed in cheesecloth and suspended in a $50 \mathrm{~mL}$ Falcon tube of dPBS and placed in a $37^{\circ} \mathrm{C}$ water bath overnight. Viable larvae migrate out of the tissue and collect at the base of the tube, these can be enumerated using a stereo microscope ${ }^{124}$.

\subsubsection{Tissue processing for single cell suspension}

\subsubsection{Spleen for flow cytometry and Th polarising cultures}

Mice were sacrificed by $\mathrm{CO}_{2}$ asphyxiation and spleen excised and placed in plates with IMDM on ice. Under sterile conditions spleens were dissociated by crushing with the plunger from $2 \mathrm{~mL}$ syringe through a $70 \mu \mathrm{m}$ cell strainer and washed through with IMDM. Cells were pelleted at $419 \times \mathrm{g}$ for 4 minutes. Pellet was resuspended in $4 \mathrm{~mL}$ Red Blood Cell Lysis buffer for 4 minutes. Lysis was quenched with $10 \mathrm{~mL}$ of cold IMDM and cells pelleted the same as before. Cells were resuspended in cIMDM for counting.

\subsubsection{Spleen for antigen presenting cells}

Mice were sacrificed by $\mathrm{CO}_{2}$ asphyxiation and spleen excised and placed in plates with IMDM on ice. Under sterile conditions spleens were places in $3 \mathrm{~mL}$ spleen digestion buffer (2.1.2.2) and mechanically dissociated and incubated at $37^{\circ} \mathrm{C}$ for 30 minutes. Cells were then passed through a $70 \mu \mathrm{m}$ cell strainer and washed through with IMDM. 
Cells were pelleted at $419 \mathrm{x}$ g for 4 minutes. Pellet was resuspended in $4 \mathrm{~mL}$ Red Blood Cell Lysis buffer for 4 minutes. Lysis was quenched with $10 \mathrm{~mL}$ of cold IMDM and cells pelleted the same as before. Cells were resuspended in cIMDM for counting.

\subsubsection{Lungs}

Mice were sacrificed by cervical dislocation and lungs were excised and placed in plates with IMDM on ice. Lungs were finely cut and incubated in $5 \mathrm{~mL}$ of lung diction buffer at $37^{\circ} \mathrm{C}$ for 60 minutes. Digested tissue was passed repeatedly through an 18-gauge needle then passed through a $70 \mu \mathrm{m}$ cell strainer and washed through with IMDM. Cells were pelleted at $419 \mathrm{x}$ g for 4 minutes. Pellet was resuspended in $4 \mathrm{~mL}$ Red Blood Cell Lysis buffer for 4 minutes. Lysis was quenched with 10mL of cold IMDM and cells pelleted the same as before. Cells were resuspended in flow buffer for counting.

\subsubsection{Thymus}

Mice were sacrificed by $\mathrm{CO}_{2}$ asphyxiation and thymi excised and placed in plates with IMDM on ice. Under sterile conditions thymi were dissociated by crushing with the plunger from $2 \mathrm{~mL}$ syringe through a $70 \mu \mathrm{m}$ cell strainer and washed through with IMDM. Cells were pelleted at $419 \mathrm{x} g$ for 4 minutes. Cells were resuspended in flow buffer for counting.

\subsubsection{Lymph Nodes}

Mice were sacrificed by $\mathrm{CO}_{2}$ asphyxiation (pTh2 auricular draining LN) or cervical dissociation (mediastinal LN) and LNs excised and placed in plates with IMDM on ice. Under sterile conditions LNs were dissociated by crushing with the plunger from $2 \mathrm{~mL}$ syringe through a $70 \mu \mathrm{m}$ cell strainer and washed through with IMDM. Cells were pelleted at $419 \mathrm{x}$ g for 4 minutes. Cells were resuspended in flow buffer for counting.

\subsubsection{DynaBead CD4 isolation}

$5 \times 10^{7}$ cells from single cell suspensions from the above processing were spun down at $419 \mathrm{x} \mathrm{g}$ for 4 minutes and resuspended and incubated in isolation buffer with $25 \mu \mathrm{L}$ of $\alpha \mathrm{CD} 4$ antibody (from the Dynabeads CD4 positive isolation kit, 2.1.2.2) for 10 minutes 
on ice. These cells were washed with isolation buffer, pelleted and resuspended in isolation buffer with $75 \mu \mathrm{L}$ Dynabead mix was added and incubated rolling and tilting for 15 minutes at $4^{\circ} \mathrm{C}$. Bead bound cells were gathered to one side of the tube using magnets and the remaining suspended cells were aspirated away. Bead bound cells were resuspended in isolation buffer and then magnetic separation was repeated. Bead bound cells were resuspended in $1 \mathrm{~mL}$ release buffer and incubated rolling at tilting for 10 minutes at room temperature. Tubes were placed on the magnet again and suspended $\mathrm{CD}^{+} \mathrm{T}$ cells were removed by pipette. These cells were pelleted, resuspended in cIMDM and then prepared for culture.

\subsubsection{Macs bead CD11c isolation}

$10^{7}$ cells from spleens digested as in 2.2.7.2 were pelleted down at $419 \mathrm{x} \mathrm{g}$ for 4 minutes and resuspended and incubated in $100 \mu \mathrm{L}$ of $\alpha \mathrm{CD} 11 \mathrm{c}$ for 15 minutes on ice. Cells were washed in isolation buffer and spun down and resuspended in $1 \mathrm{~mL}$ isolation buffer. Samples were run on the AutoMACs separator using a POSSEL programme for the positive selection of $\mathrm{CD} 1 \mathrm{c}^{+}$cells. Collected cells were pelleted and resuspended in cIMDM for counting.

\subsubsection{Blood}

Blood was collected via tail vein bleeding, and collected in Alsever's solution. Cells were pelleted at $630 \mathrm{x}$ g and supernatant aspirated. Pellets were resuspended in $\mathrm{H}_{2} \mathrm{O}$ for 30 seconds then adding equal volume of $1.8 \% \mathrm{w} / \mathrm{v} \mathrm{NaCl}$ solution quenched the lysis. Cells were re-pelleted and resuspended in flow buffer for counting.

\subsubsection{Skin}

Ears were excised and the ventral and dorsal halves of the pinna split using tweezers and mechanically dissociated then incubated in skin digestion buffer for 1 hour shaking at $150 \mathrm{rpm}$ at $37^{\circ} \mathrm{C}$. Digested tissue was worked through $80 \mu \mathrm{m}$ stainless steel mesh with the plunger from a 1mL syringe. Cells were washed through with flow buffer. Cells were washed twice with flow buffer and resuspended in flow buffer for counting. 


\subsubsection{Th cell polarisation culture}

24 well plates were coated overnight at $4^{\circ} \mathrm{C}$ with $300 \mu \mathrm{L} /$ well of $1 \mu \mathrm{g} / \mathrm{mL} \alpha \mathrm{CD} 3$ (2C11) diluted in dPBS. On day 0 plates were washed twice with IMDM and $10^{6}$ splenocytes or $2 \times 10^{5}$ Dynabead enriched CD4 $\mathrm{T}$ cells in a $1 \mathrm{~mL}$ total volume of the appropriate stimulatory media were plated. Half the media was replaced with appropriate feed media on days 3 and 4 .

On day 5 cells were harvested spun down at 419 x g for 4 minutes. Cells were resuspended in cIMDM with $100 \mathrm{U} / \mathrm{mL}$ IL-2 and plated on uncoated 24 well plates at $10^{6}$ cells/well to rest. Half the media was replaced daily from day 6-10 with cIMDM with 200U/mL IL-2. On day 11 cells were harvested again and plated in media containing $100 \mathrm{U} / \mathrm{mL}$ of IL-2 and 1:50 $\alpha \mathrm{CD} 28$ (37.51) on plates that had been coated overnight with $10 \mu \mathrm{g} / \mathrm{mL} \alpha \mathrm{CD} 3$ at $4^{\circ} \mathrm{C}^{30}$.

\subsubsection{Th0 media}

Stimulation Media

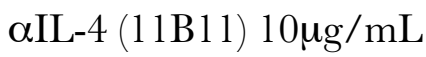

$\alpha \mathrm{IFN}-\gamma(\mathrm{AN}-18) 10 \mu \mathrm{g} / \mathrm{mL}$

$\alpha \mathrm{CD} 28$ (37.51) 1:50

Feed media

IL-2 200U/mL

IL-4 2000U/mL

\subsubsection{Th2 media}

Stimulation Media

IL-2 100U/mL

IL-4 1000U/mL

$\alpha$ CD28 (37.51) 1:50

Feed media

IL-2 200U/mL

IL-4 2000U/mL 


\subsubsection{Carboxyfluorescein succinimidyl ester (CFSE) staining}

Stock CFSE was diluted in $\mathrm{dPBS}$ to $0.5 \mu \mathrm{M}$ then added to single cell suspension in $\mathrm{dPBS}$ at a ratio of 1:4 cell suspension : diluted CFSE solution. Cells were incubated for 5 minutes protected from light at room temperature. Adding an equivalent volume of FBS quenched staining. Cells were pelleted at $419 \mathrm{x} g$ for 4 minutes and washed twice in cIMDM. ${ }^{302}$ Cells were then be used for culture.

\subsubsection{Antigen processing cell - OTIl cell culture}

APGs from digested spleens (2.2.7.2) were incubated for 30 minutes with 10nM ISQ peptide in cIMDM at $37^{\circ} \mathrm{C} .{ }^{291}$ APGs were washed with IMDM twice, and then resuspended in cIMDM. APGs were mixed in a ratio of 1:1 with Dynabead isolated CD4 T cells (2.2.7.6) from OTII mice and cultured in the presence of $100 \mathrm{U} / \mathrm{mL}$ IL-2 and $1000 \mathrm{U} / \mathrm{mL}$ IL-4. Half the media was replaced on day 3 with feed media containing 200U/mL IL-2 and 2000U/mL IL-4.

For restimulation on day 3 spleens were digested (2.2.7.2) and $\mathrm{CD} 11 \mathrm{c}^{+}$cells isolated via AutoMAGs (2.2.7.7). CD11c cells were incubated for 30 minutes with 10nM ISQ peptide in cIMDM at $37^{\circ} \mathrm{C}$. These cells were then washed in IMDM and resuspended in cIMDM containing 200U/mL IL-2 and 2000U/mL IL-4. Half of restimulated wells media was replaced with the $\mathrm{CD} 11 \mathrm{c}^{+}$cell suspension.

\subsubsection{Enzyme-linked immunosorbent assay (ELISA)}

Nunc Maxisorb 96 well plates were coated with $100 \mu \mathrm{L}$ of the appropriate coating antibody and incubated overnight at $4^{\circ} \mathrm{C}$. Plates were washed by submersion into a $4 \mathrm{~L}$ bucket containing dPBS and 0.05\% Tween-20 Sigma-Aldrich New Zealand Ltd (Auckland, NZ, P2287), excess liquid was removed from the wells by smacking the inverted plate on a pile of paper towels. Plates were blocked with $10 \%$ FBS in dPBS solution for 2 hours at $37^{\circ} \mathrm{C}$. Samples were serially diluted in 10 fold dilution steps with $10 \%$ FBS in d solution. Standards were serially diluted across 11 wells in 2 fold steps in the same $10 \%$ FBS in dPBS solution. Block was washed from the plates and $100 \mu \mathrm{L}$ of samples and standards were incubated in the plates overnight at $4^{\circ} \mathrm{C}$. Samples and standards were washed out and the $100 \mu \mathrm{L}$ of the appropriate biotin bound detection antibody was added to the plates. Detection antibodies were washed out and $100 \mu \mathrm{L}$ of 
streptavidin horse radish peroxidase diluted in 10\% FBS in dPBS solution and incubated for a further hour at $37^{\circ} \mathrm{C}$. Plates were washed and developed with $100 \mu \mathrm{L}$ of a 1:1 mix of TMB A and B substrates, and development was stopped with 100 $\mu \mathrm{L}$ of $1 \mathrm{M}$ $\mathrm{H}_{2} \mathrm{SO}_{4}$. Absorbance was read at 450nm on the Versamax Microplate reader.

\subsubsection{IL-4 ELISA}

Capture antibody $-11 \mathrm{~B} 11(2 \mu \mathrm{g} / \mathrm{mL})$

Detection antibody - $\alpha$ IL-4-biotin $(2 \mu \mathrm{g} / \mathrm{mL})$

\subsubsection{IgE ELISA}

Capture antibody - 6HD5 $(5 \mathrm{mg} / \mathrm{mL})$

Detection antibody - R1E4-biotin $(3 \mu \mathrm{g} / \mathrm{mL})$

\subsubsection{Flow cytometry staining and analysis}

\subsubsection{Surface staining}

$1.5 \times 10^{6}$ cells (or $2 \times 10^{6}$ cells for ILC analysis) were transferred into $5 \mathrm{~mL}$ polystyrene round bottom tubes through $70 \mu \mathrm{m}$ gauze, and cells were pelleted at $419 \mathrm{x} \mathrm{g}$ for 4 minutes. Cells were resuspended in 50 $\mu \mathrm{L}$ of $\alpha \mathrm{CD} 16 / 32$ (2.4G2) and cells were incubated for 5 minutes on ice. $50 \mu \mathrm{L}$ of the fluorophore bound antibody cocktail was then added to the tubes that were incubated for another 30 minutes on ice. Staining was stopped by the addition of $4 \mathrm{~mL}$ of flow buffer and spun down. For secondary staining pellets were resuspended in $100 \mu \mathrm{L}$ of secondary antibodies diluted in flow buffer and incubated on ice for 15 minutes prior to washing. Samples were resuspended in $125 \mu \mathrm{L}$ flow buffer and prior to analysis $125 \mu \mathrm{L}$ of DAPI solution was added to each tube.

\subsubsection{Intracellular staining (ICS)}

$1.5 \times 10^{6}$ cells (or $2 \times 10^{6}$ cells for ILC analysis) were transferred into $5 \mathrm{~mL}$ polystyrene round bottom tubes through $70 \mu \mathrm{m}$ gauze, and cells were pelleted at $419 \mathrm{x} \mathrm{g}$ for 4 minutes. Cells were resuspended in $1 \mathrm{~mL}$ diluted LIVE/DEAD fixable blue in dPBS 
and incubated for 30 minutes at $4^{\circ} \mathrm{C}$. Cells were pelleted and stained as per 2.2.10.1. After surface staining and washing pellets were resuspended in $1 \mathrm{~mL}$ of diluted Fixation/Permeabilization buffer (from FoxP3 Transcription Factor Staining buffer set) and incubated for 30 minutes at $4^{\circ} \mathrm{C}$ protected from light. Cells were washed once with 1x-diluted Permeabilization solution, pelleted then resuspended and incubated in $100 \mu \mathrm{L} 1 \mathrm{x}$-diluted Permeabilization solution with intracellular staining antibody at room temperature overnight. Cells were washed twice with 1x-diluted Permeabilization solution then finally resuspended in $250 \mu \mathrm{L}$ of flow buffer.

\subsubsection{IL-4 extracellular capture assay}

$10^{6}$ ells from culture (2.2.8) were collected and washed in $20 \mathrm{~mL}$ of cold cIMDM then pelleted at $300 \mathrm{x} g$ for 10 minutes. Pallets were resuspended in a 1:10 mixture of the IL4 catch reagent from the Mouse IL-4 secretion assay detection kit (Miltenyi Biotec Bergisch, Germany) diltued in cIMDM and incubated on ice for 5 minutes. 20mL of pre-warmed IMDM was added to the cells and cells were cultured rolling and tilting for 45 minutes at $37^{\circ} \mathrm{C}$. Cells were repelletted and washed twice in cold flow cytometry buffer. Cells were then stained as per 2.2.10.1 with the IL-4 detection antibody from the Mouse IL-4 secretion assay detection kit added to the staining cocktail.

\subsubsection{Acquisition}

Flow Cytometry data was acquired using a BD LSRII special order research product (SORP) and a BD LSR Fortessa with a unique laser arrangement specifically modified for optimal detection of AmCyan (Blue Violet 445nm laser with 504/12 filter). Both machines were running BD FACSDiva software. Cytometer setup and tracking was performed daily to ensure machines were calibrated. See appendix for laser and detector configurations (Table 8-1).

\subsubsection{Compensation}

For accurate compensation single stain controls were stained at the same time as surface staining (2.2.10.1). The appropriate anti-mouse or anti-rat/hamster Ig, k/Negative control compensation beads were singly stained with antibodies conjugated to the same fluorophores as those used in the experimental staining cocktails. A mouse anti-human CD45-AmCyan antibody was used as a single stain control for the AmCyan-IL-4 fluorescent reporter. Splenocytes processed as in 2.2.7.1 from eGFP and DS-Red 
constitutively expressing mice were used as single stain controls of GFP-IL-4, and DSRed-IL-13 fluorescent reporters. Compensation was applied using the automatic compensation wizard in the FACSDiva software.

\subsubsection{Fluorescence activated cell sorting}

Cells were sorted by the staff of Hugh Green Cytometry Core on a FACSVantage cell sorter, using DIVA software (Becton-Dickinson, Mountain View, CA, USA).

\subsubsection{Analysis of flow cytometry data}

Data was analysed using FlowJo software (Tree Star, Ashland, Oregon, USA). Compensation was reviewed by observing each single stain control against every other collected channel and confirming there was no over or under compensation applied. Singlets were selected for by gating for cells on the diagonal in FSChight by FSCarea and SSChight by SSCarea plots. Dead cells were excluded by gating on DAPI- cells. For specific gating on each leukocyte population see appendix. For gating upon populations where there was no clear division between positive and negative cells fluorescence minus one (FMO) controls were used to determine where background staining in each channel was and where gates should be set.

\subsubsection{Statistical analysis}

Data were graphed and analysed in Graphpad Prism 5.0. Statistical analyses used are stated in figure legends and $\mathrm{p}<0.05$ was considered as significant. In comparisons between two similarly-sized groups, Student's t-test was used. Analyses examining a single parameter of three or more groups were compared with One-way ANOVA with Tukey's post-test. For experiments examining two or more groups, Two- way ANOVA with Bonferroni's post-test were used. Repeated measures tests were used in cases in which the same biological sample was compared. Unless otherwise stated, symbols represent individual samples and error bars represent mean \pm the standard error of the mean (S.E.M.). 


\section{Mechanisms expression of IL-4 by $\mathrm{CD}^{+} \mathrm{T}$ cells}

\subsection{Introduction}

IL-4 production by CD4 ${ }^{+} \mathrm{T}$ cells is critical for Type 2 immune responses and while they have been studied for over 25 years the mechanisms that regulate IL-4 expression remain obscure. This chapter will introduce and characterise a new reporter mouse strain and explores how Th2 cells regulate their expression of IL-4, including what signals affect the proportions of Th2 cells expressing IL-4 mono- and bi-allelically, and the importance of IL-4 to in vivo Th2 generation.

To study the allelic nature of IL-4 expression two strains, the human CD2 (huCD2)289 and the GFP227 IL-4 reporter mice strains have been crossed in this laboratory to allow the reporting of which IL-4 allele a Th2 cell is actively transcribing from. Utilising this system mono- and bi-allelically expressing Th2 cells can be identified, characterised and tracked over time. This chapter explores the nature of signals that drive production of IL4 from both as opposed to one allele and how stable these phenotypes are.

IL-4 reporter mice have provided significant insight into the regulation of Th2 cytokine production, however the available knock-in reporter systems have many caveats that can potentially affect the Th2 and Type 2 immune responses that they are reporting. Most knock-in reporter mice systems, such as the GFP and huCD2 IL-4 reporter mice 
previously available to this laboratory, disrupt the production of the reported gene. To ensure that the protein being reported remains available to influence immune responses in vivo, experiments are traditionally performed in heterozygous mice allowing protein production from the WT allele. However mice with only one WT IL-4 allele have been found to have deficiencies in Type 2 immune responses. A lack of one IL-4 allele decreases the amount of $\operatorname{IgE}$ generated in response to $\mathcal{N}$. brasiliensis infection by half ${ }^{31}$ and causes a ten-fold decrease in OVA-specific IgE in response to OVA/alum immunisation (Unpublished data Marcus Robinson).

IL-4 has been shown to be not required for the generation of Th2 cells ${ }^{31}$. However the role of this cytokine in Th2 expansion is less clear. Previous data by Van Panhuys et al. generated using heterozygous and homozygous knock-in reporter mice had been difficult to interpret because IL-4 is allelically expressed, with the majority of in vivo expression being mono-allelic. It was assumed that in the heterozygous GFP/IL-4 mice there would be a proportion of IL-4 expressing cells that were reporter negative while every Th2 cell induced to express IL-4 in the GFP/GFP mice would be $\mathrm{GFP}^{+}$. If IL-4 were dispensable for Th2 induction then in both the GFP/IL-4 and GFP/GFP mice the same total number of Th2 cells would be present. However because GFP would only mark the Th2 cells expressing from the reporter and not the WT allele in the GFP/IL-4 mice, these mice should have roughly half the number of reporter positive cells than the GFP/GFP mice. Due to the fact that both strains of mice had equal numbers of $\mathrm{GFP}^{+} \mathrm{CD}^{+} \mathrm{T}$ cells, I interpreted this to indicate that IL-4 present only in the heterozygous GFP/IL-4 mice expanded the total Th2 cell population. So while only half the potential IL-4 expressing cells would be reporting via GFP expression, the IL-4 driven expansion of the total Th2 cell population means that the number of $\mathrm{GFP}^{+}$cells would be comparable between the GFP/IL-4 and GFP/GFP strains ${ }^{31}$. Before this thesis no tools were available to independently measure IL-4 induction in IL-4 WT, heterozygous and knock-out mice.

A new transgenic IL-4 and IL-13 reporter system has been generated in an attempt to identify cells expressing IL-4 and IL-13 while avoiding the issues arising from the knock-in reporter systems that disrupt the parental alleles. In this chapter I will seek to characterise the new transgenic reporter mice, comparing them to current reporter system and other measures of IL-4 production. I will also investigate whether a third allele inserted transgenically into the genome is under allelic regulation as the parental genes are. Finally using the new reporter system as a read out of Th2 cell generation, the role of IL-4 for $i v$ vivo Th2 cell generation and expansion will be measured. 


\subsubsection{Aims}

The following experiments characterised the mechanisms that regulate IL-4 expression. To do this I validated and used multiple IL-4 reporter models to identify which IL-4 alleles $\mathrm{CD} 4^{+} \mathrm{T}$ cell were producing from. In vivo and in vitro models were used to induce $\mathrm{CD}^{+} \mathrm{T}$ cell IL-4 production, and huCD2, GFP and AmCyan IL-4 reporter expression measured and compared to assess the allelic regulation, and requirements for IL-4 on the expansion of Th2 cells. Specifically these experiments aimed to:

- Compare the three IL-4 reporter systems and measure their effectiveness for identifying IL-4 production

- Evaluate conditions which affect mono- and bi-allelic IL-4 expression

- Assess if clones maintain the same allelic phenotype

- Measure the impact of partial and total IL-4 deficiency on in vivo Th2 induction.

\subsection{Results}

\subsubsection{Analysis of IL-4 and IL-13 expression validates the 4C13R reporter mice}

The 4C13R reporter mouse reports IL-4 and IL-13 expression through the production of the fluorescent molecules AmCyan and DS-Red respectively. These transgenic reporter mice were created to avoid significantly affecting the expression of the WT IL-4 and IL13 alleles. To test this 4C13R splenocytes were stimulated in Th2 differentiating conditions, rested and restimulated. Stimulation was achieved by plating cells on $\alpha \mathrm{CD} 3$ coated plates, in the presence of IL-2 and IL-4 to drive Th2 differentiation. The cells were plated in media containing IL-2 but on uncoated plates to rest. During the $\alpha \mathrm{CD} 3$ driven restimulation no IL-4 was added allowing measurement of the IL-4 secreted by the cells during the 12 hours of culture with aCD3. Antibodies against the IL-4Ra (CD124) were added to the culture to inhibit receptor mediated uptake of IL-4. The proportions of $\mathrm{CD}^{+} \mathrm{T}$ cells that were positive for IL-4 and IL-13 via intracellular cytokine staining (ICS) were also compared after 6 hours of restimulation (Figure 3.1a). The proportion of $\mathrm{CD}^{+} \mathrm{T}$ cells that were positive for ICS IL-4 and IL-13 were found to be lower in cells from 4G13R compared with WT (Figure 3.1b). When the amounts of IL-4 released by restimulated cells from WT and 4C13R mice were compared by ELISA, a trend of 
slightly less cytokine in the 4C13R cultures was observed, but never a statistically significant difference (Figure 3.1c).

A very important readout of in vivo IL-4 production is IgE antibody class switching, so I tested that the $4 \mathrm{C} 13 \mathrm{R}$ reporter mice did not have deficiencies in $\mathrm{IgE}$ that had been observed in the other IL-4 knock-in reporter mice ${ }^{31}$. To test this, mice were infected subcutaneously with 600 L3 $\mathcal{N}$. brasiliensis, they were bled via the tail vein at the indicated days post infection and the serum levels of IgE assessed via ELISA. As had been seen with in vitro IL-4 production, lower IgE serum levels were observed in 4C13R mice compared with WT but the differences were statistically insignificant (Figure 3.1d).

Some minor deficiencies in Type 2 associated signalling and effector functions were observed using the 4G13R transgenic reporter mice however these were not as significant as those reported in the other reporter models ${ }^{31}$. Based on this data $4 \mathrm{C} 13 \mathrm{R}$ reporter mice are able to mount reasonably normal Type 2 immune responses in terms of IL-4, IL-13 and IgE production, making them an optimal model for identifying IL-4 and IL-13 producing cells in an immune competent system. 

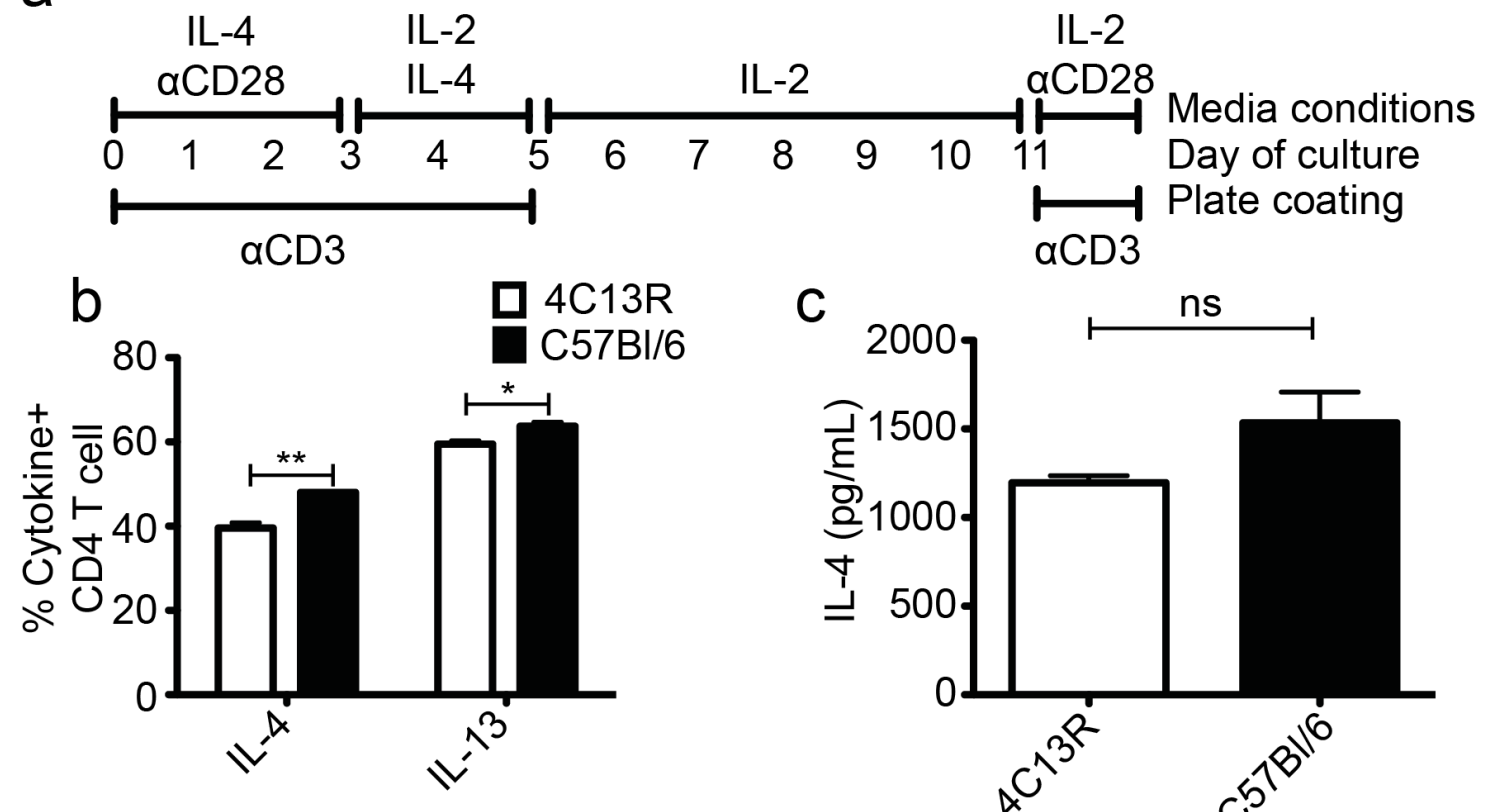

C
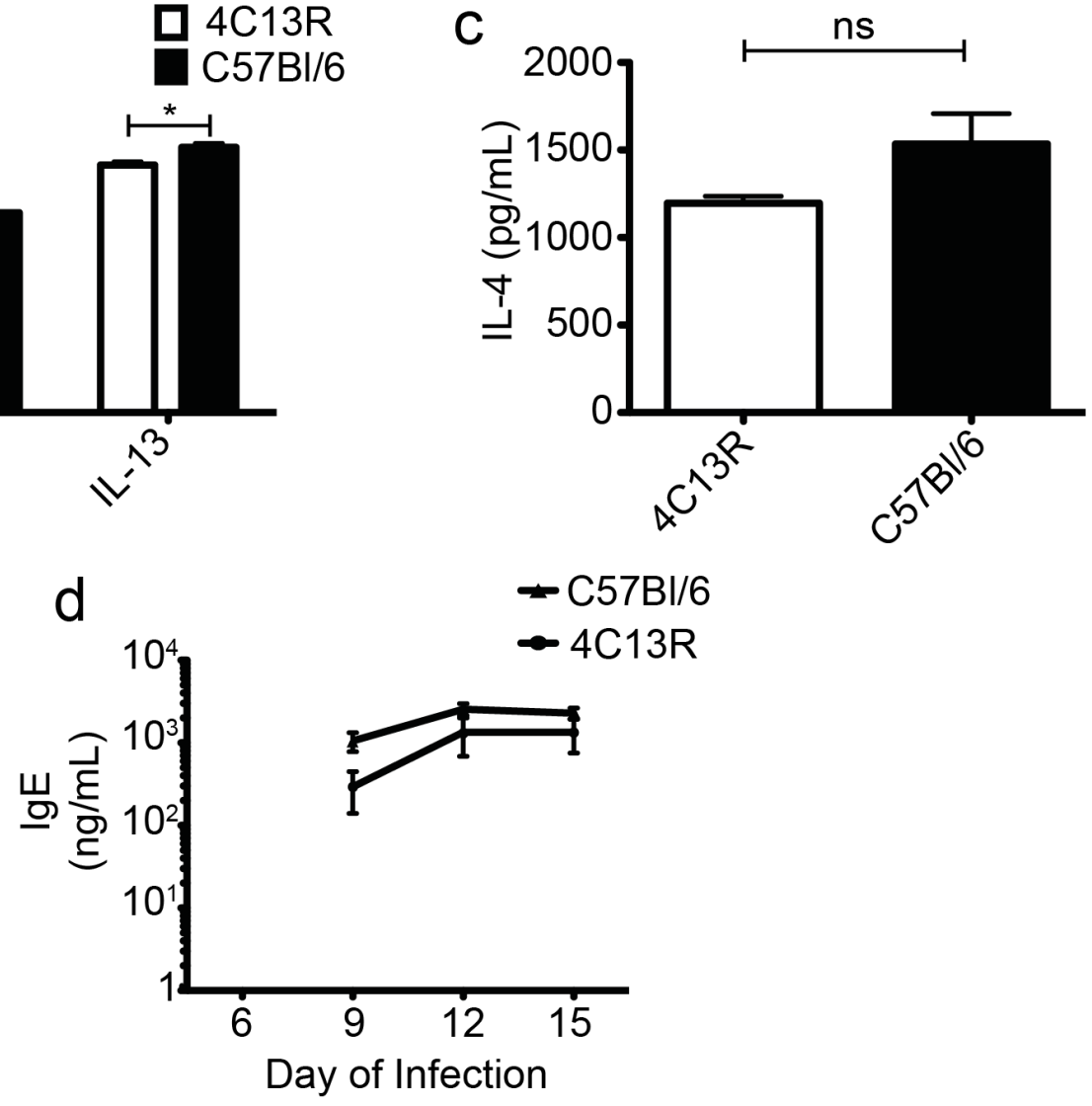

Figure 3.1 - 4C13R mice demonstrate only slight deficiencies in Type 2 cytokine production.

a. Schematic demonstrating Th2 culture system. Plates were coated with anti-CD3 (aCD3) antibodies and cells plated in Th2 media containing IL-4, IL-2 and anti-CD28 ( $\alpha$ CD28). On day 5 the cells were moved to uncoated plates and rested in IL-2. On day 11 cells were restimulated in $\alpha \mathrm{CD} 3$ coated plates in media with IL-2 and $\alpha \mathrm{CD} 28$. b. Cells from $4 \mathrm{C} 13 \mathrm{R}$ and $\mathrm{C} 57 \mathrm{Bl} / 6$ mice were cultured in the Th2 culture system. During the day 11 restimulation monensin was added to the cultures. Cells were harvested at 6 hours after restimulation and intracellularly stained for flow cytometric analysis of $\mathrm{CD}^{+} \mathrm{T}$ cell cytokine expression. Bar graphs show mean \pm S.E.M. $\mathrm{n}=3$ in each group. Data are representative of two independent experiments. Statistics were calculated for each cytokine using Student's t test * P $<0.05 * * \mathrm{P}<0.01$ c. Cells from 4C13R and C57Bl/6 mice were cultured in the Th2 culture system. During the restimulation $\alpha \mathrm{CD} 124$ antibodies were added to block IL-4 uptake and media was taken from the cells at 12 hours post restimulation and IL-4 levels were measured by ELISA. d. IgE titres from 4C13R and C57Bl/6 mouse serum at various time points after subcutaneous $\mathcal{N}$. brasiliensis infection. Plots show mean \pm S.E.M. $n=3$ in each group. Data are representative of two independent experiments. Statistics were calculated for each cytokine using a two way ANOVA with Bonferroni multiple comparison post test. Bar graphs show mean \pm S.E.M. $n=3$ in each group. Data are representative of two independent experiments. Statistics were calculated for each cytokine using Student's t test ns $=$ non significant. 


\begin{tabular}{|l|l|l|l|}
\hline Reporter Strain & $\begin{array}{l}\text { Number of } \\
\text { IL-4 alleles }\end{array}$ & Parental IL-4 alleles & Transgene \\
\hline 4G13R & 3 & IL-4/IL-4 & AmCyan \\
\hline GFP/IL-4 & 2 & GFP/IL-4 & None \\
\hline huCD2/IL-4 & 2 & huCD2/IL-4 & None \\
\hline huCD2/huCD2 & 2 & huCD2/huCD2 & None \\
\hline huCD2/GFP & 2 & huCD2/GFP & None \\
\hline 4G13RxhuCD2/huCD2 & 3 & huCD2/huCD2 & AmCyan \\
\hline 4G13RxGFP/GFP & 3 & GFP/GFP & AmCyan \\
\hline 4C13RxhuCD2/GFP & 3 & huCD2/GFP & AmCyan \\
\hline
\end{tabular}

Table 3-1 - Genotypes of IL-4 reporter mice strains

Summary table indicating the status of IL-4 alleles in the IL-4 reporter mice used in this chapter.

\subsubsection{The different IL-4 reporter mice can be independently used to identify allelic expression by CD4 $\mathrm{T}$ cells}

In order to measure the relative reporting kinetics by $\mathrm{CD} 4^{+} \mathrm{T}$ cells from established IL-4 reporter strains, the GFP and huCD2 knock-in reporter mice and the new transgenic 4C13R reporter strain, primary Th2 cultured and restimulated splenocytes were analysed for reporter expression. Splenocytes from IL-4 heterozygous huCD2/IL-4 and GFP/IL-4, and IL-4 deficient huCD2/huCD2 and huCD2/GFP were stimulated in the Th2 culture system (Figure 3.2a). All three of the reporter systems displayed similar induction kinetics for IL-4 reporting, with reporter positive cells being observed 2 days after plating on $\alpha \mathrm{CD} 3$ and peak production being reached on day 3 or 4 of culture. The resolution phases were also very similar, with GFP and huCD2 dropping to baseline on day 11 just prior to restimulation, while AmCyan contracted faster, dropping to baseline by day7 (Figure 3.2b). A far greater percentage of $\mathrm{CD} 4^{+} \mathrm{T}$ cells expressed huCD2 than GFP or AmCyan, raising some concern that there may be some preferential expression of the allele carrying huCD2 over the IL-4 or other reporter allele. However when the reporter expression in the double reporter $\mathrm{CD}^{+}$cells that carried both the huCD2 allele (Figure 3.2c) and the GFP allele was measured (Figure 3.2d), it was found that expression of each of these alleles were nearly identical in these cells compared to cells that only carried one of each of the alleles. Therefore while the alleles were expressed at different levels across the 
$\mathrm{CD}^{+} \mathrm{T}$ cell population the presence and expression of huCD2 did not effect the expression of the GFP allele and vice versa. 
a

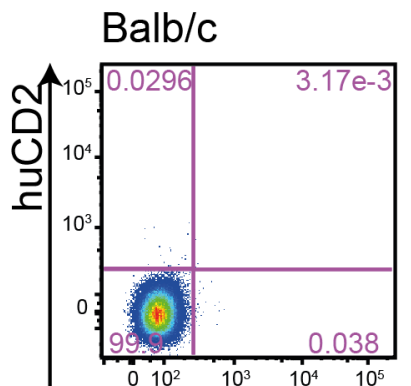

GFP/IL-4

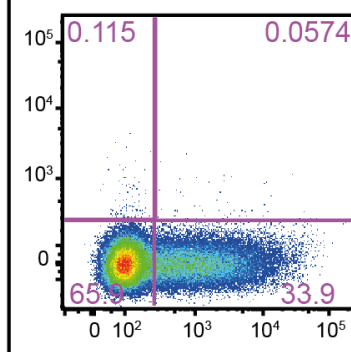

huCD2/IL-4

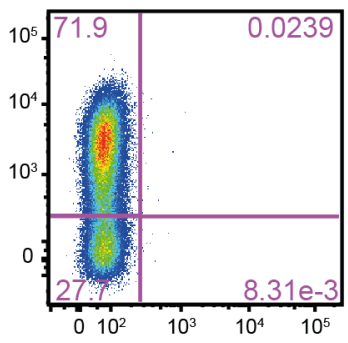

huCD2/huCD2

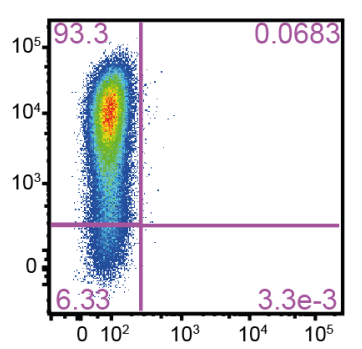

$4 C 13 R$

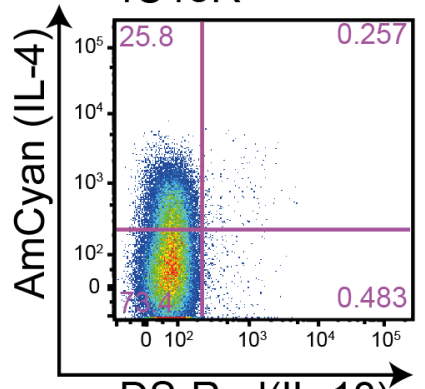

huCD2/GFP DS-Red(IL-13)

b
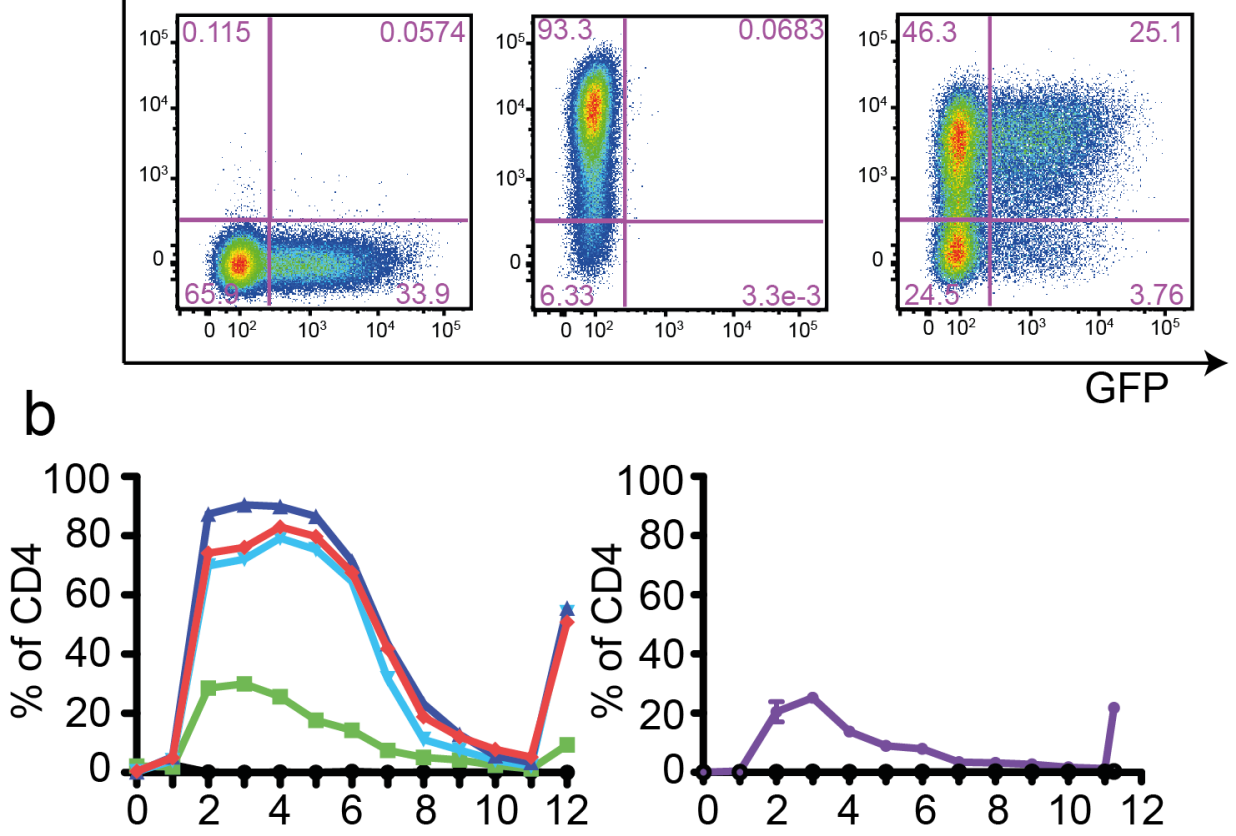

C
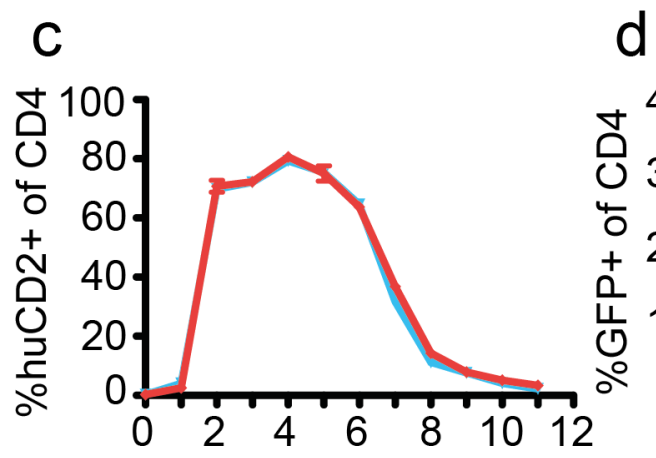

d

Figure 3.2 - 4G13R IL-4 reporting displays similar kinetics to knock-in reporter mice in vitro.

a. huCD2 and GFP expression by splenic $\mathrm{CD}^{+} \mathrm{T}$ cells from Balb/c, huCD2/IL-4 mice, GFP/IL-4 and huCD2/huCD2 and 4C13R mice at day 3 of the Th2 culture system. Plots are representative of triplicates and more than 4 experiments b. Proportion of $\mathrm{CD}^{+} \mathrm{T}$ cells expressing reporters from the indicated reporter mouse strains throughout the full time course of the Th2 culture system including 12 hour restimulation at day 11 . Plots show mean and S.E.M. $\mathrm{n}=3$ in each group. Data are representative of two independent experiments. c. Proportion of $\mathrm{CD}^{+} \mathrm{T}$ cells expressing huCD2 from the huCD2/IL-4 and huCD2/GFP reporter mouse strains throughout the time course of the Th2 culture system. Plots show mean and S.E.M. $\mathrm{n}=3$ in each group. Data are representative of two independent experiments. d. Proportion of $\mathrm{CD}^{+}{ }^{+} \mathrm{T}$ cells expressing GFP from the GFP/IL-4 and 
huCD2/GFP reporter mouse strains throughout the time course of the Th2 culture system. Plots show mean and S.E.M. $\mathrm{n}=3$ in each group. Data are representative of two independent experiments.

$\mathrm{CD}^{+} \mathrm{T}$ cells from the huCD2/GFP mice stimulated on $\alpha \mathrm{CD} 3$ in the presence of Th2 polarising media presented a consistent pattern of reporter expression, with cells expressing a singular reporter allele (mono-allelic) or both huCD2 and GFP alleles (biallelic) (Figure 3.3a). This expression profile was also seen when an IL-4 extracellular capture reagent was used on stimulated, IL-4 heterozygous, huCD2/IL-4 cells. Extracellular capture reagents are dual antibody reagents, an IL-4 specific antibody, bound via a biotin linker to a CD45 binding antibody. The CD45 antibody binds the capture construct to the haematopoietic cells, and the $\alpha$ IL- 4 antibody collects any IL-4 secreted by the bound cell These cells can then be probed with another IL-4 binding antibody conjugated to a fluorescent tag which can be detected by flow cytometry ${ }^{303}$. Using this tool with the huCD2/IL-4 cells, mono and bi-allelic IL-4 expression could be detected (Figure 3.3b). As had been seen in the huCD2/GFP cells, the proportion CD4 ${ }^{+}$ $\mathrm{T}$ cells expressing huCD2 was much higher than the proportion marked by the IL-4 capture reagent. However, unlike GFP, the IL-4 capture reagent positive cells were more evenly distributed between the mono-allelic and bi-allelic expressing populations. Intracellular staining for IL-4 ${ }^{+} \mathrm{CD} 4^{+} \mathrm{T}$ cells from splenocyte cultures restimulated for 6 hours on day 11 of the stimulation and rest culture system demonstrated further the allelic expression of IL-4. The IL-4 heterozygous mice, huCD2/IL-4 and GFP/IL-4 had less than half the proportion of IL- $4^{+} \mathrm{CD} 4^{+} \mathrm{T}$ cells compared with WT cells, while the homozygous knock-out reporters huCD2/huCD2 and huCD2/GFP had no IL-4 (Figure 3.3c). ELISAs of supernatants from cultures restimulated for 12 hours also demonstrated decreased levels of secreted IL-4 from the heterozygous cells (Figure 3.3d).

These data demonstrate that IL-4 is expressed allelically and this can be detected via the use of reporter systems, and while the reporters are expressed independently of each other the relative reporting frequencies of each reporter are different. Due to the allelic nature of IL-4 expression, the replacement of an IL-4 allele by a reporter means less total IL-4 is produced and there are fewer IL-4 producing cells, as some are expressing the reporter construct rather than wild type IL-4. 
a
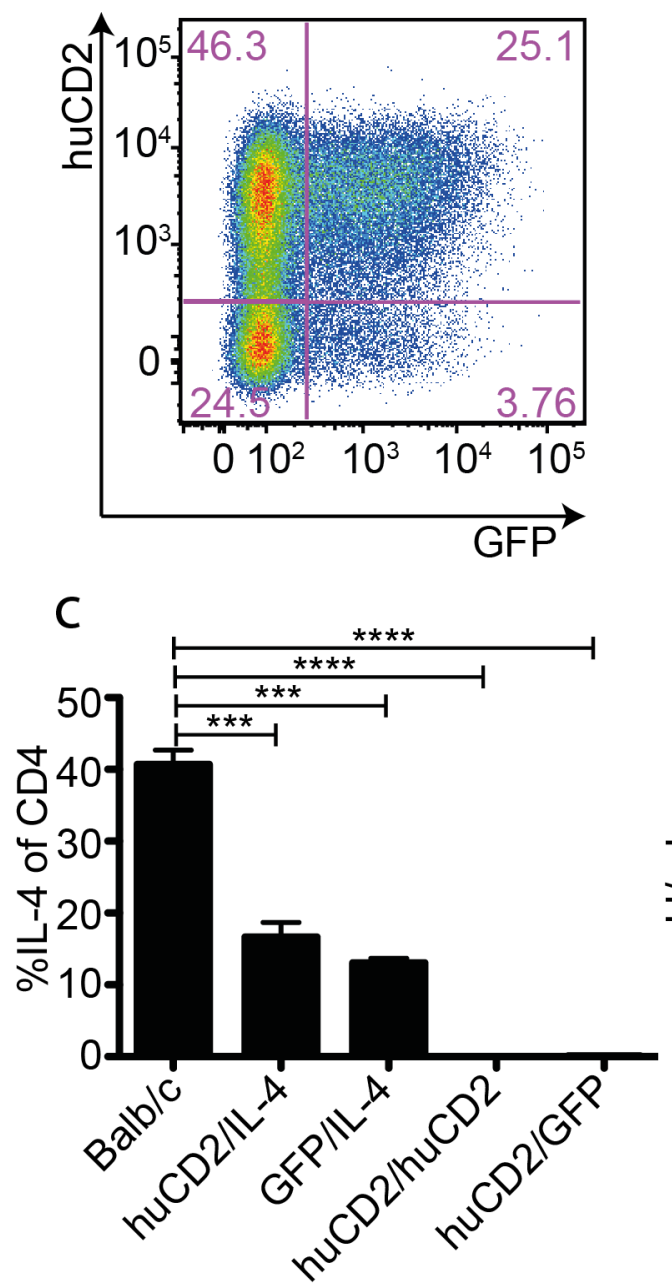

b

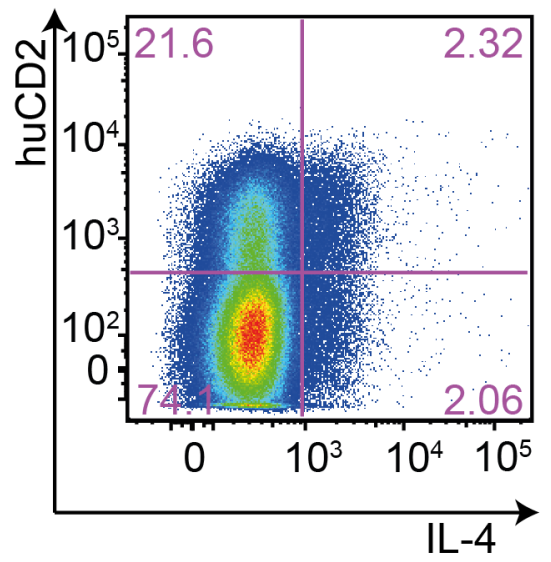

d

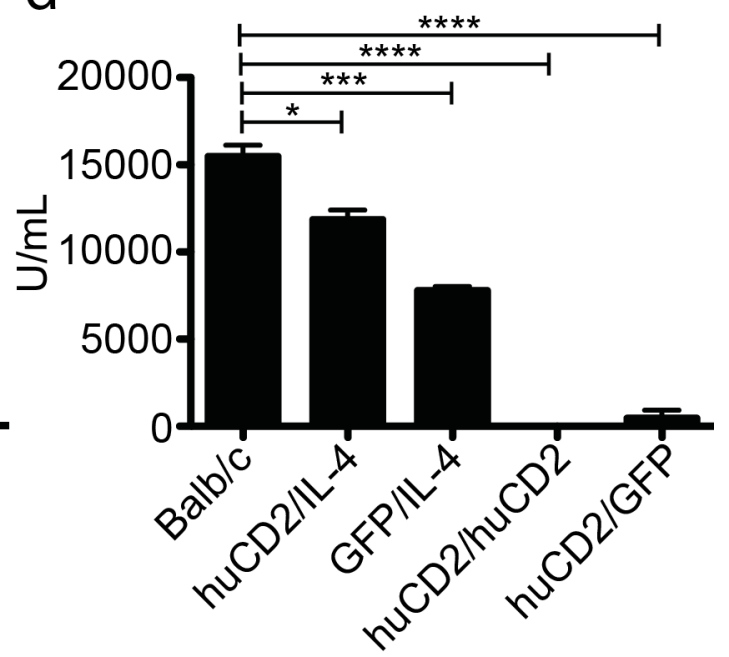

Figure 3.3 - IL-4 expression is under allelic control.

a. Reporter expression by huCD2/GFP CD4+ $\mathrm{T}$ cells cultured for 3 days in the Th2 culture system. Plot is representative of greater than five experiments b. huCD2 and IL-4 expression by huCD2/IL-4 CD4 ${ }^{+} \mathrm{T}$ cells assessed by use of the IL-4 extracellular capture reagent. Plot is representative of $n=3$ from two independent experiments. c. Proportion of IL-4 secreting CD4 ${ }^{+} \mathrm{T}$ cells assessed by ELISPOT. Cells were harvested at day 10 of the Th2 culture and restimulated in ELISPOT plates coated with aIL-4 and aCD3 antibodies. Plots show mean and S.E.M. $n=2$ in each group. Data are representative of two independent experiments. Statistics were calculated using One-way ANOVA with Bonferroni's multiple comparisons post-test $* \mathrm{P}<0.05 * * \mathrm{P}<0.01$ *** $\mathrm{P}<0.001 * * * * \mathrm{P}<0.0001$ d. Concentration of IL-4 in the media after 12 hours of restimulation on day 11 of the Th2 culture. Plots show mean and S.E.M. $n=2$ in each group. Data are representative of two independent experiments. Statistics were calculated using One-way ANOVA with Bonferroni's multiple comparisons post-test $* \mathrm{P}<0.05 * * \mathrm{P}<0.01 * * * \mathrm{P}<0.001$ $* * * * \mathrm{P}<0.0001$

\subsection{3 $\mathrm{T}$ cell receptor stimulation affects IL-4 allelic expression profiles by $\mathrm{CD}^{+} \mathrm{T}$ cells}

I utilised the Th2 inducing culture system to evaluate the mechanisms that controlled the allelic expression pattern of $\mathrm{IL}-4$ by $\mathrm{CD}^{+} \mathrm{T}$ cells. $\mathrm{T}$ cells receive several critical 
differentiation driving signals once in culture, predominantly through their TCR interacting with the plate bound $\alpha \mathrm{CD} 3$, with secondary signals through co-stimulation with $\alpha$ CD28 and finally IL-4 and IL-2 acting through their cognate receptors driving differentiation towards a Th2 phenotype. Cultures were set up to test which signals were controlling the $\mathrm{CD}^{+} \mathrm{T}$ cell differentiation into Th2, and if any of these particular signals preferentially drive bi-allelic as opposed to mono-allelic expression. Previous work has demonstrated the requirements for IL-4 ${ }^{30}$ and IL-2222 for optimal Th2 differentiation in vitro, so Th2 cultures were assessed on day 3 to test the effects of feeding with additional cytokines and/or co-stimulation added to the culture on days 1 and 2 after plating. It was found that the allelic expression pattern of the $\mathrm{CD} 4^{+} \mathrm{T}$ cells was totally unaffected by the addition of cytokines after the first day of plating, even in the unfed group, where half of the culture volume was replaced with media without stimulatory cytokines or antibodies daily (Figure 3.4). From this data it can be deduced that cells either receive all the signals they require for full Th2 differentiation and set allelic expression during the first day of culture, or the amount of cytokines and antibodies used maximally drove Th2 differentiation and the allelic pattern I observed was what this maximal stimulation would always induce. 


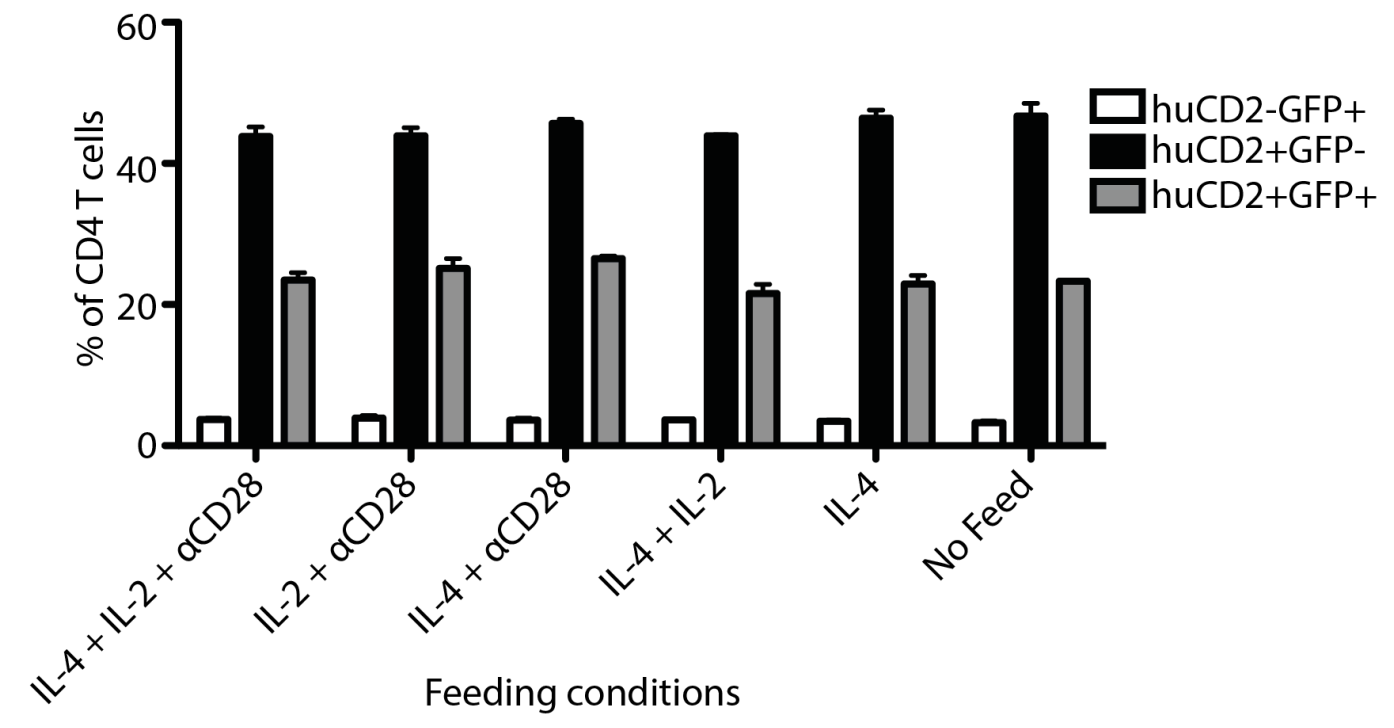

Figure 3.4 - IL-4 allelic expression patterns are unaffected by feeding regime.

a. Reporter expression by huCD2/GFP cells 3 days after plating onto aCD3 in Th2 media. On days 1 and 2 half the media was removed and replaced with complete media containing the indicated cytokine and antibody mixes. Bar graph show mean and S.E.M. $n=2$ in each group. Data are representative of two independent experiments. Statistics were calculated individually for each strain using One-way ANOVA with Bonferroni's multiple comparisons post-test with no significant difference. 
To determine the role of TCR activation on allelic expression, a titration of the concentration of $\alpha \mathrm{CD} 3$ that coated the stimulation plates was undertaken, limiting the amount of stimulation the $\mathrm{CD} 4^{+} \mathrm{T}$ cells received through their TCRs. When cultured in differentiation neutral conditions (Th0), with antibodies to neutralise the polarising cytokines IL-4 and IFN- $\gamma$, there was very little reporter expression detected at any $\alpha \mathrm{CD} 3$ concentration (Figure 3.5a). Of the positive cells there were almost no bi-allelic cells, and even proportions of mono-allelic huCD2 and GFP positive cells (Figure 3.5b). The concentration of $\alpha \mathrm{CD} 3$ had a profound effect on IL-4 reporting by CD4+ $\mathrm{T}$ cells cultured in Th2 conditions, with an increase in reporter expressing cells correlating with increasing $\alpha \mathrm{CD} 3$ concentrations (Figure 3.5a). The proportion of reporter bi-allelism increased with the amount of TCR stimulation, with an associated decrease in the proportion of monoallelic $\mathrm{GFP}^{+}$. Of the reporter expressing $\mathrm{CD}^{+} \mathrm{T}$ cells there was no change in the proportion of mono-allelic huCD2 ${ }^{+}$cells across the concentration range of $\alpha \mathrm{CD} 3$ (Figure 3.5b). Therefore bi-allelic reporting increases with increased stimulation through the TCR, and the observed decrease in mono-allelic $\mathrm{GFP}^{+}$indicates that as the $\alpha \mathrm{CD} 3$ concentration increases, the majority of $\mathrm{CD} 4^{+} \mathrm{T}$ cells expressing this allele are expressing IL-4 using both alleles. 
a

Unstimulated $\quad 0.025 \mu \mathrm{g} / \mathrm{mL} \quad 0.125 \mu \mathrm{g} / \mathrm{mL} \quad 0.25 \mu \mathrm{g} / \mathrm{mL} \quad 0.5 \mu \mathrm{g} / \mathrm{mL} \quad 1 \mu \mathrm{g} / \mathrm{mL}$ Th0 Media
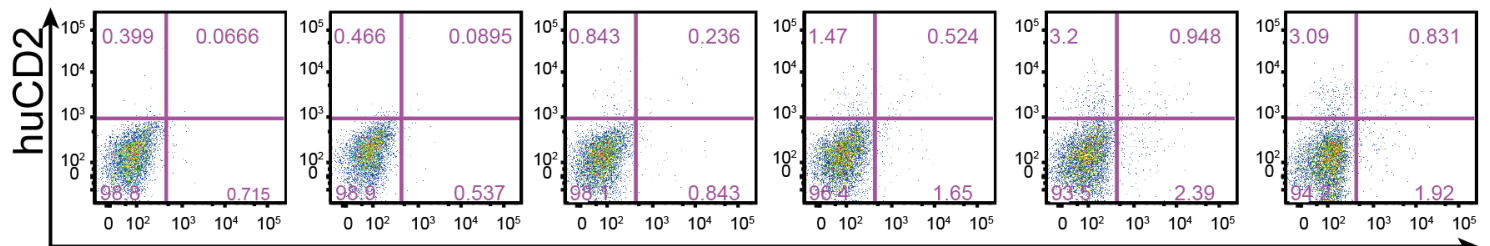

Th2 Media
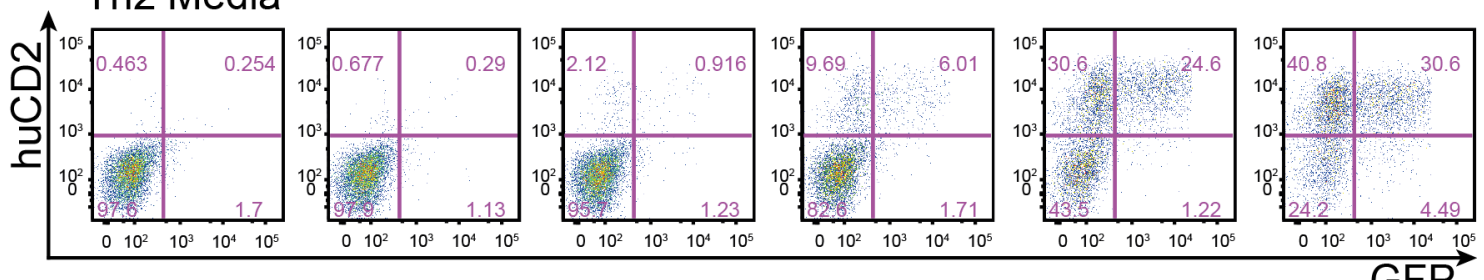

b

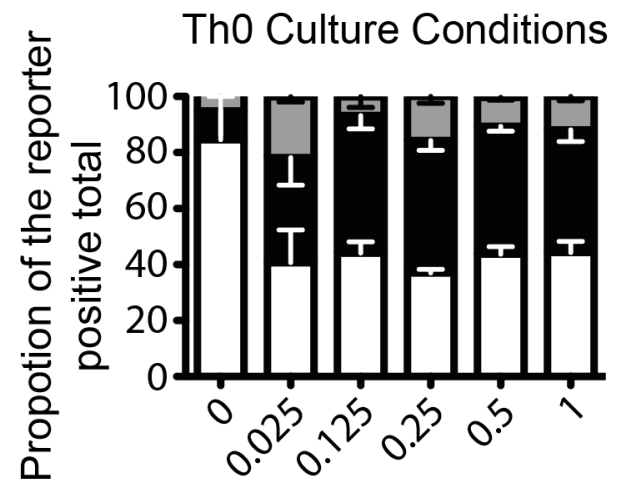

Th2 Culture Conditions

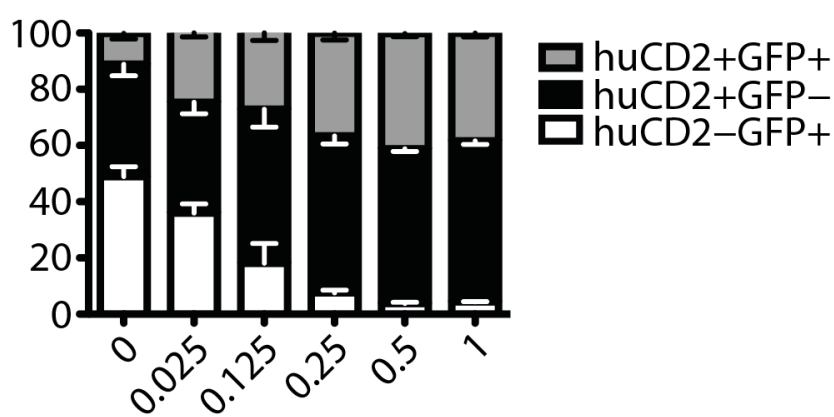

aCD3 Concentration

Figure 3.5 - Anti-CD3 concentration affects reporter expression and bi-allelism.

a. and b. Representative plots and graphs of the proportion of reporter expression by huCD2/GFP cells from plates coated with a range of aCD3 concentrations, indicated above, at day 3 of the Th2 culture or Th0 culture systems. For Th0 cultures, cells were plated in media containing IL-2 and aCD28, aIL-4 and aIFN- $\gamma$ antibodies in the media. Bar graphs show mean and S.E.M. $\mathrm{n}=3$ in each group. Data are representative of two independent experiments. 
The scarcity of mono-allelic $\mathrm{GFP}^{+} \mathrm{CD}^{+} \mathrm{T}$ cells observed in cultures with relatively high levels of overall reporter expression led to further investigation of the specificities of the IL-4 reporter systems. While green fluorescent protein is expressed intracellularly, the huCD2 reporter in translocated to the cell surface, allowing the reporter-positive cells to be identified by staining with fluorescently tagged monoclonal antibodies. As the reporterpositive cells are expressing the huCD2 molecules on their surface I hypothesised that the protein could be transferred to other cells not expressing the reporter allele themselves. To test this huCD2 IL-4 reporting splenocytes were cultured with and without carboxyfluorescein succimidyl ester (CFSE) labelled wild-type (WT) splenocytes in Th2 conditions. Two days after beginning the culture, cells were harvested and stained with the full antibody cocktail including the huCD2 specific antibody. Doublets were excluded and gates set based upon the background of stained huCD2 and GFSE labelled WT $\mathrm{CD}^{+} \mathrm{T}$ cells cultured alone. A small percentage of CFSE labelled cells from the mixed culture that had acquired huCD2 could be detected (Figure 3.6a). This was not due to nonspecific staining as this population was missing from the single cultured cells that were stained with the same antibody cocktail.

To confirm that transfer of CFSE to huCD2 expressing cells was not occurring, the mixed culture system was repeated with cells that constitutively expressed intracellular enhanced GFP (eGFP). Again an appearance of huCD2 expression was detected on cells that did not carry the huCD2 allele (Figure 3.6b). The transfer of huCD2 was also observed between co-cultured cells from huCD2 expressing cells (on a Balb/c background) and C57Bl/6 cells, identified by their differing MHC class II haplotypes (data not shown). It appeared that the transfer of huCD2 could occur within a short period of time. In one experiment huCD2 expressing and eGFP cells, that had been cultured individually, were mixed just prior to staining and a population of eGFP and huCD2 positive cells was detected, though at roughly half the proportion compared to cells that were cultured together for the 2 days (data not shown). Therefore huCD2 can transfer to cells which are not actively expressing the huCD2 allele, which can partially explain why the huCD2 reporter appears to mark such a high percentage of CD4 T cells in Th2 culture, and why there is such a preponderance for $\mathrm{GFP}^{+}$cells to be bi-allelic from cultured huCD2/GFP splenocytes. However, at most, only 5\% of the non-huCD2 carrying cells were observed becoming positive for huCD2, so transfer of the reporter protein must be considered but does not completely explain the much higher proportions of huCD2 expressing cells compared with the other reporter systems. 

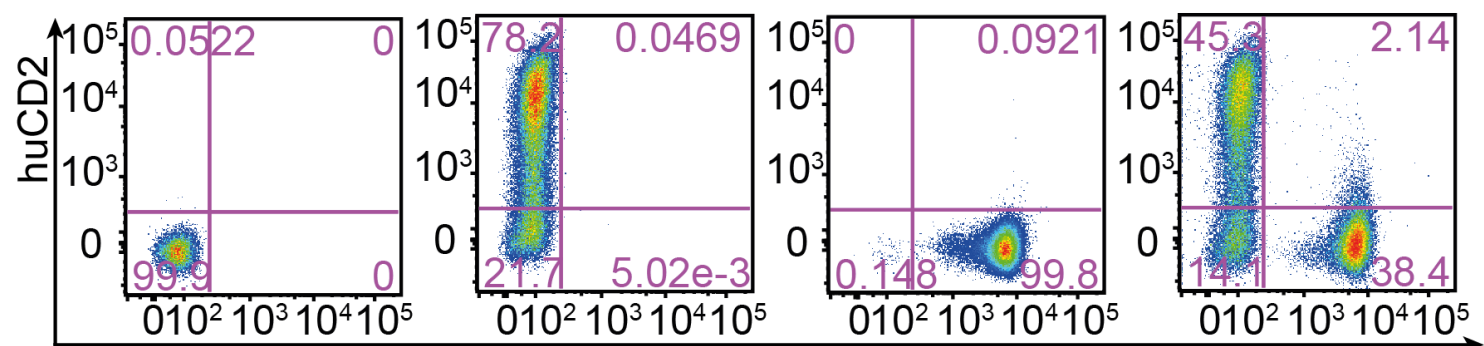

CFSE

b
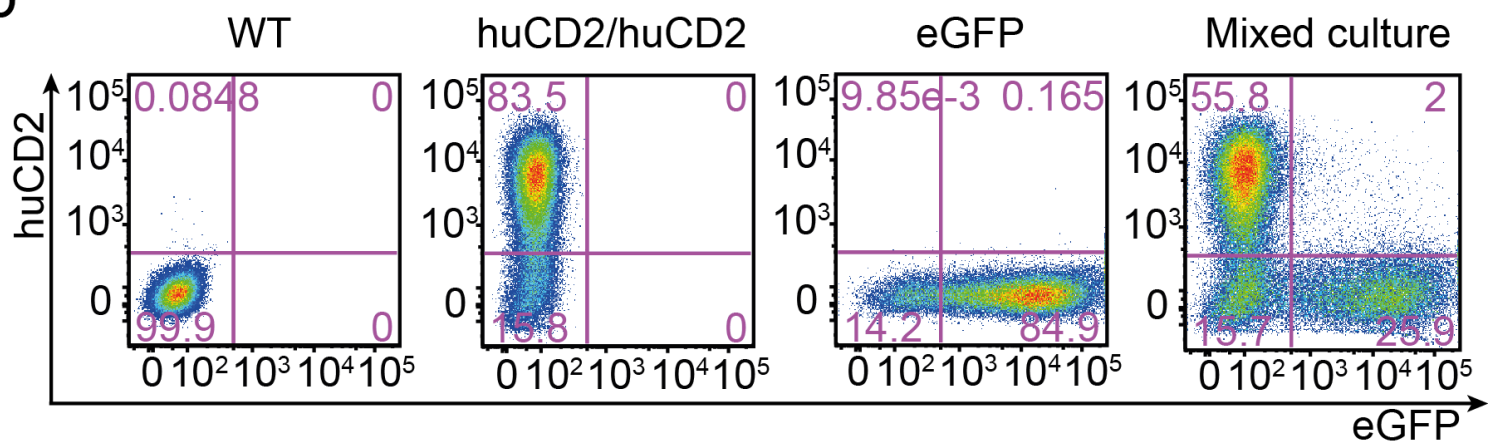

Figure 3.6 - huGD2 can be transferred between cells in culture.

a. huCD2 expression on WT splenocytes labelled with CFSE and unlabelled huCD2/huCD2 splenocytes cultured for 2 days in the Th2 system either by individually or mixed together. Plots representative of triplicates from two independent experiments. b. huCD2 expression on eGFP splenocytes and huCD2/huCD2 splenocytes cultured for 3 days in the Th2 system either by individually or mixed together. Plots representative of triplicates from two independent experiments. 


\subsubsection{Allelic IL-4 expression patterns are not maintained by restimulated $\mathrm{CD}^{+} \mathrm{T}$ cells}

Utilising the double reporter mice allowed further research into the phenomena of allelic expression of IL-4 by Th2 cells. Mono-allelic expression could be explained by a restriction in available transcriptional machinery, limiting the alleles a cell can transcribe into mRNA. Alternatively the cells could actively silence alleles through epigenetic regulation and dense packing of the DNA at key sites along a particular allele ${ }^{304}$. If the cells were controlling their allelic expression by epigenetic changes it would be likely that the cells, and their progeny, would maintain their allelic profile, even after resting and restimulation. To test this theory huCD2/GFP splenocytes were stimulated for 5 days in Th2 conditions, then CD4 T cells were sorted based upon their reporter expression into four populations, reporter negative (huCD2-GFP-), GFP positive (huCD2-GFP ${ }^{+}$, huCD2 positive (huCD $\left.2{ }^{+} \mathrm{GFP}-\right)$ and double positive $\left(\right.$ huCD $\left.2{ }^{+} \mathrm{GFP}^{+}\right)$(Figure $\left.3.7 \mathrm{a}\right)$. The cells were then plated on uncoated plates in IL-2 supplemented media and rested them for 10 days, until they were no longer expressing detectable reporter, at which point they were restimulated for 6 hours and their reporter expression assessed (Figure 3.7b). Regardless of the reporter profile the cells expressed on day 5 of culture, after restimulation all populations demonstrated the same pattern of expression (Figure 3.7c). The only notable difference was that cells that were reporter negative at the time of sorting had a lower proportion of total reporter positive cells upon restimulation (Figure 3.7d). This indicated that clones of Th2 cells did not maintain a memory of previous allelic expression and the IL-4 allele that a clone expresses can be reset after rest and restimulation. 
a

Set up cultures

Sort

Rest cells in

Day 5

IL-2 media

Re-Stimulate

Day 0

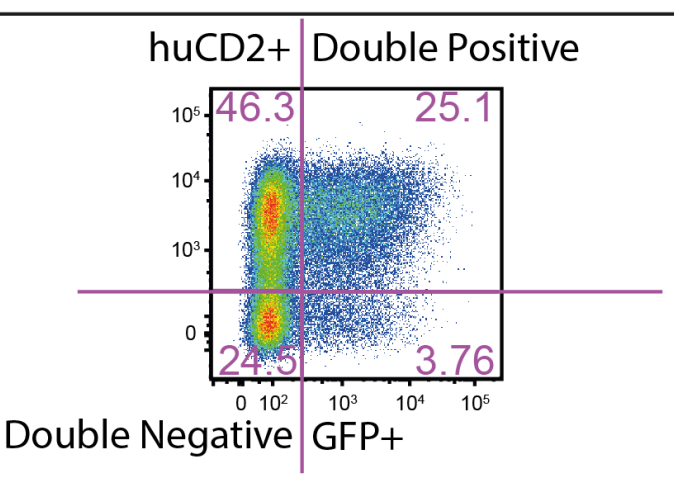

Day 17

\begin{tabular}{lr|r|r}
\hline $\begin{array}{l}\text { huCD2/GFP } \\
\text { splenocytes were }\end{array}$ & huCD2+ & Double Positive & $\begin{array}{r}\text { Sorted/Rested cells } \\
\text { plated on aCD3 } \\
\text { plated on aCD3 } \\
\text { coated plates in Th2 } \\
\text { conditions }\end{array}$ \\
IL-2 for 6 hours
\end{tabular}

b

huCD2+ Double Positive
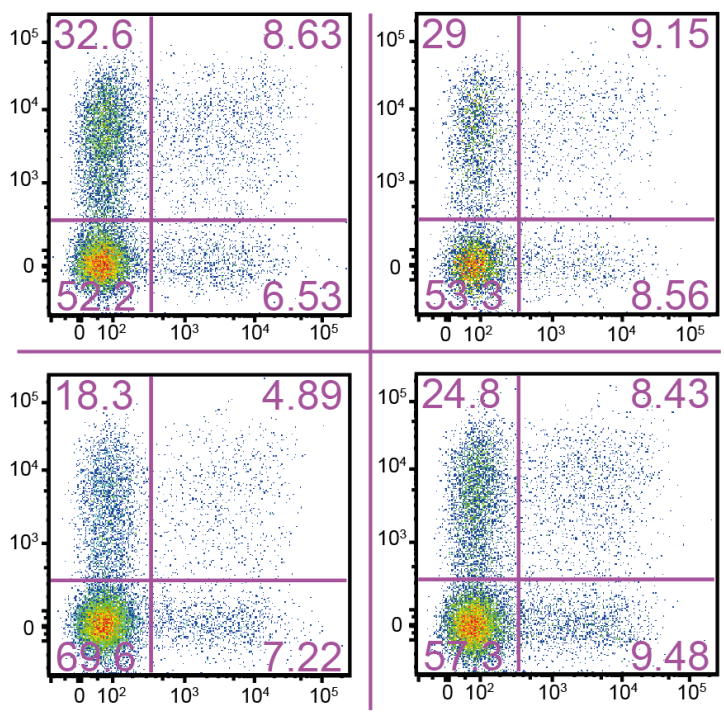

Double Negative

GFP+

C

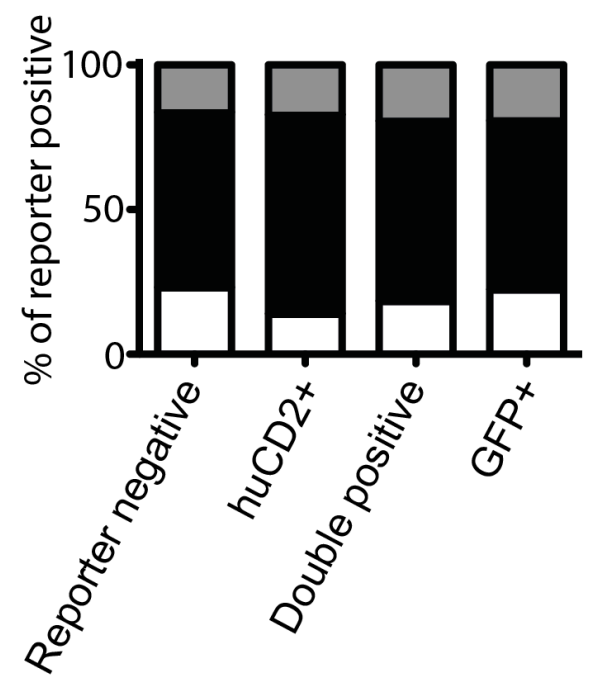

d

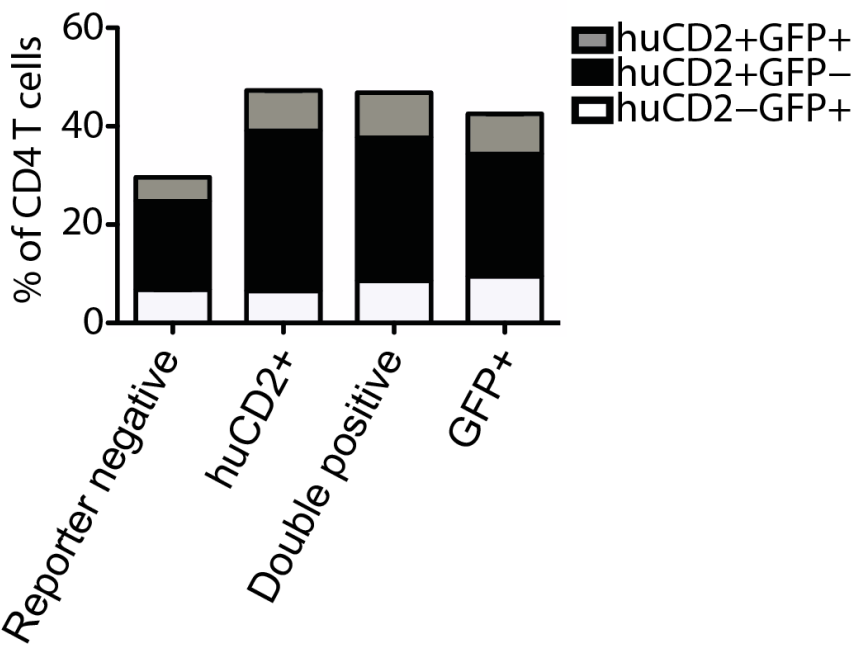


Figure 3.7 - Sorted populations do not retain their IL-4 allelic patterns upon restimulation.

a. Experimental schematic. Cells were cultured in the previously described Th2 system for 5 days then sorted into four $\mathrm{CD}^{+} \mathrm{T}$ cell populations based upon their reporter expression. The cells were then rested in uncoated plates with IL-2 containing media until day 17 when reporter expression had dropped to background. Cells were then restimulated for 12 hours and reporter expression analysed. b. Representative plots of reporter expression by the 4 restimulated populations. c. Proportions of restimulated $\mathrm{CD}^{+} \mathrm{T}$ cells expressing huCD2, GFP or both reporters. d. Proportion of the reporter expressing cells that are huCD2, GFP or dual reporter positive. Bar graphs show mean and S.E.M. $n=3$ in each group. All data is representative of two independent experiments.

\subsubsection{Expression of the 4C13R transgenic IL-4 reporter is allelically regulated}

The 4C13R reporter mice were created utilising transgenic technology with the intention that the endogenous parental IL-4 and IL-13 genes would remain untouched and available for normal cytokine transcription. However the uncontrolled insertion of the transgenic construct could mean that the transgenic alleles do not receive the same transcriptional enhancement, or regulation, as the parental alleles. Knowing that the expression of IL-4 is controlled allelically it remained to be seen if AmCyan, the transgenic IL-4 reporter, would also be under allelic restriction. If the transgenic allele had escaped regulation, then every cell that expressed IL-4 using either of the parental alleles would also express AmCyan. However if AmCyan was also under allelic regulation then a similar pattern of expression as the cells from the double knock-in reporter huCD2/GFP would be observed, with some cells expressing AmCyan, or a parental allele mono-allelically, and some expressing bi-allelically AmCyan and the parental IL-4 allele. To test if AmCyan was expressed allelically mice carrying the 4G13R construct were crossed with mice that were homozygous for either the huCD2 or GFP reporters on their parental IL-4 alleles. The resulting offspring carried the 4G13R construct, one WT parental IL-4 allele and one knock-in reporter allele, either huCD2 or GFP, making these mice triple allelic for the IL-4 locus. These reporter mice were then used to test the reporter expression by $\mathrm{CD} 4^{+} \mathrm{T}$ cells in both the Th2 culture, and the pTh2 assay, which involves intradermal HDM injection into the ears of IL-4 reporter mice and the reporter expression by $\mathrm{CD} 4{ }^{+} \mathrm{T}$ cells in the auricular draining $\mathrm{LN}$ assessed 7 days later.

When 4G13RxhuCD2/huCD2 $\mathrm{CD}^{+} \mathrm{T}$ cells were cultured in Th2 conditions the reporter pattern was remarkably similar to the huCD2/GFP cells. The majority of reporter positive $\mathrm{CD}^{+}{ }^{+} \mathrm{T}$ cell mono-allelically expressed huCD2 ${ }^{+}$, while almost all the $\mathrm{AmCyan}^{+}$cells were bi-allelic and also expressing huCD2. AmCyan ${ }^{+} \mathrm{CD}^{+} \mathrm{T}$ cells from 
Th2 cultured 4C13RxGFP/GFP were almost equally split between the mono-allelic and bi-allelic populations, with slightly more in the bi-allelic population (Figure 3.8a). House dust mite responsive $\mathrm{CD}^{+} \mathrm{T}$ cells from the pTh2 assay presented different expression patterns. The 4C13RxhuCD2/huCD2 cells had the majority of the reporter positive being mono-allelically AmCyan ${ }^{+}$, and an even proportion of mono-allelic huCD2 ${ }^{+}$, and bi-allelically expressing cells. Reporter expression by 4G13RxGFP/GFP CD4+ T cells in the pTh2 assay showed equal proportions of both the mono-allelic populations and the biallelically expressing cells (Figure 3.8b).

To assess the allelic expression of AmCyan against WT IL-4, the IL-4 extracellular capture reagent was used on Th2 cultured 4C13R CD4 ${ }^{+} \mathrm{T}$ cells. Th2 $\mathrm{CD} 4^{+} \mathrm{T}$ cells that were singularly expressing AmCyan or IL-4 could be detected alongside a population of bi-allelic Th2 cells (Figure 3.8c). As a control to test the specificity of the extracellular capture reagent Th2 cultured cells from IL-4 sufficient CD45.1 congenic mice were mixed with CD45.2 expressing IL-4 deficient cells. The populations could be distinguished based upon their congenic expression of CD45 so the mixed cells were treated with the IL-4 capture reagent and then analysed to check that only the IL-4 sufficient cells were marked positive by the reagent. The extracellular capture reagent was very specific, only staining the IL-4 sufficient CD45.1 ${ }^{+}$cells and not the IL-4 deficient cells (Figure 3.8d). These data show that the expression of AmCyan, despite being a transgenic reporter of IL-4, was also under allelic control. Moreover the expression of AmCyan more closely resembles the GFP reporter system than the huCD2 reporter. 

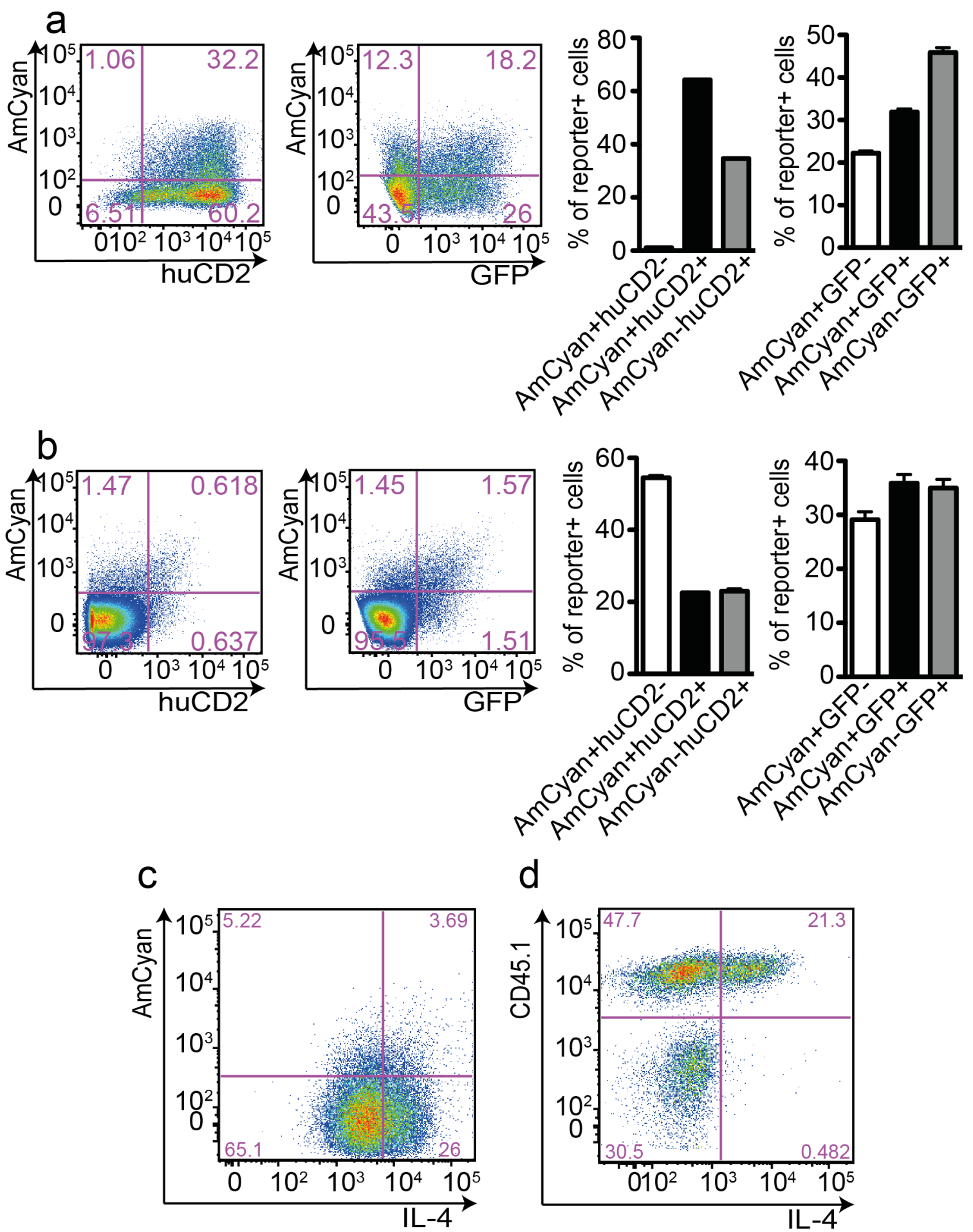

Figure 3.8 - Expression of the 4G13R transgenic IL-4 reporter is under allelic control.

a. Reporter expression by 4C13RxhuCD2/huCD2 and 4C13RxGFP/GFP CD4+ $\mathrm{T}$ cells on day 3 of the Th2 culture system. Bar graphs show mean and S.E.M. $n=3$ in each group. Plots are representative of two independent experiments b. Reporter expression by 4C13RxhuCD2/huCD2 and 4C13RxGFP/GFP CD4+ T cells from the draining LN on day 7 of the pTh2 assay. Bar graphs show mean and S.E.M. $n=3$ in each group. Plots are representative of two independent experiments. c. Representative plot of AmCyan and IL-4 expression by 4C13R CD4 ${ }^{+} \mathrm{T}$ cells on day 3 of the Th2 culture system assessed by use of the IL-4 extracellular capture reagent. d. Control experiment for IL-4 extracellular capture reagent, IL-4 sufficient CD45.1+ 
$\mathrm{CD}^{+} \mathrm{T}$ cells were cultured in the Th2 culture system with IL-4 deficient GD45.1- CD4+ $\mathrm{T}$ cells. All plots are representative of $n=3$ in each group. Plots are representative of two independent experiments

\subsubsection{Triple allele IL-4 reporter system indicates the special nature of bi-allelically expressing $\mathrm{CD4}^{+} \mathrm{T}$ cells}

Three independent reporters of IL-4 expression were available, two knocked into the parental IL-4 alleles and one transgenic reporter. A cross of all three of the reporter systems was established to test what effect a third allele would have on the generation of mono and bi-allelically expressing $\mathrm{CD}^{+}{ }^{+} \mathrm{T}$ cells. Splenocytes from triple reporter mice were stimulated in the Th2 culture system and the mice were also subjected to the pTh2 assay. $\mathrm{CD}^{+} \mathrm{T}$ cells were identified and four populations defined by their expression of two of the IL-4 reporters were identified. These populations were defined as reporter negative, single reporter positive or double positive. The expression of the third IL-4 reporter was then assessed from each of these four populations. In both assays cells expressing two of the reporter alleles (double positive) were more likely to express a third allele regardless of which reporters were assessed (Figure 3.9). The only point of note was that the AmCyan-GFP+ ${ }^{+}$cells were just as likely to express huCD2 as the $\mathrm{AmCyan}^{+} \mathrm{GFP}^{+}$ double positive cells. These double positive cells also had a higher median fluorescent index (MFI) for the third reporter compared to the singular or reporter negative populations (Figure 3.9). From these data bi-allelic expressing cells were more likely, and more capable of utilising a third IL-4 allele. 
a
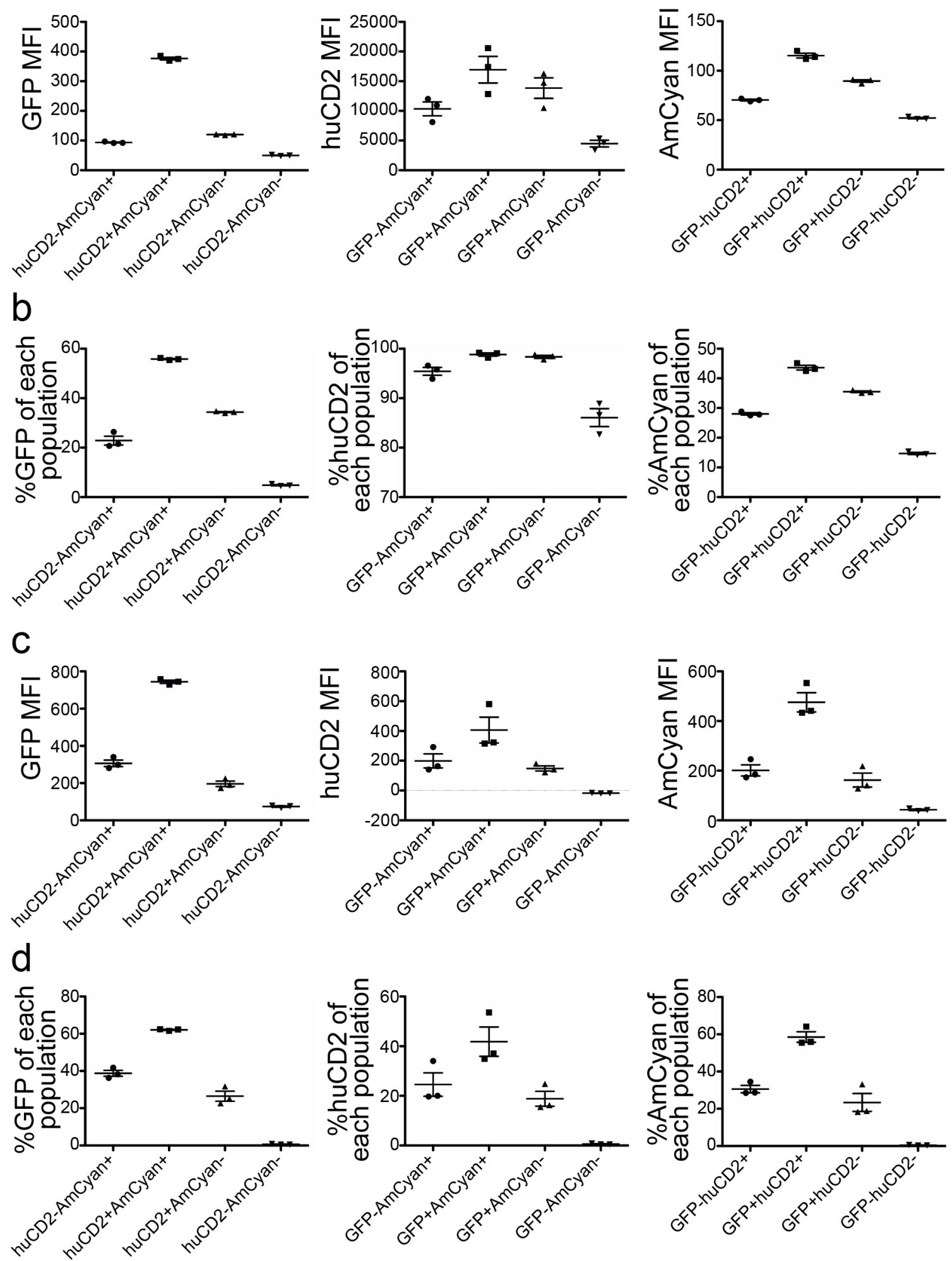

Figure 3.9 - Qualities of multi-allelically IL-4 expressing cells elucidated by use of triple reporter mice.

(a. b.) Reporter expression of CD4+ $\mathrm{T}$ cells from 4C13RxhuCD2/GFP reporter mice at day 3 of the Th2 culture system. CD4 ${ }^{+} \mathrm{T}$ cells were defined into 4 populations using 2 of the reporters and then $\mathbf{a}$. the MFI and $\mathbf{b}$. the proportion expressing the third reporter was analysed for each population. (c. d.) Reporter expression of draining LN CD4+ T cells from 4G13RxhuCD2/GFP reporter mice at day 7 of the pTh2 assay. CD4 ${ }^{+} \mathrm{T}$ cells were defined into 4 populations using 2 of 
the reporters and then c. the MFI and d. the proportion expressing the third reporter was analysed for each population.

\subsubsection{IL-4 is not important for the expansion of Th2 cells in vivo}

The transgenic 4G13R reporter mice enabled measurement of the induction of IL-4 expressing Th2 cells independently of the parental alleles. Crossing these mice to GFP IL-4 reporter strains allowed the generation of reporter mice that were heterozygous deficient (4C13RxGFP/IL-4) and homozygous IL-4 deficient (4C13RxGFP/GFP) as well as the original IL-4 WT (4C13R) strain. Using AmCyan as an independent read out of IL-4 producing Th2 cells, these strains of mice allowed the effect of decreased IL-4 availability on the expansion of Th2 cells to be assessed. The various strains of mice were challenged with house dust mite via the pTh2 model and the AmCyan expression by the dLN CD4 ${ }^{+} \mathrm{T}$ cells assessed on day 7 after intradermal injection.

The percentage of $\mathrm{CD}^{+} \mathrm{T}$ cells that expressed AmCyan appeared to titrate with the availability of WT IL-4 alleles; significant difference was observed between the WT 4C13R strain and both the heterozygous an homozygous deficient strains, but the difference between the 4C13RxGFP/GFP and 4C13RxGFP/IL-4 strains was not significant (Figure 3.10a). The numbers of AmCyan ${ }^{+} \mathrm{CD} 4{ }^{+} \mathrm{T}$ cells did not differ between the three strains (Figure 3.10b). This indicated that the lack of IL-4 had no effect on the expansion of Th2 cells (as marked by AmCyan expression) within the dLN. The apparent decrease in the proportion of $\mathrm{AmCyan}^{+} \mathrm{CD} 4^{+} \mathrm{T}$ cells in the IL-4 deficient mice correlated with a non-significant expansion in the total number of $\mathrm{CD} 4^{+} \mathrm{T}$ cells (Figure $3.10 \mathrm{c})$. These data indicates that IL-4 is not required for the generation and expansion of Th2 cells within the draining LN, however as the proportion of Th2 cells was decreased in the absence of IL-4, IL-4 may play a role in inhibiting the expansion of other CD4 ${ }^{+} \mathrm{T}$ cells during the $\mathrm{LN}$ response. 
a

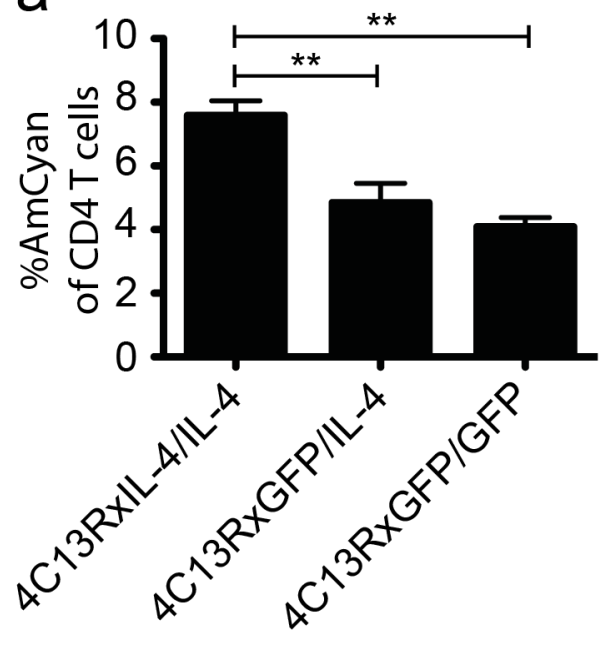

b

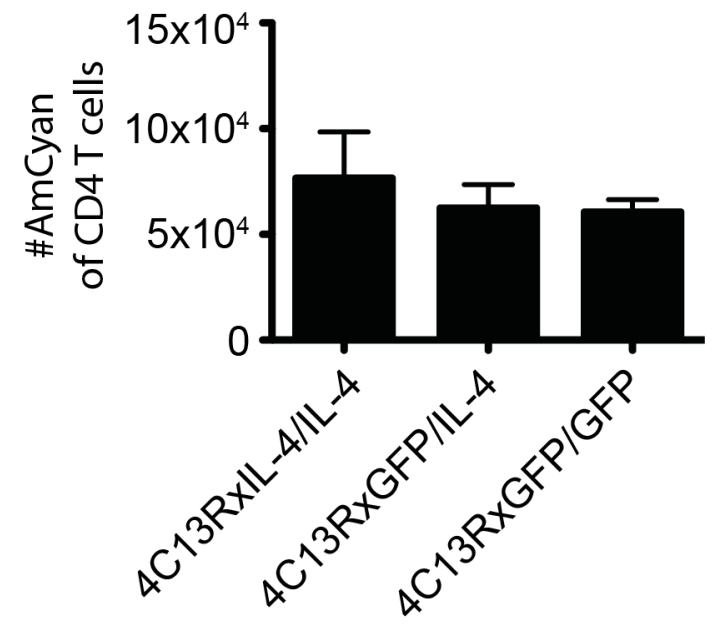

C

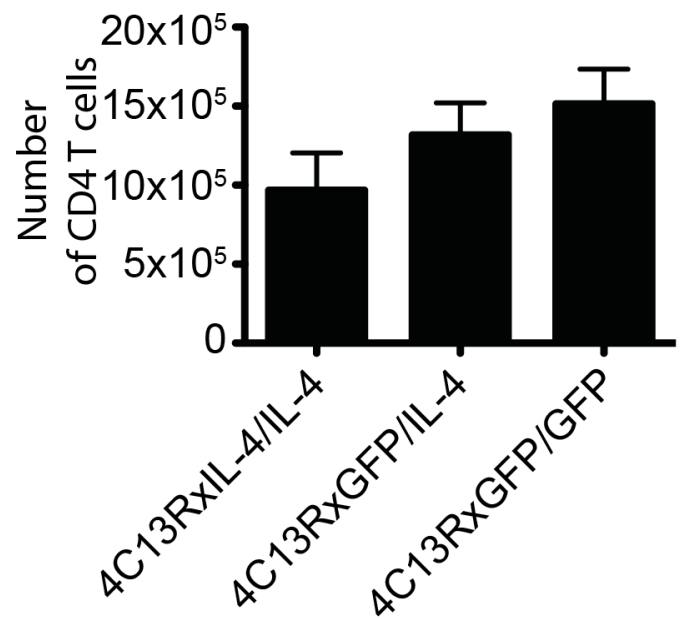

Figure 3.10 - IL-4 is not required for the expansion of IL-4 producing CD4+ $T$ cells. 4C13R reporter mice on a WT (IL-4/IL-4), heterozygous GFP/IL-4 and homozygous deficient GFP/GFP backgrounds were challenged with the pTh2 assay, $200 \mu \mathrm{g}$ of HDM was injected intradermally into the ears of each mouse and LN responses on day 7 assessed by flow cytometry. a. Percentage of AmCyan expressing CD4+ $\mathrm{T}$ cells per LN. b. Number of AmCyan expressing $\mathrm{CD}^{+} \mathrm{T}$ cells per $\mathrm{LN}$. c. Total number of $\mathrm{CD} 4^{+} \mathrm{T}$ cells per $\mathrm{LN}$ from the challenged strains. Bar graphs show mean and S.E.M. $\mathrm{n} \geq 4$ in each group. Data are representative of two independent experiments. Statistics were calculated using One-way ANOVA with Bonferroni's multiple comparisons post-test $* * \mathrm{P}<0.01$ 


\subsection{Discussion}

In this chapter I characterised a novel reporter mouse for monitoring the expression of IL-4, comparing and contrasting it with the currently available IL-4 reporter systems. I then utilised crosses of these reporter mice strains to observe, and measure what affects IL-4 expression by Th2 cells, and how IL-4 signalling controls Th2 cells differentiation and expansion in vivo. It was found that the 4C13R transgenic reporter system is an effective reporter of Th2 induction, however because AmCyan, like the parental alleles, is under allelic regulation not every cell expressing IL-4 expressed AmCyan. Despite this the reporter could be used to demonstrate that bi-allelically expressing cells were likely to be expressing a third transgenic allele and that IL-4 had little effect on the induction and expansion of Th2 cells within the reactive LN.

Allelic expression of genes, including IL-4, IL-5 and IL-13 has been widely reported ${ }^{225,226}$ but in the case of the Type 2 cytokines little is understood about what mechanisms regulate the restricted expression of these genes. Previous work focussing on the allelic nature of IL-4 demonstrated that antigen concentration correlated with increased biallelism, however these studies used heterozygous huCD2/IL-4 reporter mice, which meant the results were difficult to interpret ${ }^{288}$. The same study found the proportion of IL-4 producing CD4 ${ }^{+} \mathrm{T}$ cells correlated with antigen dose, so with greater amounts of stimulation more IL-4 was secreted into the culture. Autocrine or paracrine signalling by IL-4 on the stimulated CD4+ ${ }^{+}$cells may affect the capability of a cell to express IL-4 biallelically. By using IL-4 deficient huCD2/GFP reporter mice in Th2 conditions where the amount of IL-4 was consistent, the analysis of the role of TCR stimulation on biallelism was possible (Figure 3.5). I found that bi-allelism correlated with the amount of TCR stimulation independently of IL-4, indicating that the limiting factor that regulates allelic expression can be controlled via pathways downstream of the TCR.

Whether the allelic pattern of IL-4 expression displayed by a particular CD4 ${ }^{+} \mathrm{T}$ cell is maintained through time and through subsequent proliferation cycles has been addressed by several publications with varying interpretations. Experiments assessing the long term allelic expression of Th2 clones isolated via limiting dilution or sorting indicated that clones maintained a similar allelic pattern over many rounds of stimulation.227, 288 Conversely, sorting and restimulation of recently differentiated Th2 cells found that these cells did not maintain their sorted allelic phenotype. Upon restimulation the proportions of cells expressing cytokine using each allele were similar regardless of their sort 
phenotype $^{227}$. These experiments were undertaken in models there IL-4 is secreted, either using heterozygous reporter mice or mice with PCR identifiable single nucleotide polymorphisms in their IL-4 alleles ${ }^{305}$. The role of IL-4 on allelic maintenance is unknown. Furthermore in systems where intracellular IL-4 staining was compared to reporter to identify allelically producing cells, cells were harvested at different times to assess expression by reporter or intracellular staining ${ }^{227}$. Using the huCD2/GFP reporter mice allowed allelic maintenance to be assessed independently of IL-4 and to accurately identify mono and bi-allelic cells simultaneously post restimulation (Figure 3.7). The culture model was most similar to publications assessing recently activated Th2 cells, and consistent with these publications, I found that regardless of the phenotype at sort, all populations had very similar allelic expression after restimulation. These data are at odds with the observations using clones and may indicate that there are two phases regulating allelic expression. Together this indicates there may be an initial flexible stage during which the allelic phenotype of a cell may be changeable, but upon multiple stimulations the phenotype becomes set and inherited by the subsequent daughter cells.

The use of transgenic reporter mice was intended to allow identification of cytokine expressing Th2 cells without effecting the parental IL-4 or IL-13 alleles. The IL-4 reporting of Th2 stimulated 4C13R CD4+ $\mathrm{T}$ cells followed similar kinetics to the GFP and huCD2 reporter systems (Figure 3.2) and despite being a transgenic reporter is also under allelic regulation (Figure 3.8). This was an unexpected result; I had hypothesised that the random insertion of the transgenic construct into the genome would mean that the AmCyan allele might be missing some of the regulatory elements that mediate the expression of the parental IL-4 alleles within the Th2 locus. Two interpretations are available for the fact that the AmCyan construct is under allelic control. Either allelism is controlled by a limited availability of transcriptional machinery that are shared between the parental and transgenic alleles, or that the regulatory elements that regulate the accessibility of the IL-4 allele are also present and active within the transgene construct. The triple reporter mice highlighted an interesting phenomenon in that a cell that was expressing cytokine from two alleles was very likely to be expressing the third allele as well. This indicated that in bi-allelically expressing cells there was excess of the required factors for allelic expression, either transcription factors or mechanisms controlling transcriptional access (Figure 3.9). Unfortunately these experiments were insufficient to allow identification of the mechanisms controlling allelic expression, but they do highlight the fact that transgenic reporters are under similar control to parental IL-4 alleles. 
Therefore the 4C13R reporter cannot be used to identify every IL-4 expressing CD4 ${ }^{+} \mathrm{T}$ cell, though it remains a useful reporter for measuring changes in IL-4 expression at the population level.

The decrease in proportions but not numbers of $\mathrm{AmCyan}^{+} \mathrm{CD}^{+} \mathrm{T}$ cells in the IL-4 deficient mice (Figure 3.10) indicates that while IL-4 may not effect Th2 cell expansion it may play a role in the proliferation of other $\mathrm{CD}^{+} \mathrm{T}$ cell subsets, and in the absence of IL-4 signalling these subsets are allowed to expand. This was supported by the increase, albeit non significant, in numbers of $\mathrm{CD}^{+} \mathrm{T}$ cells in the absence of IL-4. This data supports previous work using the GFP/IL-4 and GFP/GFP reporter mice to assess the generation of IL-4 expressing Th2 cells in the absence of IL-4 during $\mathcal{N}$. brasiliensis infection, where the induction of IL-4 reporting Th2 cells occurred if there was IL-4 available or not. However my data does not marry with the previous observations between the number of $\mathrm{GFP}^{+}$cells in the GFP/IL-4 and GFP/GFP mice. With IL-4 independent Th2 expansion, it would be expected that GFP/IL-4 mice would have less $\mathrm{GFP}^{+}$cells than the GFP/GFP mice. Although the total numbers of Th2 cells should be the same in both strains, some of the cells in the GFP/IL-4 mice would be GFP- as they would be expressing mono-allelically from the WT IL-4 allele. It is not clear from these experiments why a greater number of $\mathrm{GFP}^{+}$cells than expected in the GFP/IL-4 mice has been reported with $\mathcal{N}$. brasiliensis infection. However in a model of keyhole limpet hemocyanin induced airways inflammation there was a non-significant difference between the GFP/IL-4 mice and GFP/GFP mice indicating there may be a model-biased requirement for IL- $4^{31}$. Further experimentation utilising the tools that I developed for this project will confirm the role of IL-4 in in vivo Th2 cell activation across a range of models.

\subsection{Conclusions}

In this chapter I assess the mechanisms that regulate IL-4 expression and the role of IL-4 in the expansion of Th2 cells in vivo, this was achieved by utilising various IL-4 reporter mice. The 4C13R transgenic reporter mouse is a useful tool for identifying IL-4 producing populations in an immune competent system. However, the allelic regulation of IL-4 expression, which also affects the 4C13R AmCyan reporter allele, means interpretations made using this tool must take into account some limitations. The kinetics of AmCyan expression is similar to knock-in IL-4 reporter mice, and as with the other strains not all Th2 cells that are induced to produce IL-4 will be marked by AmCyan 
expression. The fact that the proportion of bi-allelically expressing cells increases with increased TGR stimulation indicates that the mechanisms that mediate the allelic regulation of IL-4 are directly under the control of the TCR. The lack of maintenance of the allelic phenotype by restimulated $\mathrm{CD}^{+} \mathrm{T}$ cells also indicates that, at least with limited cycles of stimulation, there is plasticity in the allele that a $\mathrm{CD} 4^{+} \mathrm{T}$ cell can express from. 


\section{$4 \mathrm{IL}-13$ production by $\mathrm{CD}^{+} \mathrm{T}$ cells is regulated separately to IL-4}

\subsection{Introduction}

The functions of IL-13 have been widely studied with well-characterised roles in allergies $^{191,192}$, fibrosis ${ }^{12}$ and helminth expulsion ${ }^{198}$, and putative roles in many diseases from breast cancer ${ }^{306}$, lymphomas ${ }^{307}$ and glioma ${ }^{308}$ to myocarditis ${ }^{309}$ and regulation of glucose metabolism ${ }^{310}$. Despite many of the functions having been elucidated the mechanisms that regulate IL-13 expression by CD4 ${ }^{+} \mathrm{T}$ cells are not fully understood. In this chapter I utilise the novel IL-4 and IL-13 transgenic reporter mice to address the different signals that induce IL-4 and IL-13 expression by $\mathrm{CD} 4^{+} \mathrm{T}$ cells.

IL-4 expression has been used as an indicator for Th2 cell differentiation and thus much is known about the signals that induce IL-4 production from activated CD4+ $\mathrm{T}$ cells. Both IL-4 and IL-13 have been previously detected being produced by the same restimulated $\mathrm{CD} 4^{+} \mathrm{T}$ cells ${ }^{226}$, so it has been assumed the signals that drive IL-4 are the same that drive IL-13. In this chapter I have compared IL-4 and IL-13 expression by in vitro differentiated Th2 cells, and have found that the expression of IL-13 is under differential control to IL-4.

IL-13 expression by CD4 T cells is directly dependent upon GATA3 expression, and unlike IL-4, the ability to express IL-13 is lost if GATA3 is knocked out of already 
differentiated Th2 cells ${ }^{84}$. The level of GATA3 expression has been used to explain the phenomena whereby only $\mathrm{CD} 4{ }^{+} \mathrm{T}$ cells in the lung, but not in the $\mathrm{LN}$ of a $\mathcal{N}$. brasiliensis infected animal express IL-13 $3^{311}$. I have further investigated the absence of IL-13 expression from the murine LN using the immune competent dual cytokine reporter mice. Utilising FTY720, which targets sphingosine-1 phosphate receptors on lymphocytes and inhibits their egress from secondary lymphoid tissues ${ }^{312}$, the effects of tissue location and migration on inducing IL-13 expression by $\mathrm{CD} 4^{+} \mathrm{T}$ cells have been assessed.

\subsubsection{Aims}

These experiments assessed the expression of IL-13 by Th2 differentiated CD4 ${ }^{+} \mathrm{T}$ cells, the induction of expression both in vitro and in vivo, and the mechanisms controlling this expression in a range of models. Using the 4C13R reporter mice I assessed the factors required for activated $\mathrm{CD} 4^{+} \mathrm{T}$ cells to begin expressing IL-13 and compared these to IL-4 production. Specifically these experiments aimed to:

- Assess the timing of IL-13 expression by $\mathrm{CD} 4^{+} \mathrm{T}$ cells activated in vivo and in vitro

- Use in vitro models to measure the effects of exogenous cytokines, resident tissue of the CD4+ ${ }^{+}$cells and amount of TCR stimulation on the induction of IL-13

- Compare the expression of IL-13 by lung and LN residing CD4+ ${ }^{+}$cells both in primary and secondary immune responses

- Assess the effects of inhibiting LN egress on $\mathrm{CD}^{+} \mathrm{T}$ cell IL-13 expression in lungs and LN

\subsection{Results}

\subsubsection{Th2 differentiated $\mathrm{CD}^{+} \mathrm{T}$ cells begin expressing IL-4 and IL-13 at different times in vitro}

Consistent IL-4 expression kinetics had been observed in the previously described Th2 culture system using all three of the available IL-4 reporter models, with CD4+ ${ }^{+} \mathrm{T}$ cells dependably expressing reporter molecules on day 2 of culture (Figure 3.2) The kinetics of $\mathrm{CD}^{+} \mathrm{T}$ cell IL-13 expression was similarly assessed during the Th2 culture system using cells from the 4G13R reporter mice. When these cells were analysed for reporter 
expression daily during the culture it was noted that IL-13 reporting through DS-Red expression was not detected until day 4 of the culture (Figure 4.1a). Furthermore IL-13 reporting cells were not all simultaneously expressing IL-4; in fact the majority of IL$13^{+}$cells were reporter negative for IL-4 (Figure 4.1b) indicating that multiple Th2 populations can be identified.

Using cells from the $4 \mathrm{C} 13 \mathrm{R}$ reporter mice it was found that IL-13 expression is delayed until 2 days after IL-4 in the Th2 culture system. Unlike the traditional view of simultaneous expression of IL-4, IL-13 and IL-5 by Th2 cells, populations of cells only expressing single cytokines were detected alongside dual positive populations. This demonstrates significant difference between $\mathrm{CD} 4^{+} \mathrm{T}$ cell expression of IL-4 and IL-13. 
a

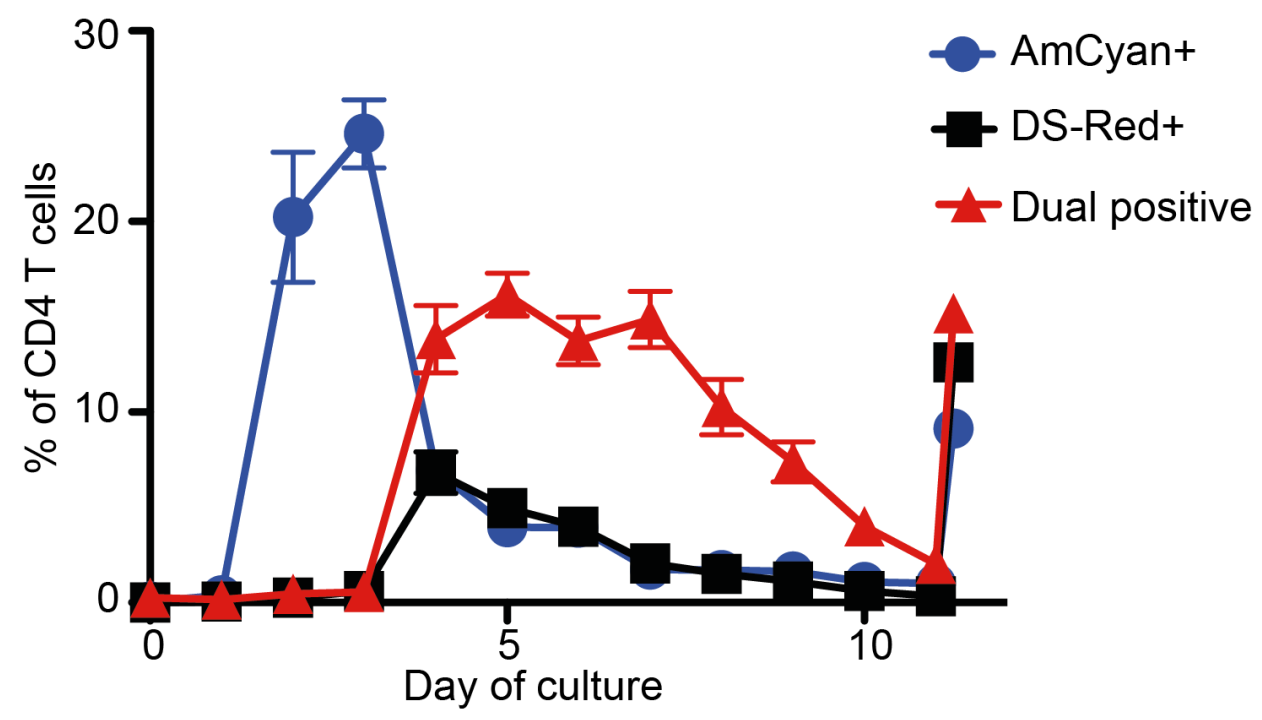

b

Day 1

Day 2

Day 4

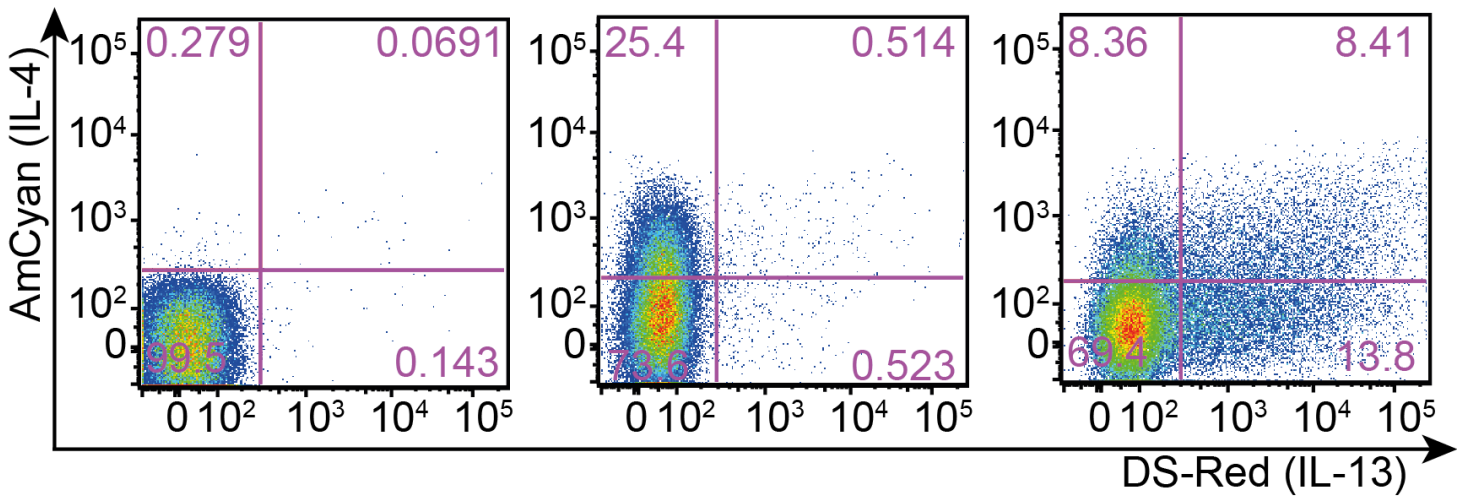

Figure 4.1 - AmCyan and DS-Red are expressed at different times in vitro.

a. Proportion of $\mathrm{CD}^{+} \mathrm{T}$ cells expressing AmCyan, DS-Red or both reporters during the course of the Th2 culture system (as described in Figure 3.1a). Plots show mean \pm S.E.M. $n=3$ in each group. Data are representative of two independent experiments. b. Representative plots showing AmCyan and DS-Red expression by $\mathrm{CD}^{+} \mathrm{T}$ cells on the indicated days, gated on Live $\mathrm{CD} 3^{+} \mathrm{CD} 4^{+}$. Plots are representative of many experiments. 
The Th2 stimulation culture protocol mandated feeding the cultures by removing half the volume and replacing it with fresh media and cytokines on day 3. I hypothesised that the supplementing of the Th2 culture system on day 3 was inducing IL-13 expression by day 4 . To check if the addition of cytokines was causing IL-13 expression, the Th2 culture system was modified, initially stimulating the cells on day 0 in Th2 conditions then changing how the cultures were fed on day 3. Feeding with IL-2 alone on day 3 slightly increased the proportion of IL-13 reporting cells, and feeding everyday with IL-2 and IL-4 decreased it, compared with the standard feeding regime of IL-2 and IL-4 on day 3 (Figure 4.2a). However not feeding with any cytokines on day 3 made no significant difference to the IL-13 reporting proportion on day 4 compared to normal conditions. This means that addition of cytokines on day 3 was not the signal that induced IL-13 reporting from the CD4+ ${ }^{+}$T cells on day 4 (Figure 4.2a).

There has been indication by some publications that IL-1 family cytokines may drive differentiation of the different T helper cell phenotypes, with IL-1 associated with Th17 function, IL-18 with Th1 and IL-33 with Th2267. Furthermore it has been shown that IL-33 induces strong IL-13 expression from ILC2 $\mathrm{s}^{253}$. In light of this I tested if IL-33 could effect IL-13 expression by CD4 ${ }^{+} \mathrm{T}$ cell. IL-33 was added to the Th2 culture system at a concentration of $10 \mu \mathrm{g} / \mathrm{mL}$, an amount able to induce responses by other cultured cell types ${ }^{313}$. It was found that IL-33 did not change the kinetics or the magnitude of IL-4 or IL-13 induction by $\mathrm{CD} 4^{+} \mathrm{T}$ cells in the Th2 differentiation culture system (Figure $4.2 \mathrm{~b})$.

Another possibility was that non-CD4 ${ }^{+}$splenocytes in the culture were signalling, either via cell-cell contact or by producing cytokines that will initiate IL-13 expression by the $\mathrm{CD}^{+} \mathrm{T}$ cells in culture. These cells could themselves be responding to the IL-4 within the culture, promoting the production of signals which would drive IL-13 expression by the $\mathrm{CD}^{+} \mathrm{T}$ cells. To test whether other cells are required for the IL-13 expression reporter expression by $\mathrm{CD} 4{ }^{+} \mathrm{T}$ cells was analysed in a total splenocyte $\mathrm{Th} 2$ culture or by $\mathrm{CD} 4{ }^{+} \mathrm{T}$ cells enriched by magnetic bead isolation prior to culture in the Th2 system. Enriched CD4 ${ }^{+} \mathrm{T}$ cells did still express both IL-4 and IL-13 with the same kinetics as those cultured with other splenocytes, however the proportion of IL-4 and IL-13 reporter positive cells was lower in the enriched cultures (Figure 4.2c). 
a
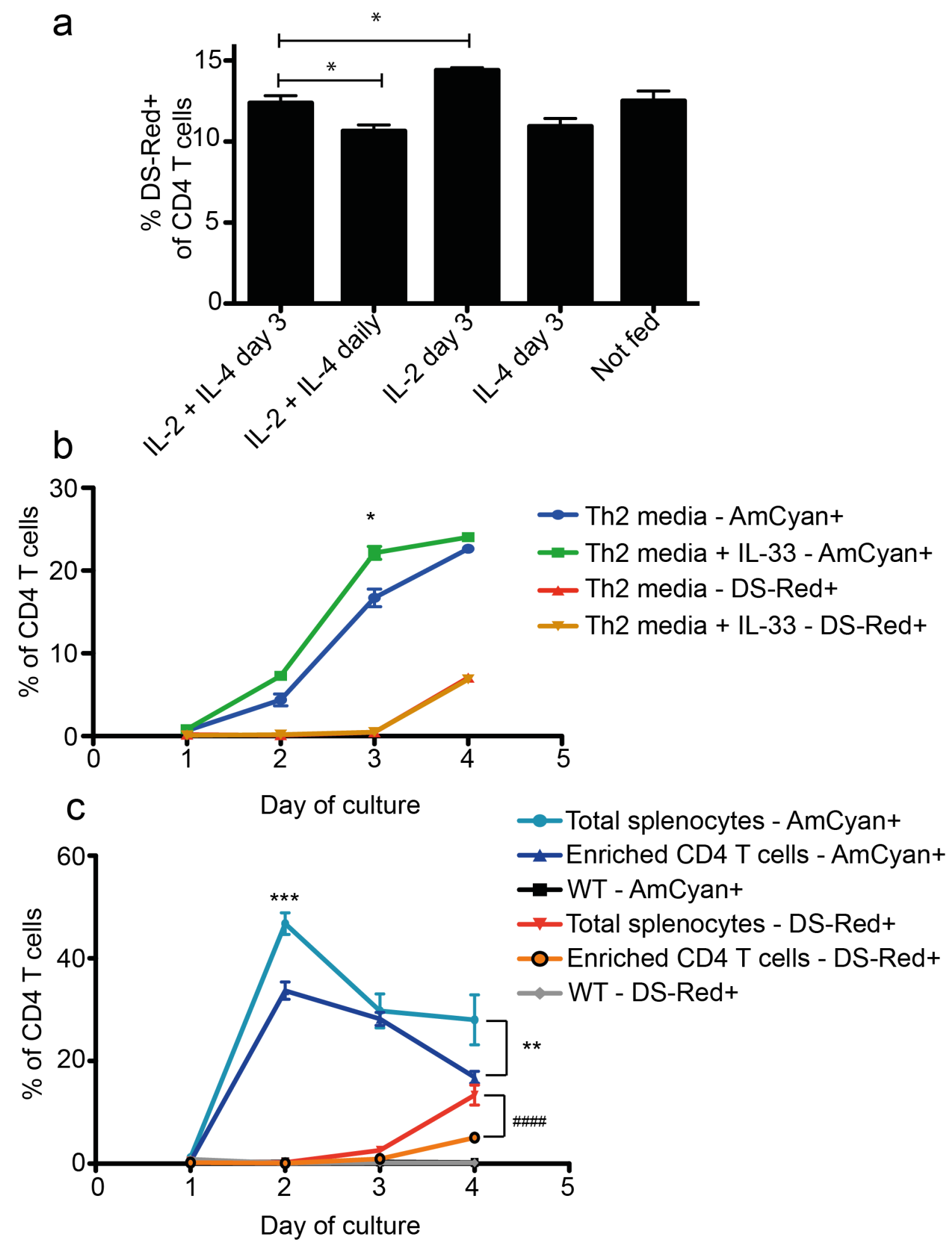

Figure 4.2 - Neither feeding with Th2 culture cytokines, adding IL-33 nor enriching for GD4 induces earlier IL-13 induction.

a. Proportion of $\mathrm{CD}^{+} \mathrm{T}$ cells expressing DS-Red at day 4 of the Th2 culture system from cultures fed on day 3 with the indicated cytokine mixes, IL-2 + IL-4 day 3 is standard Th2 culture conditions. Bar graph shows mean \pm S.E.M. $n=3$ in each group. Data are representative of two independent experiments. Statistics were calculated for each cytokine using One way ANOVA with Dunnett's multiple comparisons test $* \mathrm{P}<0.05 \mathbf{b}$. Proportion of CD $4^{+} \mathrm{T}$ cells expressing AmCyan and DS-Red cultured in the Th2 system with or without the addition of 10 $\mu \mathrm{g} / \mathrm{mL}$ IL-33. c. Proportion of CD4 ${ }^{+} \mathrm{T}$ cells expressing AmCyan and DS-Red from total 
splenocytes or enriched CD4 ${ }^{+} \mathrm{T}$ cells cultured in the Th2 system. Plots show mean \pm S.E.M. $\mathrm{n}=3$ in each group. Each graph is representative of three independent experiments. Statistics were calculated for each cytokine using two way ANOVA with a Bonferroni multiple comparisons post test. ** $\mathrm{P}<0.01 * * * * \mathrm{P}<0.0001$ comparing AmCyan + samples. \#\#\#\# $\mathrm{P}<$ 0.0001 comparing DS-Red+ samples.

IL-13 and IL-4 are differentially expressed with IL-13 consistently being expressed 2 days after IL-4 in the Th2 culture system. This delayed expression was unaffected by cells being fed, or not, with IL-2 and IL-4 on previous days of culture. IL-33, known for inducing IL-13 expression from other cell types, did not effect the induction of IL-13 by newly differentiated $\mathrm{CD}^{+} \mathrm{T}$ cells in naïve splenocyte cultures. Although other splenocytes were not required for IL-4 or IL-13 expression in the Th2 culture system their absence led to diminished proportions of reporter positive $\mathrm{CD} 4^{+} \mathrm{T}$ cells.

\subsubsection{Sustained or multiple TCR engagements are required for IL-13 expression by $\mathrm{CD4}^{+} \mathrm{T}$ cells}

In the Th2 cultures described above, splenocytes were harvested from mice that had received no experimental manipulations (naïve). As these mice are kept in only specific pathogen free, and not antigen free, conditions they have circulating populations of previously activated, memory $\mathrm{CD} 4^{+} \mathrm{T}$ cells, the majority of which have responded to antigens from commensal microbiota or diet ${ }^{314}$. Whether these previously activated cells would report IL-4 or IL-13 expression differently from true naïve CD4 ${ }^{+} \mathrm{T}$ cells had not been established. To assess this $\mathrm{CD} 4^{+} \mathrm{T}$ cells were sorted from the spleens of mice based upon their expression of CD44 and CD62L. Naïve CD4 ${ }^{+}$T cells express high levels of CD62L, allowing egress from the high endothelial vessels (HEVs) into lymphoid structures. They express low levels of CD44 until stimulated by binding of their TCR to their cognate antigen. Once activated $\mathrm{CD} 4^{+} \mathrm{T}$ cells express high levels of CD44, and levels remain high as the cells transition to memory cells ${ }^{315,316}$. Populations of CD4 ${ }^{+} \mathrm{T}$ cells were sorted from the spleens of naïve mice using the cell surface molecules CD44 1o CD62 $\mathrm{L}^{\text {hi }}$ to define naïve CD4 ${ }^{+} \mathrm{T}$ cells (Figure 4.3a). A second population was sorted on CD44hi CD62Llo; this population represented antigen-experienced cells though using CD44 and CD62L was insufficient to distinguish between activated effector cells and memory cells (Figure 4.3a). The naïve and antigen experienced $\mathrm{CD}^{+} \mathrm{T}$ cells were cultured in the Th2 culture system and their reporter expression assessed. It was found that 24 hours after plating, a small population of antigen experienced $\mathrm{CD} 4^{+} \mathrm{T}$ cells were AmCyan positive; this was earlier than AmCyan expression had been previously 
detected in the Th2 cultures with unsorted splenocytes (b). 2 days after plating the proportions of $\mathrm{CD}^{+} \mathrm{T}$ cells reporting IL-4 expression were similar between naïve and antigen experienced cells. The antigen experienced cells did not increase their IL-4 reporting after day 2 while naïve cells more than doubled the levels of reporting by day 3, and stayed high on day 4 (Figure 4.3b). IL-13 reporting was higher in the antigen experienced cells on all days. Significant IL-13 reporting was also measured from antigen experienced cells from day 1 of culture, earlier that the day 4 when it was previously detected from unsorted splenocytes and the naïve cells (Figure 4.3b). One caveat in these experiments was that lower levels of IL-4 and IL-13 reporting were measured that were seen in experiments with unsorted splenocytes. This may be due to the lack of other splenocytes as in Figure 4.2c or because the cells are not as healthy after being sorted.

From these data, cells that had been previously activated in vivo were able to express Th2 cytokines faster that naïe cells when stimulated in vitro. Although antigen experienced cells presented higher proportions of IL-13 reporters, naïve cells had higher proportions of IL-4 reporter positive cells at the later time points. 
a Pre-sort

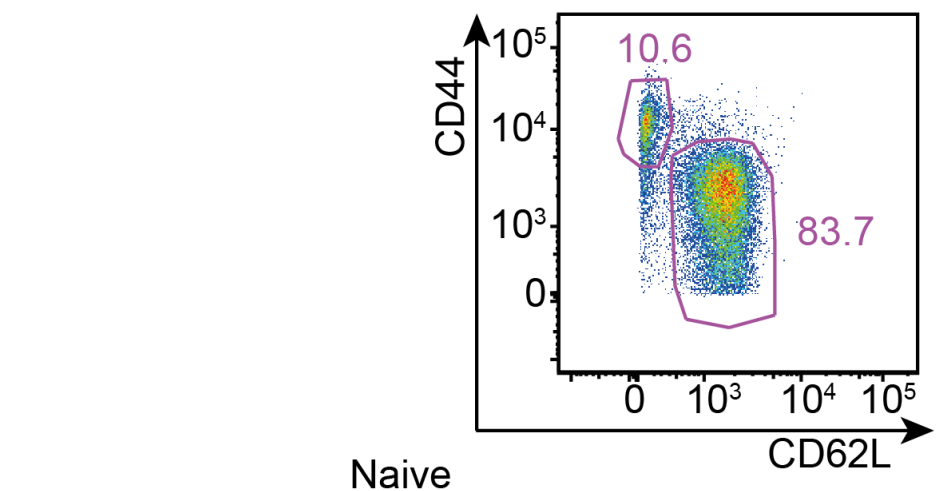

Antigen Experienced

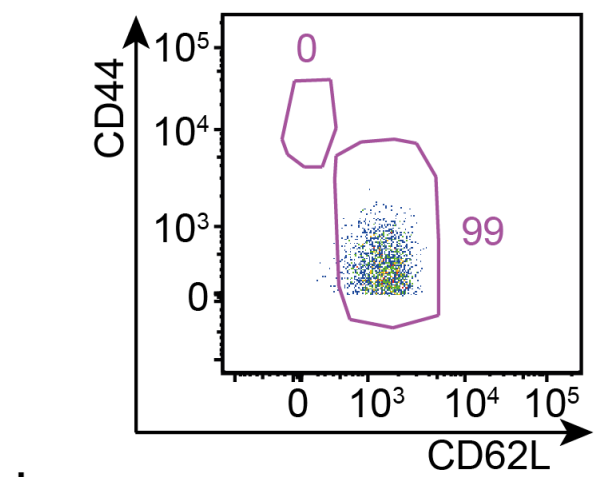

b

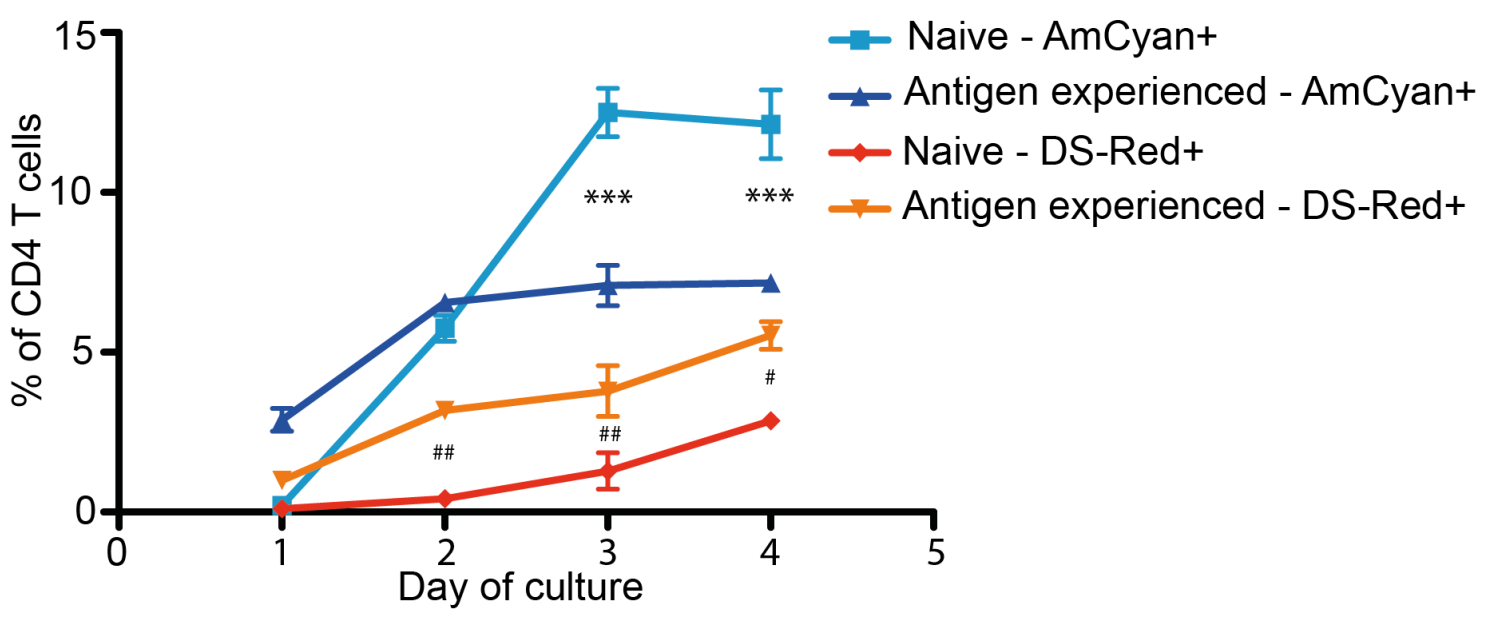

Figure 4.3 - Previously activated/memory cells express DS-Red earlier in vitro than naïve cells.

a. Representative plots of $\mathrm{CD}^{+} \mathrm{T}$ cells from splenocytes pre-sort and the two post-sort populations defined naïve and antigen experienced based upon expression of CD62L and CD44. b. Proportion of CD4 ${ }^{+}$T cells expressing AmCyan and DS-Red from the two post-sort populations during the Th2 culture system. Plots show mean \pm S.E.M n=3 in each group. Each graph is representative of two independent experiments. Statistics were calculated for each cytokine using two way ANOVA with a Bonferroni multiple comparisons post test. ${ }^{*} \mathrm{P}<0.05$ ** $\mathrm{P}<0.01 * * * \mathrm{P}<0.001$ comparing AmCyan+ samples. \# $\mathrm{P}<0.05$ \#\# $\mathrm{P}<0.01$ comparing DS-Red+ samples. 
The longitudinal effects of TCR stimulation on IL-4 and IL-13 expression by CD4 ${ }^{+} \mathrm{T}$ cells was assessed by culturing cells for varying lengths of time on $\alpha \mathrm{CD} 3$ in Th2 conditions. Each day after the initiation of the culture, some cells were removed from $\alpha \mathrm{CD} 3$ and transferred to wells with Th2 conditions but no $\alpha \mathrm{CD} 3$. The reporter expression on day 4 of culture by cells removed from TCR stimulus was compared to cells cultured for the full four days on $\alpha \mathrm{CD} 3$. The proportion of cells that were reporting IL-4 increased with the number of days the cells were exposed to $\alpha \mathrm{CD} 3$ before removal (Figure 4.4a). Cells cultured on $\alpha \mathrm{CD} 3$ for the complete four days had a significantly greater proportion expressing IL-13 reporter compared with cells removed from stimulation at any point prior to day 4 (Figure $4.4 \mathrm{~b}$ ).

It was found that in vitro Th2 differentiated CD4 ${ }^{+} \mathrm{T}$ cells will express IL-4 after only a brief stimulation in Th2 conditions, even if TCR stimulation is removed though longer stimulation does lead to increased proportions of IL-4 reporting cells. IL-13 however required continuous stimulation for its optimal expression, with rested cells only expressing IL-13 reporter slightly above background levels on day 4. These data further demonstrate the significant differences in the mechanisms controlling the expression of these two cytokines. 

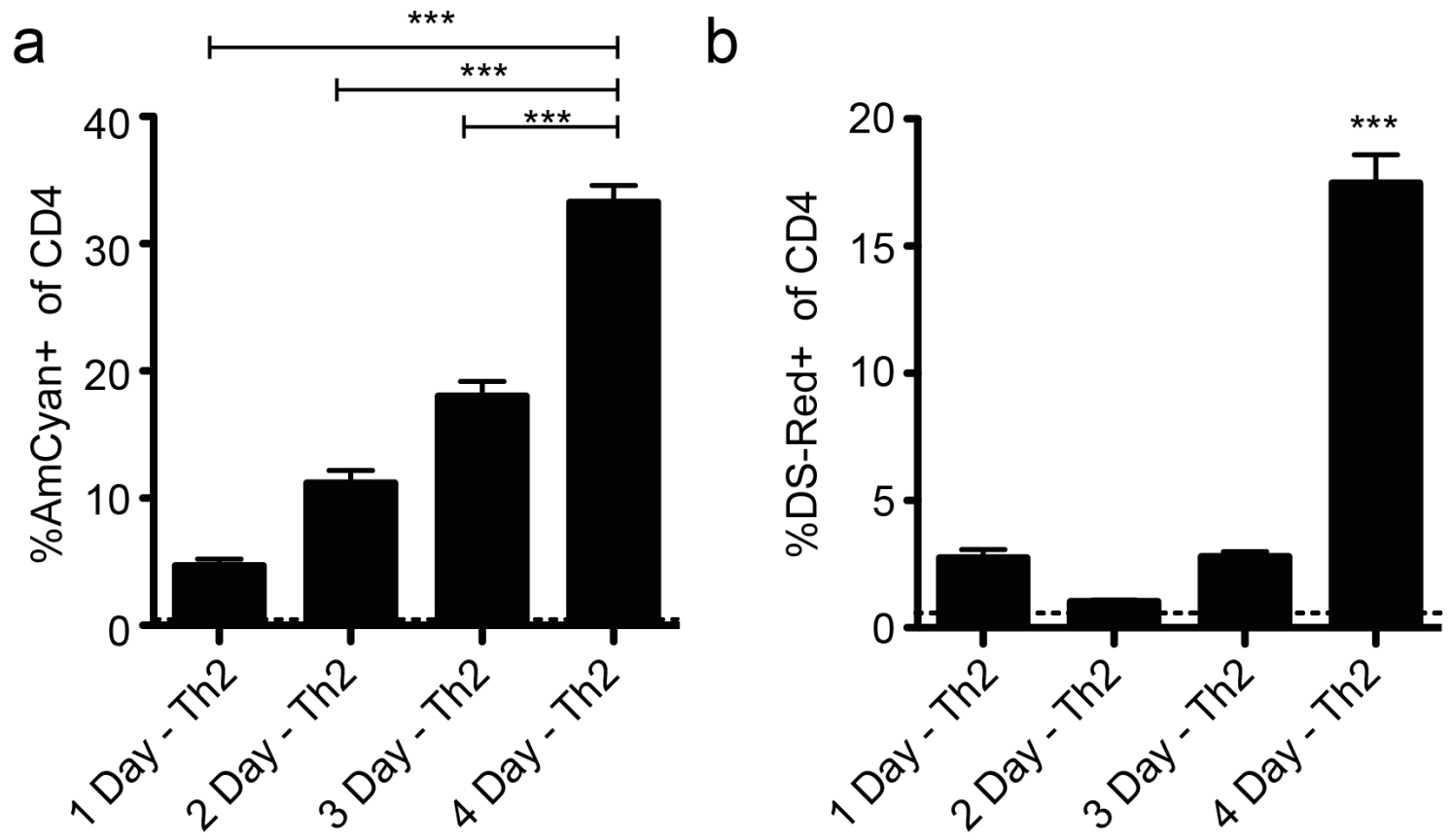

Figure 4.4 - Sustained TGR stimulation is required for IL-13 expression.

Proportion of $\mathrm{CD}^{+} \mathrm{T}$ cells expressing a. AmCyan and b. DS-Red on day 4 of the Th2 culture system. Cells were cultures in Th2 conditions in aCD3 coated plates for the indicated number of days, then moved to uncoated plates and rested in Th2 media (IL-2 and IL-4). Dashed lines indicate background reporter based upon cultured cells from non-transgenic littermates. Plots show mean \pm S.E.M. $n=3$ in each group. Data are representative of three independent experiments. Statistics were calculated for each group using One way ANOVA with Tukey's post test *** $\mathrm{P}<0.001$ 
Whilst a consistent pattern of reporter expression was observed by $\alpha \mathrm{CD} 3$ stimulated $\mathrm{CD}^{+} \mathrm{T}$ cells in the Th2 culture system, it remained unknown if this pattern would be replicated by $\mathrm{CD}^{+} \mathrm{T}$ cell stimulated in a more physiological way by antigen loaded APGs. An antigen specific system was designed where 4C13R reporter mice were crossed with TGR transgenic OT-II mice. OT-II mice have been transgenically modified so that approximately $80 \%$ of their CD4 T cells expressing the TCR specific for the OVA peptide ISQAVHAAHAEINEAGR (ISQ ${ }^{291} \cdot \mathrm{CD}^{+}{ }^{+} \mathrm{T}$ cells from 4C13RxOT-II spleens were mixed with digested splenocytes that had been pulsed with a range of ISQ concentrations. The APGs from the digested spleens endocytosed the ISQ peptides and loaded them on their MHC II molecules ${ }^{317}$ allowing presentation to, and stimulation of the 4C13RxOT-II CD4 ${ }^{+} \mathrm{T}$ cells. It was found that 4C13RxOT-II $\mathrm{CD}^{+} \mathrm{T}$ cells expressed IL-4 reporter when stimulated with ISQ pulsed APGs (Figure 4.5a), and similar kinetics were observed as had been seen with the $\alpha \mathrm{CD} 3 \mathrm{Th} 2$ culture systems (Figure 4.1) with no IL-4 reporter being detected prior to day 2 of culture. There was a dose response with the proportion of cells stimulated to report IL-4 similar with $1 \mu \mathrm{M}$ and $10 \mu \mathrm{M}$ pulsed APCs. Slightly higher levels were maintained on day 4 by the $1 \mu \mathrm{M}$ pulsed cells. CD4+ ${ }^{+}$cells cultured with $0.1 \mu \mathrm{M}$ ISQ pulsed APCs expressed much lower proportions of IL-4 reporter, while none was detected from cells cultured with the $0.01 \mu \mathrm{M}$ ISQ pulsed and unpulsed APGs (Figure 4.5a). No IL-13 reporting by $\mathrm{CD}^{+} \mathrm{T}$ cells cultures with APGs pulsed with any concentration of ISQ was detected at any time point, even day 4 when IL-13 reporting was previously observed with the Th2 culture system (Figure 4.5b). 
a

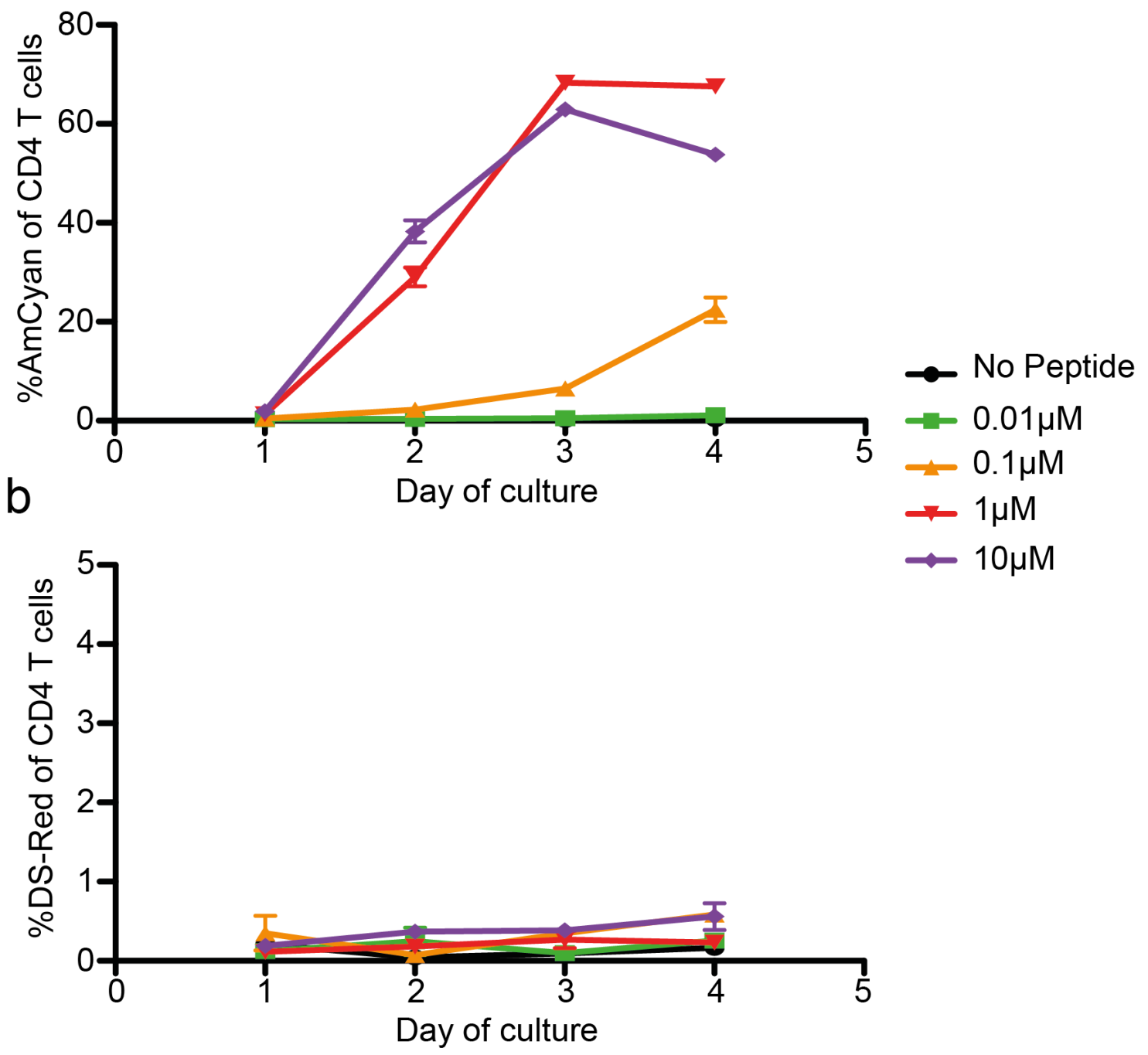

Figure 4.5 - APG presentation does not induce IL-13 expression by $\mathrm{CD4}^{+} \mathrm{T}$ cell.

Proportion of antigen specific 4C13RxOT-II CD4 ${ }^{+} \mathrm{T}$ cells expressing $\mathbf{a}$. AmCyan and $\mathbf{b}$. DSRed, when stimulated on day 0 with digested splenocytes pulsed with various concentrations of ISQ peptide. Plots show mean \pm S.E.M. $n=3$ in each group. Plots are representative of two independent experiments. 
Splenic DCs only have an approximate 3 day lifespan in vivo ${ }^{318}$ and in culture greater than $80 \%$ of the DCs die within 48 hours $^{319}$, removing antigen presentation and TCR stimulation for the $\mathrm{CD} 4^{+} \mathrm{T}$ cells. To test if the lack of continued stimulation was the reason for the lack of IL-13 expression, as seen previously in Figure 4.4, extra APCs were added on day 3 of the culture and then IL-13 expression assessed on day 4. Cultures were plated on day 0 with 4C13RxOT-II CD4 ${ }^{+} \mathrm{T}$ cells and ISQ pulsed or unpulsed APGs. On day 3 added to some wells were ISQ pulsed CD11c ${ }^{+}$cells sorted via magnetic beads from digested spleens with or without antibodies that inhibit MHC II binding to $\mathrm{CD}^{+} \mathrm{T}$ cell TCR. The addition of ISQ pulsed APGs further increased the proportion of reporter positive $\mathrm{CD}^{+} \mathrm{T}$ cells in a MHC II dependent manner, as blocking antibody inhibited this increase (Figure 4.6a). Addition of ISQ pulsed APGs on day 3 was sufficient to induce significant IL-13 reporting by the $4 \mathrm{C} 13 \mathrm{RxOT}-\mathrm{II}$ CD $4{ }^{+} \mathrm{T}$ cells 24 hours after their addition to the culture (Figure 4.6b). This IL-13 induction was also dependent on MHC II interactions as the blocking antibody diminished the proportion of IL-13 reporter positive cells by approximately $84 \%$.

From these experiments it was discovered that IL-13 is more likely to be expressed, and expressed sooner by cells that have been previously activated. In in vitro models IL-13 was not expressed by cells that had been removed from $\alpha \mathrm{CD} 3$ antibody TCR stimulation on any day before day 4. Furthermore APG stimulated CD4 ${ }^{+} \mathrm{T}$ cells did not express IL-13 either. IL-13 production could be induced from APG stimulated cells with the addition of extra peptide loaded APGs on day 3 of culture, and the expression was dependent upon MHC II interactions. 
a
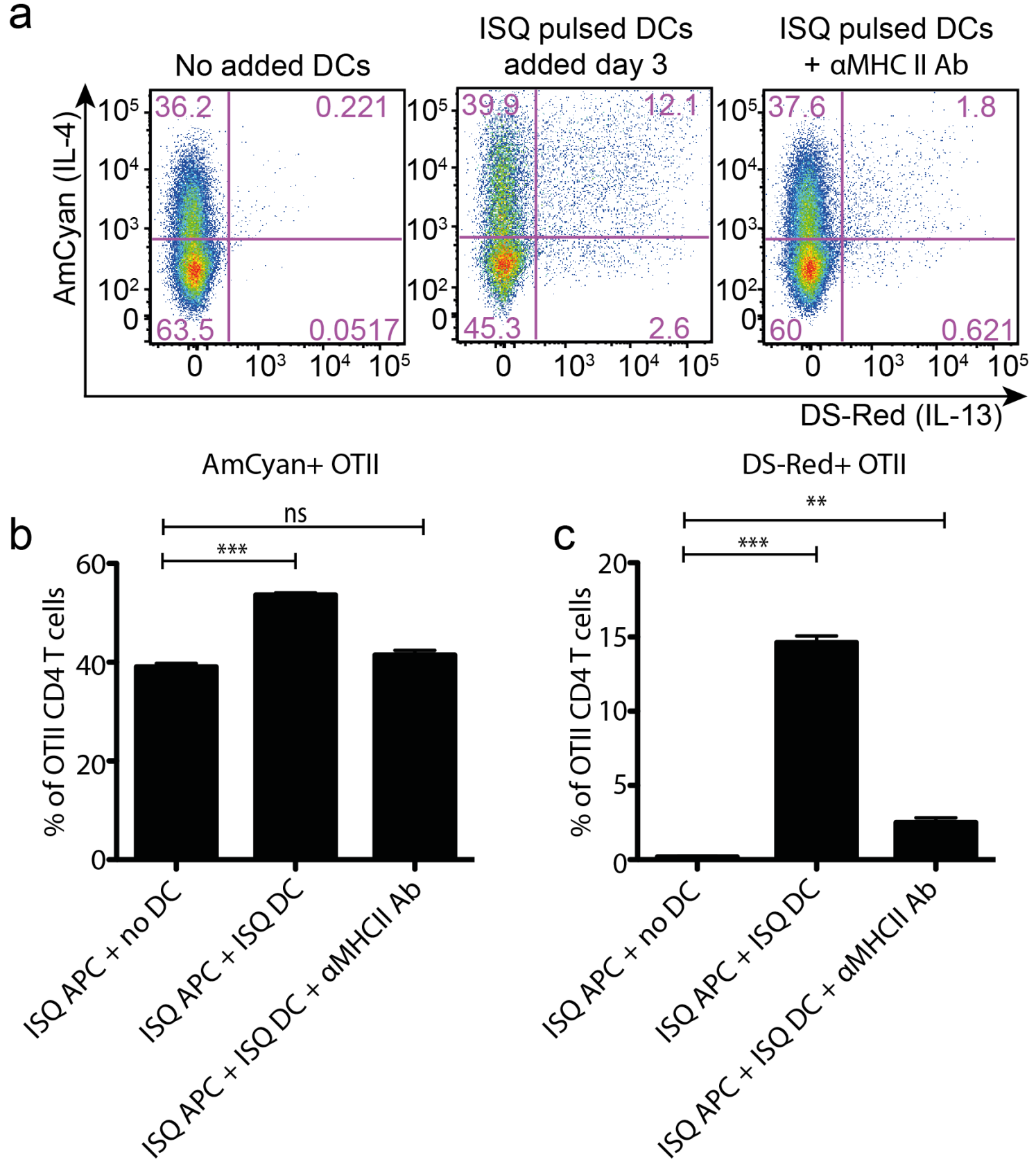

Figure 4.6 - Addition of extra peptide loaded APGs induces GD4 $^{+}$T cell IL-13 expression in a TCR dependent manner.

a. Representative flow plots and proportion of antigen specific 4C13RxOT-II CD4 ${ }^{+} \mathrm{T}^{-}$cells expressing b. AmCyan and c. DS-Red on day 4. Cells were stimulated on day 0 with digested splenocytes pulsed with ISQ peptide, ISQ pulsed DCs added day 3 of culture with or without aMHC-II antibodies. Plots show mean \pm S.E.M. $n=3$ in each group. Plots are representative of two independent experiments. Statistics were calculated for each group using One way ANOVA with Tukey's post test $* * \mathrm{P}<0.01$ *** $\mathrm{P}<0.001 \mathrm{~ns}=$ not significant. 


\subsubsection{Tissue specific $\mathrm{CD}^{+} \mathrm{T}$ cell cytokine expression patterns are not observed with in vitro stimulation}

To assess the expression of IL-4 and IL-13 reporters in vivo, 4C13R dual reporter mice were infected with $550 \mathcal{N}$. brasiliensis subcutaneously and the reporter expression by $\mathrm{CD}^{+} \mathrm{T}$ cells assessed in the mediastinal $\mathrm{LN}$ and lung at various time points post infection. Previous studies that compared IL-4 and IL-13 expression by LN and lung $\mathrm{CD}^{+} \mathrm{T}$ cells during $\mathcal{N}$. brasiliensis infection used knock-in reporter mice which have deficiencies in their abilities to produce IL-4 and IL-13 which may have unintended consequences on $\mathrm{Th} 2$ responses ${ }^{311}$. 4G13R transgenic reporter mice are capable of mounting a normal immune response as their parental IL-4 and IL-13 genes are untouched, it was unknown if the same pattern of reporter expression would be detected using this system as has been previously reported.

7 days post subcutaneous infection $4 \mathrm{C} 13 \mathrm{R}$ reporting $\mathrm{CD} 4^{+} \mathrm{T}$ cells in the lung expressed both IL-4 and IL-13 reporters (Figure 4.7a). Interestingly, reporter positive cells were detected at 2 days post infection and at this time point the parasites have only been in the lung for a maximum of 36 hours. These cells were notably IL-4 and not IL-13 positive. By day 7 IL-13 reporting was detectable but still a lower proportion of CD4 ${ }^{+} \mathrm{T}$ cells expressed IL-13 than the IL-4 reporter, while the proportions of IL-4 and IL-13 reporting $\mathrm{CD} 4^{+} \mathrm{T}$ cells were fairly equal at day 9 post primary infection (Figure $4.7 \mathrm{~b}$ ). 
a
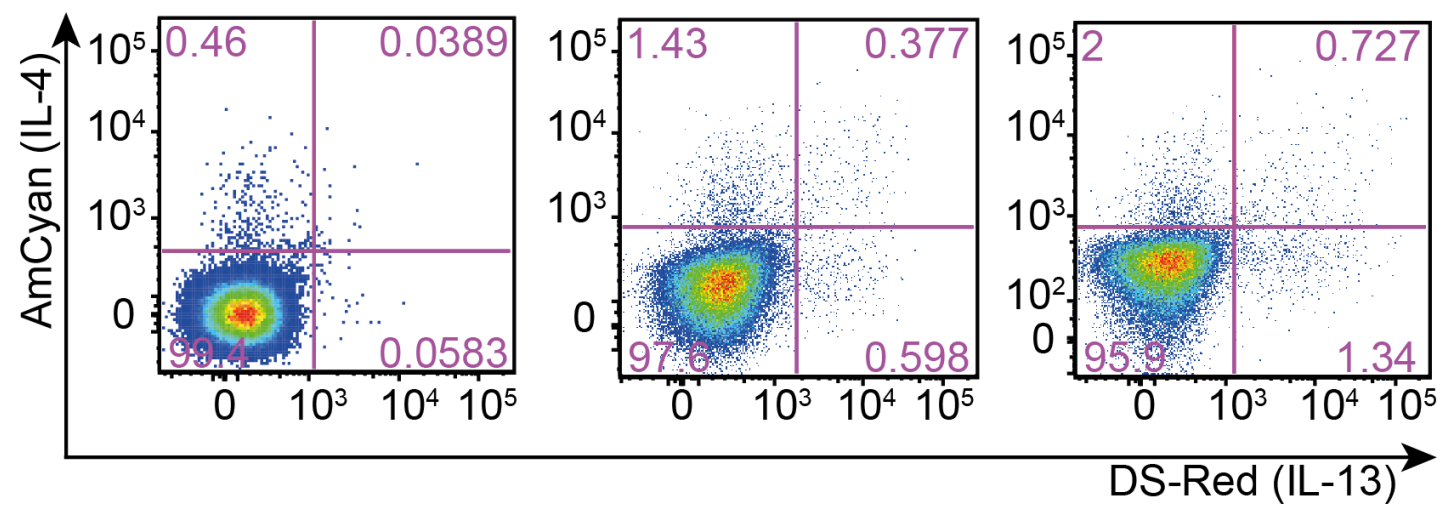

b

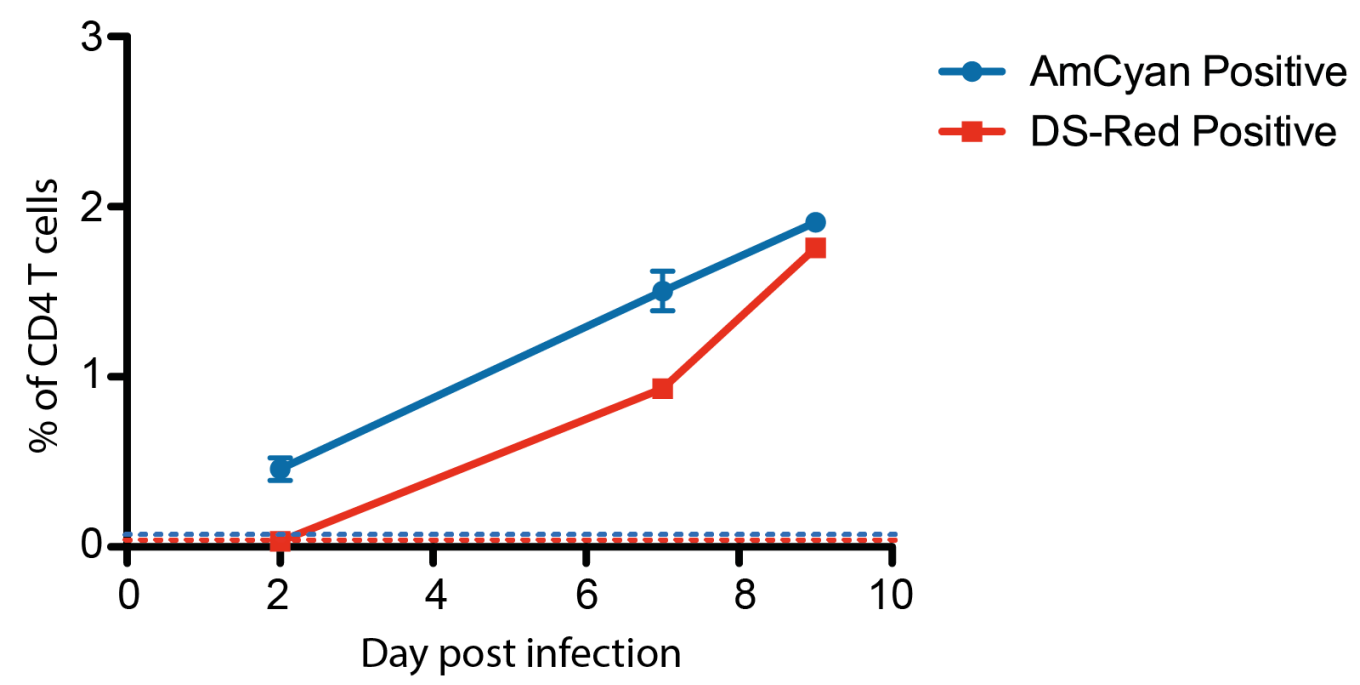

Figure 4.7 - Lung GD4 ${ }^{+} \mathrm{T}$ cells express both AmGyan and DS-Red in response to $N$. brasiliensis infection.

a. Representative plots and b. proportion of lung CD4+ T cells expressing AmCyan and DSRed at the indicated days post primary subcutaneous infection with 550 L3 $\mathcal{N}$. brasiliensis. Dashed lines indicate background based upon non-transgenic CD4 ${ }^{+} \mathrm{T}$ cells. Plots show mean \pm S.E.M. $\mathrm{n} \geq 2$ in each group. Plots are representative of two independent experiments. 
The 4C13R reporter mice were used to determine if previous reports of $\mathrm{LN} \mathrm{CD} 4^{+} \mathrm{T}$ cells limiting their expression of IL-13 could be replicated ${ }^{311}$. As had been seen by the lung $\mathrm{CD}^{+} \mathrm{T}$ cells there were robust IL-4 reporter expression by $\mathrm{CD} 4^{+} \mathrm{T}$ cells in the mediastinal LN, though the expression at day 2 was marginal and not significantly more than background. There was no significant IL-13 reporter expression detected at any time point (Figure 4.8a). To assess if this was a phenomenon specific to $\mathrm{CD}^{+} \mathrm{T}$ cells resident in the mediastinal $\mathrm{LN}$ or to all $\mathrm{LN} \mathrm{CD} 4^{+} \mathrm{T}$ cells, 4C13R mice were subjected to the pTh2 assay whereby mice were challenged with an intradermal injection of HDM and reporter expression in the auricular draining LN assessed on days 3 and 7. Repeating the pattern of reporter expression displayed by $\mathcal{N}$. brasiliensis responding mediastinal $\mathrm{LN} \mathrm{CD}^{+} \mathrm{T}$ cells, HDM responding $\mathrm{CD} 4^{+} \mathrm{T}$ cells in the $\mathrm{pTh} 2$ assay did not express detectable IL-13 reporter, while a large proportion reported IL-4 (Figure 4.8b). The 4C13R reporter mice replicated the previously demonstrated lack of IL-13 expression by activated LN Th2 cells, while lung tissue $\mathrm{CD}^{+}{ }^{+} \mathrm{T}$ cells were able to produce both IL-4 and IL-13, IL-13 is expressed later than IL-4 by these cells but eventually equal proportions of cells expressed each of the cytokines by day 9 post infection. 
a
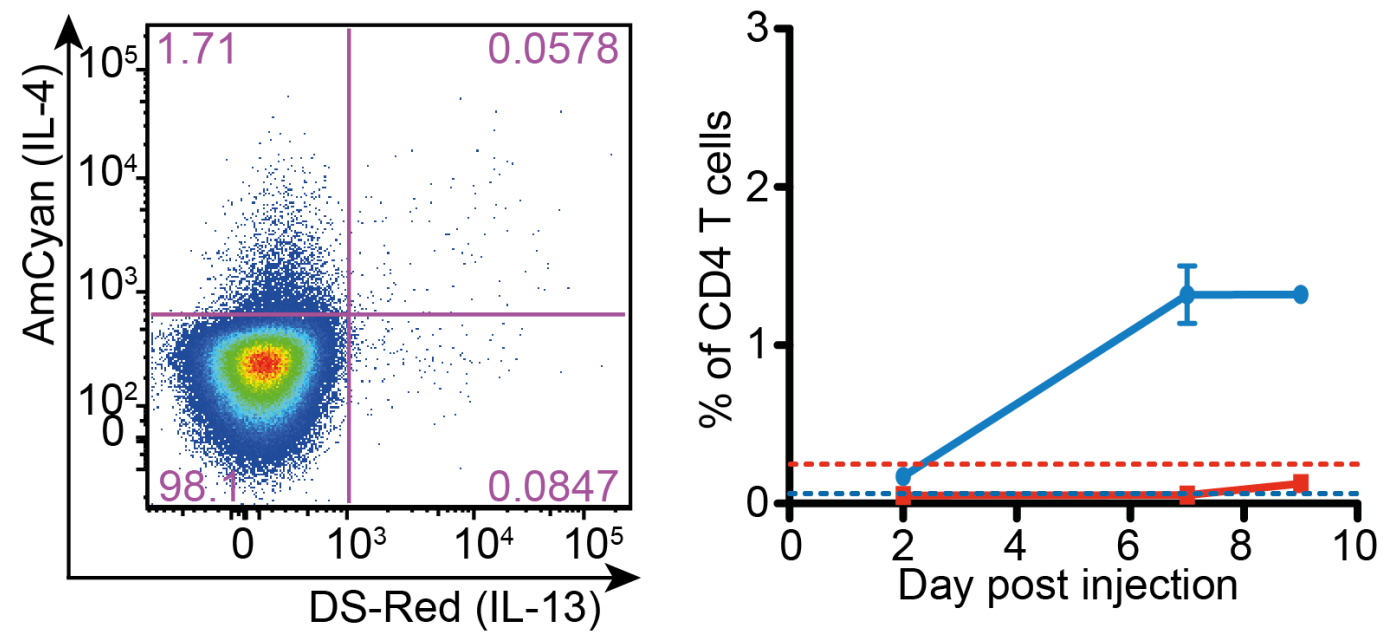

b
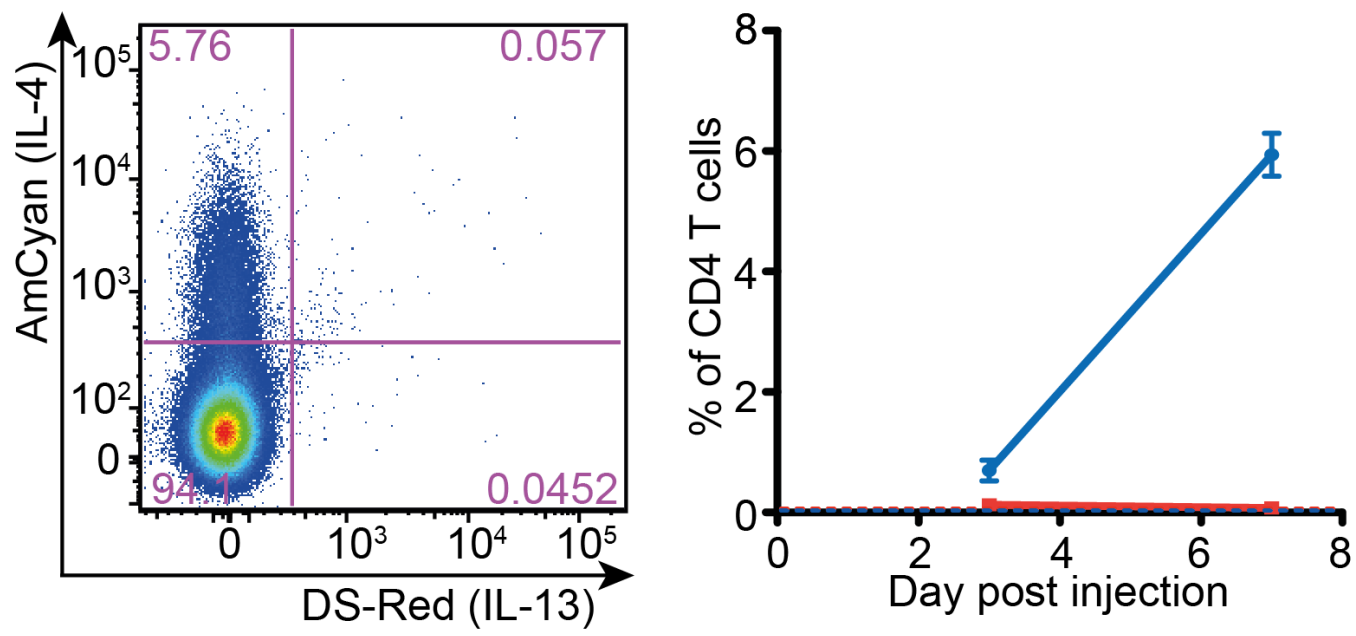

Figure 4.8 - LN CD4+ $T$ cells do not express DS-Red.

a. Plot of day 7 and proportions of $\mathrm{CD}^{+} \mathrm{T}$ cells expressing AmCyan and DS-Red in the mediastinal LN 2, 7 and 9 days after primary subcutaneous infection with 550 L3 $\mathcal{N}$. brasiliensis. Dashed lines indicate background based upon non-transgenic CD4+ T cells. Plot shows mean \pm S.E.M. $\mathrm{n} \geq 2$ in each group. Plot is representative of two independent experiments. b. Plot of day 7 and proportions of $\mathrm{CD}^{+} \mathrm{T}$ cells expressing AmCyan and DS-Red in the auricular draining LN 4 and 7 days after intradermal injection in the ear of house dust mite. Dashed lines indicate background based upon non-transgenic $\mathrm{CD} 4^{+} \mathrm{T}$ cells. Plot shows mean \pm S.E.M. $\mathrm{n} \geq 4$ in each group. Plots are representative of two independent experiments. 
Taking these results into consideration I hypothesised that $\mathrm{CD} 4^{+} \mathrm{T}$ cells resident in the lung may be phenotypically distinct from the $\mathrm{LN} \mathrm{CD} 4^{+} \mathrm{T}$ cells, and that they may be primed towards expedited IL-13 production. To assess this $\mathrm{CD} 4^{+} \mathrm{T}$ cells were isolated from the lung and their reporter expression compared to $\mathrm{CD} 4^{+} \mathrm{T}$ cells from the spleen in the Th2 culture system. CD4 ${ }^{+} \mathrm{T}$ cell IL-4 and IL-13 reporting followed similar patterns regardless if the cells were from the lung or spleen, with IL-4 detected on day 2 and IL-13 on day 4 (Figure 4.9a). The only difference was a slightly lower percentage of IL-4 reporting CD4+ $\mathrm{T}$ cells from the lung than spleen on days 2 and 3 of culture. Hence lung $\mathrm{CD}^{+} \mathrm{T}$ cell were not any more likely to express IL-13 than cells from lymphoid tissues.

$\mathrm{CD}^{+}$thymocytes were also compared to $\mathrm{CD} 4^{+} \mathrm{T}$ cells from the spleen in the $\mathrm{Th} 2$ culture system. Using CD4 expression to isolate cells from the thymus will extract not only newly matured $\mathrm{CD} 4^{+} \mathrm{T}$ cells but also double positive thymocytes in the process of becoming mature $\mathrm{T}$ cells, although many of the double positive cells will rapidly die in culture (Figure 4.9b). In a Th2 differentiating culture CD4+CD8- thymocytes express both IL-4 and IL-13; IL-4 possessed similar kinetics as spleen CD4+ $\mathrm{T}$ cells, though at a lower proportion until day 4. Interestingly low levels of IL-13 expression could be detected on day 2, 2 days before spleen derived $\mathrm{CD}^{+} \mathrm{T}$ cells become IL-13 reporter positive (Figure 4.9b). Therefore thymocytes can produce Th2 associated cytokines in Th2 culture, and the early IL-13 reporter expression may indicate a difference in activation potential between thymocytes and peripheral $\mathrm{T}$ cells. 


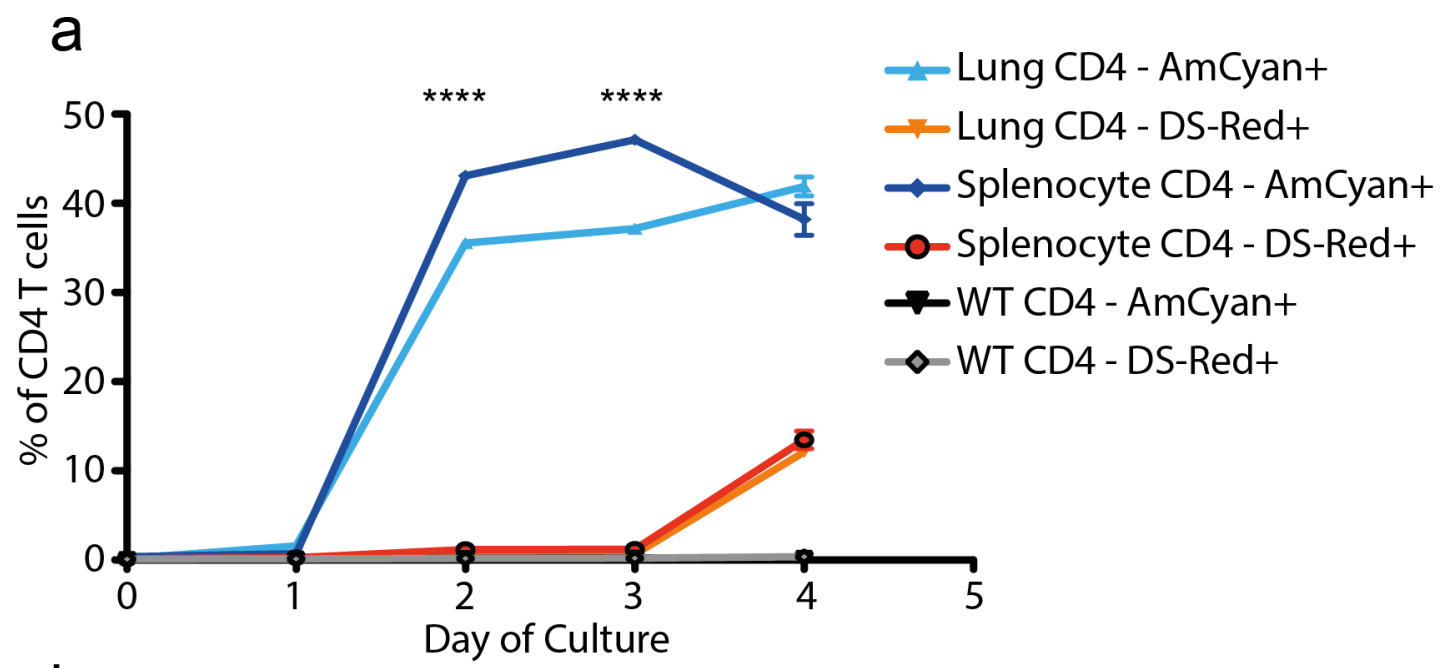

b

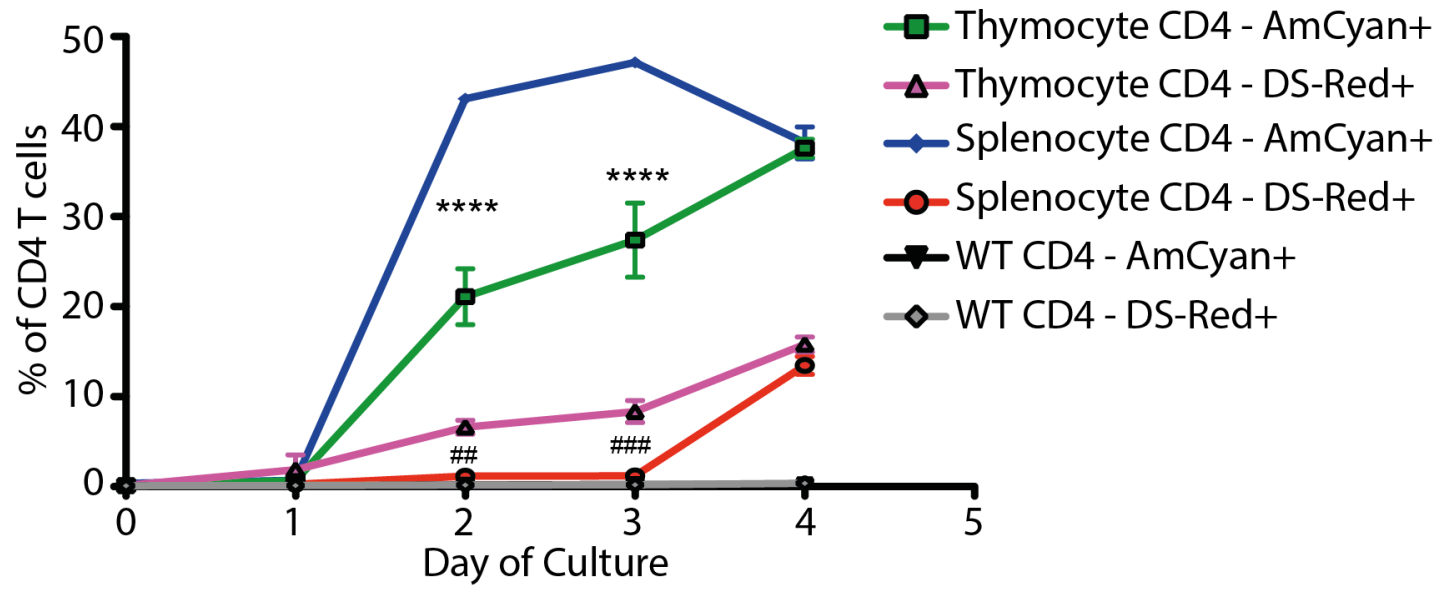

Figure 4.9 - In vitro $T h 2$ differentiated $\mathrm{CD4}^{+} \mathrm{T}$ cells from $\mathrm{LN}$ and lung display the same pattern of cytokine reporting.

a. Proportion of $\mathrm{CD} 4^{+} \mathrm{T}$ cells enriched from spleen and lungs that express AmCyan and DSRed during the Th2 culture system. b. Proportion of CD4 ${ }^{+} \mathrm{T}$ cells enriched from spleen and thymus that express AmCyan and DS-Red during the Th2 culture system. Plots show mean \pm S.E.M. $n=3$ in each group. Each graph is representative of two independent experiments. Statistics were calculated for each cytokine using two way ANOVA with a Bonferroni multiple comparisons post test. $* * * * \mathrm{P}<0.0001$ comparing AmCyan+ samples. \#\# $\mathrm{P}<0.01$ \#\#\# $\mathrm{P}<$ 0.001 comparing DS-Red+ samples. 
Considering that in vitro $\mathrm{CD} 4^{+} \mathrm{T}$ cells needed multiple interactions with $\mathrm{APC}$ s to induce IL-13 expression, it was thought that a secondary infection with a pathogen could potentially induce stronger IL-13 responses from $\mathrm{LN} \mathrm{CD}^{+} \mathrm{T}$ cells than seen in primary infection. 4G13R reporter mice were infected with $550 \mathcal{N}$. brasiliensis, allowing the infection to progress naturally, with the mice clearing the infection by day 14 post infection ${ }^{124}$. Mice were left until day 30 post primary infection to allow their immune responses to contract, and then reinfected subcutaneously with another dose of 550 larvae. The secondary $\mathrm{CD} 4{ }^{+} \mathrm{T}$ cell responses in the lung and the mediastinal $\mathrm{LN}$ were analysed. Of the reporting cells, both in the lung and the few detected in the LN, there were populations that expressed one cytokine or both IL-4 and IL-13, with the dual expressing populations less frequent than either of the single producing populations (Figure 4.10a). Total proportions of lung $\mathrm{CD}^{+} \mathrm{T}$ cells expressing IL-4 or IL-13 reporters were similar throughout the time points assessed, while there was still more IL-4 than IL-13 reporting CD4+ $\mathrm{T}$ cells in the mediastinal LN (Figure 4.10b). Again robust reporter positive cells were detected in the lung at 2 days post infection, but LN responses were also detectable at this time point of the secondary infection. Comparing secondary to primary at day 6 of infection, the peak of secondary responses, there was approximately a 13-fold increase in the number of IL-13 reporting cells in the lung, and approximately 30 -fold increase in the LN. Therefore despite more IL-13 producing cells being detected, they are still far less frequent than IL-4 reporting CD4 ${ }^{+} \mathrm{T}$ cells in the secondary LN. 
a
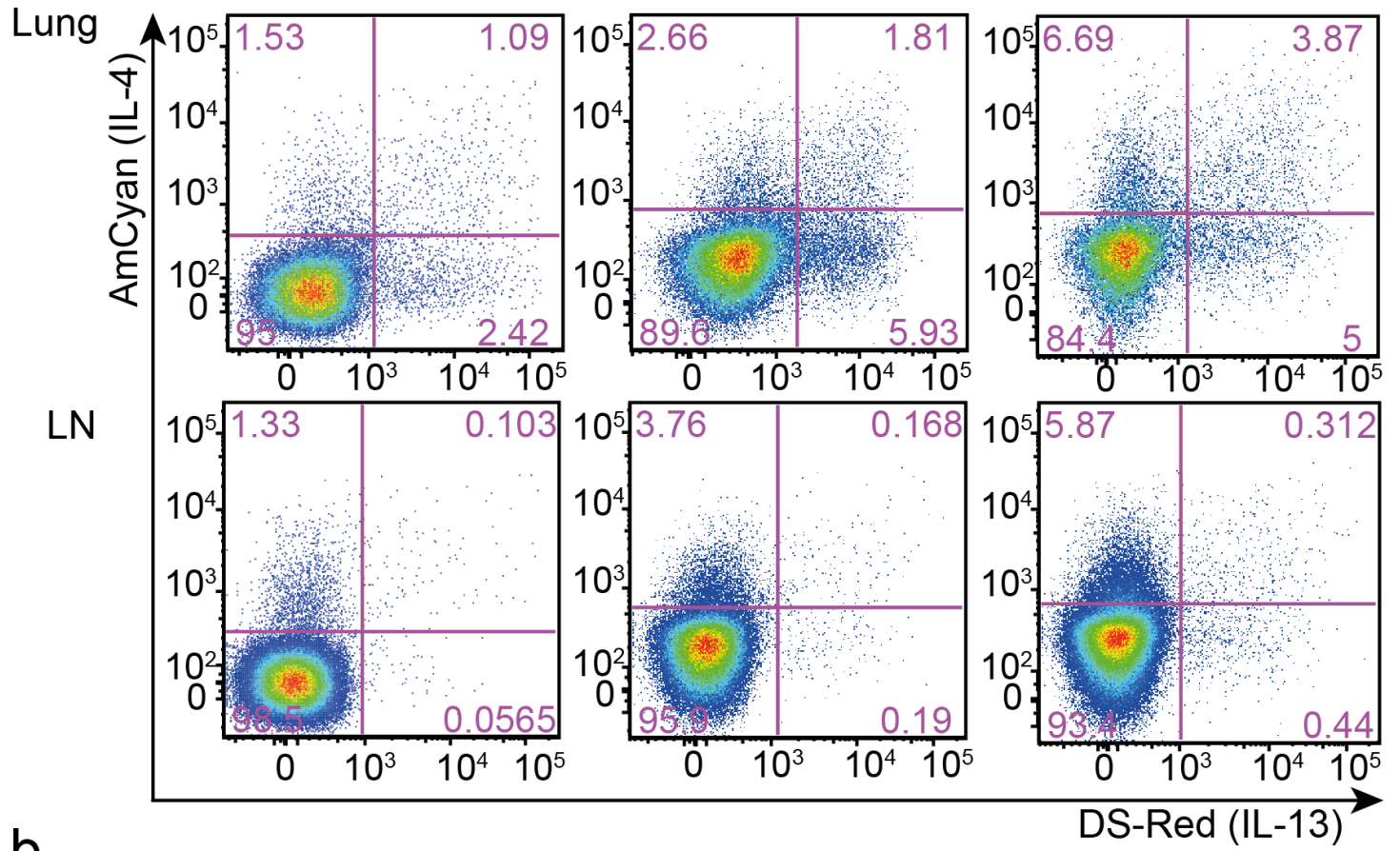

b
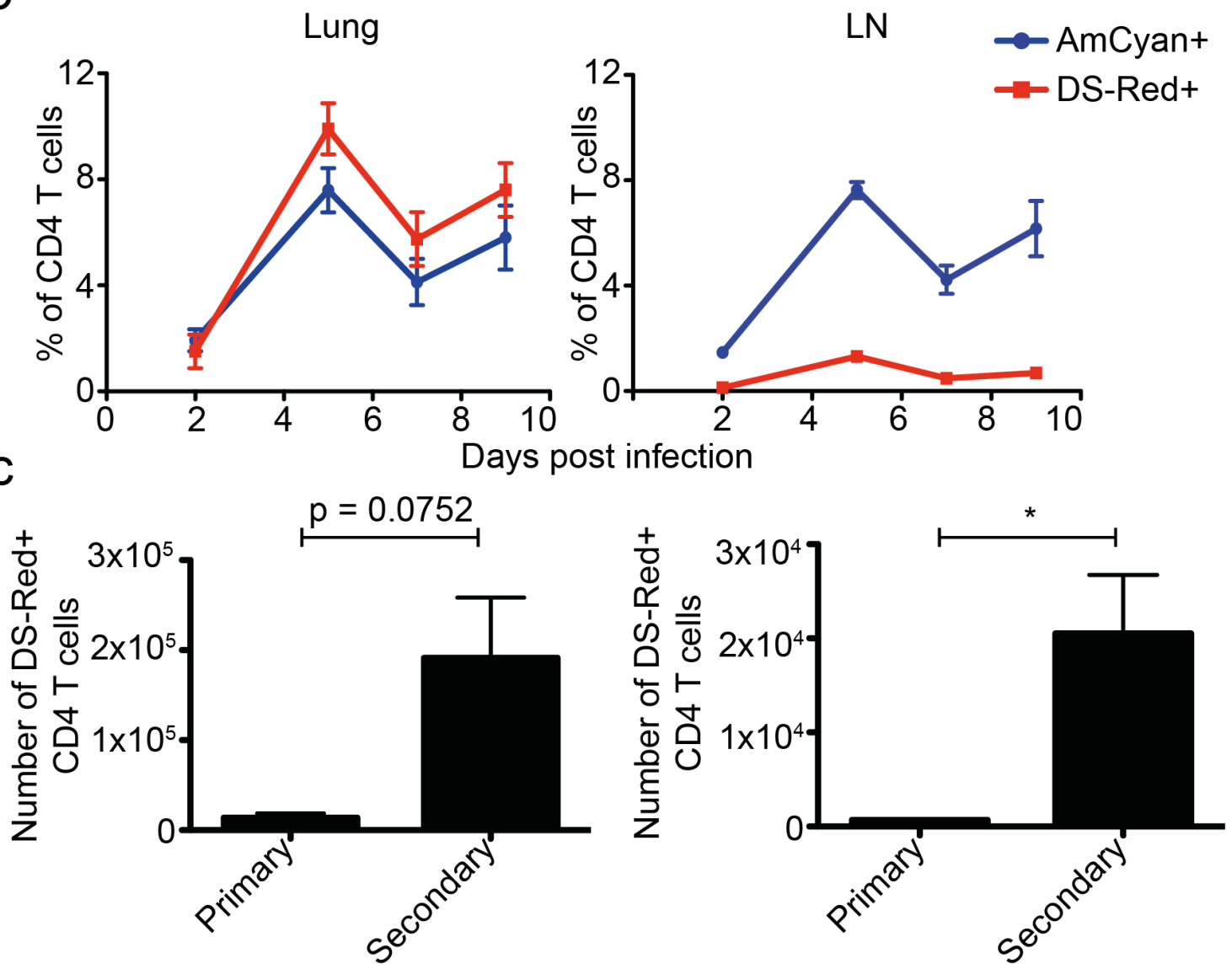

Figure 4.10 - Secondary infection leads to increases in IL-13 reporting CD4 $^{+} T$ cells compared with primary infection.

a. Representative plots and $\mathbf{b}$. proportions of lung and mediastinal LN CD4+ $\mathrm{T}$ cells expressing AmCyan and DS-Red at various time points after a secondary subcutaneous $\mathcal{N}$. brasiliensis infection. Plots show mean \pm S.E.M. $n \geq 3$ in each group. Each graph is representative of two independent experiments. c. Number of DS-Red ${ }^{+} \mathrm{CD}^{+} \mathrm{T}$ cells in the lung and LN at day 6 
post primary and secondary infection. Bar graphs show mean \pm S.E.M. $n=3$ in each group. Data are representative of two independent experiments. Statistics were calculated for each cytokine using Student's t test * $\mathrm{P}<0.05$

\subsubsection{FTY720 blockade of LN egress affects both IL-4 and IL-13 expression by $\mathrm{CD}^{+} \mathrm{T}$ cells}

The mechanism inhibiting IL-13 expression within the LN remained unclear. I hypothesised that Th2 cells within the LN responded to antigen and began expressing IL-4 but do not receive sufficient exposure to antigen in the correct context within the LN to induce IL-13 expression. When the Th2 cells migrate from the LN to effector tissue where they are exposed to antigen again they are permitted to express IL-13. To test this theory reporter expression by $\mathrm{CD}^{+}{ }^{+} \mathrm{T}$ cells within the mediastinal $\mathrm{LN}$ and lung of $\mathcal{N}$. brasiliensis infected 4C13R mice treated with the sphingosine-1 phosphate receptor agonist FTY720 was assessed. FTY720 causes down regulation of sphingosine-1 phosphate receptor 1 on T cells and leads to sequestration within secondary lymphoid organs and inhibition of migration to the periphery. Treatment was begun on day 1 post subcutaneous infection as it has been shown that FTY720 can inhibit migration of APGs to the $\mathrm{LN}^{320,321}$, which could effect the $\mathrm{T}$ cell priming therefore the treatment was delayed to allow migration of antigen loaded APGs to the LN.

Treatment affected IL-4 reporting by the CD4 ${ }^{+} \mathrm{T}$ cells in both the $\mathrm{LN}$ and the lung, though there was no significant decrease in the percentage of $\mathrm{CD} 4^{+} \mathrm{T}$ cells that were $\mathrm{AmCyan}^{+}$. There was a decrease in the number of IL-4 reporting lung CD4 ${ }^{+} \mathrm{T}$ cells when treated with FTY720 (Figure 4.11a). In the LN FTY720 treatment led to an increase in the numbers and percentages of $\mathrm{CD} 4^{+} \mathrm{T}$ cells expressing the IL-13 reporter (Figure $4.1 \mathrm{lb}$ ). Although there was an increase in the percentage of $\mathrm{CD} 4^{+} \mathrm{T}$ cells that were DS-Red ${ }^{+}$, the number in the lung was not altered by treatment. This indicated either that the IL-13 expressing CD4 ${ }^{+} \mathrm{T}$ cells within the lung do not require migration through the $\mathrm{LN}$ for activation, or $\mathrm{LN}$ activated $\mathrm{CD} 4^{+} \mathrm{T}$ cells that go on to express IL13 in the lung are not sequestered by treatment with FTY720. In summary sequestration of T cells within the LN did induce increased IL-13 expression by LN $\mathrm{CD}^{+} \mathrm{T}$ cells, however treatment did not lead to the expected decrease of IL-13 reporting cells within the lung, indicating that in vivo cytokine regulation may be more complicated than originally thought. 

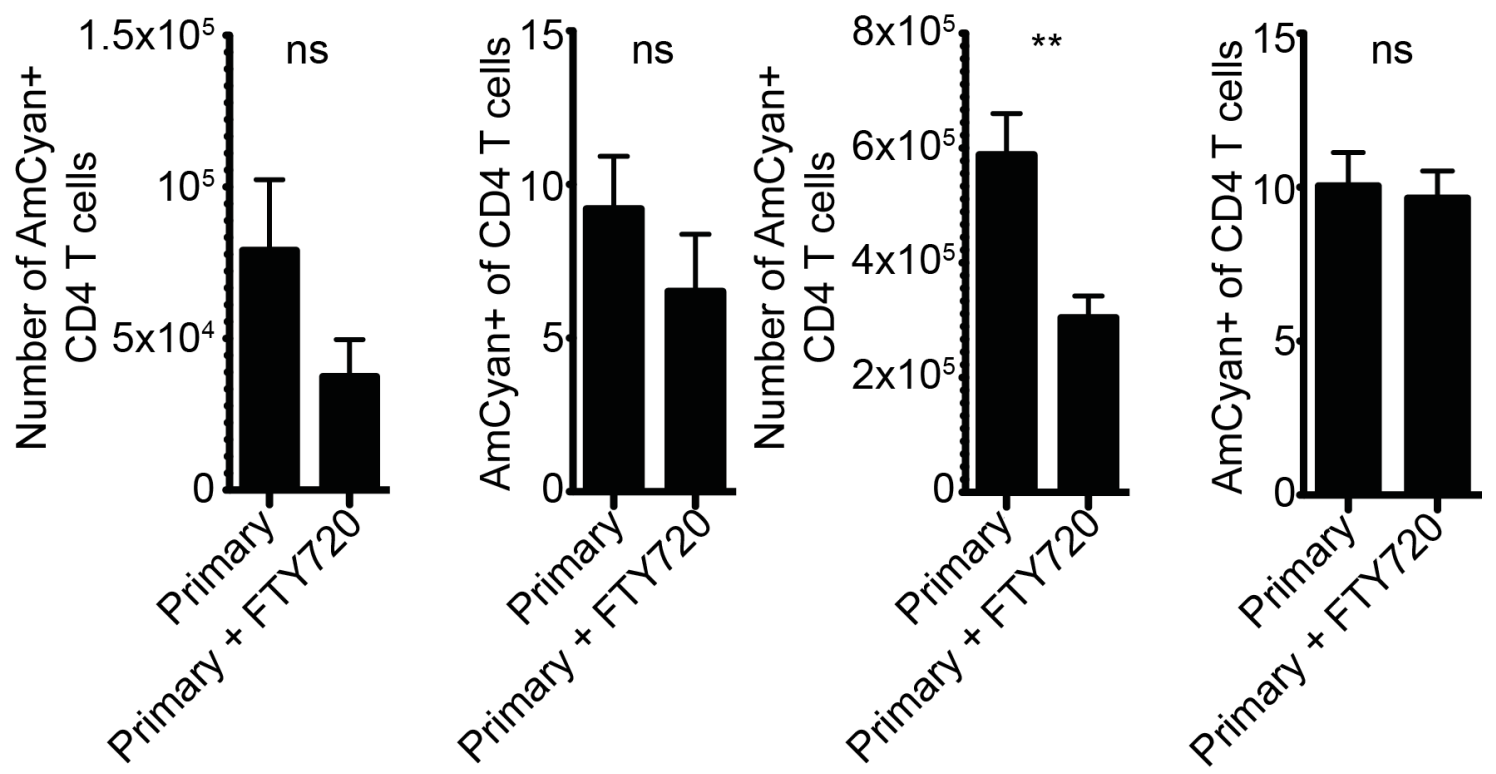

b

$\mathrm{LN}$

Lung
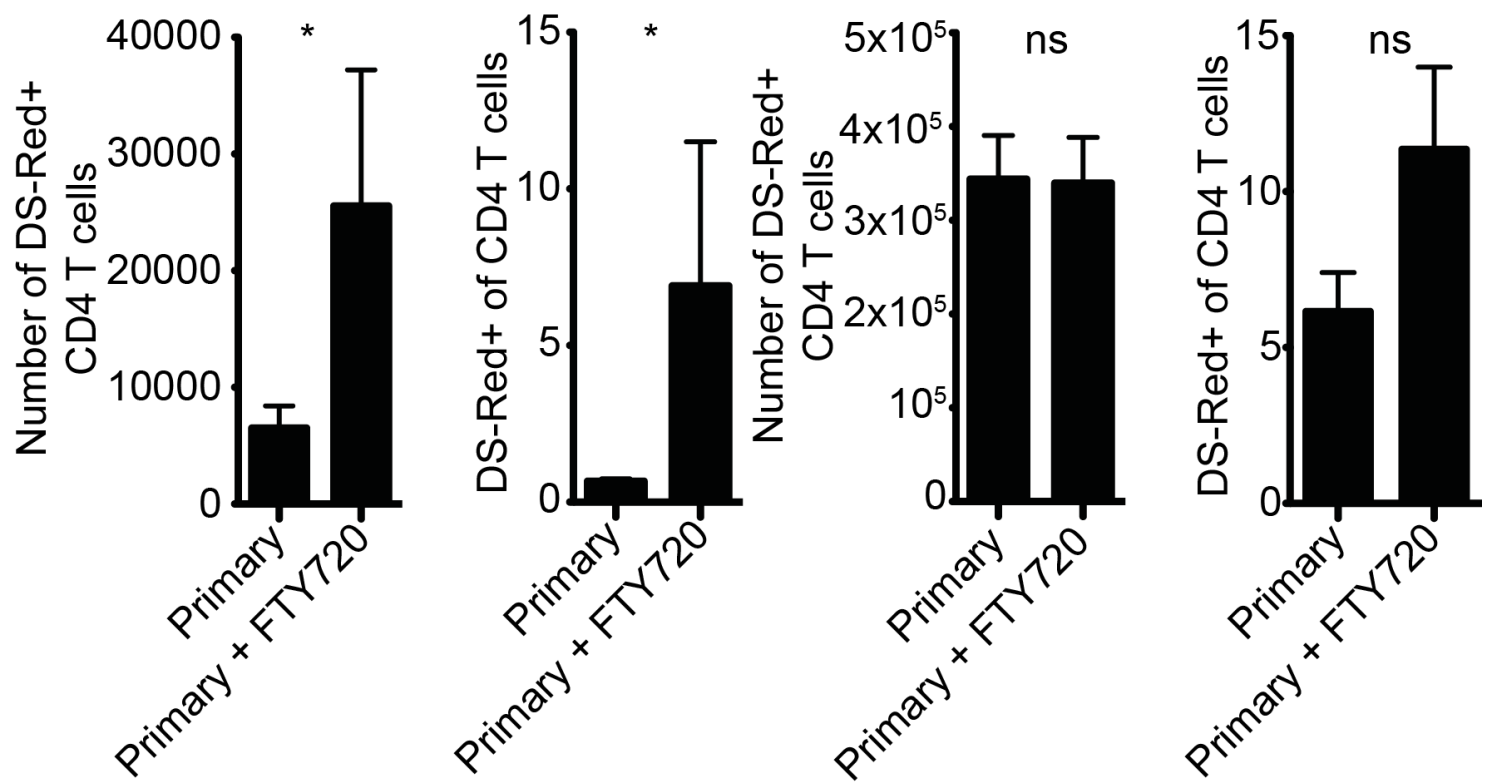

Figure 4.11 - Inhibition of LN egress with FTY720 has differential effects on IL-4 and IL-13 during $N$. brasiliensis infection.

a. Numbers and proportions of IL-4 reporting AmCyan+ CD4 T cells within the LN and the lung on day 9 after a subcutaneous $\mathcal{N}$. brasiliensis infection with and without FTY720. b. Numbers and proportions of IL-13 reporting DS-Red ${ }^{+}$CD4 ${ }^{+}$T cells within the LN and the lung on day 9 after a subcutaneous $\mathcal{N}$. brasiliensis infection with and without FTY720. Bar graphs show mean \pm S.E.M. $\mathrm{n} \geq 3$ in each group. Data are representative of two independent experiments. Statistics were calculated for each cytokine using Student's t test $* \mathrm{P}<0.05 * * \mathrm{P}<$ $0.01 \mathrm{~ns}=$ non significant. 


\subsection{Discussion}

In this chapter I aimed to characterise the mechanisms that mediate $\mathrm{CD} 4^{+} \mathrm{T}$ cell expression of IL-13, and compare them to those that regulate IL-4 production. IL-4 has traditionally been a marker of Th2 cell however results from this work have highlighted the fact that IL-4 and IL-13 are not regulated together, with significant temporal and spatial differences in their expression, indicating that the use of IL-4 as an identifier of Th2 cells may be a limiting approach. Despite IL-4 and IL-13 being structurally similar cytokines the different biologic functions they mediate in vivo potentially explain the disparity in their expression by $\mathrm{CD} 4^{+} \mathrm{T}$ cells.

The delay of IL-13 expression observed in vitro complements the previously published in vivo data that $\mathrm{CD}^{+}{ }^{+} \mathrm{T}$ cells in the LN do not express IL-13. The paper by Liang et al. inferred that it was insufficient expression of GATA3 that prevented IL-13 expression by LN CD4 ${ }^{+} \mathrm{T}$ cells, and that Th2 cells migrated out of the LN before GATA3 levels were high enough to induce IL-13. Tfh cells however remain within the LN and express IL-4. It was proposed that the master transcription factor Bcl-6 inhibited high expression of GATA3, keeping the levels too low to permit IL-13 expression by Tfh cells $^{311}$. Due to technical difficulties I could not assess Bcl-6 levels in the cultured cells so whether low levels of this transcription factor in the newly differentiated Th2 cells inhibited IL-13 expression remains to be determined. LN CD4 ${ }^{+}$T cells express Bcl-6 within two cycles of proliferation ${ }^{98}$, and while there is a brief peak of $\mathrm{Bcl}-6$ expression by $\mathrm{CD}^{+} \mathrm{T}$ cells in Th1 culture it has not been assessed whether this occurs similarly in Th2 cultures $^{322}$.

Whilst Bcl-6 restriction of GATA3 expression might play a role in inhibiting IL-13 expression by LN Th2 cells $^{323}$, the data that sustained or multiple TCR engagement is required indicates a further level of control. It has been shown that Th2 inducing adjuvants, compared with Th1 adjuvants, decrease the amount of time a CD4 ${ }^{+} \mathrm{T}$ cell interacts with DCs in the reactive LN, partially through diminished co-stimulatory receptor expression on the $\mathrm{DCs}^{89}$. Potentially this decreased interaction time also provides an in vivo regulatory mechanism for inhibiting IL-13 expression within the LN. Further studies utilising multiphoton microscopy could shed light whether limited CD4+ T cell-DG interactions are contributing to the prevention of IL-13 expression within the LN. 
I used FTY720 in an attempt to see if trapping CD4 ${ }^{+} \mathrm{T}$ cells within the LN would induce expression of IL-13, or if they required transit to the lung before they could produce the cytokine. The increase in IL-13 expressing $\mathrm{CD}^{+} \mathrm{T}$ cells within the FTY720 treated LN could indicate that the Th2 cells trapped in the LN were there long enough, and stimulated frequently enough to induce IL-13. Interestingly the decrease in the number of IL-4 reporting CD4+ T cells was unexpected; if FTY720 was trapping the cells within the LN and increasing the amount of stimulation the cells were receiving (as was my inference from the IL-13 data) then why the number expressing IL-4 dropped remains unclear.

I had predicted that FTY720 treatment would decrease the number of CD4 ${ }^{+} \mathrm{T}$ cells that entered the lung from the $\mathrm{LN}$, and as expected the total number, and the number of $\mathrm{AmCyan}^{+} \mathrm{CD}^{+} \mathrm{T}$ cells were decreased in the treated lungs (Figure 4.11). Surprisingly the number of IL-13 reporting cells was not altered. IL-13 expression by, and the requirement for, lung-resident $\mathrm{CD}^{+} \mathrm{T}$ cells in protective immune responses against $\mathcal{N}$. brasiliensis has been measured previously ${ }^{134}$. These studies observed a decrease in the total amount of IL-13 secreted by restimulated whole lung cells from FTY720 treated mice, however this could be due to decreased activation of other IL-13 expressing populations such as ILC2s. The discovery that CD4+ T cell IL-13 expression may occur without the requirement of $\mathrm{LN}$ migration is novel and potentially has far reaching consequences for both parasitic infection and allergies.

\subsection{Conclusions}

I have shown that $\mathrm{CD}^{+} \mathrm{T}$ cell expression of IL-13 is differently regulated compared to IL-4. These experiments have demonstrated temporal and spatial regulation of IL-13 compared to IL-4 by recently activated CD4 ${ }^{+} \mathrm{T}$ cells. IL-13 is expressed much later than IL-4 in Th2 differentiating culture, and within the LN CD4+ T cells do not express IL-13 at all. By inhibiting egress of the $\mathrm{CD} 4^{+} \mathrm{T}$ cells from the $\mathrm{LN}$ during $\mathcal{N}$. brasiliensis infection IL-13 expression can be induced. LN trapping of CD4 ${ }^{+} \mathrm{T}$ cells however does not change the number of IL-13 expressing CD4+ $\mathrm{T}$ cell within the lung indicating that IL-13 expression can potentially be activated outside of lymphoid tissue. I have discovered that IL-13 is differentially expressed to IL-4 and while these cytokines are related, they are under the control of very different regulatory mechanisms. These data can inform future studies particularly those aiming to specifically target IL-13 in an attempt to inhibit its pathogenic roles in allergic and fibrotic diseases. 


\section{Innate immune cells of the skin display divergent expression of Type 2 cytokines}

\subsection{Introduction}

The skin offers a physical barrier to exclude potential pathogens and it contains many resident populations of immune cells that mediate rapid responses to breaches in the barrier and attacks by opportunistic infections. Inappropriate immune responses in the skin can alter function and structure of the tissue as is seen in skin inflammatory disorders including psoriasis ${ }^{324}$ and atopic dermatitis ${ }^{325}$. The immune components that facilitate the pathology of these skin diseases are not fully characterised and this chapter attempts to focus on two immune cell types and their contributions to skin immune responses.

Populations of ILCs have been identified in other barrier sites including the gut ${ }^{254}$ and lungs ${ }^{116}$ however prior to this study whether a skin resident population exists, and what functions these cells might have had not been assessed. I have described a novel population of dermal resident ILCs discovered in collaboration with researchers from the Centenary Institute in Sydney ${ }^{265}$, and I have assessed the function of these cells in a range of inflammatory models. 
MC903, also known as calcipotriol, is a derivative of the vitamin D3 metabolite calcitriol and is traditionally used as a treatment for psoriasis ${ }^{326}$. Topical, daily application of MC903 causes localised skin inflammation that in a mouse phenotypically reproduces the salient characteristics of atopic dermatitis, with hyperproliferation of keratinocytes, infiltration of immune cells into the dermis and oedema at the treated site. The pathology is dependent upon TSLP and is associated with increased serum $\operatorname{IgE}$ and Th2 responses, though the pathogenic changes in the skin are independent of antibody or $\mathrm{T}$ cell responses as they can occur in Rag1-/- animals. ${ }^{297}$ In this chapter I have described ILC responses during the MC903 model then characterised basophil responses, assessing their cytokine production during treatment and the factors that mediate their activation and recruitment. Together these data assess the contributions to skin inflammation by two cell populations, one resident and one recruited and compared their differential responses and cytokine expression patterns.

\subsubsection{Aims}

In this chapter I aimed to characterise a population of novel innate immune cells within the skin and assess their responses in a range of inflammatory conditions. IL-4 and IL13 producing cells in the skin were characterised using reporter mice and, utilising the MC903 model of skin inflammation, I compared the responses of ILCs and basophils. Specifically these experiments aimed to:

- Characterise the dermal innate lymphoid cells (dILCs)

- Compare IL-13 production by dILCs in mice across a range of ages and in inflammatory conditions

- Assess the susceptibility of skin immune cells to antibody depletion

- Measure basophil responses in a vitamin D analogue driven model of skin inflammation.

\subsection{Results}

\subsubsection{Dermal innate lymphoid cells are a distinct population of skin resident immune cells}

Roediger et al. discovered a population of cells within the skin that phenotypically resembled ILCs, it was decided that I would assess the potential role of these cells in models of Type 2 inflammation of the skin ${ }^{265}$. A gating strategy was defined to identify 
the dermal ILC (dILC) population of the skin. To prepare a single cell suspension for flow cytometry the skin needed to be digested with a buffer containing type IV collagenase and DNase type I and then ground through a metal mesh. Using CD45 to identify the haematopoietic cells that make up roughly $5 \%$ of the digested skin cell suspension, lymphoid cells were identified by CD90 expression and myeloid cells excluded using CD11b. To exclude T cells and NK cells from the innate lymphoid gate, cells identified as CD3-, NK1.1- and CD2- were gated on (Figure 5.1a). This population of non-T, non-NK lymphoid cells, making up around $2.5 \%$ of the CD45 ${ }^{+}$cells in the skin (Figure 5.1b), were classified as the dermal innate lymphoid cells (dILCs). 
a
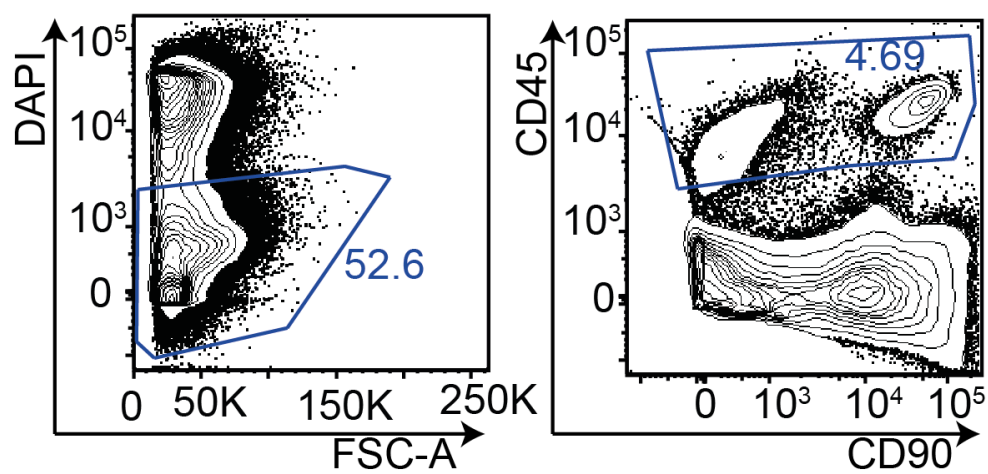

b
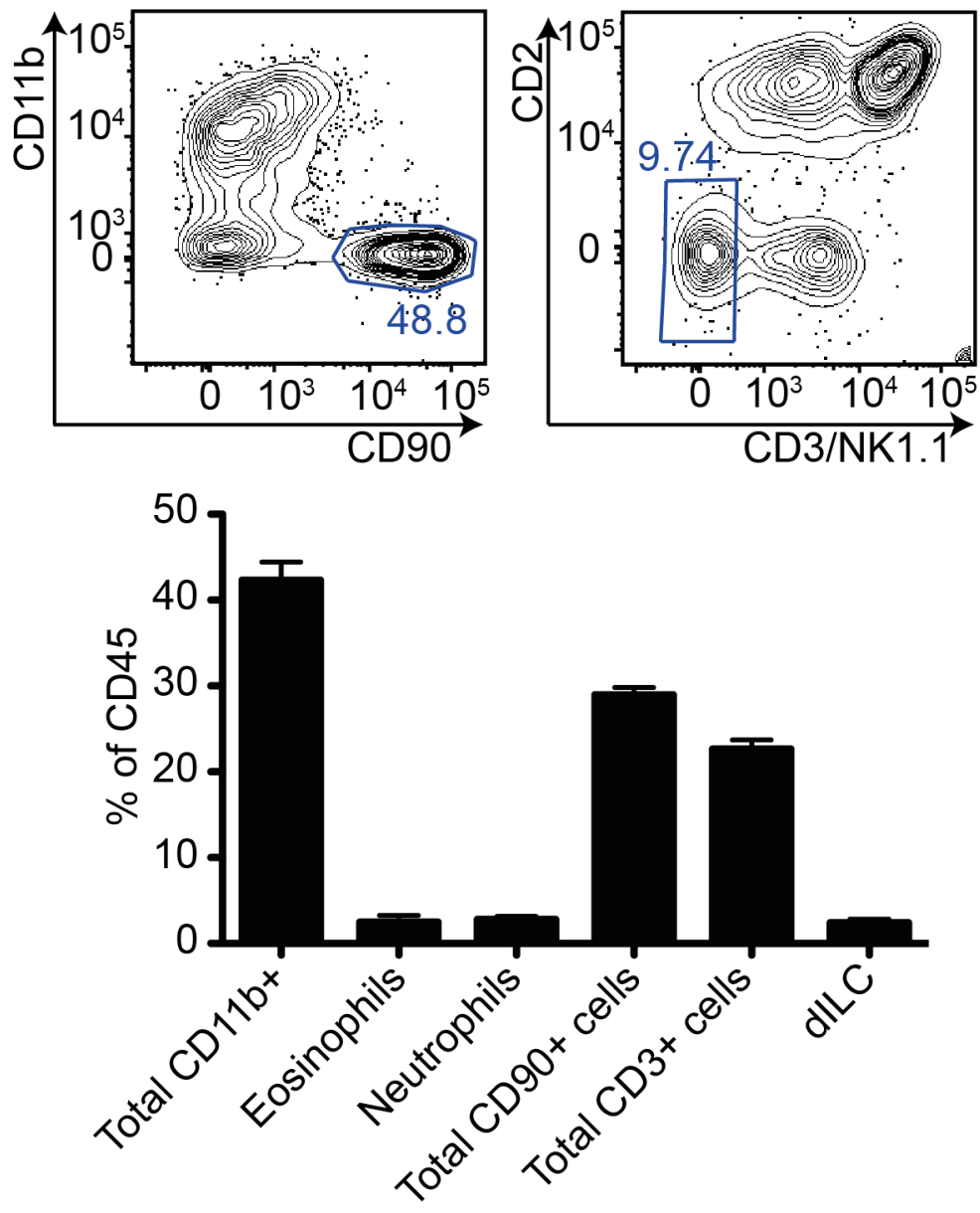

Figure 5.1 - dILGs are a unique population of skin immune cells.

a. Gating scheme identifying dermal innate lymphoid cells (dILCs). b. Proportion that different cellular populations make up of $\mathrm{CD} 45^{+}$cells within the total ear skin of naïve mice. Bar graphs show mean \pm S.E.M. $\mathrm{n} \geq 8$ in each group. Data was pooled from 4 independent experiments. 
The dILCs were phenotyped by flow cytometry for various cell surface molecules, looking for expression of ILC related receptors and ensuring the lack of expression of molecules associated with other cell populations (Figure 5.2). Almost all the dILCs expressed CD44 and the majority of cells were positive for GD69 and the IL-7R (CD127), variable levels of Scal (Ly6E) and inducible T cell costimulator (ICOS) were also detected, all cell surface molecules previously described to be expressed by ILCs ${ }^{251}$. Expression of GD11a, CD34, CD95 and CD103 were also detected. It has since been shown that CD103 expression was limited to dermal ILCs, it was not detected on ILC populations from any other tissue ${ }^{265}$. A population of IL-33R ${ }^{+}(\mathrm{T} 1-\mathrm{ST} 2)$ and a low percentage of $\mathrm{CD} 25^{+}$(IL-2R $\alpha$ ) cells were also observed. Both receptors had been associated on ILC2s in other tissues allowing them to response to IL-33 and IL-2253. dILGs were found to be negative for the myeloid associated cell surface molecules CD11c, CD80, CD86, the canonical eosinophil molecule siglec F, antibody receptors FceRI and CD16/32 (Fc $\gamma$ RII and Fc $\gamma$ RIII), CD154 (CD40Ligand), CD152 (CTLA-4), mast cell expressed stem cell growth factor receptor CD117 (G-Kit), T cell co-receptors CD4 and CD8, Gr1 (Ly6C/Ly6G), KLRG1 and CD121b (IL-1Rb). Notably IA/IE (MHC class II) was not detected which had been measured on populations of ILCs in other tissues ${ }^{254}$.

Using this gating strategy I was able to specifically identify a novel population of skin cells that appear to resemble ILCs, although they express many of the common ILC associated cell surface molecules they were not clearly positive for all markers traditionally associated with ILC2s. 

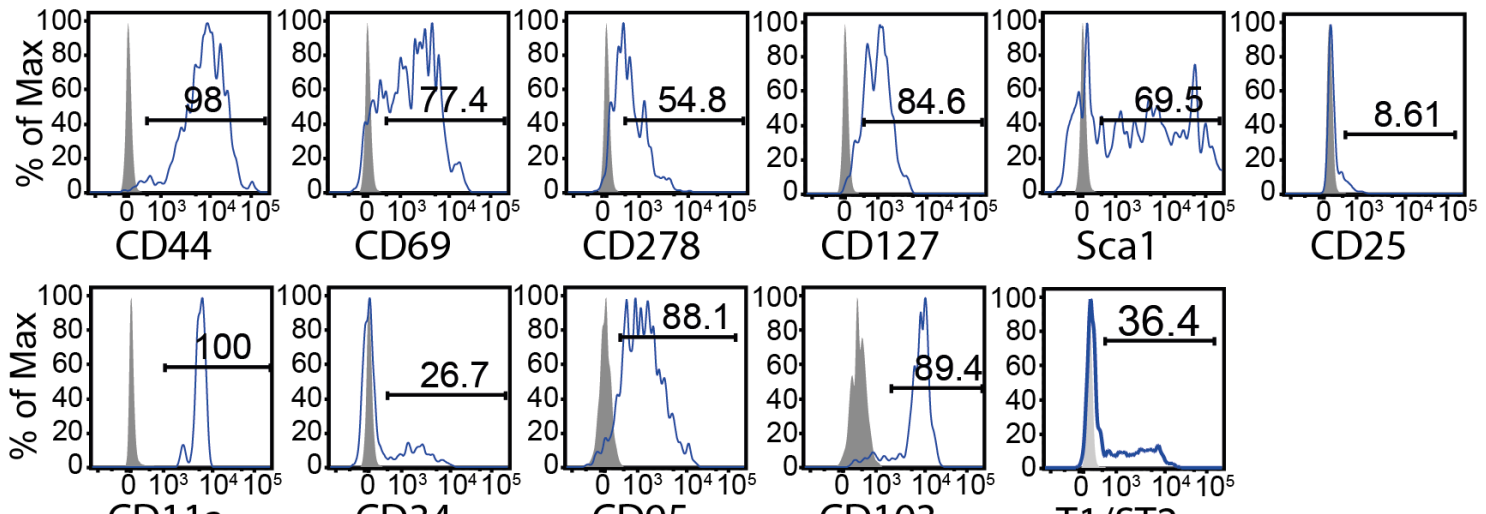

Sca1
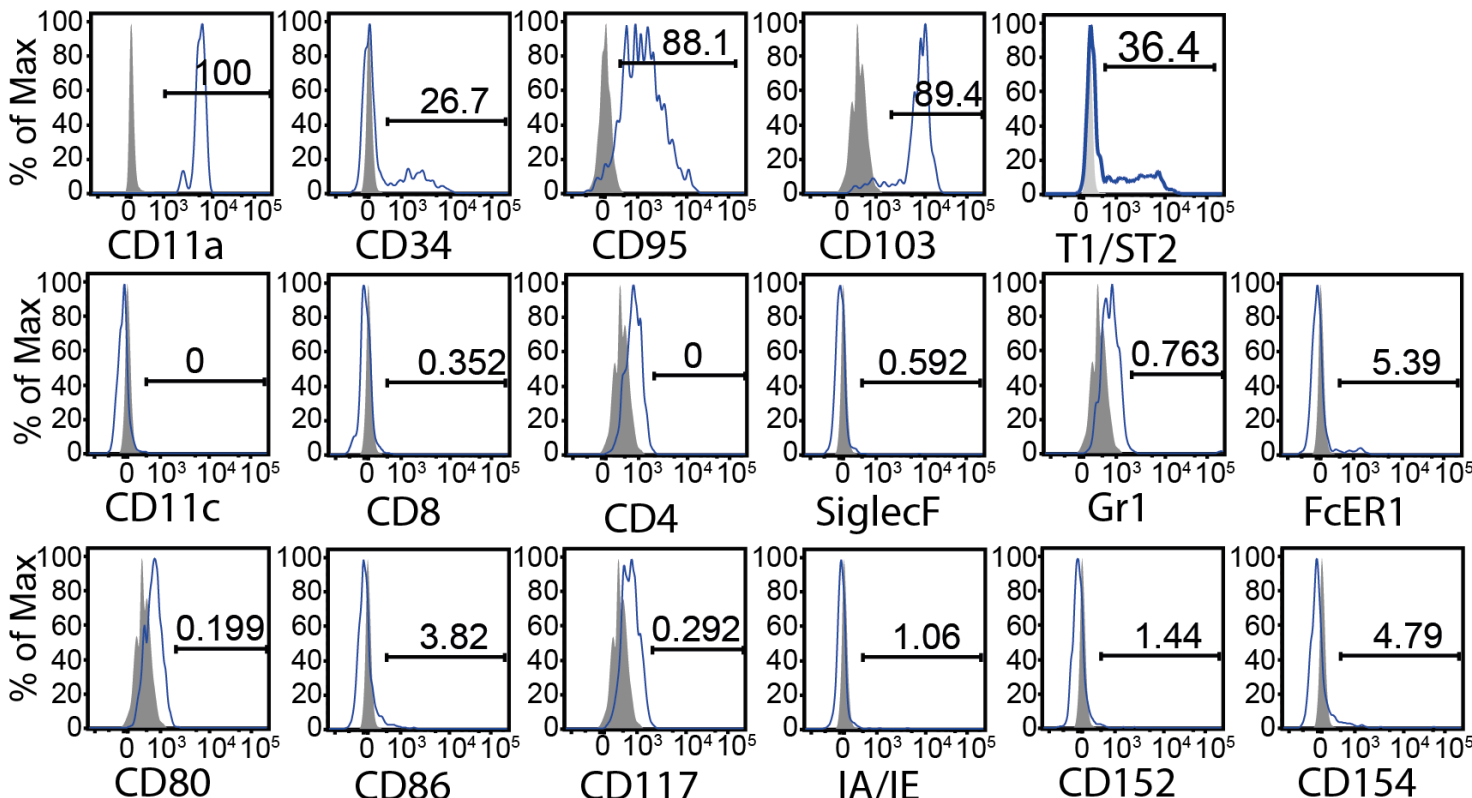

SiglecF

Gr1

FCER1
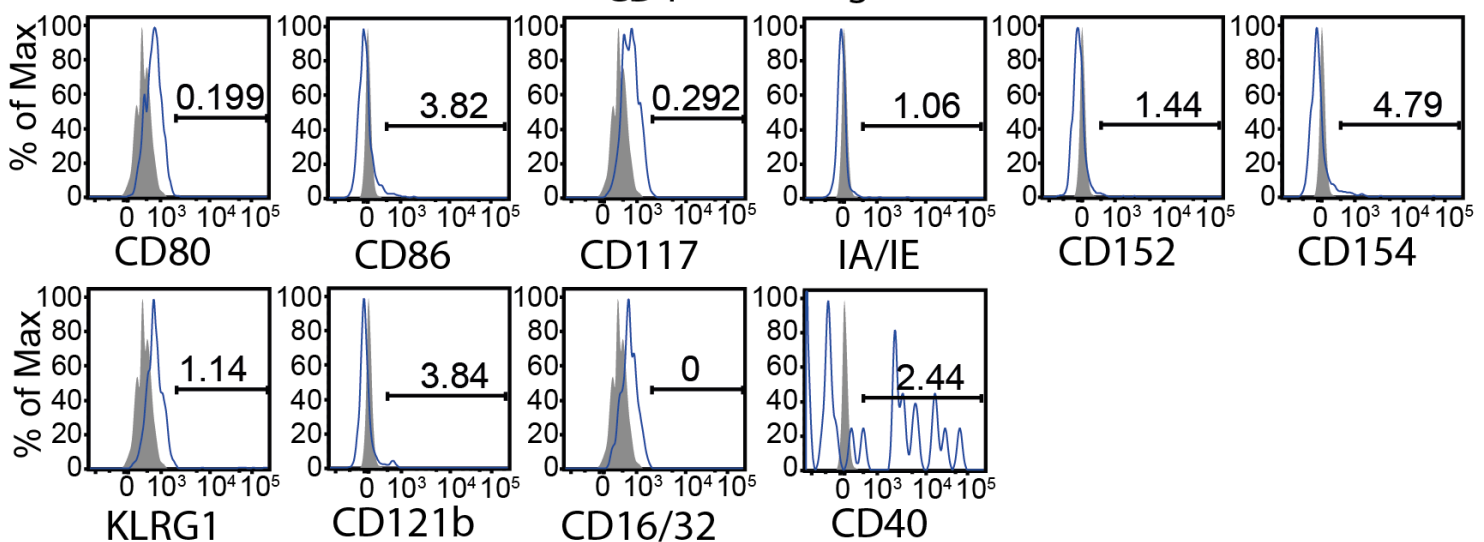

Figure 5.2 - dILGs have a phenotype characteristic of ILGs from other tissues.

a. Histograms demonstrating expression of a range of cell surface molecules on dILCis gated as in Figure 5.1a. Gates are set upon isotype controls and numbers indicate percentage of dILC population that fall within the gate. Plots are representative of triplicates and two independent experiments. 


\subsubsection{IL-13 is constitutively expressed by dermal innate lymphoid cells}

dILCs in the skin of 4C13R mice were assessed at baseline for their expression of IL-4 and IL-13 and a population of DS-Red expressing cells reporting IL-13 expression were identified in naïve skin (Figure 5.3). The DS-Red cells were all CD45 ${ }^{+}$and CD90 ${ }^{+}$and the majority of DS-Red ${ }^{+}$cells were from the CD3-and NK1.1- population. Some minor expression from the CD $3^{\text {hi }} \mathrm{NK} 1.1^{\text {hi }}$ cells was detected as well, although this population is smaller than the CD3- NK1.1- DS-Red ${ }^{+}$cells (Figure 5.3a). When dILCs were specifically gated a population of cells were consistently observed with higher DS-Red ${ }^{+}$ expression than background (Figure 5.3b), however these cells did not report any IL-4 expression as there was no detection of AmCyan above background (Figure 5.3c).

It was noted that the age of the mouse made a large difference on the proportion of dILGs that were IL-13 reporter positive (Figure 5.4a). Very young mice less than 4 weeks of age had about $66 \%$ of their dILCs expressing DS-Red while older mice, between 4 and 8 weeks, had about 44\% DS-Red ${ }^{+}$dILGs. A further non-significant reduction in the proportion of IL-13 reporting dILGs was observed in mice older than 8 weeks of age (Figure 5.4b).

Thus despite a low proportion of dILGs expressing T1/ST2 (IL-33R) and CD25 (IL$2 \mathrm{R} \alpha$ ), receptors usually associated with ILC2s, a proportion of the dILCs expressing the IL-13 reporter were detected. This indicated that at least some dILCs are of a group 2 phenotype in naive skin. Furthermore I found that the proportion of the dILCs that constitutively express IL-13 decreases as the mice mature. 
Non-transgenic littermates

a

Live CD45+ CD11b-CD90+

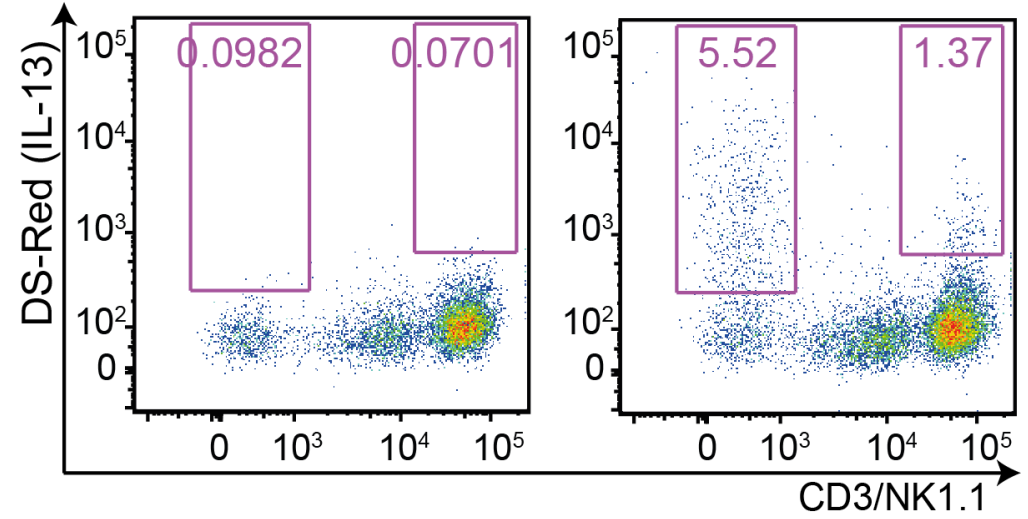

b Live CD45+ CD11b-CD90+CD3-NK1.1-

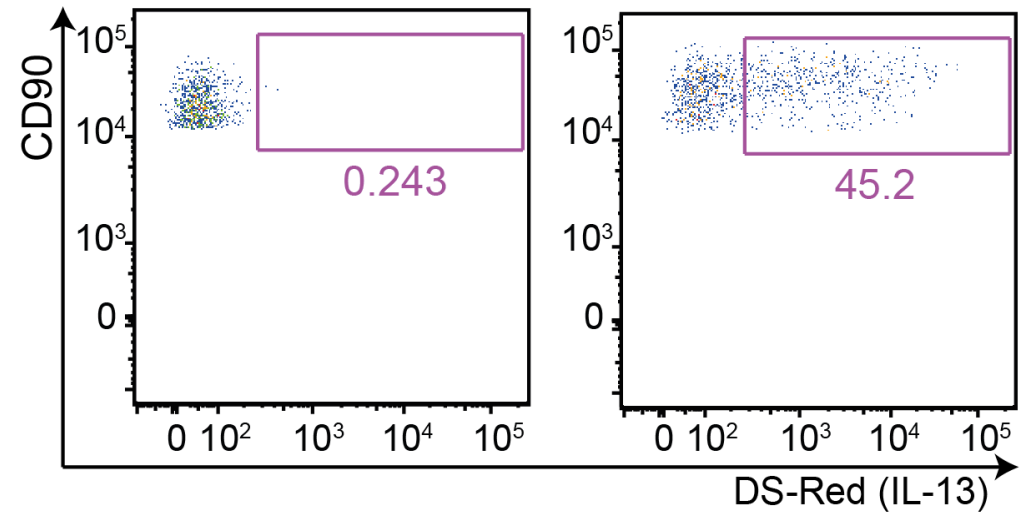

C Live CD45+ CD11b-CD90+CD3-NK1.1-

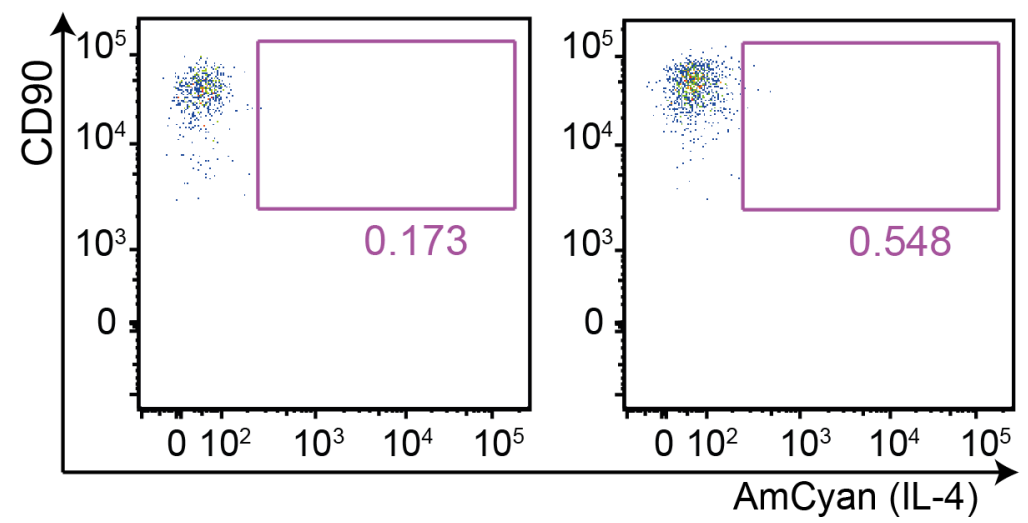

Figure 5.3 - dILGs constitutively express IL-13 but not IL-4.

Representative plots of DS-Red expression on a. Total lymphocytes $\left(\mathrm{CD} 45^{+} \mathrm{CD} 11 \mathrm{~b}-\mathrm{CD} 90^{+}\right)$ and b. dILCs from ear skin from 4C13R and non-transgenic littermate control mice. Plots are representative of many experiments. c. Representative plots of AmCyan expression on dILCs from ear skin of $4 \mathrm{C} 13 \mathrm{R}$ mice. Plots are representative of greater than five independent experiments. 
a

Live CD45+ CD11b-CD90+CD3-NK1.1-
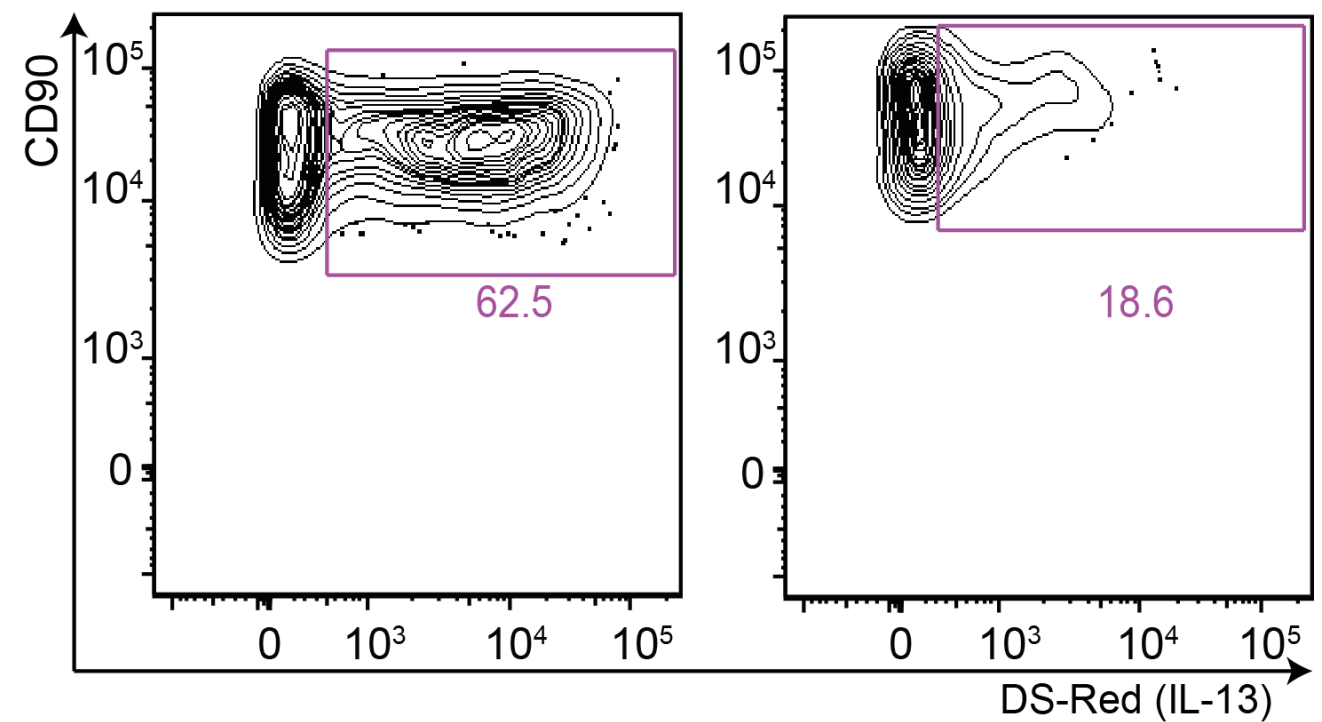

b

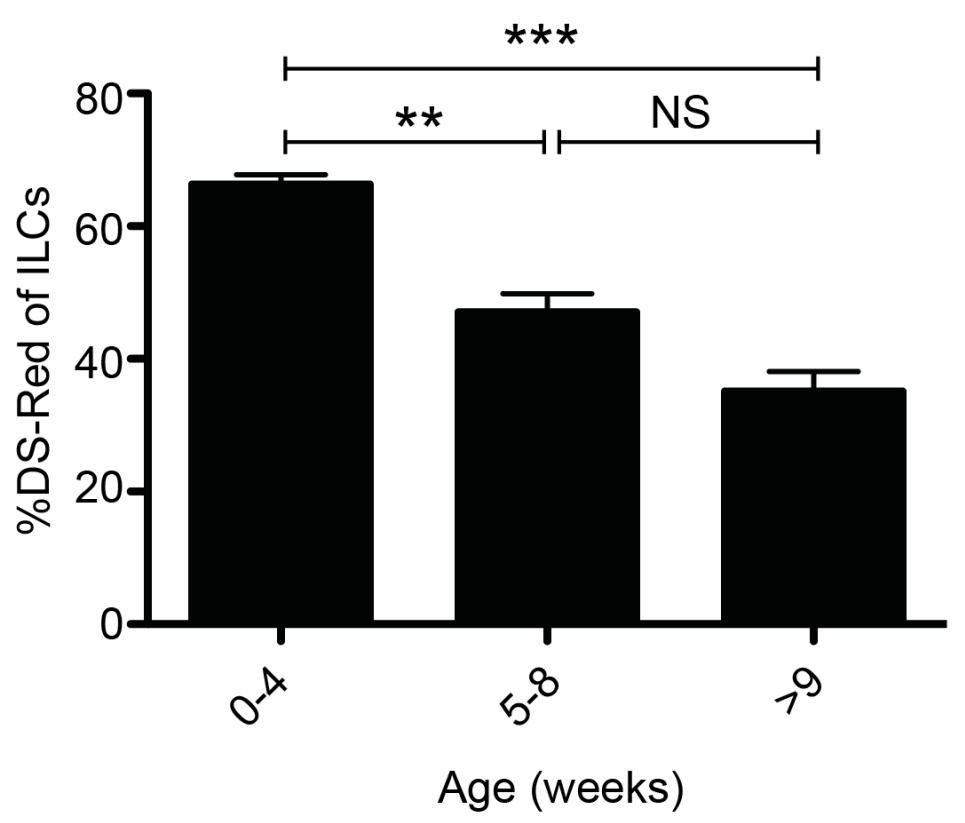

Figure 5.4 - IL-13 expression varies with age.

a. Representative plots of DS-Red expression on dILCs from 2 week and 16 week aged mice. $\mathbf{b}$. Proportion of ear skin dILCs expressing DS-Red from mice grouped into three groups based upon age. Graph shows mean \pm S.E.M. of each group. Groups contain at least seven replicates. Data are representative of two independent experiments. Statistics were calculated using Kruskall-Wallis with Dunn's post-test, **p $<0.01, * * * p<0.001$ 


\subsection{3 dILCs only display minor responses in models of skin inflammation}

IL-13 can contribute to fibrosis and pathology of atopic dermatitis ${ }^{12,} 327$ so we hypothesised that dILCs could contribute to models of skin inflammation through their production of IL-13. Mice were treated to assess dILC responses in a skin specific model of inflammation, a model that induces the Type 2 associated $\operatorname{IgE}$ antibody. However the model required optimisation to meet our ethical guidelines and inflammatory requirements. Mice were treated with MC903 at the same concentration as the original publication, 4nmol of MC903 dissolved in ethanol per ear daily ${ }^{297}$. The ear skin of these mice clearly became inflamed, with the ears becoming thickened from dermal infiltration of inflammatory cells as well as hyper-proliferation of keratinocytes, the mice became ill by day 7 of treatment, with visible hunching, loss of sleekness to the fur and by day 9 all the mice had to be culled as their weight loss was greater that ethical cut offs. I found that the 17-day model could not be ethically replicated in our laboratory using the same concentration described in the original publication.

To assess the limits of the MC903 model mice were treated with a titration of the drug, treating groups of mice on both ears with 4, 2 or 1 nmol MC903/ear/day, and also treating one group with $4 \mathrm{nmol}$ daily on one ear and ethanol on the other, all these treatments were compared to mice treated daily on both ears with ethanol. Regardless of the concentration of MC903 used to treat, the mice ears thickened at an equal rate (Figure 5.5a). The ears that were only treated with ethanol, even when the contralateral ear was receiving MC903, did not show any thickening (Figure 5.5c). However as observed in the original experiments mice treated on both ears with 4nmol MC903/ear/day succumbed the quickest to the sickness caused by MC903 treatment (Figure 5.5b). This was followed by the mice receiving $4 \mathrm{nmol} /$ day to a single ear, which lost weight at a similar rate to the mice receiving $2 \mathrm{nmol} /$ ear/day on both ears (both groups of mice received a total of $4 \mathrm{nmol} /$ day). Mice receiving $1 \mathrm{nmol} /$ ear/day only began losing weight on day 15 of treatment and all mice in this group survived to the day 17 time point with massive ear swelling (Figure 5.5a and b). 


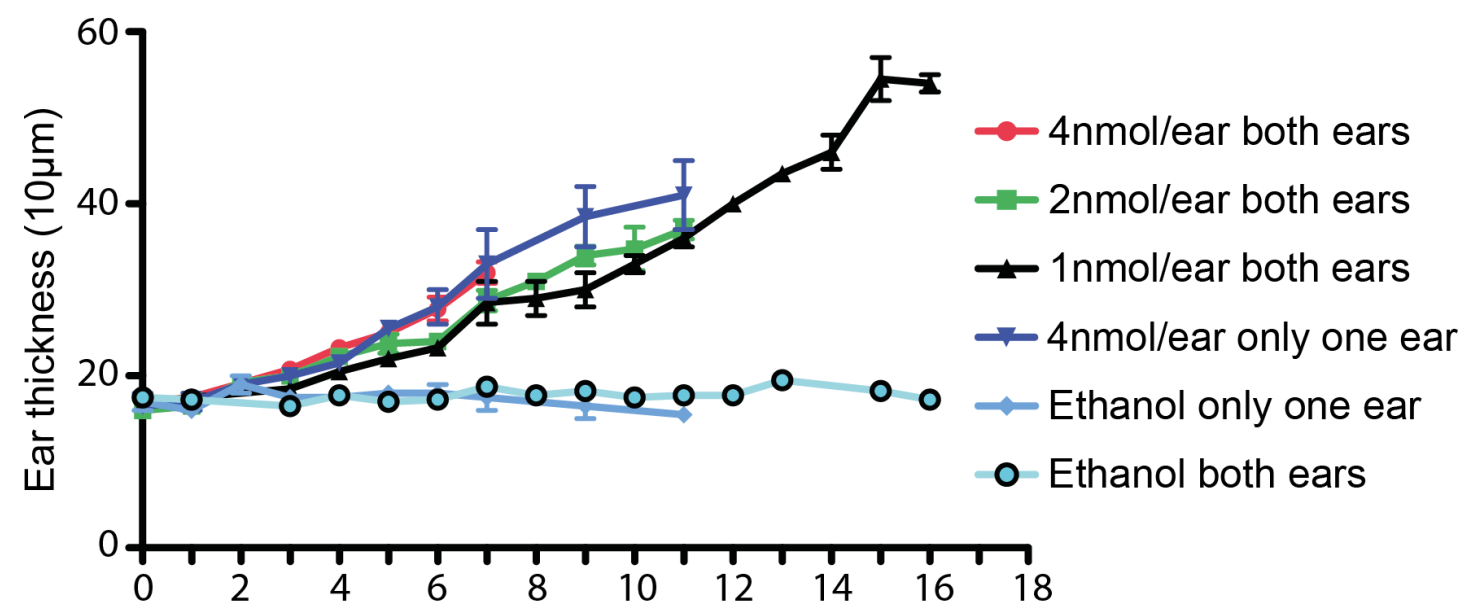

b
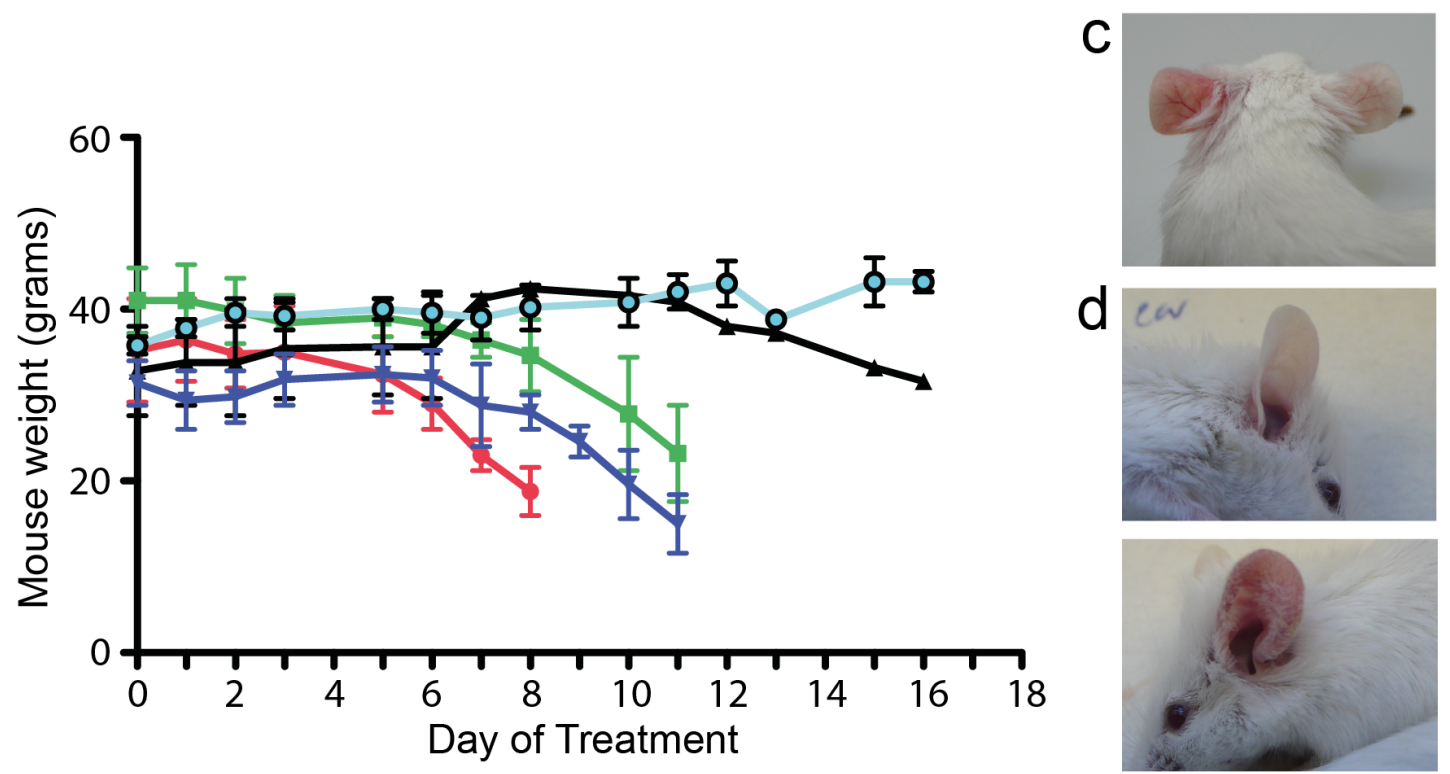

Figure 5.5 - Galcipotriol (MC903) causes inflammation and thickening of skin tissue.

a. Ear thickness measurements of Balb/c mice treated with $10 \mu \mathrm{L} /$ side of ear $(20 \mu \mathrm{L} /$ ear $)$ daily with different concentrations of MC903 or ethanol control. b. Weights of mice undergoing treatment with MC903. Pictures taken of the mice treated on the left ear with $4 \mathrm{nmol} /$ ear MC903 and on the right ear treated with ethanol on c. day 6 and d. day 12 of treatment. Each time point contains two mice, four ears. 
Massive ear thickening, and survival of the mice until at least until day 17, could be achieved if the dose of MC903 was dropped to a quarter of what was originally published $^{297}$. Due to the fact that similar thickening was measured at day 7 with all the doses trialled, I decided to treat with the highest dose trialled, 4nmol/ear/day MC903 as I reasoned that high doses would be most likely to generate responses from the dILCs. The model chosen was to measure the responses of dILCs using the original $4 \mathrm{nmol} /$ day/ear regime at day 7 of treatment.

dILC responses to MC903 treatment were minimal, with no difference in their total number between a 7 day ethanol and $4 \mathrm{nmol} /$ ear/day treated ear (Figure 5.6a). The proportion dILGs constituted of live (Figure 5.6b) and CD45 ${ }^{+}$cells (Figure 5.6c) were diminished in the MC903 treated skin. These data indicated that dILCs were not infiltrating into the skin or proliferating in response to treatment, in fact decreased proportions indicated that other immune cells were increasing in the skin. Treating 4C13R mice with MC903 allowed measurement of the IL-13 reporting from the dILCs. MC903 treatment caused increased numbers (Figure 5.6d) and a statistically insignificant increase in proportions (Figure 5.6e) of DS-Red ${ }^{+}$dILCs in the ear skin above ethanol treated mice. So although there appeared to be slight increases in the activation of dILCs in response to MC903, there was no increase in cell number and the changes observed were not significant so it did not appear that dILCs are very responsive to MC903 treatment. 
a

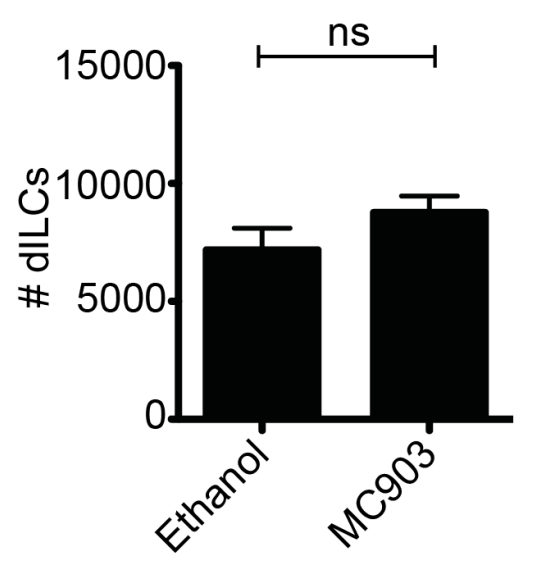

d

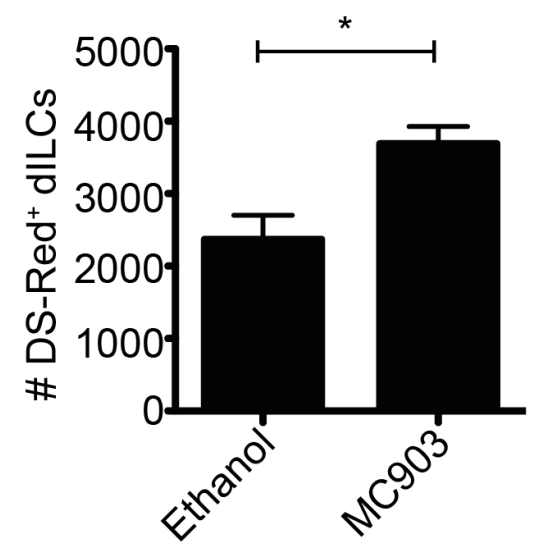

b

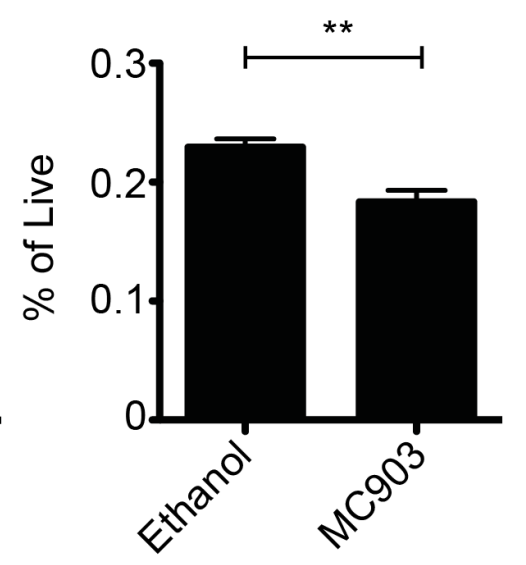

C

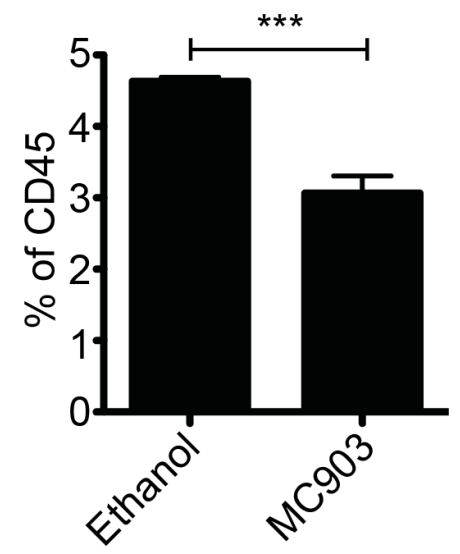

e

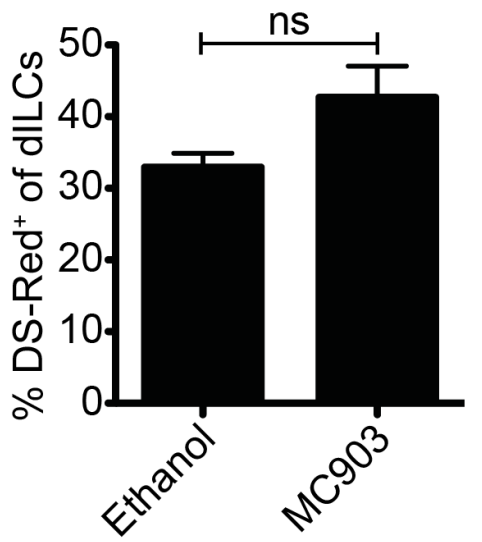

Figure 5.6 - ILGs only show moderate responsiveness in a MC903 model of skin inflammation.

Comparing ear skin responses of MC903 treated and ethanol control 4C13R mice at day 7 of treatment. Measuring; a. numbers of dILCs. b. proportion of dILC of total live cells, c. proportion of dILCs of CD45 ${ }^{+}$cells, d. number of DS-Red ${ }^{+}$dILCs and e. proportion of dILGs expressing DS-Red. Bar graphs show mean \pm S.E.M. for each group. Data are representative of two independent experiments. Statistics were calculated for each group using Student's t test $* \mathrm{P}<0.05 * * \mathrm{P}<0.01 * * * \mathrm{P}<0.001 \mathrm{~ns}=$ non significant. 
dILC did not respond to MC903 in any detectable way, so other inflammatory stimuli were tested to see if these could effect dILC numbers. I hypothesised that the 7 days of the MC903 model may be too long and early dILC responses were be being missed. So I decided to look at early responses after tape stripping or intradermal injection of HDM, IL-33 or TSLP. Tape stripping required repetitively sticking tape to the ears of the mice and pulling it off ${ }^{298}$, the process was repeated 20 times on each ear, removing the cuticle and causing considerable inflammation. The dILG responses 24 hours after treatment were assessed. No differences were found in the numbers of dILC or their proportion of $\mathrm{CD}_{4} 5^{+}$cells (Figure 5.7a). When the dILCs responding 24 hours after intradermal injection of HDM were assessed, similar patterns were observed as with MC903 treatments. HDM injection reduced the proportion of CD45+ cells made up by the dILGs but the total numbers of dILCs per ear did not change between PBS and HDM injection, indicating that while HDM induced some inflammation it wasn't causing dILGs proliferation (Figure 5.7b). So in the 3 inflammatory systems assessed dILCs were not observed responding in changes in cell number.

ILCs have been reported to respond strongly to a range of cytokines, particularly the barrier-derived cytokine IL-25, IL-33 and TSLP ${ }^{328}$. I hypothesised that dILCs would also respond to recombinant cytokine, so IL-33 and TSLP were injected intradermally into 4C13R mice and dILC responses assessed 24 hours later. No changes in the proportion of CD45 or numbers of dILCs were observed, nor in the proportion of dILCs expressing DS-Red compared to the contralateral ears injected with PBS (Figure 5.7c). So unlike ILCs from the lungs or intestines, dILCs did not seem to respond very rapidly to direct cytokine treatment. 

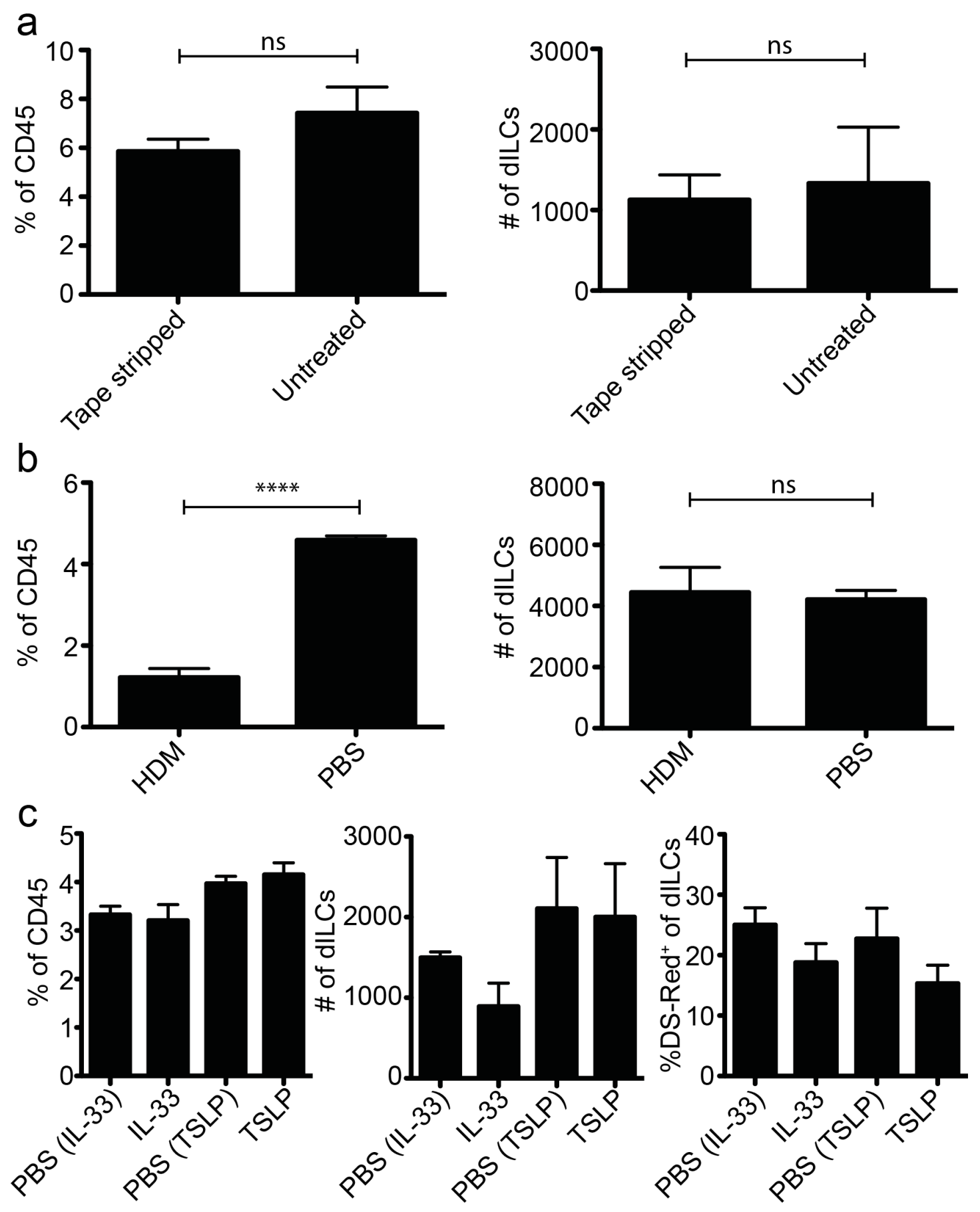

Figure 5.7 - dILGs show marginal responses to various inflammatory stimuli.

a. Proportion of CD45, and numbers, of dILCs in untreated and tape-stripped Balb/c mice 24 hours after treatment. b. Proportion of CD45, and numbers, of dILCs in response to intradermal injection of PBS or HDM in Balb/c mice 7 days after treatment. c. Proportion of CD45, and numbers of dILCs, and proportion of DS-Red ${ }^{+}$of dILCs 24 hours in the ears of 4C13R mice after intradermal injection of the cytokines IL-33 or TSLP compared to contralateral ears treated with PBS. All graphs show mean \pm S.E.M. for each group. Data are representative of two independent experiments. Statistics were calculated for each group using Student's t test to compare against the relevant control $* * * * \mathrm{P}<0.0001 \mathrm{~ns}=$ non significant. 
From these experiments it can be interpreted that dILCs are a quiescent skin resident cell population, not responding via proliferation or IL-13 expression to a range of inflammatory models or to direct cytokine injection. Depletion of dILCs would allow assessment of their contribution to inflammatory models such as MC903 in ways that hadn't yet been detected. MC903 induced inflammation and ear thickening had been documented to be $\mathrm{T}$ cell independent and occur in Rag-/- mice ${ }^{297}$, so if CD90 ${ }^{+}$cells could be depleted from the skin of a Rag1/-- mouse, the role of dILGs could be assessed, NK cells would also be depleted but this could be controlled for with NK cell specific depletion. However I found that regular antibody depletion protocols didn't deplete dILCs. The abilities of antibodies to penetrate certain tissues and to deplete cells were assessed by treating mice intraperitoneally with $0.2 \mathrm{mg} \alpha \mathrm{CD} 4$ (GK1.5) or 1mg aCD90 $(30 \mathrm{H} 12)$ and then assessed the detectable $\mathrm{CD} 90^{+}$cell in the skin, auricular draining LN and spleen 48 hours later (Figure 5.8). There was marked decrease in the detectable $\mathrm{CD}^{+}{ }^{+}$cells in the spleen with $\alpha \mathrm{CD} 4$ treatment and a total loss from the spleen with $\alpha \mathrm{CD} 90$. In the LN there was no difference with GK1.5 treatment though $\alpha \mathrm{CD} 90$ caused an almost 10 fold decrease in the detectable CD90 ${ }^{+}$cells. In the skin no effect of the antibody treatments was observed, no masking of the CD90 staining or any apparent depletion. Although antibody could reach and deplete cells from the spleen, and to a lesser degree the LN, $1 \mathrm{mg}$ of $\alpha \mathrm{CD} 90$ was insufficient to affect the ear skin cells; the lack of masking indicates the antibodies were not even able to reach the cells in this tissue (Figure 5.8).

In summary dILCs were not detected responding in any significant way to any of our inflammatory models or application of recombinant cytokine. While they did report constitutive production of IL-13 no changes in this cytokine expression were measured so no obvious role for this IL-13 expression could be inferred. Furthermore, it was found that dILCs were resistant to antibody depletion compared to cells from the spleen of LN, this meant inflammatory responses in the absence of dILCs could not be accurately assessed. 
a Naive
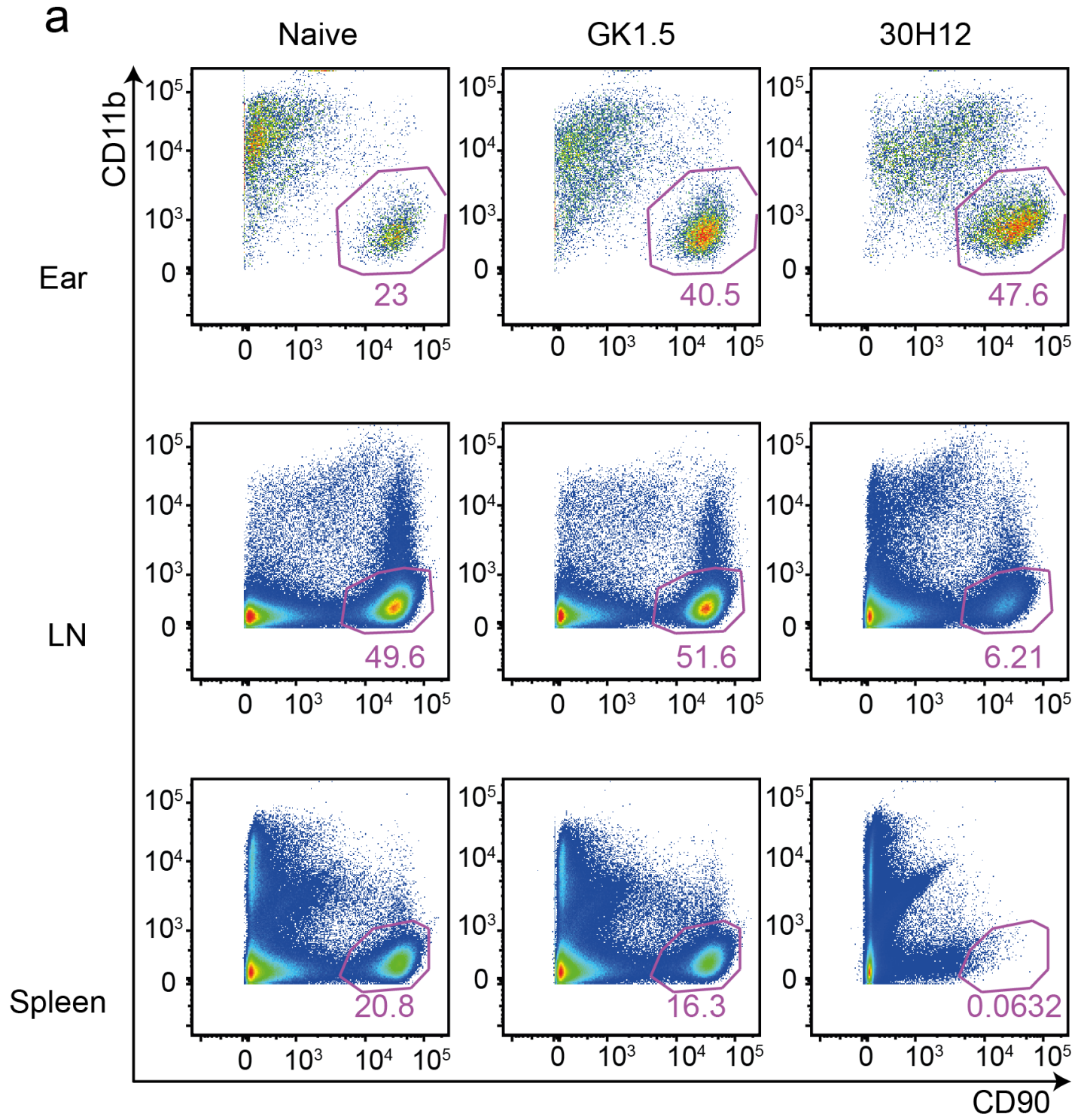

Figure 5.8 - ILGs of the skin are resistant to antibody depletion.

a. Representative flow plots of Live, $\mathrm{CD} 45^{+}$cells from the ear, auricular draining $\mathrm{LN}$ and spleens of mice 24 hours after receiving $0.2 \mathrm{mg}$ GK 1.5 (aCD4), $1 \mathrm{mg} 30 \mathrm{H} 12$ (aCD90) or remained naïve. Plots are representative of two independent experiments each $n=3$. 


\subsubsection{Basophils respond to TSLP induced by MC903 treatment by producing IL-4}

During the analysis of dILC responses I had observed an expansion of CD45 mid cells in the ears of MC903 treated mice (Figure 5.9a and b). These cells were found to be positive for CD49b (DX5), FceRI, and extracellular IgE their lack of expression of detectable C-kit confirmed their status as basophils (Figure 5.9a),. Recent publications had indicated that high levels of TSLP led to haematopoiesis of a population of basophils ${ }^{329}$. Knowing that the MC903 treatment caused increased serum levels of TSLP297 I hypothesised that TSLP signalling was causing increased systemic basophilia and migration into the skin. To test the systemic basophilia, basophil numbers and proportions of haematopoietic cells were analysed in the blood of mice treated on both ears with 4nmol MC903 or ethanol daily for 7 days. Unexpectedly the proportion of the CD45 expressing haematopoietic cells in the blood that were basophils decreased, this drop was also associated with a decrease in the number of basophils per $\mathrm{mL}$ of blood in the MC903 treated compared with ethanol treated mice (Figure 5.10a). I interpreted this drop in circulating numbers to indicate that the cells were likely migrating into tissues in response to treatment. To determine if the accumulation of basophils in the skin was treatment specific mice were treated for 7 days on one ear with $4 \mathrm{nmol} / \mathrm{ear}$ MC903 and ethanol on the other ear, and basophil numbers and their proportion of $\mathrm{CD}_{4} 5^{+}$cells in the ear skin (Figure 5.10b) and auricular draining LN (Figure 5.10c) were analysed. As observed previously, there were increased basophil numbers and proportions in the skin of MC903 treated ears and also increases in the LN from the treated side over the ethanol control ears or LN. The decreased levels of basophils in the blood of treated mice also correlated with infiltration of basophils into the skin and draining LN of mice but only specifically into the draining LN or the skin treated with MC903. 


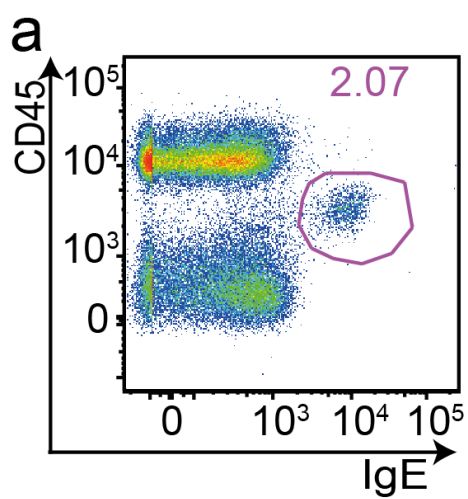

b

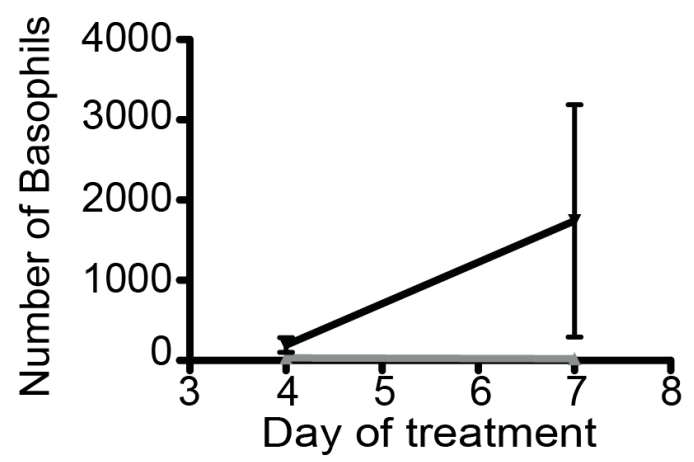

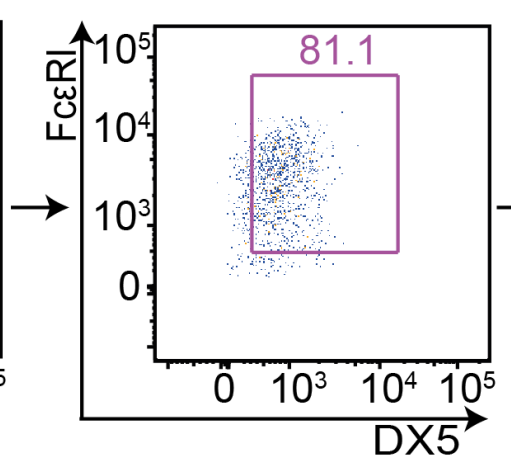

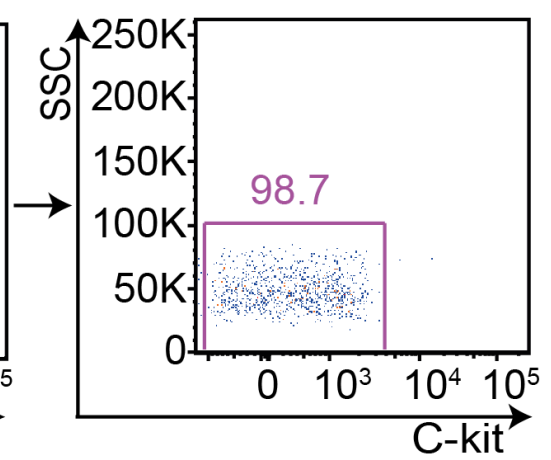

C
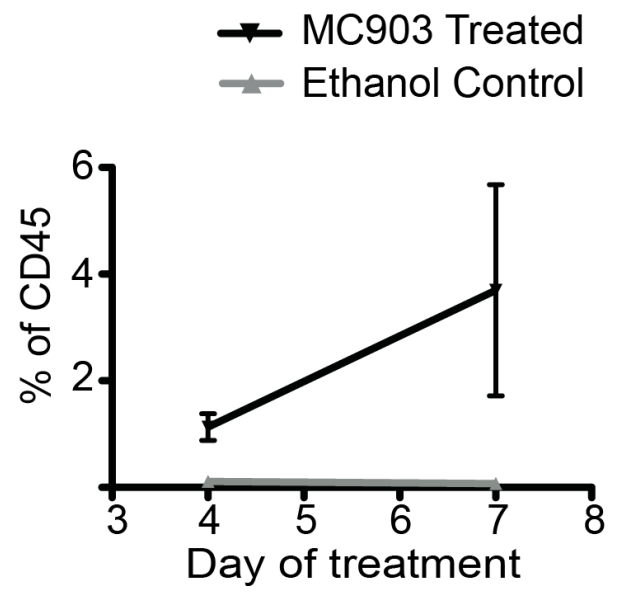

Figure 5.9 - Basophils migrate to the skin in response to MC903 treatment.

a. Representative plots of skin cells from ears treated for 7 days with MC903, pre-gated upon singlets then live cells. The first plot shows a CD45 ${ }^{\mathrm{mid}}$ population of cells staining positive for IgE; this population also stains positive for FceRI and DX5, are SSClo and is negative for C-kit. Gating is based upon FMO controls. b. Number, and c. proportion of $\mathrm{CD} 45^{+}$of basophils in ears of mice treated with $4 \mathrm{nmol} /$ ear/day of MC903 for 4 or 7 days. 
a

Basophils in blood
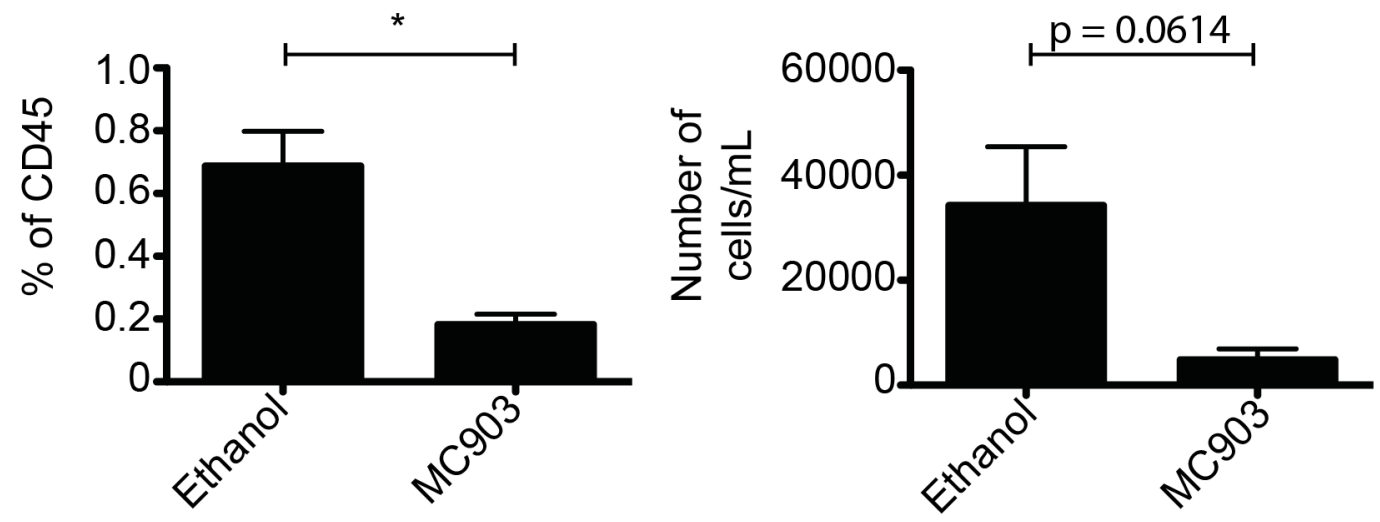

b
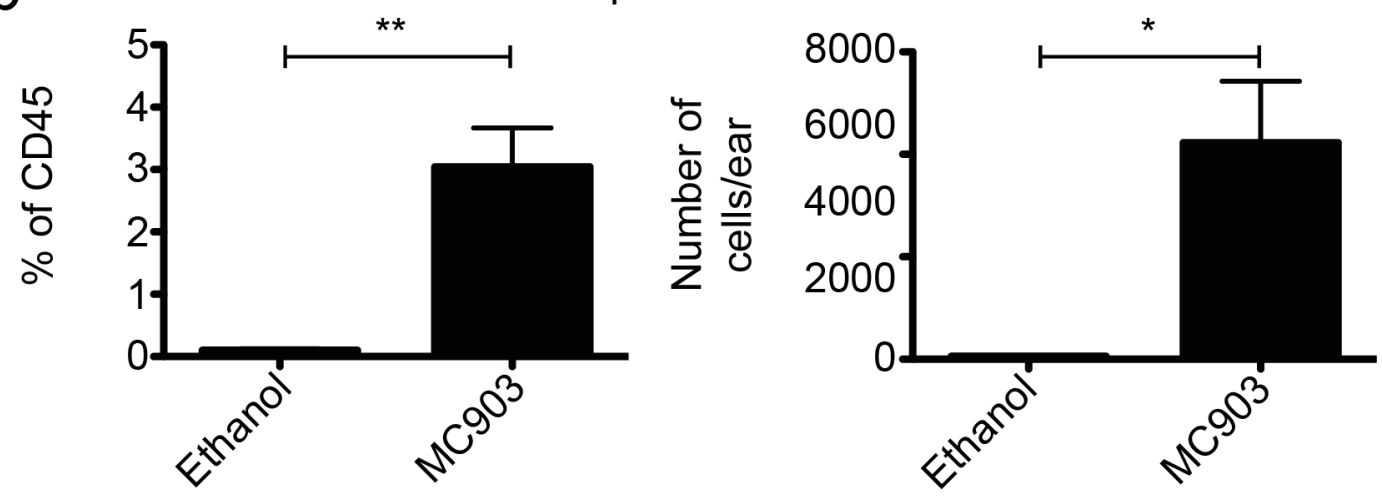

C
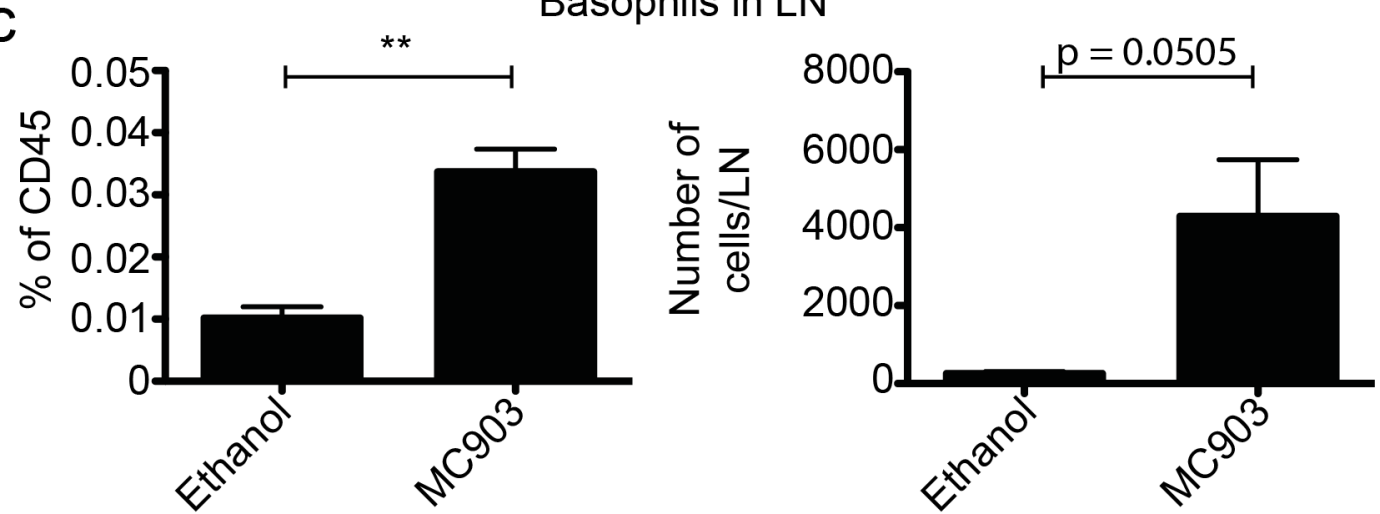

Figure 5.10 - Basophils migrate specifically to the site of treatment and the draining $L \mathbf{N}$.

Proportions of $\mathrm{CD}_{4} 5^{+}$and numbers of basophils in a. blood, b. ear and c. auricular draining LN on day 7 of MC903 treatment. a. Mice had been treated on both ears with either $4 \mathrm{~mol} / \mathrm{ear} /$ day of MC903 or ethanol. b. and c. mice received 4nmol/ear/day MC903 on one ear and ethanol on the other ear. All graphs show mean \pm S.E.M. for each group. Data are representative of two independent experiments. Statistics were calculated for each group using a Student's * $\mathrm{P}<0.05$ ** $\mathrm{P}<0.01$. 
One characteristic of G4 GFP IL-4 reporter mice is that basophils constitutively express GFP $^{330}$, so I decided to utilise this as an extra identifying marker of basophils in the various MC903 treated tissues. Using this technique an interesting phenomenon was noted in the treated mice. A population of basophils could be detected expressing higher GFP (GFPhi) than naïve basophils (Figure 5.11a). This was consistent with observations from our group whereby basophils in the lungs from $\mathcal{N}$. brasiliensis infected huCD2/GFP mice began expressing huCD2 and also increased their GFP MFI, to indicate they had begun expressing IL-4 ( 331 and unpublished data). Furthermore TSLP elicited basophils have been shown to produce very high levels of Type 2 associated cytokines particularly IL-4329, 332, so the proportions of basophils expressing high levels of GFP in the ear, LN and blood of GFP/IL-4 mice were analysed. A proportion of basophils in all three MC903 treated tissues began expressing GFP at a higher level than basophils in ethanol treated mice indicating they had begun expressing IL-4 (Figure 5.11b). IL-4 reporting was confirmed by looking at the reporter expression of basophils in the blood of 4C13R mice treated with MC903 for 7 days. A population of AmCyan expressing basophils could be detected reporting IL-4 expression. Interesting no DS-Red expressing basophils were detected indicating specific induction of IL-4 but not IL-13 expression (Figure 5.11c). 
a

Ear

LN

Blood
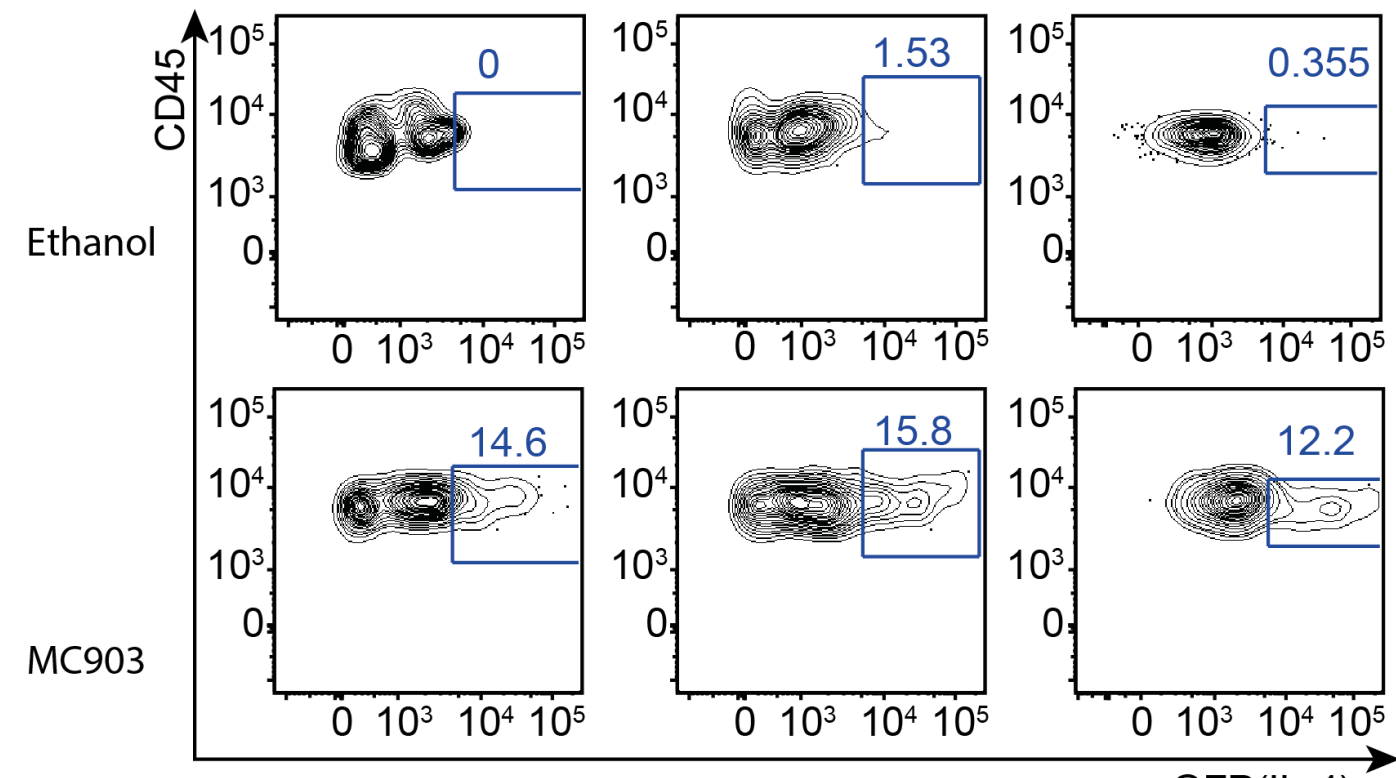

b
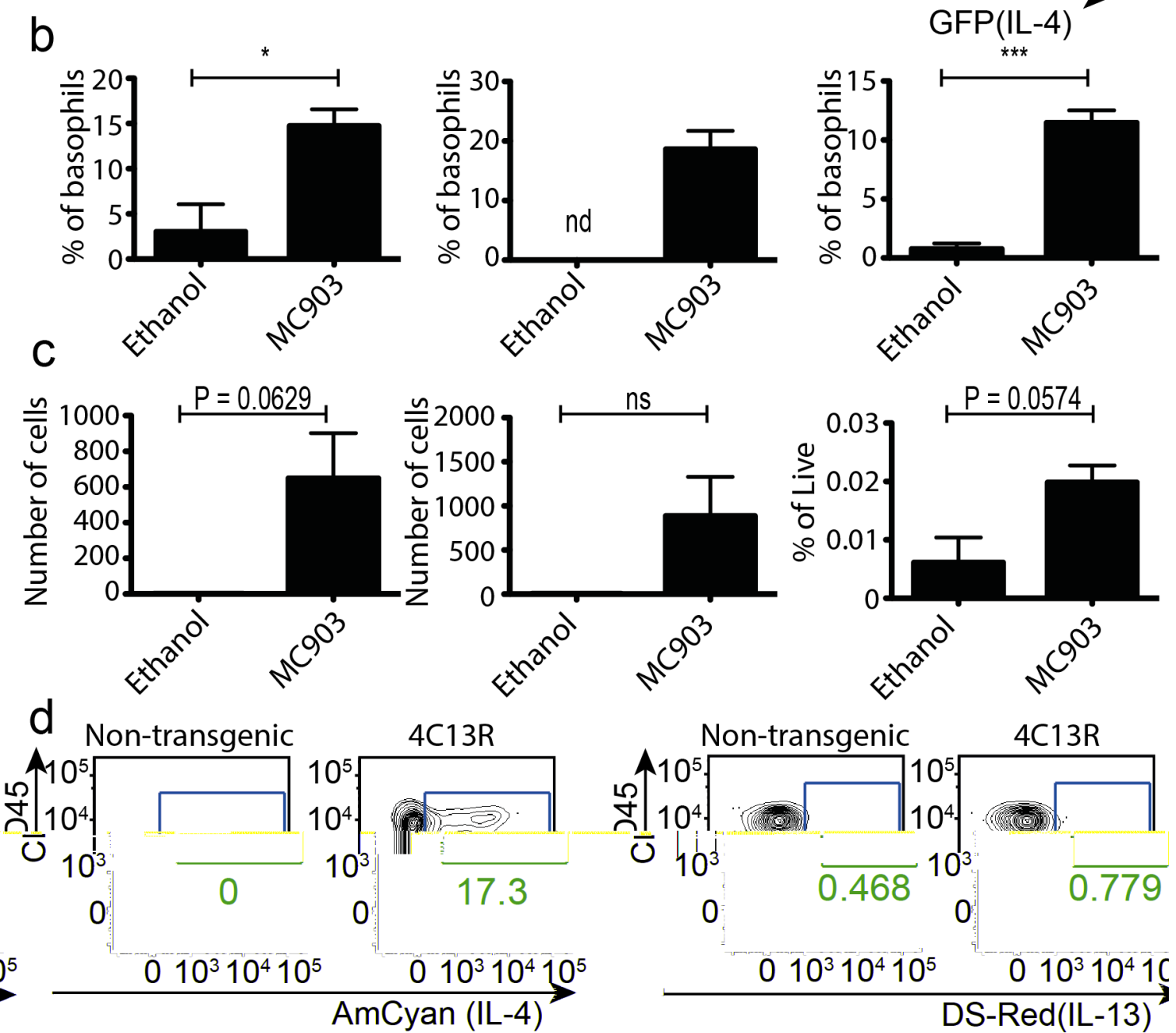

Figure 5.11 - MC903 elicited basophils produce IL-4 but not IL-13.

a. Representative plots, b. proportion of basophils and c. numbers of GFPhi basophils in ear, auricular draining LN and blood of mice receiving on both ears either 4nmol/ear/day MC903 or ethanol for 7 days. d. AmCyan and DS-Red expression of basophils in the blood of 4G13R and non-transgenic control mice after 7 days of MC903 treatment. All graphs show mean \pm 
S.E.M. for each group. Data are representative of two independent experiments. Statistics were calculated for each group using a Student's $* \mathrm{P}<0.05 * * * \mathrm{P}<0.001 \mathrm{~ns}=$ not significant.

TSLPR knock-out mice on a GFP IL-4 reporter background (GFP/GFP, so IL-4 deficient) were used to test my hypothesis that the basophilia, and increased IL-4 production by the basophils was dependent on TSLP. Comparing MC903 driven basophil responses in GFP/GFP mice with WT TSLPR (TSLPR ${ }^{+/}$) to TSLPR knockout mice $\left(\mathrm{TSLPR}^{-/}\right)$demonstrated tissue specific responses. Blood basophil numbers were higher in $\mathrm{TSLPR}^{-/}$mice, but they were decreased in the $\mathrm{LN}$ compared with TSLPR $^{+/+}$mice (Figure 5.12a). Unexpectedly there was no difference in number of basophils in the ear between the two strains of mice (Figure 5.12a). In all three tissues looked at a total lack of GFPhi induction by basophils from TSLPR ${ }^{-/-}$mice was observed (Figure 5.12b).

So I identified basophils as strong responders to MC903 exposure, although there was no measurable increase in basophil numbers in the blood, their migration into the ear skin and LN were measured in a treatment site-specific manner. MC903 treatment induced increased IL-4 reporting from basophils within all three tissues and this was dependent upon TSLPR signalling. 
a

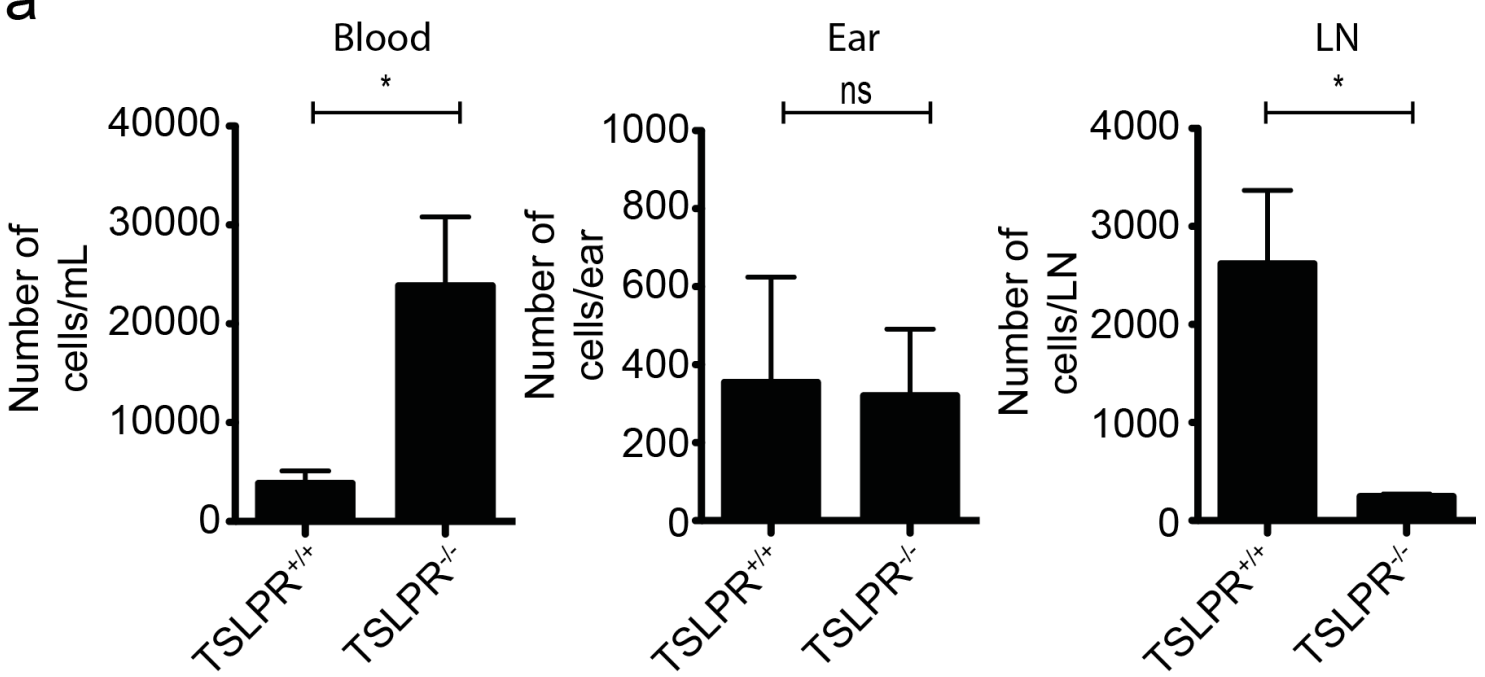

b
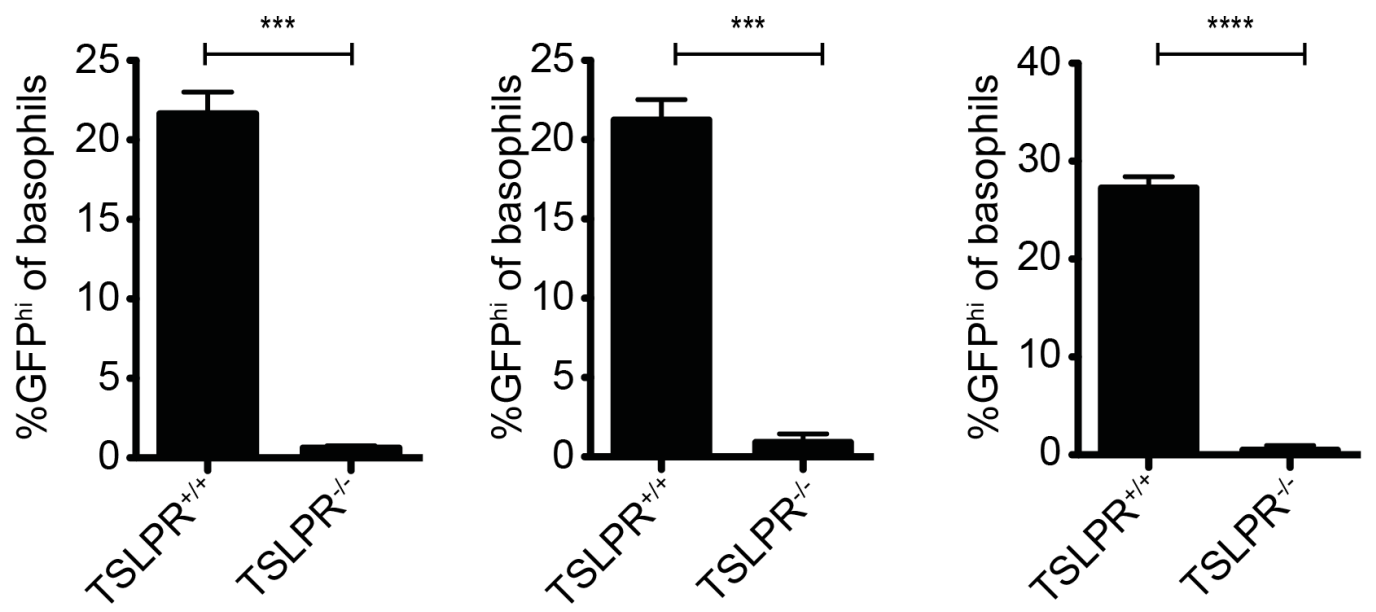

Figure 5.12 - TSLPR signalling is required for basophil IL-4 expression and migration to the $L N$.

a. Total numbers and b. proportion that are GFPhi of basophils in blood, ear skin and auricular draining LN of mice receiving on both ears either 4nmol/ear/day MC903 or ethanol for 7 days. All graphs show mean \pm S.E.M. for each group. Data are representative of two independent experiments. Statistics were calculated for each group using a Student's $* \mathrm{P}<0.05 * * \mathrm{P}<0.01$ $* * * \mathrm{P}<0.001 * * * * \mathrm{P}<0.0001 \mathrm{~ns}=$ not significant. 


\subsection{Discussion}

In this chapter I aimed to characterise a novel population of dermal resident innate lymphoid immune cells and observe their responses in a number of inflammatory conditions. I assessed the cytokine production of the dILCs and other immune cells found within inflamed skin using the IL-4 and IL-13 reporter mice. dILCs were found to have muted expansion and cytokine reporting responses but, treatment with MC903 induced cytokine production and migration of basophils into both the skin and the draining LN. How these cells contribute to pathogen specific immune responses has yet to be determined but these studies offer vital information about the immune cells of the skin and their cytokine responses.

The immune network in the skin is a carefully regulated system with cells inhabiting specialised sites in order to best carry out their functions. dILCs reside within the dermal layer of the skin, a position where they can have contact with DC subsets, macrophages, resident memory $\mathrm{T}$ cells and mast cells, but not with the dendritic epithelial T cells (DETCs) or Langerhans cells in the epidermis. ${ }^{333}$ Imaging studies performed by Roediger et al. utilising multiphoton imaging found that the dILCs were the only cells in the skin of Rag1/- mice that expressed CXCR6. Utilising Rag1/xCXCR6-GFP reporter mice two patterns of dILC movement were observed; active migration through the dermis and extended periods of remaining static. Through the use of bone marrow chimeras and other reporter mice they were able to show that the dILGs did not interact with DGs but did migrate towards mast cells, and halt their movement when in the close vicinity of a mast cell. ${ }^{265}$ These observations led to the hypothesis that dILCs might have a role in regulating dermal mast cell responses.

IL-13 is one of the key cytokines produced by the group 2 ILCs; the IL-13 reporting by dILCs indicates that at least a proportion of the population are ILC2s (Figure 5.3). The fact that the proportion of dILCs expressing IL-13 in naïve skin was inversely proportional with age could indicate several things. It could indicate the signals that drive IL-13 expression by dILGs dissipates as the animal ages, or that there is a preferential outgrowth of other ILC populations that begin to outnumber the ILC2s. IL-13 expression in the skin is usually associated with fibrosis and inflammation as seen in systemic sclerosis ${ }^{334}$ and models of atopic dermatitis ${ }^{205}$, however its roles in naïve skin were unknown. Following the discovery that these cells report IL-13 and also interacted with mast cells the cytokine responses of mast cell cultures activated by receptor bound 
IgE cross-linking in the presence of IL-13 were tested. IL-13 was found to inhibit mast cell cytokine release, potentially hinting at a role of dILCs as regulatory cells of the skin controlling IgE driven mast cell responses. ${ }^{265}$ This proposition potentially fits with the observation that atopy is most prevalent in young children ${ }^{335}$. The higher proportions of IL-13 expressing dILCs in younger animals could be acting to inhibit over-activation of mast cells in the skin in situations of high levels of circulating $\operatorname{IgE}$ in early life. The lack of IL-4 expression by dILCs is consistent with other reporters assessing cytokine expression by unstimulated ILCs directly ex vivo 257 .

This study observed minimal expansion and changes in IL-13 reporting by the dILCs in a range of inflammatory conditions, however Roediger et al. found that dILCs would respond to IL-2. During experiments where mice were treated with IL-2 complexed with antibody (IL-2c) in an attempt to maintain adoptively transferred Tregs, it was noted that the dILGs expanded greatly. IL2c treatment of Rag 1/- mice induced strong dILC expansion associated with increases in ILC2 cytokines IL-5 and IL-13. These mice also spontaneously developed skin lesions populated by neutrophils, eosinophils and dILCs that were characteristically similar to lesions seen in atopic dermatitis patients. ${ }^{265}$ It is possible that in this study strong dILC responses were not registered as these experiments assessed early time points looking for direct dILC activation by damage or invading pathogens, while dILC activation may actually occur subsequent to the activation of the adaptive immune system. If dILCs do rely upon the IL-2 provided by $\mathrm{T}$ cells migrating to the site of inflammation then they could either act as supporting cells by producing extra cytokine to sustain inflammatory responses as may be the case in atopic dermatitis, or they could play a regulatory role. The production of cytokines such as IL-13 could counter the effects of other inflammatory cytokines such as IFN- $\gamma^{336}$ and IL-1 7337 , while promoting healing and fibrosis ${ }^{336}$.

I measured only marginal dILC responses in the skin of MC903 treated mice (Figure 5.6), however papers published since this work was carried out have measured TSLP dependent ILC2 changes within the skin and draining LN 338,339 . Furthermore it was found that antibody treatment was capable of depleting ILGs from the LN and this antibody depletion could diminish the MC903 driven ear thickening. Whether this study actually achieved depletion of ILC2s within the skin was not demonstrated, so either depletion was achieved and it was the absence of skin resident ILC2s that explained the decreased inflammation, or that the antibody sensitive LN ILC2s were critical to driving the MC903 inflammation. In either case there was significant enough 
differences between this study and the one by Kim et al. to leave several questions unanswered in relation to the role of ILCs in MC903 inflammation.

The MC903 model did highlight strong migratory responses, and IL-4 but not IL-13 expression by basophils to the vitamin D3 analogue (Figure 5.9). IL-4 producing basophils in the skin have been associated with the alternate activation of skin macrophages, which can trap parasites as they migrate through the $\operatorname{skin}^{340}$. Siracusa et al. published a paper identifying a novel subset of basophils that respond to TSLP. These basophils expressed high levels of Type 2 cytokines and were quite different to traditional IL-3 elicited basophils, particularly in the repertoire of proteases they expressed and a notable lack of histamine. This paper also used the MC903 model and discovered TSLP dependent, and IL-3 independent, basophilia in the ear. In addition to my discovery that these basophils were also present, albeit in low number, and reporting high IL-4 expression in the draining LN of treated ears, Siracusa et al. found that basophil depletion decreased the amount of Type 2 cytokine produced by restimulated $\mathrm{T}$ cells from MC903 treated draining LNs. These data indicated that basophils might play a role in the generation or expansion of Th2 responses, in the draining LN. 329 A subsequent paper published after the conclusion of this project evaluated the importance of TSLP elicited basophils in the induction of Th2 in the mesenteric LN during intestinal helminth infection and found these basophils required for optimal Th2 generation ${ }^{341}$.

Conflicting reports exist proposing basophils are or are not required for the generation of Th2 cells in vivo. Th2 differentiation requires IL-4 in vitro however it was long thought Th2 cells were the only source for IL-4 in the LN so a chicken/egg causality dilemma existed as no obvious source of IL-4 was known that could switch activated CD4 ${ }^{+} \mathrm{T}$ cells to Th2 $2^{342}$. The discovery of basophils within the reactive $\mathrm{LN}$ and also that basophils could express MHC II and co-stimulatory molecules made them an attractive candidate as cells that could present antigen and produce the IL-4 that would induce Th2 differentiation ${ }^{343}, 344,345,346$. Several studies claimed that basophils were more important than DCs at inducing Th2 cells, anti-FceRI antibody targeting of basophils ${ }^{344,}$ 345 and limiting of MHC II expression to $\mathrm{CD} 1 \mathrm{lc}^{+}$cells $^{346}$ greatly diminished Th2 generation. These papers were challenged however by the implementation of CD11cdiptheria toxin receptor (DTR) depletion models, where by loss of CD11c expressing cells significantly inhibited Th2 $2^{347}$. The further identification of FceRI expressing inflammatory DGs which were both necessary and required for in vivo Th2 induction 
brought into question the purity of the previously identified basophils that could present antigen, and provided an alternate explanation as to why FceRI targeting antibody disrupted Th2 generation ${ }^{348}$. Despite these controversies recent developments of genetic based basophil depletion models have generated further contradictory data, which in some models indicate clearly no role for basophils, ${ }^{349}$ and others claiming basophils are required for optimum responses ${ }^{341,350}$. While enough evidence exists that basophils can affect Th2 responses it remains uncertain if they truly do in vivo and if they do to what extent. Careful analysis using well characterised models and specific depletion models will educate $\mathrm{Th} 2$ researchers in the future.

\subsection{Conclusions}

This study acts as a foundation for future research into the roles of innate immune cells in the skin, particularly in relation to Type 2 immune responses. I characterised a novel cell type, the dILC and detected significant, age dependent, IL-13, but not IL-4 reporting by these cells. While these cells appeared unreactive in the inflammatory models assessed, more in depth time course studies may discover as yet unrealised roles for these cells, particularly in light of the data that they strongly respond to IL-2 which can be produced by T cells. Basophils display a TSLP dependent cytokine response in a model of skin inflammation caused by treatment with an analogue of vitamin D3. These basophils tracked specifically to the treated area of the mouse and could also be detected within the LN; they produced IL-4 in a TSLP dependent manner but were not observed to be producing IL-13. Publications by other groups indicate these basophils may have a role in the induction of Th2 cell responses within the LN and further studies could determine if there is a link between epithelial cell derived TSLP, IL-4 producing basophils and the generation of atopic dermatitis. These data also demonstrate a further differentiation in Type 2 cytokine expression, with two distinct populations of cells each expressing IL-4 or IL-13 within the same tissue. While CD4+ ${ }^{+}$cells may differentially regulate their timing and location of expression, some cell types can selectively express only one of these cytokines. 


\section{Interplay of adaptive and innate immune responses mediate protection against Nippostrongylus brasiliensis}

\subsection{Introduction}

Upon secondary exposure to the rodent hookworm $\mathcal{N}$. brasiliensis WT mice mount protective immune responses, the nature of which have been widely characterised ${ }^{127,}, 132$, 134, 340, 351 but the exact mechanisms contributing to protection remain ambiguous. While much research has focused on the intestinal expulsion phase seen in late primary infection $^{14,73,254,352,353}$, we have focused on the phenomena of protection, whereby worms are killed within the host by the immune responses, not simply expelled. As fewer worms arrive in the intestine in a secondary infection they must be trapped or killed in another site earlier during their transit of the host ${ }^{132}$. Traditionally infectious larvae are injected subcutaneously for experimental infections ${ }^{124}$. Using a technique comparing subcutaneous versus intravenous injection of larvae, other researchers in this lab established a model for identifying the location of the protective responses ${ }^{354}$. Larvae injected intravenously arrive in the lung within minutes and 2 days after infection, mice 
that have previously received a primary infection have fewer surviving larvae in the lung than mice being infected for the first time. Comparing the number of surviving larvae in primed mice 2 days after infection either intravenously (where larvae can only be killed in the lung) or subcutaneously (where larvae can be killed in the skin while migrating to the lung as well) demonstrates that between $60-80 \%$ of protective responses occur in the lung ${ }^{354}$. Our lab has previously found the lungs to be a critical site for priming protective immune responses ${ }^{354}$, so in this chapter we have focused on immune mechanisms within the lung that mediate larval killing.

Th2 cells are critical to protective responses, as are the cytokines that signal through STAT6 ${ }^{132}$. Measurement of Type 2 cytokine expression has required the use of in vitro restimulation ${ }^{355}$ or knock-in reporter mice ${ }^{31,311}$, the following data utilises the 4C13R IL-4 and IL-13 transgenic reporter system to identify and characterise the cytokine producing cells in the lung during infection in an immune competent environment. Using this reporter mouse, I have characterised lung ILC2 activation and cytokine contributions during primary and secondary infections. ILC2s have roles in expulsion of $\mathcal{N}$. brasiliensis from the gut ${ }^{254}$ and also in mediating asthmatic responses ${ }^{257}$ and healing following viral infection in the lungs ${ }^{240}$, but prior to this study a direct role for ILC2s in protective responses against larvae in the lung has not been established. ILC2s act as sensors of barrier damage, responding to cytokines including TSLP 338 and IL-33253 released by injured epithelial cells ${ }^{356}$. IL-33 has also been shown to have a role in protective responses against $\mathcal{N}$. brasiliensis with IL-33\%-- mice having decreased protection against a secondary infection leading to greater numbers of larvae reaching the intestine $^{353}$. This chapter assesses the roles of ILC2s in secondary infection and also in naïve mice treated with ILC2 stimulatory cytokines prior to infection.

ILC2s have also been shown to interact with $\mathrm{CD}^{+} \mathrm{T}$ cells in several different ways. Both ILC2254 and ILC3357 populations express low levels of MHC II. Antigen dependent interactions between ILC3s and $\mathrm{CD} 4^{+} \mathrm{T}$ cell have been demonstrated to suppress CD4 ${ }^{+} \mathrm{T}$ cell responses due to the lack of co-stimulatory factors on the ILC3 $\mathrm{s}^{357}$. ILC2s have been shown to support the generation of Th2 responses in response to $\mathcal{N}$. brasiliensis infection and Th2 differentiation and proliferation in vitro ${ }^{358}$. ILC2s strongly respond to IL-2 265 , which largely derives from activated $\mathrm{T}$ cells. In this study we have assessed the interactions between $\mathrm{CD} 4{ }^{+} \mathrm{T}$ cells and ILC2 responses in the lung during $\mathcal{N}$. brasiliensis infection. 


\subsubsection{Aims}

I investigated how different immune cell populations contribute to trapping and killing of the $\mathcal{N}$. brasiliensis larvae during the lung infectious phase. The responses of $\mathrm{CD}^{+}{ }^{+} \mathrm{T}$ cells and ILC2s were analysed and the roles of Type 2 cytokines in activating lung macrophages, which are critically required for protection against the parasite, assessed. Specifically these experiments aimed to:

- Identify innate lymphoid cells of the lung and characterise the IL-13 producing cells during a secondary infection.

- Compare IL-13 production by ILC2s and $\mathrm{CD}^{+} \mathrm{T}$ cells and compare the contribution to protection by IL-4 and IL-13.

- Measure $\mathrm{CD}^{+} \mathrm{T}$ cell roles in protection against reinfection and interactions with ILC2s

- Test the ability of treatment with different cytokines to induce protective immune responses without priming or in secondary infection in the absence of CD4 ${ }^{+} \mathrm{T}$ cells.

\subsection{Results}

\subsubsection{ILC2s contribute to IL-13 production in the lung during $\mathbf{N}$. brasiliensis infection}

4C13R reporter mice were infected via i.v. injection with $\mathcal{N}$. brasiliensis to assess cytokine expression by immune cells within the lung. As expected from previous reports, CD4 ${ }^{+}$ $\mathrm{T}$ cells were significant producers of IL-13 but a population of CD3- CD4- cells expressing the IL-13 reporter DS-Red could also detected (Figure 6.1a). Based upon observations with skin resident dILGs expressing IL-13, I designed a staining panel to allow identification of ILC2s in the lung and assess their contribution to cytokine production (Figure 6.1b). By gating on CD45+ CD11b- CD3- NK1.1- B220- CD2+ $\mathrm{CD}^{+} \mathrm{CD}^{+} 5^{+}$a distinct population in the lung was identified. The majority of these cells express the ILC2 associated transcription factor GATA3 and the IL-33 receptor T1-ST2. Furthermore a small proportion of these cells expressed DS-Red, reporting IL13 production in the lung of naïve $4 \mathrm{C} 13 \mathrm{R}$ mice similar, though to a lesser extent, to what had been seen from dILCs in the skin (Figure 6.1b). 
a
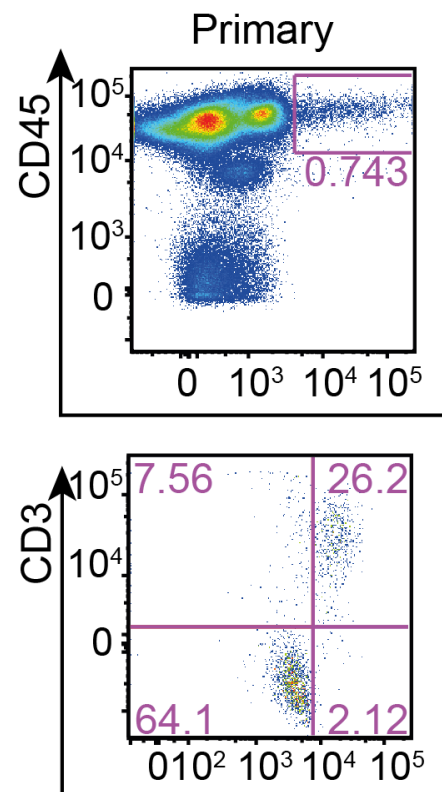
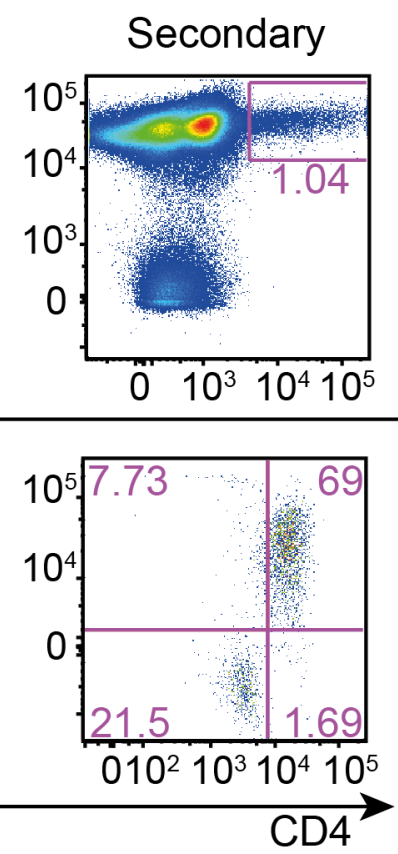

Non-transgenic

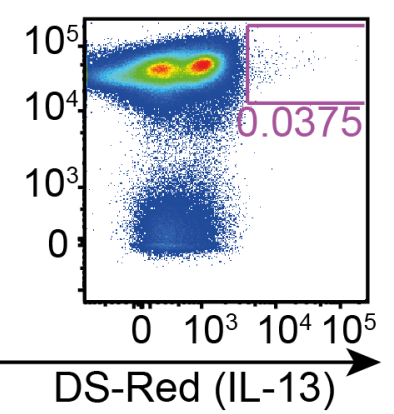

\section{b}
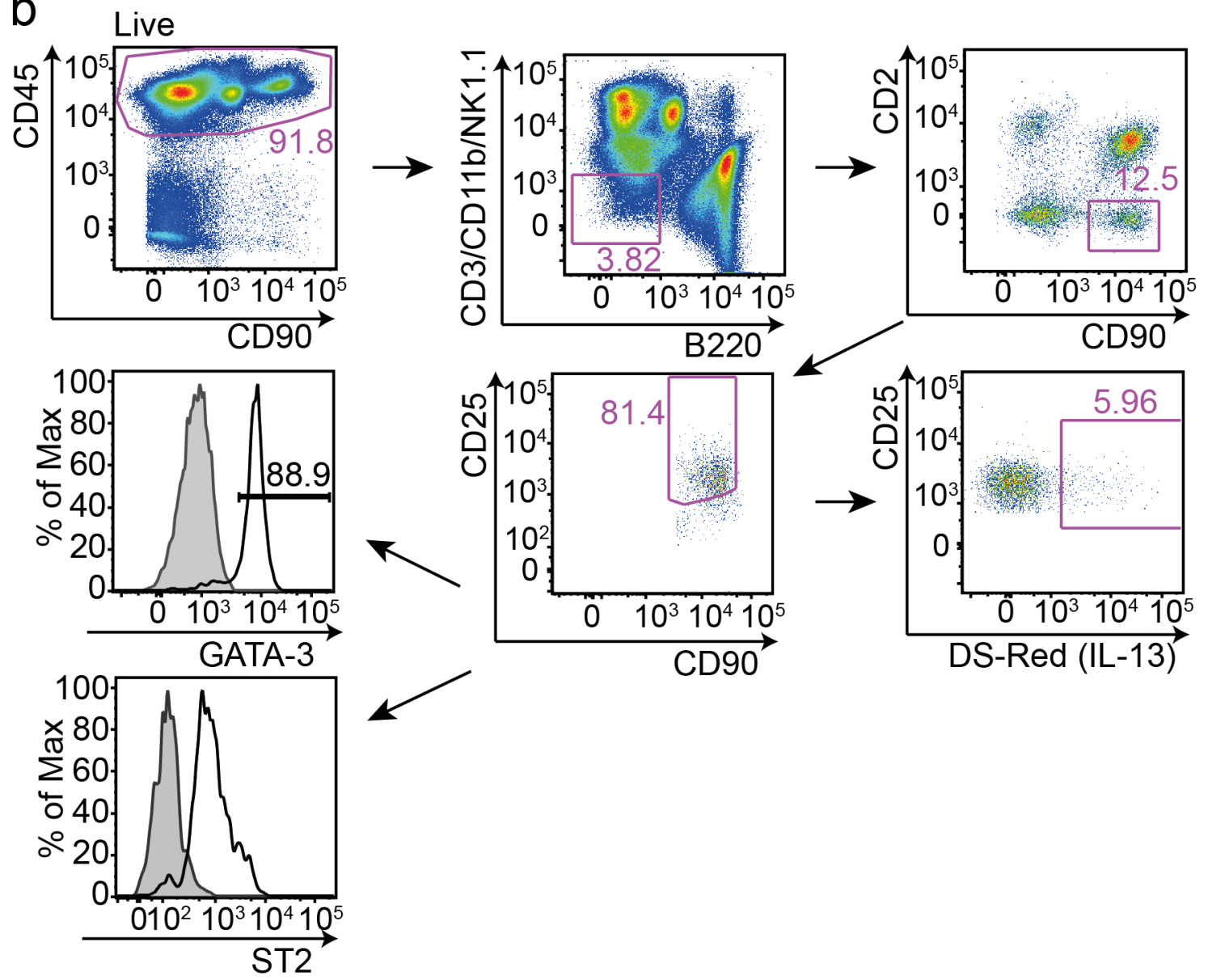

Figure 6.1 $-N$. brasiliensis responsive DS-Red ${ }^{+}$non-GD4 lymphoid cells in the lung are ILC2s.

a. Identification of CD3-CD4- DS-Red expressing cells from the lung in primary and secondary $\mathcal{N}$. brasiliensis infection. DS-Red gating based on WT cells. Plots are representative of multiple experiments. b. Gating scheme for identifying innate lymphoid cells from the lung, cells are 
CD45+, CD3-. CD11b-, NK1.1-, B220-, CD25+, CD90+. Expression of GATA3, ST2 and DSRed by ILC2s from naïve mice is shown.

STAT6 signalling is known to be important for providing protection during reinfection ${ }^{132}$, however the contribution of IL-13 to the protective immune responses has not been assessed. To begin I identified the major IL-13 producing populations in the lungs during the secondary infection. Cells were isolated from the digested lungs of 4G13R mice 2 days after their secondary infection, and panels were designed specifically to minimise the background fluorescence in the DS-Red channel, allowing sensitive identification of basophils, ILC2s, CD4 and CD8 T cells, neutrophils and eosinophils by flow cytometry. Live cells were selected for and then gates encompassing all lung cells expressing DS-Red with a higher fluorescence than the background of an infected non-transgenic mouse were set (Figure 6.2a). Then the cell populations that made up the reporter positive population were determined. Approximately $40 \%$ of the IL-13 reporting cells were CD4 ${ }^{+} \mathrm{T}$ cells, 15\% basophils and 25\% ILC2s (Figure 6.2b). Via this gating method about $20 \%$ of the DS-Red positive cells were unaccounted for. This process was repeated for IL-4 reporter positive cells and I found that basophils made up the largest population of IL-4 expressing cells $(\sim 32 \%)$, followed by CD4 ${ }^{+} \mathrm{T}$ cells $(\sim 21 \%)$ and eosinophils $(\sim 9 \%)$ (Figure $6.2 \mathrm{~b})$. This was inconsistent with previous reports that at day 6 of infection, greater numbers of CD4 $4^{+} \mathrm{T}$ cells were producing IL-4 than basophils in the secondary responses ${ }^{331}$, however the differences may be due to the earlier time point of day 2 post infection assessed here. Some of the ILC2s expressed IL4 , (3\% of the ILC2 population) although a small proportion, this did indicate the potential of ILC2s to make IL-4 under some conditions (Figure 6.2b).

Concomitant experiments performed in our laboratory by Dr. Tiffany Bouchery addressed the role of lung macrophages in protecting against secondary infection. It was found that lung macrophages take on an alternately activated (M2) phenotype in a secondary infection, characterised by expression of Arginase and resistin-like molecule alpha (Relm- $\alpha$ ) and the phosphorylation of STAT6 ${ }^{354}$. To assess the sufficiency of M2 macrophages to provide protection, bone marrow derived macrophages were cultured and pulsed overnight with IL-4 to drive an M2 phenotype. These cells were adoptively transferred intranasally delivering them to the lungs of naïve mice; these mice were then challenged with $\mathcal{N}$. brasiliensis and the numbers of larvae in the lung were enumerated 2 days post infection. It was found that adoptive transfer of M2 macrophages was enough 
to induce protection, though transfer of LPS primed macrophages did not affect lung larvae numbers ${ }^{354}$. 
a
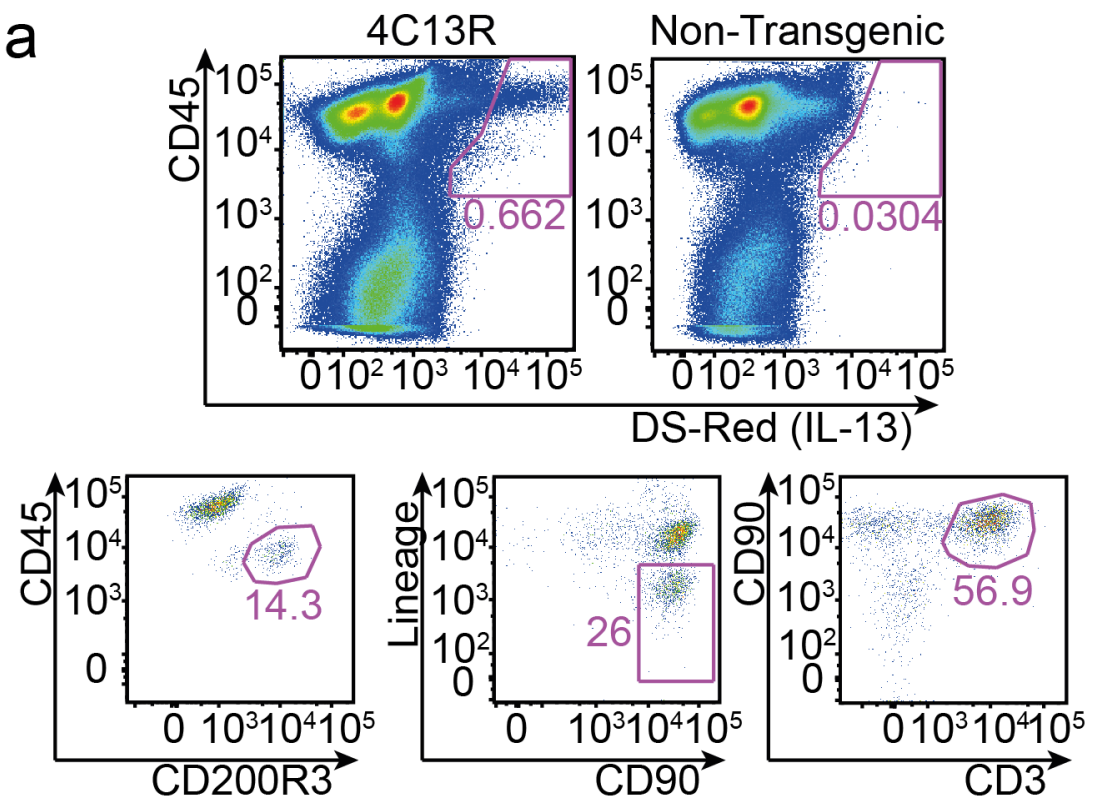

b
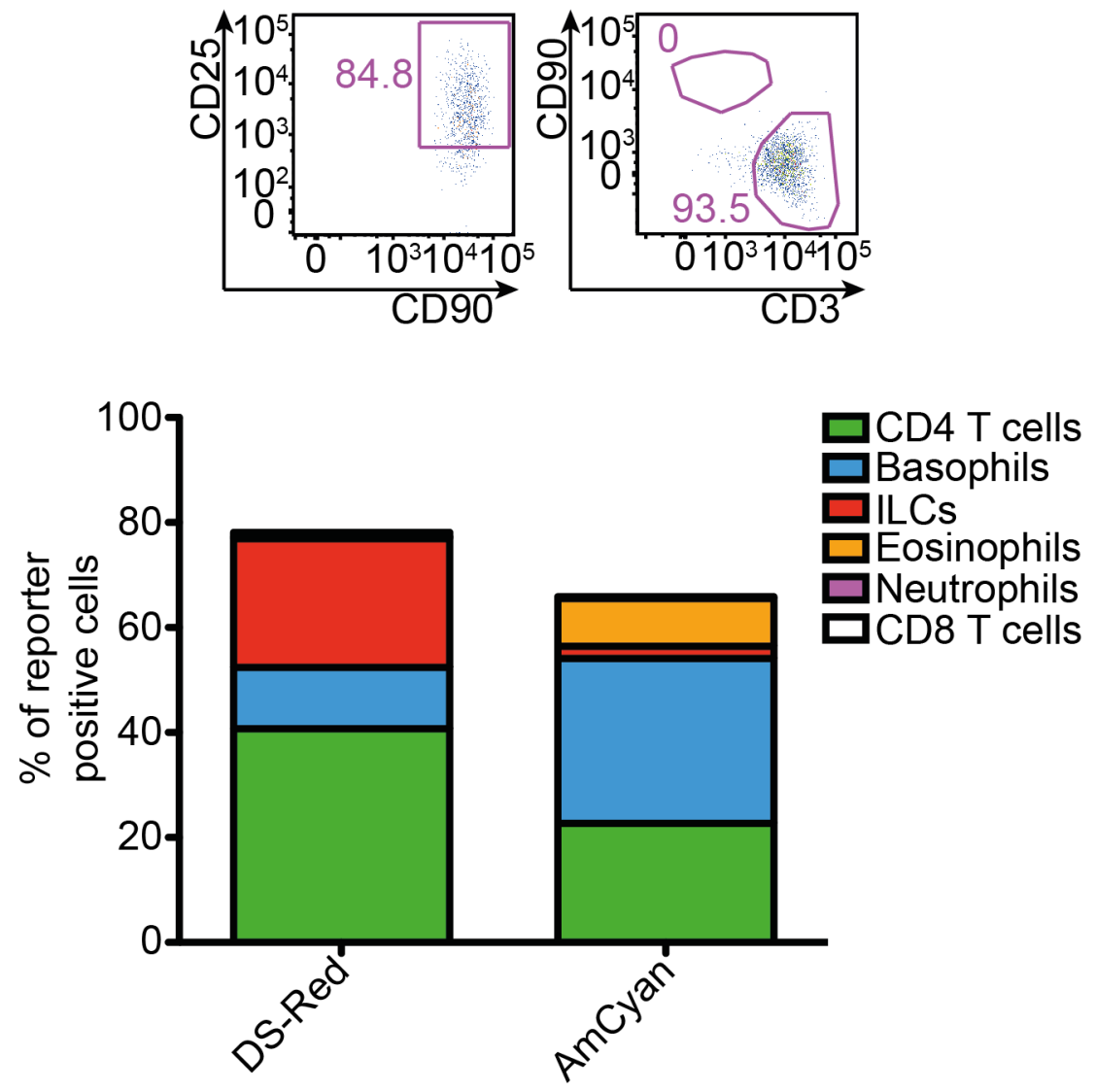

Figure 6.2 - GD4+ $T$ cells and ILG2s are the majority IL-13 producing cells during secondary infection.

a. 4C13R mice were infected via i.v. with $\mathcal{N}$. brasiliensis, and then reinfected 30 days later. 2 days after secondary infection lungs were harvested and digested and stained with a range of panels to identify immune cells producing IL-13 as reported by DS-Red. Total live cells were identified then all the DS-Red ${ }^{+}$cells were gated on, gates based upon infected non-transgenic lung cells. From the DS-Red ${ }^{+}$population; CD45mid CD200R3+, identified basophils, ILC2s by lineage$\mathrm{CD}^{+}{ }^{+} \mathrm{CD}_{25}{ }^{+}$, $\mathrm{T}$ cells as $\mathrm{CD} 90^{+} \mathrm{CD}^{+}$and $\mathrm{CD} 4^{+}$or $\mathrm{CD}^{+} \cdot \mathbf{b}$. The composition of DS-Red ${ }^{+}$ and AmCyan ${ }^{+}$cells in the lungs of secondary infected mice. Cells were gated as per $\mathbf{a}$. and then the proportion that each cell type contributed to the reporter positive population was calculated. 
Eosinophils were defined as Siglec F+ GD11c- SSChi and neutrophils as Ly6Chi Grlhi. Bar graph shows a representative sample. Data is representative of greater than five independent experiments.

We hypothesised that both IL-4 and IL-13 in the lungs are able to induce M2 activation of macrophages by signalling through STAT6 and these macrophages were providing protection through either direct or indirect mechanisms. To test the relative contribution of IL-4 and IL-13 an IL-13R blocking antibody was generously provided by AbbVie and used to treat WT and IL-4 deficient (GFP/GFP) mice a week before, and during the secondary infection. WT and IL-4 deficient mice had equal numbers of larvae at 2 days post primary infection, and there was no significant difference between the WT and IL-4 deficient mice 2 days post secondary infection (Figure 6.3a). Although not significant, there was a slight trend towards reduced protection in the IL-4 deficient mice. $\alpha$ IL-13R antibody treatment also made no difference to the larval number in secondary infected WT mice. Treatment of IL-4 deficient mice with $\alpha$ IL-13R antibody totally abrogated protection with these mice having the same number of larvae in the lung as primary infected mice.

Through these experiments we have discovered that the STAT6 dependent protection against $\mathcal{N}$. brasiliensis infection in the lung has redundancy. Single blockade of IL-4 or IL-13 signalling does not affect protection, however simultaneous blocking of both IL-4 and IL-13 signalling led to total loss of protective mechanisms. 
a

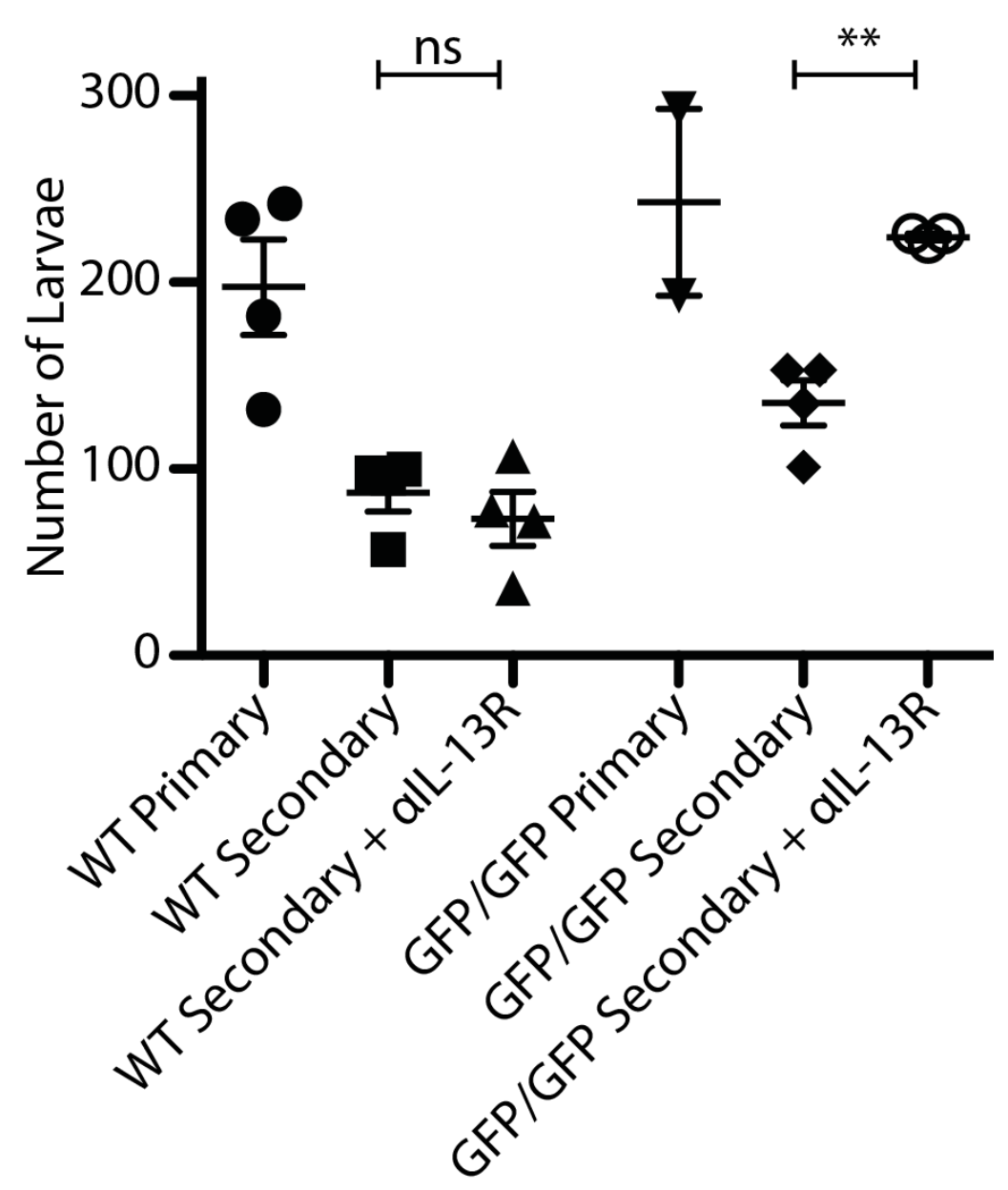

Figure 6.3 - Requirement for IL-4 or IL-13 signalling to maintain protection.

a. Numbers of larvae recovered from digested whole lungs from wild type and IL-4 deficient mice 2 days after either primary infection or secondary infection with or without treatment with aIL-13 antibody the week preceding secondary challenge. Graph shows mean \pm S.E.M. in each group. Data are from a single experiment. Statistics were calculated for each group using One way ANOVA with Bonferroni multiple comparisons post test $* * \mathrm{P}<0.01 \mathrm{~ns}=$ not significant. 


\subsection{2 $\mathrm{CD4}^{+} \mathrm{T}$ cells support ILC2 responses in secondary $\mathbf{N}$. brasiliensis infection}

The IL-4 producing cells in secondary infected lungs have been identified and their

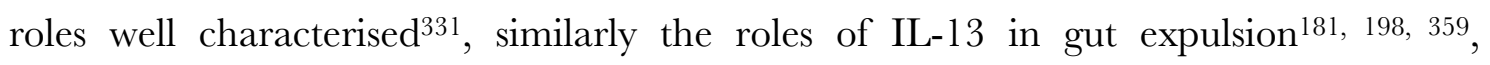
however the contributions of IL-13 to protective responses in the lung has not been well studied. With recent publications indicating that ILC2s and CD4+ $\mathrm{T}$ cells interact, and that ILC2s may be critical for Th2 cell differentiation and activation ${ }^{358}$, I wished to measure the kinetics of the responses by these cell populations and their potential interactions.

Initially the early responses of $\mathrm{CD} 4^{+} \mathrm{T}$ cell and ILC2s were assessed, the kinetics of cellular activation were measured during a time course between 24 hours and 48 hours post primary infection. The numbers of each of these cell types did not alter dramatically over the time course (Figure 6.4a). The proportions (Figure 6.4b) and numbers (Figure 6.4c) of DS-Red expressing ILC2s were consistently greater than IL-13 reporter positive $\mathrm{CD} 4{ }^{+} \mathrm{T}$ cells indicating that ILC2s were activated earlier than $\mathrm{T}$ cells in response to $\mathcal{N}$. brasiliensis infection.

As greater numbers of DS-Red ${ }^{+}$ILC2s than IL-13 reporting CD4 ${ }^{+} \mathrm{T}$ cells were measured at 2 days after primary infection I compared their numbers 2 days after secondary infection and 30 days after a primary infection (primed). Very few DS-Red ${ }^{+}$ ILC2s or $\mathrm{CD}^{+} \mathrm{T}$ cells were found in the primed lungs, with a significant decrease in the number of IL-13 reporting ILC2s from 2 days post primary (Figure 6.4d). The lack of difference between primary and primed DS-Red expressing CD4 ${ }^{+} \mathrm{T}$ cells is likely explained by their cytokine response beginning and resolving between day 2 and day 30 . In secondary infection both ILC2 and CD4+ T cell DS-Red responses were expanded, similar numbers of DS-Red ${ }^{+}$cells from each of these populations were enumerated in the lungs. So based on these results, ILC2s respond faster than CD4 ${ }^{+} \mathrm{T}$ cells in the primary infection, the IL-13 reporting by both populations resolves by day 30 post primary infection but 2 days after secondary infection they respond quickly with equal numbers of DS-Red ${ }^{+}$ILC2s and CD4 ${ }^{+} \mathrm{T}$ cells. 

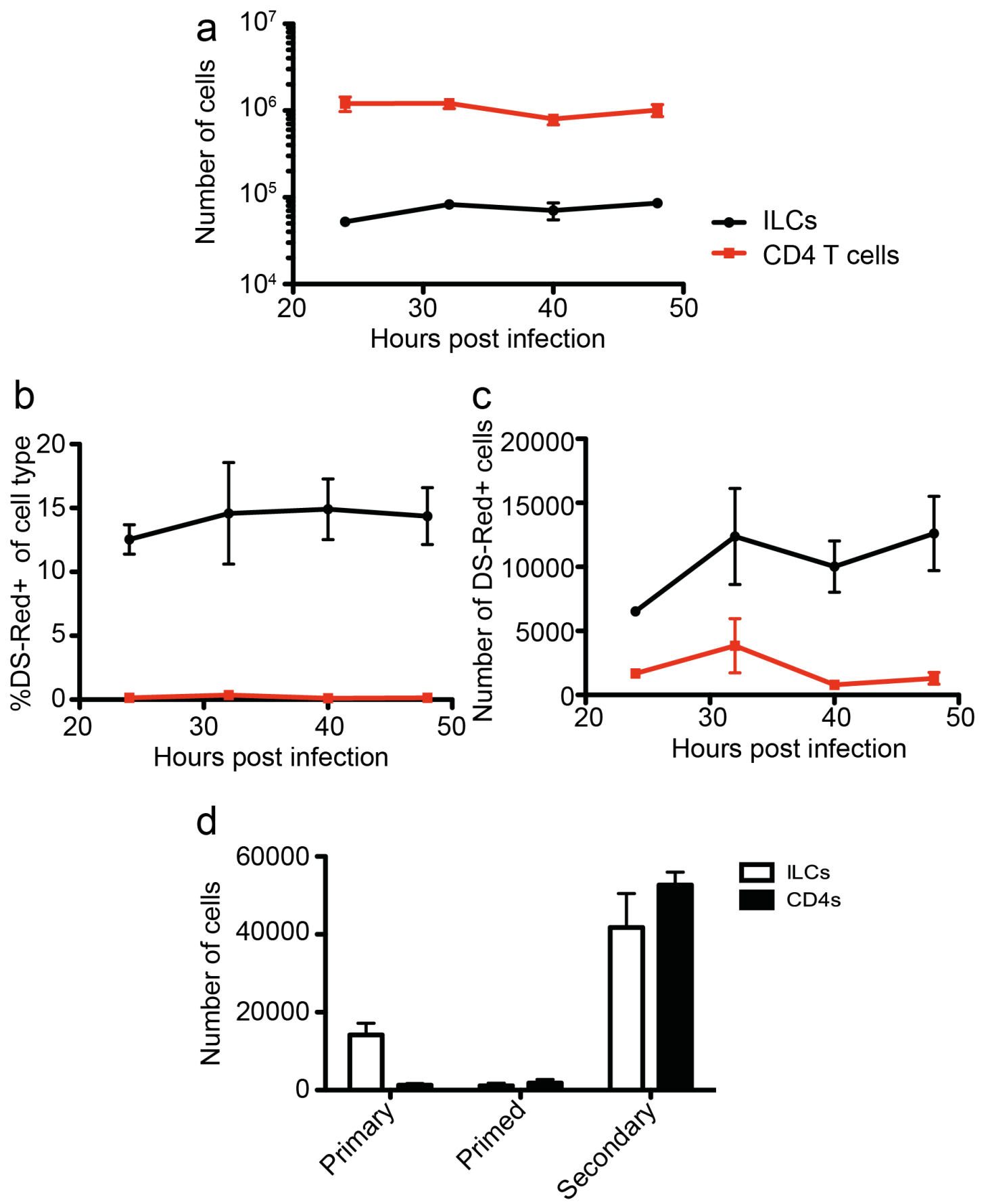

Figure 6.4 - ILC2s respond quickly to a primary $N$. brasiliensis infection and match $T$ cell IL-13 responses in a secondary infection.

a. total number, b. proportion expressing DS-Red and c. number of DS-Red expressing ILC2s and $\mathrm{CD}^{+} \mathrm{T}$ cells from digested whole lungs between 24 and 48 hours post primary i.v. $\mathcal{N}$. brasiliensis infection. Graphs show mean \pm S.E.M, $\mathrm{n}=3$ in each group. Data is from 2 independent experiments. d. Numbers of DS-Red expressing ILC2s and CD4 ${ }^{+} \mathrm{T}$ cell from digested whole lungs from mice 2 days after primary infection, 30 days post-primary (primed) and 2 days after secondary infection. Bar graphs show mean \pm S.E.M, $n=3$ in each group. Data is from a 2 independent experiments. 
$\mathrm{CD}^{+} \mathrm{T}$ cells are critically important for optimal protection, with mice that are deficient in $\mathrm{CD}^{+} \mathrm{T}$ cells such as $\mathrm{MHC}$ class $\mathrm{II}^{-/-}$having significantly reduced levels of protection ${ }^{132}$. To measure the importance of $\mathrm{CD}^{+} \mathrm{T}$ cells at different phases of infection, we used GK1.5 antibody to deplete $\mathrm{CD}^{+} \mathrm{T}$ cells prior to primary and secondary infection. The effectiveness of the antibody was tested and found that CD4 ${ }^{+}$ $\mathrm{T}$ cells in the lung are totally depleted 24 hours after i.p. injection and a single $0.5 \mathrm{mg}$ injection was sufficient to maintain depletion for at least 3 days (Figure 8.2). Using this depletion technique the importance of $\mathrm{CD}^{+} \mathrm{T}$ cell for both priming and during the active protection phase could be assessed. $\mathrm{CD}^{+} \mathrm{T}$ cells were depleted 1 day prior to primary infection then the number of lung larvae 2 days after the secondary infection were compared to undepleted mice. $\mathrm{CD} 4{ }^{+} \mathrm{T}$ cell depletion during primary infection did affect the number of surviving larvae in a secondary exposure with a significant increase in larval number compared with normal secondary infection (Figure 6.5a). However $\mathrm{CD}^{+} \mathrm{T}$ cells depleted 1 day before secondary infection found that there was no difference in protection, with normal low levels of larvae recovered from the depleted lungs. From this data it was evident that $\mathrm{CD}^{+} \mathrm{T}$ cells had a role in priming the protective responses, with their transient depletion during primary infection leading to sub-optimal protection in a secondary. However we found they were not required during the protective response, as their depletion just prior to reinfection had no effect on the number of recovered larvae.

Another depletion method was trialled where $\mathrm{CD} 4^{+} \mathrm{T}$ cells were depleted a week before the secondary infection. To maintain the depletion a $0.5 \mathrm{mg}$ injection of GK1.5 antibody was given 7 days before the secondary infection then a booster injection at day 3 prior to infection, this regime ensured $\mathrm{CD} 4^{+} \mathrm{T}$ cell depletion was maintained in lung until 2 days post infection. Mice depleted for the week before secondary infection had an entire reversal of protection with high larval numbers equal to counts from primary infected mice (Figure 6.5a). So while the presence of CD4 ${ }^{+} \mathrm{T}$ cells during the secondary infection were not required for protection they do have a role in maintaining the protective environment, with a sustained absence leading to a loss of the protective responses they support. 
a

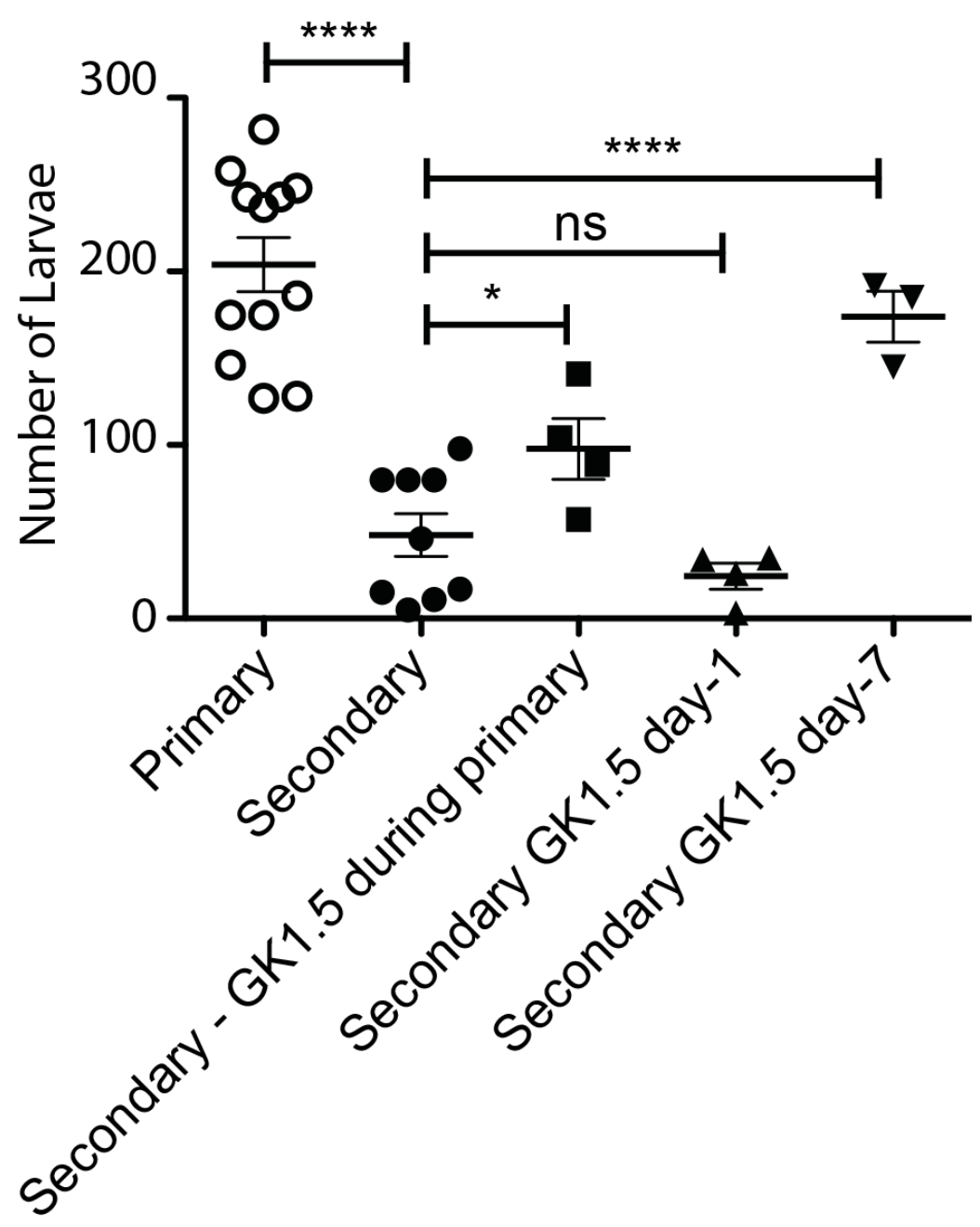

Figure 6.5 - GD4 T cells are required for long term protection but are not effector cells of the immune response.

a. Number of $\mathcal{N}$. brasiliensis larvae in the lungs of mice 2 days post primary i.v. infection (Primary) and i.v. reinfection (Secondary), or after $\mathrm{CD}^{+} \mathrm{T}$ cell depletion before primary infection (Secondary - GK1.5 during primary), and 24 hours or 7 days before secondary infection (Secondary GK1.5 day-1 and Secondary GK1.5 day-7 respectively). Graph shows mean \pm S.E.M. in each group. Data are representative of 3 independent experiments. Statistics were calculated for each group using One way ANOVA with Bonferroni multiple comparisons post test $* \mathrm{P}<0.05 * * * * \mathrm{P}<0.0001 \mathrm{~ns}=$ not significant. 
An increase in ILC2 number was observed between primary and secondary infection (Figure 6.4b). In secondary infected mice that had their $\mathrm{CD} 4^{+} \mathrm{T}$ cells depleted for 1 week before infection a significant decrease in ILC2 numbers was recorded (Figure 6.6a). CD4 depletion also led to a non-significant decrease in the number of DS-Red ${ }^{+}$ ILC2s(Figure 6.6b) and a decrease in their expression of CD25(Figure 6.6c).

These data highlights the responses by $\mathrm{CD}^{+} \mathrm{T}$ cells and ILC2s to $\mathcal{N}$. brasiliensis infection. $\mathrm{CD}^{+} \mathrm{T}$ cells are slower to respond than ILC2s in a primary infection but both populations can be detected producing cytokines at day 2 of a secondary infection. We have shown that $\mathrm{CD}^{+} \mathrm{T}$ cells are required to set up and maintain the protective environment, their long-term absence leading to a total loss of protection, but they are not required for the active protective mechanism. ILC2 numbers, and their activation based on DS-Red and CD25 expression, are dependent upon CD4 ${ }^{+} \mathrm{T}$ cells, with long term $\mathrm{CD}^{+}{ }^{+} \mathrm{T}$ cell depletion leading to decreases in ILC2 responses. 
a

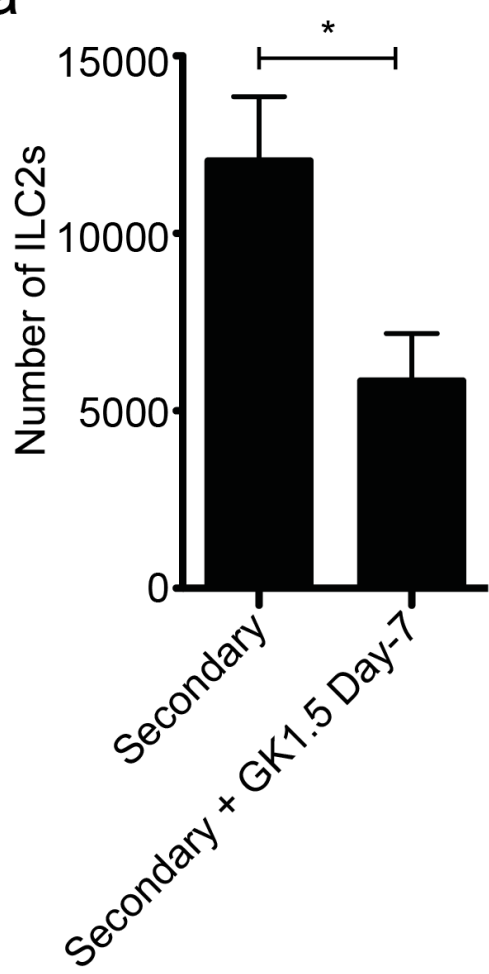

b

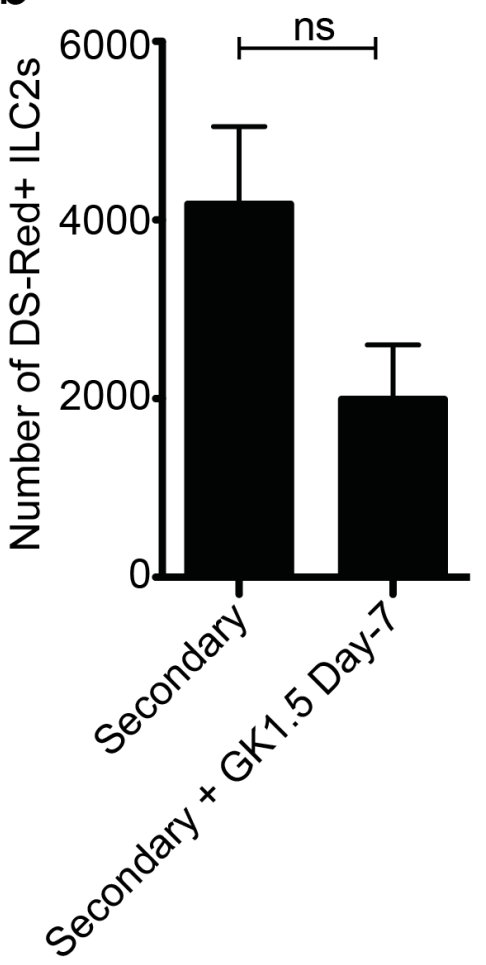

C

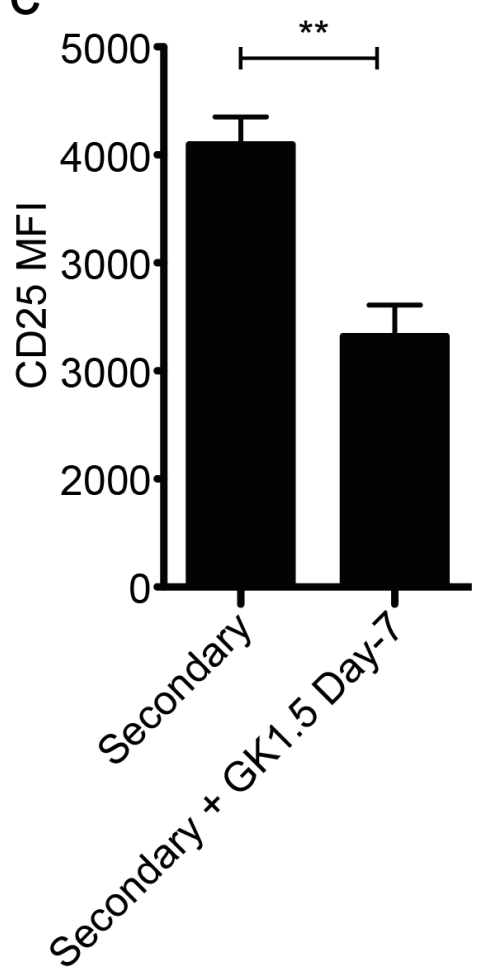

Figure 6.6 - Secondary ILC2 responses are decreased in the absence of GD4 T cells.

Number of a. total ILC2s and b. DS-Red expressing ILC2s and c. CD25 mean fluorescent index (MFI) of ILC2s from the digested whole lungs 2 days after secondary i.v. infection with or without treatment with aCD4 antibody (GK1.5) 7 days (Day-7) preceding the secondary infection. Bar graphs show mean \pm S.E.M. $\mathrm{n} \geq 4$ in each group. Data are representative of two independent experiments. Statistics were calculated for each group using Student's t test $* \mathrm{P}<$ $0.05 * * \mathrm{P}<0.01 \mathrm{~ns}=$ not significant. 


\subsubsection{ILC2 stimulating cytokines can induce protective immune responses in naïve mice}

With the evidence that ILC2s were quick responders to primary infection, and that $\mathrm{CD}^{+} \mathrm{T}$ cells were required for optimum ILC2 responses in the secondary infection, we wanted to assess the mechanisms activating ILC2s during infection. Barrier-derived cytokines have been implicated in ILC2 activation in response to $\mathcal{N}$. brasiliensis ${ }^{252,353}$. Previous work from our lab had shown that IL-25 was not important in the protective immune responses ${ }^{360}$ and as the IL-33R (ST2) had been detected on the lung ILC2s, along with the fact that IL-33/- mice have deficient protective responses ${ }^{353}$. The role of IL-33 activated ILC2s in protection was assessed. We also selected IL-2 to be tested as an activator of ILC2s because the ILC2s expressed the IL-2R $\alpha$ (CD25) and this could be a potential link between activated $\mathrm{CD}^{+} \mathrm{T}$ cells and ILC2 maintenance ${ }^{358}$. For efficient in vivo treatment with IL-2, IL-2 was complexed with its cognate antibody (IL2c), increasing the half-life and only allowing signalling through the high affinity CD25 inclusive receptor ${ }^{301,361}$. Lung ILC2 responses to these cytokines were tested by treating 4G13R mice intranasally (i.n.) with IL-33 alone, or i.p. with either IL-2 blocking antibody or IL-2c, and assessed numbers of, and DS-Red expression by ILC2s 24 hours after treatment. We found that IL-33 alone, or with IL-2 blocking antibody, did not affect the numbers of ILC2s in the lung within 24 hours of treatment (Figure 6.7a) though both treatments did lead to expansion of the percentage expressing DS-Red (Figure 6.7b) and thus the number of these cells too (Figure 6.7c). As has been demonstrated in vitro ${ }^{362}$, IL-2c and IL-33 dual treated mice had increased numbers of ILC2s and significantly increased proportions and numbers of IL-13 reporting ILC2s to a level suggesting an additive interaction between these two cytokines on ILC2 responses. From this we found that both IL-33 and IL-2c could rapidly activate ILC2s and led to their increased IL-13 production. 


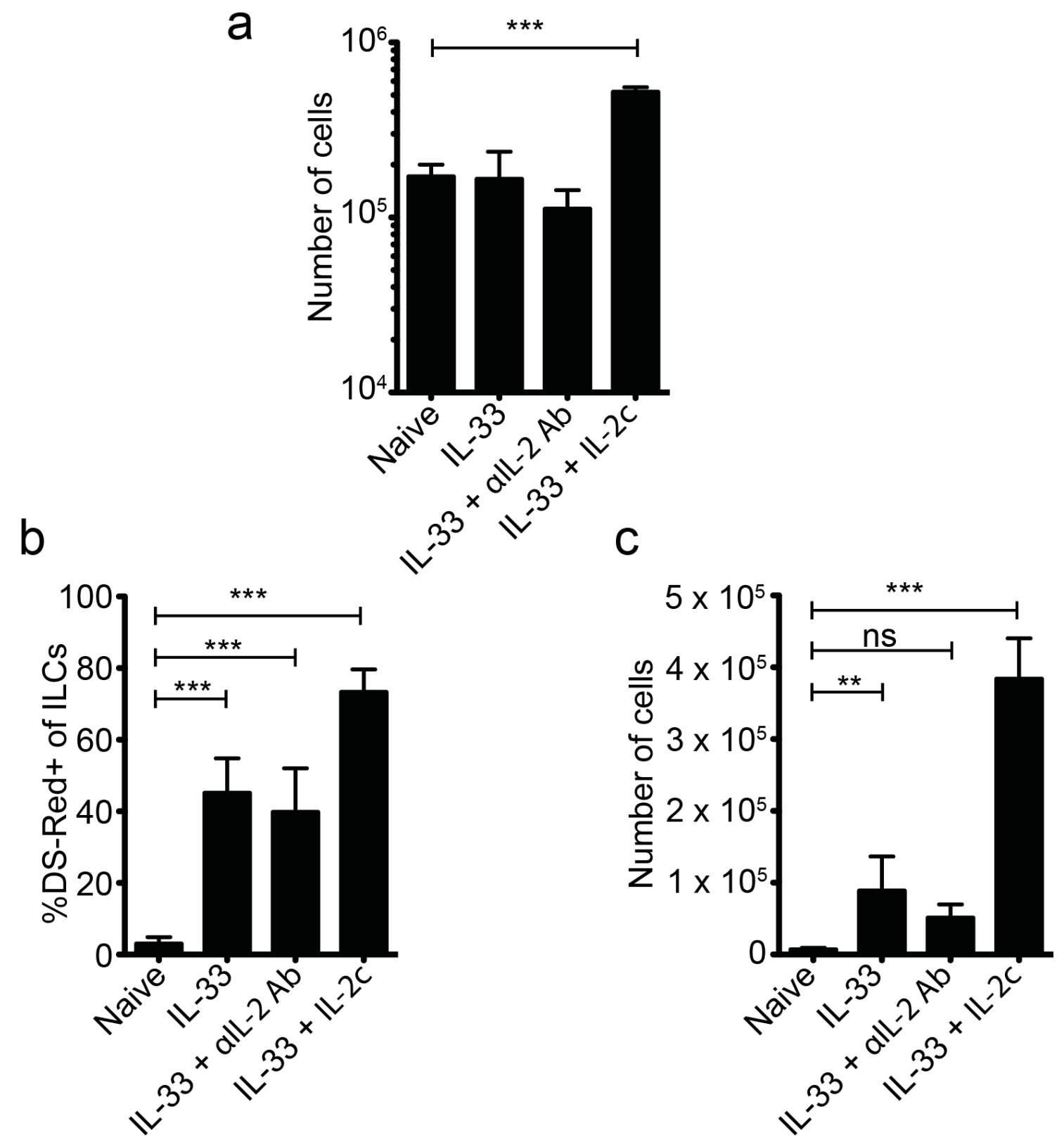

Figure 6.7 - Lung ILG2s are responsive to IL-33, IL-2c and combinations of cytokine.

a. Number of total ILC2s and b. proportion of DS-Red expressing ILC2s and c. numbers of DS-Red expressing ILC2s from the digested whole lungs of naïve WT mice or mice treated 24 hours prior with intranasal $0.5 \mu \mathrm{g}$ IL-33 with and without aIL-2 antibody (48 hours prior to harvest) or IL-2/aIL-2 antibody complex (IL-2c, 72 and 24 hours prior to harvest). Bar graphs show mean \pm S.E.M. $\mathrm{n} \geq 3$ in each group. Data are representative of three independent experiments. Statistics were calculated for each group using One way ANOVA with Tukey's post test $* \mathrm{P}<0.05 * * \mathrm{P}<0.01 * * * \mathrm{P}<0.001 \mathrm{~ns}=$ not significant. 
To determine if IL-33 driven expansion of ILC2s would be sufficient to protect against $\mathcal{N}$. brasiliensis a model was established where naïve mice were treated i.n. with IL-33, 2 days later challenged i.v. with 550 L3 larvae and lung larvae numbers were enumerated on day 2 post infection. CD4 ${ }^{+} \mathrm{T}$ cells were also depleted from an IL-33 treated group to assess the requirement for $\mathrm{CD}^{+} \mathrm{T}$ cells. IL-33 treatment alone induced protection against primary infection in naïve mice and depletion of $\mathrm{CD}^{+} \mathrm{T}$ cells with IL-33 enhanced this protective response (Figure 6.8a). IL-33 treatment prior to primary infection led to increases in ILC2 numbers, intriguingly GK1.5 treatment abrogated the ILC2 expansion.

The discovery that cytokine treatment could drive protective immune responses led Dr Tiffany Bouchery to compare the mechanisms involved in IL-33 driven protection to the mechanisms that mediate protection in secondary infection. As with secondary infection macrophages were expanded and were also critical to IL-33 driven protection $^{354}$. When STAT6 and IL-4 deficient mice were treated with i.n. IL-33 and i.p. GK 1.5 the IL-4 deficient mice were protected but the STAT6-/ were not, indicating that this IL-33 model, like secondary infection, required STAT6 signalling independent of IL-4 ${ }^{354}$. This indicated that as in secondary infection, in the absence of IL-4 IL-13 could drive protective responses. Importantly it also ruled out the possibility of IL-33 directly activating a population of cells, such as M2 activated macrophages, that could provide protection against infection. Unlike in natural secondary infection IL-33 treatment was able to induce protection in $\mathrm{Rag} 1^{1 /-}$ mice. 
a

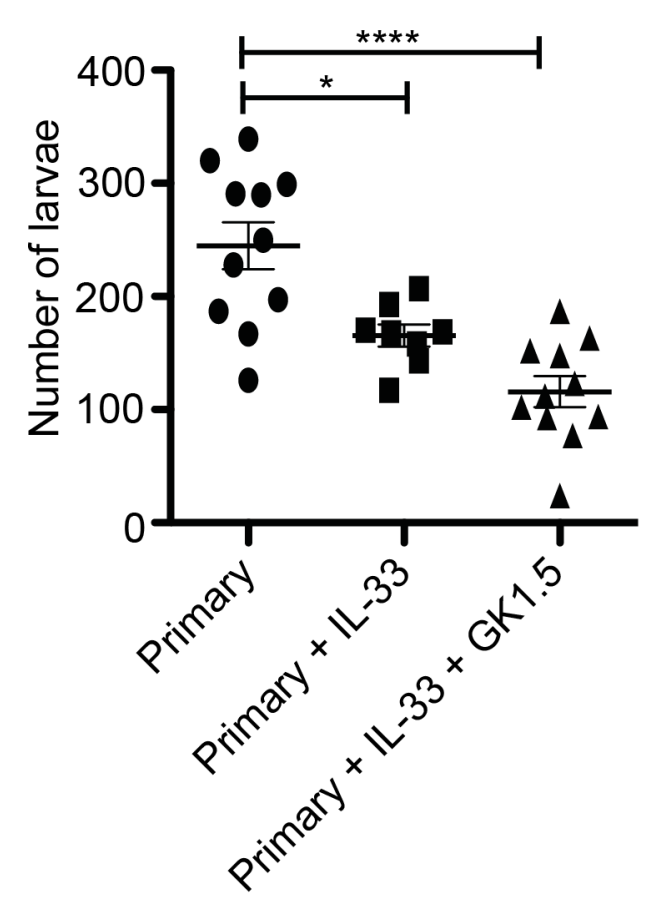

b

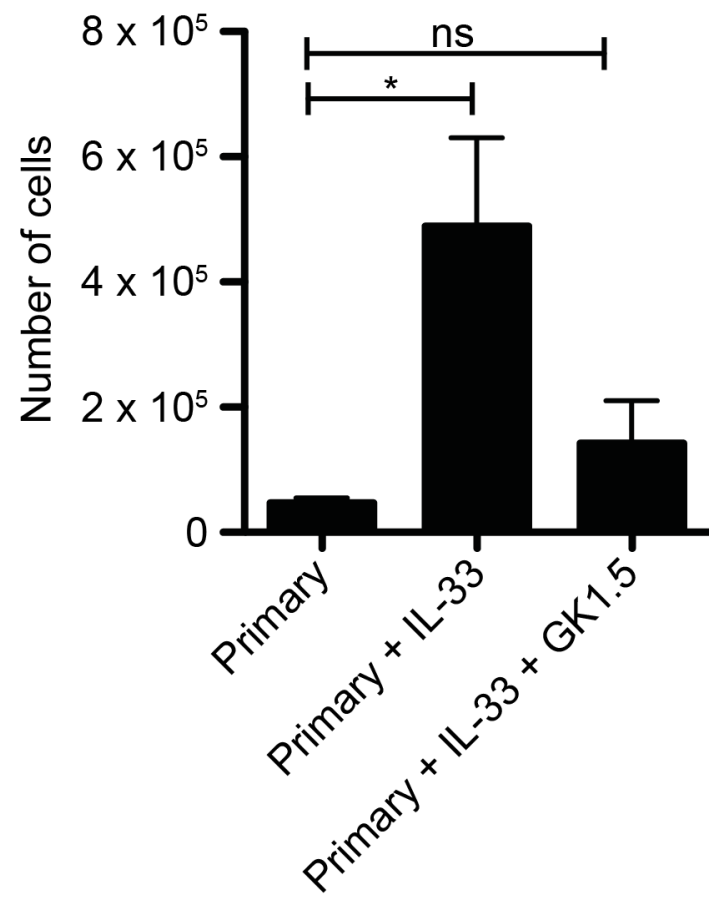

Figure 6.8 - IL-33 treatment can provide protection in the absence of GD4 $\mathrm{T}$ cells. a. Number of larvae and $\mathbf{b}$. number of ILC2s from lungs 2 days post-primary $\mathcal{N}$. brasiliensis infection of untreated mice (primary) and mice treated with $0.5 \mu \mathrm{g}$ IL-33 intranasally 2 days before infection with and without aCD4 antibody GK1.5 at the same time as IL-33 treatment. a. Plots show mean \pm S.E.M. of each group. Data is pooled from 4 experiments. Statistics were calculated for each group using One way ANOVA with Tukey's post test $* \mathrm{P}<0.05 * * * * \mathrm{P}<$ 0.0001. b. Bar graphs show mean \pm S.E.M. $n \geq 3$ in each group. Data are representative of three independent experiments. Statistics were calculated for each group using One way ANOVA with Tukey's post test $* \mathrm{P}<0.05 \mathrm{~ns}=$ not significant. 
I repeated the IL-33 protection model but replaced i.n. IL-33 with i.p. IL-2c. Treatment with IL-2c was sufficient to induce protective immune responses in the absence of $\mathrm{CD}^{+} \mathrm{T}$ cells (Figure 6.9a), and also expanded ILC2 numbers (Figure 6.9b). IL-2c treatment also induced protection in Rag 1/- mice. To assess if ILC2s were the IL2 responding cells that were mediating the protective immune responses, ILC2s were depleted with a regime requiring multiple treatments of $\alpha \mathrm{CD} 90$ antibody in $\mathrm{Rag} 1^{-/}$. Treatment with $0.5 \mathrm{mg}$ of $\alpha \mathrm{CD} 90$ antibody (30H12) daily for 5 days prior to infection, and the day after infection was sufficient to maintain depletion until 2 days after infection in most mice. Worms were collect by bronchial alveolar lavage for counting while lung tissue was digested to assess ILC2 depletion, only worm counts were included from mice where ILC2 numbers were reduced below 50\% of undepleted mice. When this depletion was applied with IL-2c treatment to Rag $1^{-/-}$mice that were subsequently infected with $\mathcal{N}$. brasiliensis, protection was reversed (Figure 6.10c). This provided strong evidence that ILC2 populations were sufficient to provide protection when stimulated by cytokine. 
a

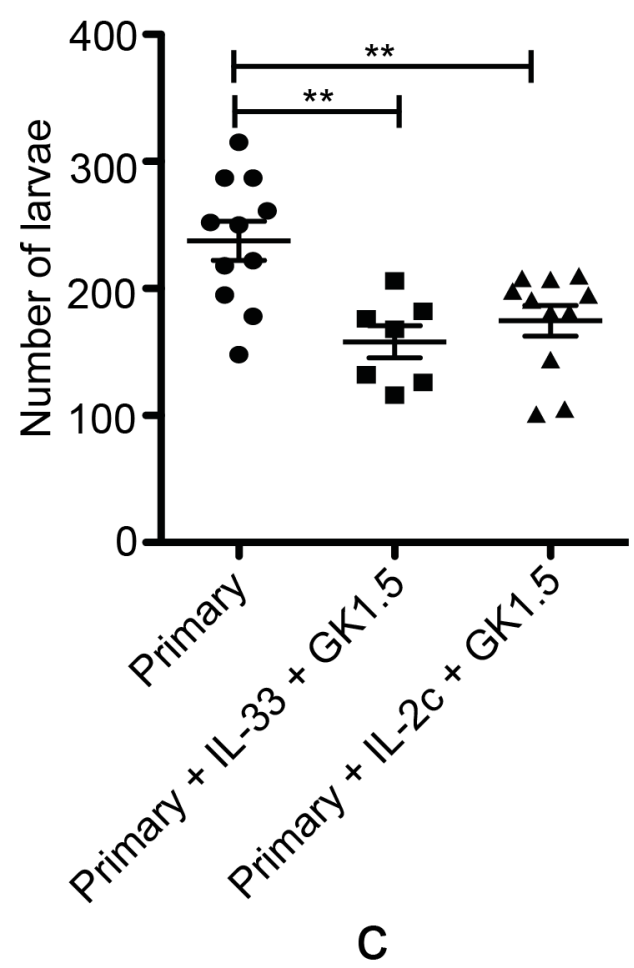

b

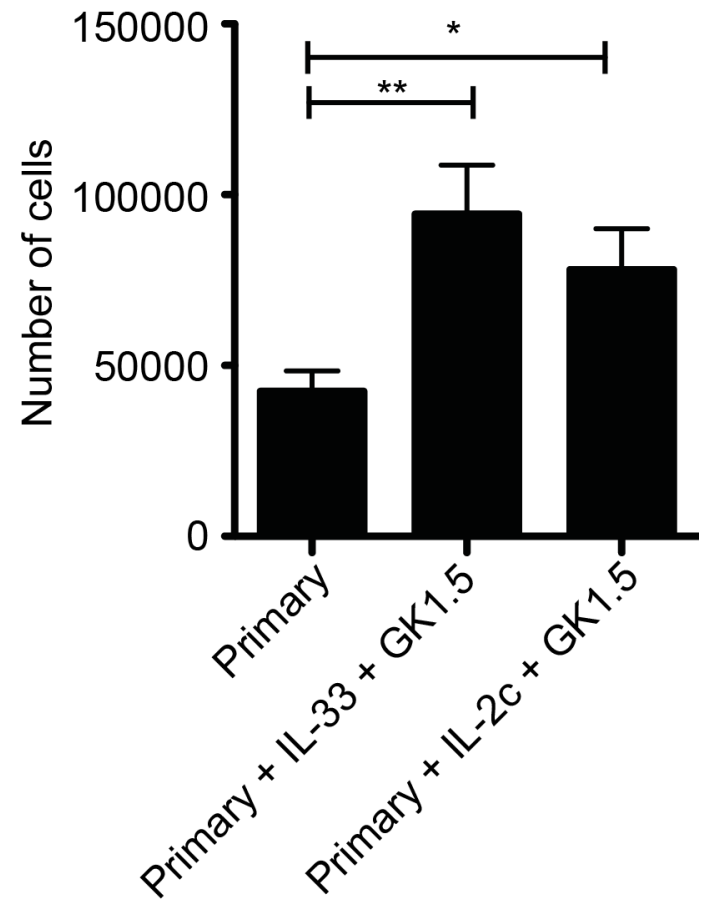

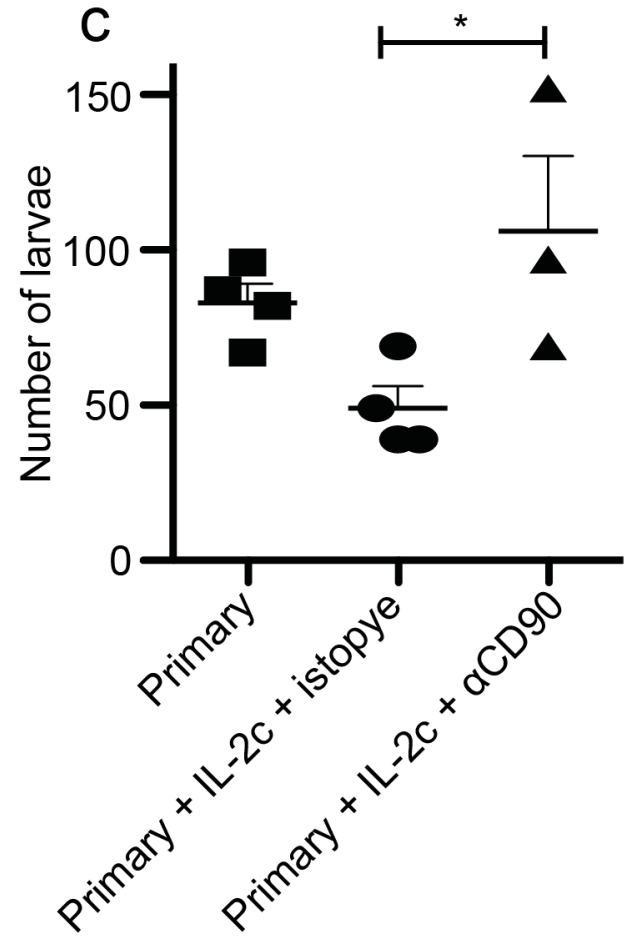

Figure 6.9 - IL-2c activated ILC2s are required for protective responses.

a. Number of larvae and $\mathbf{b}$. number of ILC2s from lungs 2 days post-primary $\mathcal{N}$. brasiliensis infection of untreated WT mice (primary), mice treated with GK1.5 and intranasal IL-33 (Primary + IL-33 + GK1.5) and mice treated with GK1.5 and i.p. IL-2c (Primary + IL-2c + GK1.5), antibody and cytokine treatments were given 2 days prior to infection. c. number of ILC2s from lungs 2 days post-primary $\mathcal{N}$. brasiliensis infection of untreated Rag1/- mice (Primary) or Rag 1/- mice treated with IL-2c and either isotype or $\alpha \mathrm{CD} 90$ antibody 2 days prior to infection. All plots show mean \pm S.E.M. of each group. Data are representative of two independent experiments. Statistics were calculated for each group using One way ANOVA with Tukey's post test * $\mathrm{P}<0.05$ ** $\mathrm{P}<0.01$ *** $\mathrm{P}<0.001$ **** $\mathrm{P}<0.0001$. 
Knowing that $\mathrm{T}$ cell independent protection could be achieved with IL-2c treatment, we decided to see if cytokine treatment could rescue the protection that is lost in a secondary infected mouse when $\mathrm{CD}^{+} \mathrm{T}$ cells are depleted 7 days prior to infection. The $\mathrm{CD}^{+}{ }^{+} \mathrm{T}$ cells were depleted as before (Figure 6.5) and the mice treated with IL-2c every 2 days during the depletion. IL-2c treatment rescued the protection lost with $\mathrm{CD}^{+} \mathrm{T}$ cell depletion and actually led to increased protection compared to natural secondary infection (Figure 6.10a). The long-term IL-2c treatment also caused massive expansion of the ILC2s (Figure 6.10b).

Therefore we found that the cytokines IL-33 and IL-2c cause rapid activation and eventual expansion of ILC2s, and these cytokines could induce $\mathrm{T}$ cell independent protection. The IL-2c driven protection was ILC2 dependent as depletion with $\alpha$ DC90 abrogated the protection. Finally IL-2c treatment caused massive expansion of ILC2s in secondary infected mice that had their $\mathrm{CD} 4^{+} \mathrm{T}$ cells depleted, and this treatment was able to restore the protection despite the long-term absence of $\mathrm{CD} 4^{+} \mathrm{T}$ cells. 
a

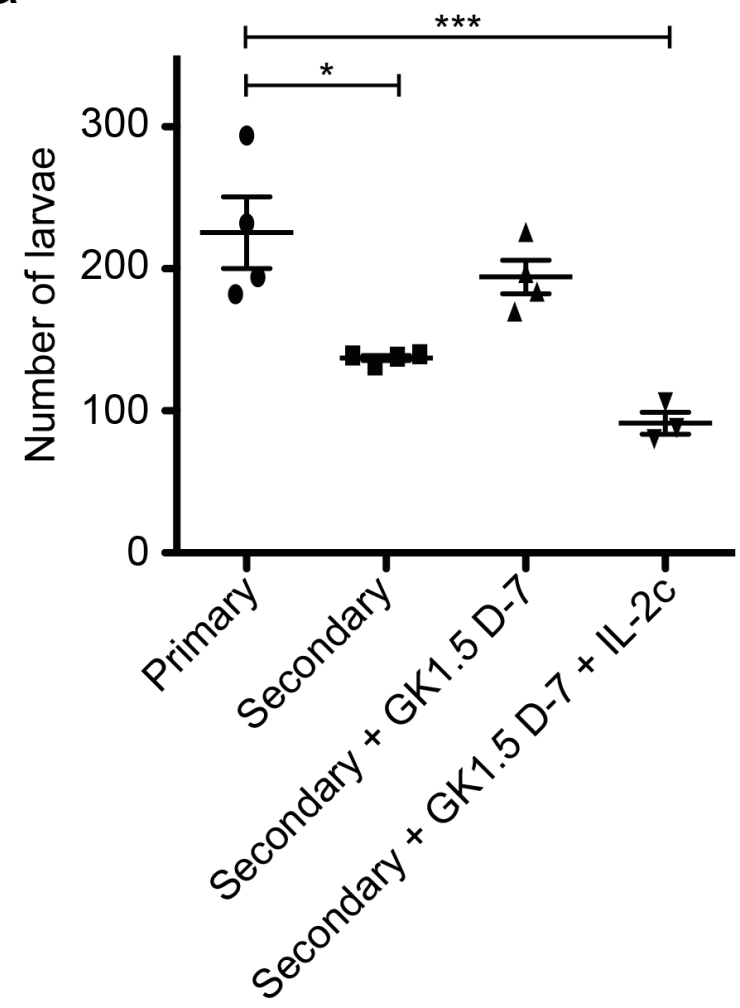

b

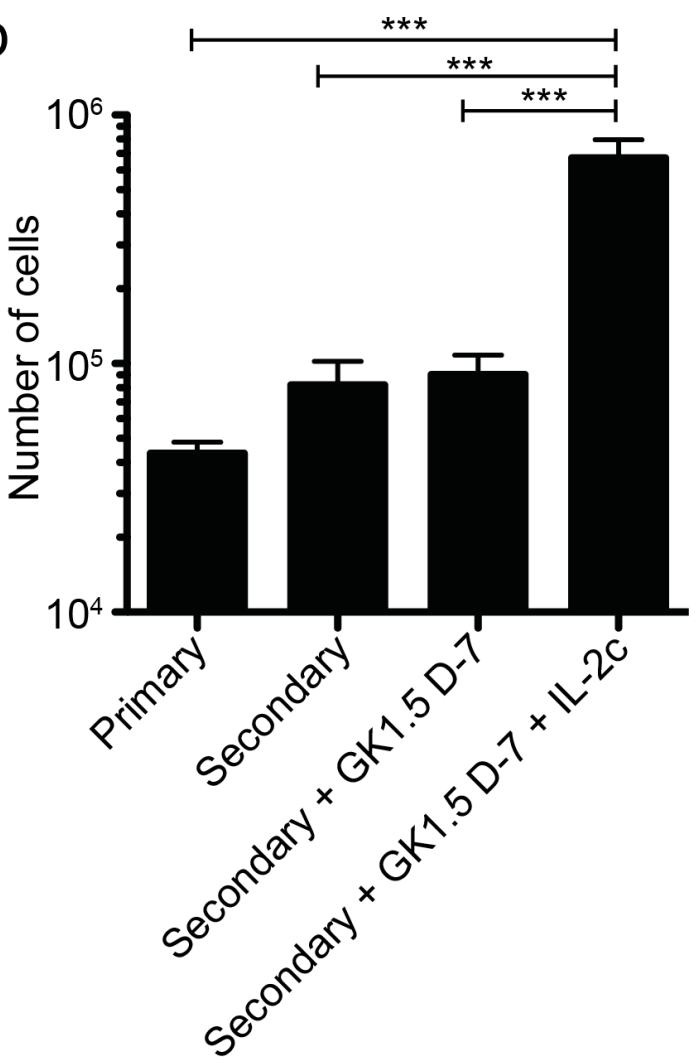

Figure 6.10 - Treatment with IL-2c rescues protection in the absence of GD4 T cells.

Number of a. larvae and b. ILC2s from the digested whole lungs 2 days after primary infection, secondary infection with and without treatment with aCD4 antibody (GK1.5) 7 days (Day -7) preceding the secondary infection and secondary infected mice that received IL-2c every 2 days during the 7 day CD4 depletion period. Plots show mean \pm S.E.M. $n=4$ each group. Data are representative of two independent experiments. Statistics were calculated for each group using One way ANOVA with Tukey's post test $* \mathrm{P}<0.05 * * * \mathrm{P}<0.001$. 


\subsection{Discussion}

The focus of this chapter was to elucidate the immune cells, and the signalling pathways employed by these cells, that are critical for protection against $\mathcal{N}$. brasiliensis. The results I have found, alongside other research within this laboratory, are the first to conclusively show that $\mathrm{CD} 4{ }^{+} \mathrm{T}$ cells and ILC2s contribute to protective responses in the lung by maintaining M2 activation of macrophages through the production IL-4 and IL-13. These cells and signalling pathways collectively mediate larval arrest and killing within the lung 354 .

IL-13 production by ILC2s has been widely reported including in the lung in models of allergic airways inflammation ${ }^{271}$ and even $\mathcal{N}$. brasiliensis infection ${ }^{358}$. Previous hookworm infection studies required in vitro culture and restimulation of lung cells to identify cytokine producers with intracellular staining ${ }^{358}$. By using the reporter mouse system to detect real time cytokine production directly ex vivo I have been able to make more direct interpretations about cytokine production by different cell populations. Identification of IL-13 reporting ILC2s as early as 12 hours post infection indicates these cells respond much faster than $\mathrm{CD}^{+} \mathrm{T}$ cells during the primary infection, though during a secondary infection robust cytokine production by day 2 post infection demonstrates rapid memory response of the CD4+ $\mathrm{T}$ cells (Figure 6.4). ILC2s consist of a much smaller population of cells when compared to total $\mathrm{CD}^{+} \mathrm{T}$ cells in the lung. Their relatively small numbers compared with other immune cell populations has often drawn debate as to how influential they could be to an immune response, particularly one that involves a strong $\mathrm{CD} 4{ }^{+} \mathrm{T}$ cell response as well. The discovery that in a secondary infection there are similar numbers of IL-13 producing ILC2s and CD4 ${ }^{+} \mathrm{T}$ cells is evidence that when compared to antigen specific cytokine responses, ILC2s can contribute significantly to the overall response, though it remains to be assessed the relative amount of cytokine produced per cell from each of these populations.

$\mathrm{CD}^{+} \mathrm{T}$ cells are required for optimal immunity, and adoptive transfer of $\mathrm{CD} 4^{+} \mathrm{T}$ cells from the lung of a previously infected host can convey protection ${ }^{354}$. In this study I have demonstrated a more nuanced role for these cells in protective responses. These data indicate that $\mathrm{CD} 4^{+} \mathrm{T}$ cells are not required for effector functions against the larvae as protection is maintained when $\mathrm{CD} 4{ }^{+} \mathrm{T}$ cells are depleted only one day before infection. However, the importance of $\mathrm{CD} 4^{+} \mathrm{T}$ cells is exemplified by the loss of protection with longer term (seven day) depletion, indicating that $\mathrm{CD}^{+} \mathrm{T}$ cells are critical for 
maintaining other effector cell populations capable of dealing with the invading parasites such as M2 macrophages.

Seven day depletion of $\mathrm{CD} 4^{+} \mathrm{T}$ cells also resulted in decreased numbers of ILC2 and decreased IL-13 reporting ILC2s during secondary infection. Based on this evidence $\mathrm{CD}^{+} \mathrm{T}$ cells positively influence lung ILC2 responses in vivo. While it has been shown that ILC2s and CD4 ${ }^{+} \mathrm{T}$ cells can communicate through MHC II - TCR interaction, previous in vivo studies focussed on the effects of these interactions on the $\mathrm{CD} 4^{+} \mathrm{T}$ cell ${ }^{358}$. However it has been noted that adoptive transfer of $\mathrm{MHC} \mathrm{II}^{-/}$ILC2s leads to diminished expulsion of $\mathcal{N}$. brasiliensis from the gut compared to transfer of WT ILC2s in a model where these ILC2s are the only IL-13 expressing cell type ${ }^{363}$. This study does not directly identify the nature of the CD4 ${ }^{+} \mathrm{T}$ cell ILC2 interaction within the lung but establishes a clear relationship whereby $\mathrm{CD}^{+} \mathrm{T}$ cells support ILC2, as well as M2 macrophage responses. This may also explain why ILC2s cannot maintain M2 macrophage phenotype in the absence of $\mathrm{CD}^{+} \mathrm{T}$ cells, as the ILC2s themselves are also missing a maintenance signal. Addition of IL-2c to the $\mathrm{CD} 4^{+} \mathrm{T}$ cell depleted mice rescued (actually expanded) ILC2 numbers and cytokine production, which led to maintenance of M2 macrophages and protective responses. IL-2 is released by activated $\mathrm{T}$ cells and is a potential communication signal between CD4+ $\mathrm{T}$ cells and ILC2s. It has been shown in vitro GD4 $\mathrm{T}$ cell derived IL-2 can activate ILC2s inducing strong cytokine production, however in these studies, a proliferative response from the ILC2s was not observed, This may be due to limited amounts of IL-2 produced by CD4 ${ }^{+} \mathrm{T}$ cells, or the lack of a proliferation promoting accessory signal that is present in vivo ${ }^{363}$. A recently published paper has identified a population of lung resident ILC1s or ILC3s as a potential source of IL-2 ${ }^{364}$, the responses of these cells during $\mathcal{N}$. brasiliensis infection remain to be assessed but these cells could also be contributing to the activation of ILC2s through the production of IL-2.

ILC2s in the absence of $\mathrm{CD}^{+} \mathrm{T}$ cells could not provide protection in a natural immunity setting, though when sufficiently stimulated with the cytokines IL-33 or IL-2, protective responses could be established in a $\mathrm{T}$ cell independent manner. It is of interest that protective responses established by IL-33 treatment were more efficient in the absence of $\mathrm{CD} 4^{+} \mathrm{T}$ cells. Tregs have been shown to respond to IL-33365 and could potentially have an effect of diminishing protective immune responses, however a single observation using FoxP3-GFP reporter mice to identify Tregs demonstrated no significant increase of Tregs with IL-33 treatment (unpublished data). IL-33 has also 
been implicated in driving a M2 macrophage phenotype directly ${ }^{366}$, however as the IL33 protective response required STAT6 signalling this ruled out the possibility of IL-33 bypassing the IL-13 producing ILC2s and directly activating macrophages. As I had previously found in the skin, there were significant difficulties depleting ILC2s from the lung via antibody depletion methods; using the IL-33 model antibody treatment never established depletion of ILC2s to a level where their requirement for the IL-33 induced protection could be assessed.

IL-33 is required for optimal ILC2 IL-13 expression in response to $\mathcal{N}$. brasiliensis infection $^{353}$, so it was expected that treatment with IL-33 would induce strong IL-13 expression by the lung ILC2s. IL-33 has been shown to be required for secondary responses as well, however larvae counts were taken from the intestines so from these data it was unknown whether IL-33 was required for gut expulsion or protective responses earlier in the transit of the host ${ }^{353}$. While I haven't been able to assess if IL-33 is required, I have demonstrated that lung administration of IL-33 is sufficient to induce protective responses. Not only was IL-2c treatment able to stimulate ILC2 responses in the absence of $\mathrm{CD}^{+} \mathrm{T}$ cells and maintain protection in a secondary infection, IL-2c also stimulated protective immune responses in naïve mice in a T cell independent way. Unlike the IL-33 model, antibody depletion of ILC2s could be achieved with the IL-2c treatment, allowing the requirement of ILC2s in this model to be shown. This study is the first to demonstrate that ILC2s in the lung are sufficient to mediate protective responses against $\mathcal{N}$. brasiliensis infection.

A paper published during the course of this project came to similar conclusions regarding the role of IL-13 and M2 macrophages in protective responses, however this paper attributed the cellular source of IL-13 to neutrophils ${ }^{367}$. Ly6G targeting antibody depletion led to diminished M2 macrophage activation and protective responses, and neutrophils sorted from $\mathcal{N}$. brasiliensis infected hosts had mRNA signals for IL-13 expression. Co-culture of neutrophils from $\mathcal{N}$. brasiliensis infected donors caused macrophages to display M2 characteristics, which could be blocked with the administration of IL-13 blocking antibodies ${ }^{367}$. Experiments performed by Dr Tiffany Bouchery in our lab have found that depletion with an antibody with a broader specificity, Ly6C/Ly6G specific Grl clone, had no effect on protective responses in this i.v. model of infection ${ }^{354}$. Furthermore I have not detected IL-13 reporting from neutrophils or any $\mathrm{SSC}^{\text {hi }}$ population in these experiments (data not shown). Depletion of neutrophils does affect protective responses when reinfection is administered 
subcutaneously only 4 days after primary infection. In this model it is conceivable that rapidly recruited neutrophils play a role in protective responses in the skin ${ }^{354}$. Why such different requirements for neutrophils have been demonstrated between our study and the one carried out by Chen et al. remains unknown.

Through adoptive transfer and depletion experiments run concomitantly with my work it was shown that M2 macrophages are both sufficient and required for protection against $\mathcal{N}$. brasiliensis infection within the lung ${ }^{354}$. Macrophages initiate the M2 programme after exposure to either IL-4 or IL-13; they express the IL-4R $\alpha$, IL-13R $\alpha$ I and $c \gamma$ chains so are able to respond through both receptor complexes ${ }^{185,} 368,369$. Through the production of IL-4 and IL-13 CD4 ${ }^{+} \mathrm{T}$ cells and ILC2s support M2 macrophage generation and maintenance within the lung post infection, sustaining the M2 phenotype to protect from future parasitic infections. IL-4 deficient mice treated with $\alpha$ IL-13 antibody lost protection against reinfection indicating that these cytokines were the STAT6 dependent signal that drove protection. However the fact that both IL-4 deficient mice, and $\alpha$ IL-13 treated WT mice were protected in a secondary infection demonstrated strong redundancy in the system and, that the presence of either of these cytokines could sufficiently maintain M2 macrophage activation and the protective response. So while mechanisms exist for differentially regulating the expression of IL-4 and IL-13 in some cases their functions significantly overlap to the point that they are completely redundant, only when signalling by both cytokines is inhibited are the effects of their depletion observed.

\subsection{Conclusions}

I aimed to identify the key cells and signalling pathways that provide protection against reinfection by $\mathcal{N}$. brasiliensis. M2 macrophages are both sufficient and required to provide protection and the phenotype of these cells is maintained by IL-4 and IL-13 signalling, which $\mathrm{CD}^{+} \mathrm{T}$ cells and ILC2s are major producers of. There is almost complete redundancy in the protective responses maintained by IL-4 and IL-13. Depletion studies found that while $\mathrm{CD} 4^{+} \mathrm{T}$ cells were not effector cells they were critical for sustaining the long term protective environment by maintaining M2 macrophages. Although ILC2s expressed IL-13 they were not able to sustain protective responses in the absence of $\mathrm{CD}^{+} \mathrm{T}$ cells, this was partly due to the fact that their numbers and 
activation were also diminished upon $\mathrm{CD} 4^{+} \mathrm{T}$ cell depletion, possibly due to a lack of IL-2 signalling. Exogenous IL-2 expanded ILC2s to a level where they could sustain M2 macrophages and rescue protection in the absence of CD4 ${ }^{+} \mathrm{T}$ cells. IL-2 activation of ILC2s was also shown to be sufficient for protection in naïve mice, and IL-33 treatment similarly activated ILC2s and produced protective immune responses. This study demonstrated that IL-33 and CD4 ${ }^{+} \mathrm{T}$ cells, possibly through IL-2, support ILC2 responses in the lung, and together Type 2 cytokine producing ILC2s and CD4 ${ }^{+} \mathrm{T}$ cells types maintain M2 activation of macrophages that mediate protection against $\mathcal{N}$. brasiliensis reinfection. 


\section{General Discussion}

The purpose of the research outlined in this thesis was to increase our understanding of how the production of two key Type 2 cytokines, IL- 4 and IL-13, are regulated by both $\mathrm{CD}^{+} \mathrm{T}$ cells and Type 2 associated innate cell populations. These mechanisms have been investigated by utilising a novel transgenic dual reporter mouse in the context of in vivo models of skin inflammation and parasitic infection, as well as in vitro culture. Specifically I sought to understand the role of TCR signalling on the allelic expression of IL-4, and for the first time independently assess the impact of IL-4 on CD4+ ${ }^{+} \mathrm{T}$ cell IL-4 expression in vivo. I compared the mechanisms that control IL-13 expression by $\mathrm{CD}^{+} \mathrm{T}$ cells with those that control IL-4 expression. The expression of IL-4 and IL-13 by basophils and a novel population of dermal ILGs was assessed in a model of atopic dermatitis. Finally I characterised the roles of Type 2 cytokine producing lymphoid populations in the lung in protection against infection by $\mathcal{N}$. brasiliensis. The results of this study have highlighted significant differences in the mechanisms that regulate the expression of IL-4 and IL13; the following chapter will discuss the impacts of my findings in the context of the current literature.

\subsubsection{The allelic expression of IL-4 is controlled by multiple factors}

Cytokines including IL-2, IL-4 and IFN- $\gamma$ have dose dependent effects when stimulating immune cells ${ }^{47,222}$. The immune system must accurately regulate the amounts of these cytokines produced in vivo to limit negative consequences such as the inappropriate generation of $\mathrm{IgE}$ antibodies ${ }^{370}$. It has been suggested that stochastic mono-allelic 
expression is an effective way for the immune system to limit the total amount of a cytokine that is produced, rather than modulating the amount produced by each individual cell ${ }^{371}$. It has been found that in strong Th2 optimising conditions only a small proportion of $\mathrm{CD}^{+} \mathrm{T}$ cells actually express IL-4, with a $\sim 100$ fold increase in Il4 mRNA between IL-4 expressing and non-producing cells, despite their being similar GATA3 expression across the whole population ${ }^{372}$. The exact mechanisms which determine if, and from which combination of alleles, a CD4 ${ }^{+} \mathrm{T}$ cell will express IL-4 remain unknown. The accessibility of the IL-4 allele has been suggested as a potential method for allelic regulation ${ }^{372}$. The results I have generated are in agreement with previous work from Guo et al. where by restimulation of cells can lead to reassignment of which IL-4 allele may be expressed by $\mathrm{CD} 4^{+} \mathrm{T}$ cells (Figure 3.7). This data indicates that it is unlikely to be epigenetic modulation that silences alleles in recently differentiated mono-allelically expressing cells. If epigenetic mechanisms were in effect it would be expected that daughter cells would maintain their allelic phenotypes during successive replication cycles, as has been seen with some mono-allelically expressed genes controlled epigenetically by clones of neural progenitor cells ${ }^{373}$. These data also refute the theory that bi-allelically expressing cells represent the more terminally differentiated Th2 effector cells. Sorted CD4 ${ }^{+} \mathrm{T}$ cells expressing both IL-4 alleles had the same pattern of mono- and bi-allelic cells upon restimulation as $\mathrm{CD}^{+} \mathrm{T}$ cells expressing mono-allelically or not expressing IL-4 at the time of sorting. This shows that at least in terms of their IL-4 expression even bi-allelically reporting cells remain plastic for a while after differentiation.

Accessibility to DNaseI hypersensitivity sites $H S-I$ and $H S-I I I$ within the $I l 4$ gene have been observed in both IL-4 producing and non-producing cells ${ }^{374}$, however only IL-4 producing cells display increased accessibility to the $H S$ - $V_{A}$ site $3^{\prime}$ to the $4^{\text {th }}$ exon of the Il4 gene ${ }^{216}$. HS- $V_{A}$ has four binding domains for GATA3 and it has been proposed that binding of GATA3 to this site may be the stochastic event that determines IL-4 expression $^{371}$. While not measured in this thesis GATA3 stabilisation may explain the observed increase in bi-allelic expression with increasing TGR activation (Figure 3.5). It has been shown that TCR signalling stabilizes GATA3 expression partly through promoting Gfil, which is down stream of the MAPK signalling cascade ${ }^{375,376}$. Both IL4 and IL-13 are expressed allelically and it has been shown that there is no coordination of expression between an IL-4 allele and the IL-13 allele on the same chromosome. However the conserved noncoding sequence 1 (CNS1) on the same 
chromosome must be open, access is mediated by histone 3 acetylation, to permit expression of either cytokine. GATA3 has been shown to mediate access to the CNS1 site and this may be another site via which increased levels of GATA3 can modulate the chance of bi-allelic expression. ${ }^{226}$ So with increasing level of TCR signalling there should be increased stabilisation and availability of GATA3, which potentially could mediate bi-allelic expression. It remains to be shown if bi-allelically positive cells have higher GATA3 levels or if modulating GATA3 availability effects the proportion of Th2 cells expressing bi-allelically.

The data I have generated agree with past observations that IL-4 expression is a stochastic process with a low probability of occurring within a given cell. When it does occur the majority of the time only one allele is expressed although there is consistently a population that express bi-allelically (Figure 3.3). The foremost theory on why IL-4 is under such control is that it fine-tunes the number of IL-4 expressing cells is more efficient than regulating the amount of IL-4 produced by every cell. Having the chance of a cell actually expressing IL-4 very low ensures that only few cells will be expressing it at any one time. This can be altered by variables including, exposure to cytokines, differential TCR engagement and co-stimulation. This theory however does not explain the role for bi-allelically producing cells. It is possible that these cells represent the population of cells that can express simultaneously both alleles through random chance, and that the factors that generally affect the number of IL-4 expressing cells also increase the possibility of any given cell expressing from both alleles. In argument against this theory are the observations from past members of this lab that bi-allelically expressing cells also express higher levels of activation-associated molecules (unpublished data - Helen Mearns). This could be interpreted to mean that the biallelically expressing cells are a population of super-activated Th2 cells. It is possible that rather than random chance, bi-allelism can be induced by a particular combination of stimulatory signals, this theory is further supported by the observation that biallelically producing cells are more likely to express the markers of Tfh cells in the reactive LN than mono-allelic cells (unpublished data - Helen Mearns). This makes it tempting to infer that signals that promote Tfh differentiation may also drive bi-allelic expression, however the Tfh master transcription factor Bcl-6 is known to inhibit expression of GATA $3^{323}$ potentially conflicting with my earlier hypothesis that increased GATA3 could stabilise bi-allelic expression of IL-4. It remains unknown if biallelic cells represent a differently activated population, and if this population has 
specified roles in vivo, or if they are just cells which have initiated transcription from both IL-4 alleles by chance. Future work identifying extracellular markers of bi-allelic producing cells, or using multiple transgenic reporter systems will hopefully bring light to these questions.

\subsubsection{Transgenic reporter constructs are under similar regulation as parental alleles}

Reporter mice allow efficient identification, in real time, of cells expressing a protein of interest, however there are many caveats that have to been considered when interpreting data using these systems particularly if the protein is under allelic control. Many factors can affect the results garnered using these systems including; sensitivity of reporter detection, half life of the reporter, how insertion of the reporter has effected regulatory elements within the gene, and as seen with the huCD2 reporter mouse, the potential for non-specific transfer of reporter to other cells. However when these factors are considered and controlled for reporter mice can provide important information about the regulation of gene expression.

Genes like Il4 are under such strong regulation, with few cells expressing the protein and of those the majority only expressing from one allele, therefore using heterozygous knock-in reporter mice leads to decreased numbers of cells expressing WT IL-4, and thus decreased the amount of IL-4 available to drive Type 2 immune responses. It was hoped that transgenic reporter mice might allow reporting while maintaining WT expression of the parental alleles, and potentially marking every cell that was producing IL-4. Unfortunately I discovered that despite probably being located in a random site outside of the Th2 locus, the transgenic IL-4 reporter remains under allelic control (Figure 3.8), meaning that it's presence has a slight, non-significant effect on WT IL-4 production. More importantly only a subset of IL-4 producing Th2 cells were marked by reporter. This phenomenon was exploited to assess some of the properties of allelic regulation, specifically that bi-allelically expressing cells have an increased propensity to express from a third allele (Figure 3.9). From this I inferred that these cells have an excess of factors that allow bi-allelic expression. While the allelic expression by the transgenic reporter has had its uses it means that quantification of IL-4 reporting cells will likely significantly underestimate the true number of IL-4 producing Th2 cells, as many of these will be expressing of the WT IL-4 alleles rather than the transgenic reporter. Even with this limitation the 4C13R reporter mouse is still an effective tool for 
identifying which population of cells are expressing IL-4 or IL-13 and measuring proportional changes within these populations during Type 2 inflammation where close to WT cytokine is available to support the total response.

\subsubsection{Th2 IL-4 expression is independent of IL-4 signalling in vivo}

By using a transgenic reporter as an independent read out of IL-4 expression I was able to show that IL-4 expressing Th2 cells do not require IL-4 signalling for either their generation or later expansion within the LN (Figure 3.10). This further characterises IL4 as an effector cytokine of the Type 2 immune response rather than a differentiation driving cytokine as it has been thought of for decades. It also brings into question the role for LN basophils in the induction of Th2 immunity. The discovery that basophils can migrate to the reactive LN, express IL-4 and present antigen led to the theory that they may be what provides the initiating signal for Th2 differentiation ${ }^{377}$. As discussed in chapter 4 there are a range of conflicting publications showing that basophils are both important and not required for optimal Th2 responses. The limited role for IL-4 I have shown in this thesis somewhat diminishes their potential importance in generating Th2 responses. Both basophil numbers and their level of MHC II expression are significantly lower than B cells or DCs within the $\mathrm{LN}^{346}$, decreasing the chance of them presenting antigen to a cognate $\mathrm{CD} 4^{+} \mathrm{T}$ cell. While it is possible that basophil IL-4 may support early B cell responses, particularly as trending increases in basophils could be seen within the LN as early as day 4 of MC903 treatment, this link remains to be shown. Specific depletion experiments will provide light on the role of basophils in activating lymphoid cells within the LN.

IL-4 did not appear to directly affect Th2 cells, however it may be important in suppressing other $\mathrm{CD} 4^{+} \mathrm{T}$ cell responses. The increased proportion of IL-4 reporting cells in IL-4 WT mice indicated the absence of IL-4 allowed expansion of other Th subsets. This correlates with results observed using IL-4R $\alpha^{-/-}$mice where increased levels of T-bet mRNA are detected in the LN of IL-4 signalling deficient mice. The role of IL-4 in limiting the expansion of other $\mathrm{CD} 4^{+} \mathrm{T}$ cell subsets may be important in regulating downstream mixed or non-Type` 2 effector functions, as IL-4R $\alpha^{-/}$mice displayed increased mRNA for IgG2a isotype antibodies. ${ }^{83}$

IL-13 is not expressed within the LN of mice (Figure 4.8) so utilising the pTh2 assay did not provide any data on the effect of partial or complete IL-4 deficiency on the 
induction of $\mathrm{CD}^{+} \mathrm{T}$ cell IL-13 expression. IL-4 signalling increases the level of GATA3 expressed by the newly differentiated Th2 cells ${ }^{212}$, and with IL-13's reliance upon high GATA3 ${ }^{84}$ for expression it would be expected that IL-4 availability would support IL-13 expression. IL-13 function can be observed in IL-4 or IL-4R $\alpha$ deficient (genetically or antibody depleted) mice in models of allergic asthma ${ }^{355}$, pulmonary cryptococcosis $^{378}$ and intestinal helminth expulsion ${ }^{156}$, though these responses are diminished, indicating that while IL-4 is not required for IL-13 it may play a role in optimal expression. My finding in this thesis that IL-4 deficient mice were still protected from $\mathcal{N}$. brasiliensis infection in a IL-13 dependent manner also showed that sufficient IL13 expression to provide protection can be achieved in the absence of IL-4, though the source of this IL-13 was not assessed (Figure 6.3). Utilising the mice designed in this thesis with IL-13 reporting from a transgenic reporter construct crossed to IL-4 WT, heterozygous and deficient strains it will be possible to assess the role of IL-4 sufficiency to the expression of tissue IL-13 by CD4+ $\mathrm{T}$ cells.

\subsubsection{The expression of IL-4 and IL-13 is differentially regulated}

The 4C13R transgenic reporter mice allowed careful observation of the expression of IL-4 and IL-13 without the requirement of restimulation or intracellular staining, which led to the observation of consistently delayed expression of IL-13 after IL-4 (Figure 4.1). This along with the observations that APC stimulated $\mathrm{CD} 4^{+} \mathrm{T}$ cells in Th2 inducing conditions require multiple rounds of activation for IL-13 reporting, and the differential expression of IL-13 between LN and lung, led me to make several inferences on the regulation of IL-13 expression. There is little biological reason for IL-13 to be expressed within the mouse LN. The vast majority of cells within the mouse LN are T cells and B cells, which mostly have been shown to not express the IL-13R $\alpha$ I so cannot respond to IL-13 ${ }^{179}$. Other than lymphocytes there are resident and migratory DC populations as well as sub-capsular macrophages, cells that can express the IL-13R $\alpha 1$, but also the IL$4 \mathrm{R} \alpha$ and the $\gamma_{\mathrm{c}}$ chain $^{379,380}$, so would also be able to respond to the IL-4 expressed by Th2 and Tfh cells within the $\mathrm{LN}^{81}$. So even the few cells that could respond to IL-13 within the LN could just as likely respond to IL-4 via the higher affinity IL-4 receptor complex, so there appears to be little role for IL-13 within the murine LN.

Conservation of IL-13 expression by Th2 cells until within the peripheral tissue is consistent with IL-13 mediating its effects in the tissue, not the LN, but the mechanisms 
that eventually allow IL-13 expression are remain unclear. Multiple or sustained stimulations through the TCR in vitro are required (Figure 4.4 and Figure 4.6) and this may give hints to the in vivo situation as well. It is tempting to interpret this as indicative that Th2 cells receive a primary stimulation within the LN, enough to activate them and potentially turn on IL-4 expression; they then migrate to tissue sites where local APCs present antigen, providing a second restimulation and allowing IL-13 expression. Imaging studies refute the likelihood of this model though, with GD4 $\mathrm{T}$ cells demonstrated to have multiple interactions with DCs of varying durations within the LN over the first 3 days of stimulation ${ }^{381}$, so it is unlikely to be simply the number or length of stimulations that limits LN IL-13 expression.

The DC phenotype may also be very important at inducing IL-13 expression from Th2 activated $\mathrm{CD} 4{ }^{+} \mathrm{T}$ cells. In the in vitro model, I used splenic CD11c expressing cells from naïve mice as the APGs to both activate and restimulate at day 3. It is plausible that the DCs presenting antigen within the reactive LN are phenotypically different to APCs within the tissue or naïve $\mathrm{LN}^{382}$, and the differences in cytokine production and costimulatory molecule expression may limit LN DCs ability to induce IL-13 expression. As any potential IL-13 inducing signal that Th2 cells could receive from APGs within the tissue is probably absent in the enriched $\mathrm{CD} 4^{+} \mathrm{T}$ cell $\mathrm{Th} 2$ cultures, it is unlikely that tissue APGs are actively promoting IL-13 expression. The fact that the $\alpha \mathrm{CD} 3$ stimulation eventually induces IL-13 suggests that LN APGs, or something about the LN environment, may actively suppress IL-13 expression by the CD4 ${ }^{+} \mathrm{T}$ cells. This effect can be partially overcome with FTY720 treatment, which increases the proportion and number of IL-13 reporting $\mathrm{CD}^{+} \mathrm{T}$ cells in the $\mathrm{LN}$ but it remains to be found what is causing this increase of IL-13 reporting. Use of activated APCs or DCs from reactive $\mathrm{LN}$ in the in vitro model may indicate if it is a signal from the LN APC that is inhibiting IL-13 expression.

The delay in IL-13 expression may be a $\mathrm{CD}^{+} \mathrm{T}$ cell intrinsic timing mechanism that inhibits IL-13 until a sufficient amount of time has progressed. For this to be true cells must have a way to measure time. One available mechanism is counting cycles of cell division. It has been previously shown that $\mathrm{CD}^{+} \mathrm{T}$ cell production of IL-2, IL-4 and IFN- $\gamma$ expression is differentially regulated in terms of timing, which depends partially upon number of cell divisions ${ }^{383}$. Bird et al. demonstrated that cells stimulated without a polarising influence $(\alpha \mathrm{CD} 3+\alpha \mathrm{CD} 28+$ IL-2) IL-2 production could be detected after 
one day of activation before any proliferation of the cells; IFN- $\gamma$ could be detected on day 2 in both undivided and proliferated cells, and it took at least 3 days and 4 divisions for cells to express IL-4. The regulation was dependent upon epigenetic changes at the cytokine gene loci that occurred with successive proliferation cycles. Drugs that increased either histone acetylation or cytosine demethylation led to increased proportions of cytokine positive cells and production by less proliferated cells. ${ }^{383}$ However in the presence of Th2 polarising conditions IL-4 expression had been detected by unproliferated cells but it still required at least 48 hours of stimulation before IL-4 was detectable ${ }^{384}$ and our own unpublished data). To further complicate this, cells stimulated by antigen in the presence of IL-4 and L-mimosine, a drug that inhibits division by arresting cells in G1/S phase, could not express IL-4. Once Lmimosine was washed from the culture and cells were restimulated, they could express IL-4, even before they had progressed through a round of proliferation ${ }^{384}$. So while division isn't required, cell cycle inhibitors can inhibit IL-4 expression. It remains unknown if cell cycle inhibitors or treatments that increase acetylation, or demethylation would affect IL-13 expression. Experiments using reporters to accurately monitor cytokine and transcription factor expression along with dilution dyes and epigenetic analyses will shed further light on the elements which control expression of all the Type 2 cytokines.

In mice, where the majority of lymphocytes do not express the IL-13R $\alpha 1$ there is little requirement for IL-13 within the LN, however the expression of IL-13R $\alpha 1$ by B cells in humans means there may be species specific differences in the regulation of IL-13 expression $^{179}$. IL-13 was first recognised as a human B cell activating cytokine that induced IgE class switching comparable to IL-4162. Therefore there are two possibilities, either in humans $\mathrm{CD}^{+} \mathrm{T}$ cell IL-13 expression is present in the LN providing a redundancy with IL-4 for driving of IgE isotype switching, or IL-13 remains absent in human LNs as seen in the mouse models. $\mathrm{IgE}$ is a very potent antibody, able to mediate systemic effects at serum titres much lower than the other antibody isotypes ${ }^{19}$. The immune system carefully regulates $\mathrm{IgE}$ expression ${ }^{120}$, as inappropriate production of IgE with specificities against innocuous antigens can lead to potentially fatal anaphylactic reactions ${ }^{137}$. Due to this fact it is feasible that IL-13 expression may be excluded from the human LN even though B cells are able to respond to it. 
There is evidence available that human $\mathrm{CD}^{+} \mathrm{T}$ cells do not regulate IL-13 as mouse cells do. Restimulation of peripheral blood mononuclear cells from both adults and neonates induced rapid expression of both IL-4 and IL-13 mRNA, indicating that peripheral CD4 ${ }^{+} \mathrm{T}$ cells begin transcribing these cytokines simultaneously, though this doesn't mean they will translate both proteins together ${ }^{157}$. The Human Protein Atlas has measured some histological staining for IL-13 within the LN as well ${ }^{385}$. A clinical trial with the IL-13 blocking antibody has shown that IgE levels can be decreased by inhibition of IL-13 signalling ${ }^{386}$. The effects on IgE of IL-13 signalling measured in mice is considered to be indirect ${ }^{387}$, however as human B cells can respond to IL-13 the mechanisms via which IL-13 promotes IgE in vivo remain uncertain. It would be particularly interesting to assess if human Tfh cells expressed IL-13, as it has been found that IL-21, a cytokine produced by Tfh, potentiates human B cell responses to both IL4 and IL-13 and synergises with these cytokines to induce increased B cell proliferation ${ }^{388}$. In vitro studies assessing the time of protein production by stimulated naïve human $\mathrm{CD}^{+} \mathrm{T}$ cells should determine if IL-4 and IL-13 are as temporally separated by human CD4+ $\mathrm{T}$ cells as they are in mouse.

In some situations there is significant overlap of IL-4 and IL-13 function, as observed in the case of lung protection against $\mathcal{N}$. brasiliensis infection (Figure 6.3) however in other models IL-13 has been shown to have a critical non-redundant role ${ }^{188}$. The discoveries in this study that IL-13 is so differently regulated from IL-4 indicate that there are unrecognised pathways that distinctly control the expression of the Type 2 cytokines. Cytokines consistently co-expressed are not necessarily controlled via the same mechanisms, and careful characterisation of these mechanisms will lead to identification of specific targets to modulate individual cytokines as required.

\subsubsection{Innate immune cells associated with Type 2 display differential cytokine regulation}

This thesis investigated several types of innate immune cells and how they were responding to environmental cues that mediated their migration and expression of cytokines. Since this project a study has been published describing high levels of TSLP induced by MC903 treatment drew basophils from the blood into the site of treatment and the draining $\mathrm{LN}^{339}$. Kim et al. have reported a difference in the numbers of basophils entering the skin of TSLP-/- mice, which for an unknown reason I did not replicate in this study (Figure 5.12). However I did not observed systemic basophilia, in 
fact basophil numbers and proportions in blood actually decreased in treated animals (Figure 5.10), assumedly due to migration from the circulation to the treated tissues and LN. TSLP is able to promote haematopoiesis of basophils, however this was shown in models where recombinant TSLP was administered or transgenic production was induced $^{329}$. It may be that in natural TSLP inducing inflammation models there are two distinct signals, one that induces basophil haematopoiesis and drives systemic basophilia, and another, potentially TSLP, which mitigates basophil entry into tissue and LNs. This could explain why increased circulating basophils have been reported in $\mathcal{N}$. brasiliensis infected TSLPR ${ }^{-/-}$mice compared with infected WT ${ }^{389}$. If decreased basophil numbers were observed in the lungs and LN of the TSLPR ${ }^{-1}$ mice this would argue strongly for TSLP generally working as a chemotactic agent. So MC903 treatment, with it's localised impact, draws basophils to the draining LN via TSLP but apparently lacks the systemic signals present during helminth infection that promote haematopoiesis and systemic basophilia. The haematopoiesis-inducing signal absent from MC903 treatment may be IL-3. Giacomin et al. found antibody neutralisation of neither IL-3 nor TSLP alone caused any changes to circulating blood basophil number during Trichinella spiralis infection. $\alpha$ TSLP treatment of IL-3R deficient mice however prevented any increase in blood basophils. Furthermore $\alpha$ TSLP decreased the numbers of splenic and mesenteric LN basophils during Trichinella spiralis infection, while $\alpha$ IL-3 only caused a modest decrease in the number that arrived in the LN. ${ }^{341}$ While the role of TSLP, and TSLP elicited basophils, remain to be elucidated many of the cytokine deficient, and cell depletion tools are now available, so it is likely that this field will progress quickly over the next few years.

I found that dILCs in the skin did to not show strong responses in any of the models of inflammation used in this paper, not even direct injection of the cytokine IL-33 or TSLP caused proliferation or changes in IL-13 expression. This is in particular conflict with two recent papers that have measured ILC responses using the MC903 model 338 , 339. The first measured ILC responses but mostly in the draining LN and found they did expand in a TSLP dependent fashion. It was difficult to draw direct comparisons between the observations in this thesis because the paper measured LN ILC responses ${ }^{338}$. The second paper also using the MC903 paper measure dILC expansion within the skin between days 4 and 7 of treatment and found that IL- 4 produced by the basophils was responsible for the ILC expansion. Adoptive transfer of TSLP elicited 
basophils from WT, but not IL-4 ${ }^{-/-}$donors were sufficient to induce atopic dermatitis and cause ILC expansion ${ }^{339}$. Why I did not replicate the observations made by these studies in this thesis is unknown. It seems unlikely that slight variations in MC903 concentration would account for such differences in cell specific responses. This publication does seem to agree with my observation that ILCs do not directly respond to TSLP; Kim et al. demonstrated that it is TSLP activation of basophil IL-4 that modulates the ILG responses ${ }^{339}$. Similar to IL-2, TSLP signals through STAT5, and STAT5 has important roles in inducing IL-4 expression in CD4 ${ }^{+} \mathrm{T}$ cells. By expressing the TSLPR, along with calcium signalling pathway such as crosslinking of FceRI bound IgE, basophils may use these signalling pathways as triggers for IL-4 production.

The observations within this thesis that ILC populations within the skin are resistant to depletion and that lung ILC2 depletion can only be partially achieved is in disagreement with many publications. Various studies have reported the ability to use antibodies of various concentrations to deplete populations of ILCs from the skin draining LN, ${ }^{338,390}$ lung $^{240}$ and intestines ${ }^{248}$. One difficulty is that many of these studies use antibodies against Thyl ( $\alpha$ CD90 antibodies) or PC61 ( $\alpha$ CD25), but continued to use CD90 or CD25 expression as a gating criterion for ILC identification2236, 240, 274, 338 . I found almost total CD90 masking in the lung and spleen (though not in the skin indicating restricted antibody access to the dermis), so careful analysis of ILC expressed molecules including CD25, KLRG-1 and CD127 were utilised to assess depletion, at best an approximate 50\% reduction in ILC number was achieved in inflamed lungs (Figure 8.3). This was sufficient to see decreased efficacy in the IL-2c driven protection in Rag1\%- mice (Figure 6.9), and may explain why other publications can measure ILC dependent effects, despite insufficient depletion checks being performed. Why these cells are so resistant to depletion in unknown. It is possible they rarely circulate, the circulation and migration kinetics of ILGs have not been studied. If they were resident cells then the local immune cell populations would be relied upon to mediate antibody dependent cell-mediated cytotoxicity. Antibody-bound circulating populations can be picked up as they pass through the liver or spleen ${ }^{391}$. If there were a lack of appropriate cells, such as antibody responsive macrophages or NK cells within the tissue could mean that resident cells are not very likely to be depleted in situ and are relatively protected from antibody depletion. Using tools that allow tracking of cells, like the photosswitching reporters ${ }^{392}$ will allow investigation into the migratory capabilities of ILCs and other depletion resistant populations. 
ILC2s of the lung, unlike dILCs, rapidly responded to IL-33 treatment increasing their expression of IL-13 within 24 hours (Figure 6.7). ILGs are proposed to act as damage sensors and rapidly respond by production of proinflammatory cytokines IL-5 and IL13, and also damage control proteins such as amphiregulin, which mediate epithelial healing ${ }^{393}$. IL-33 is not the only factor known to stimulate ILC2 responses. IL-25 has also been implicated ${ }^{394}, 395$, and a recent publication identified prostaglandin D2 as a potent activator of ILC2s within the lung ${ }^{396}$. ILC2s within the lungs play dual roles; promoting inflammatory responses against invading parasites and in AHR models ${ }^{397}$, and through their support of M2 macrophages and epithelial healing via amphiregulin they also are critical to healing damaged and maintaining tissue function ${ }^{240}$. Why dILCs did not respond to IL-33 remains to be investigated as, albeit a small percentage, some of the dILC population were detected expressing the IL-33R (ST2) (Figure 5.2). It is possible that the dILCs represent a quiescent ILC population that respond to stimulation from other members of the immune system such as CD4 ${ }^{+} \mathrm{T}$ cells and basophils, and this study did not accurately assess their function at the correct time points to observe their responses.

This thesis has identified several signalling pathways that mediate innate cell responses to varied inflammatory stimuli. These pathways are critical to the early functions of the Type 2 immune response and may play important roles in how the larger response will form over time. Understanding these early innate responses are just as important as characterising the later adaptive immune cell functions.

\subsection{6 $\mathrm{CD}^{+} \mathrm{T}$ cells support ILC2 responses}

One of the major findings of this thesis is that $\mathrm{CD} 4^{+} \mathrm{T}$ cells provide signals that support the maintenance of ILC2s within the lung after $\mathcal{N}$. brasiliensis infection (Figure 6.6). The exact mechanisms that GD4+ $\mathrm{T}$ cells employ to communicate with ILCs are unclear but there are several candidates. In this thesis and related work, IL-2c was used to stimulate both skin and lung ILC responses to great effect. ILC2s responding to low dose IL-2 treatments used in clinical trials to treat autoimmune diseases have been implicated in inducing systemic eosinophilia via IL-5 ${ }^{398}$. Systemic IL-2c treatment leads to a strong proliferative and cytokine response from ILC2s and while it provides a useful model for measuring the capabilities of stimulated ILC2s it is likely unrepresentative of the true stimulatory signals ILC2s are receiving in vivo. IL-2 is only transiently expressed by most activated $\mathrm{T}$ cells, and though Th1 differentiated CD4 ${ }^{+} \mathrm{T}$ cells maintain IL-2 expression 
longer than other $\mathrm{T}$ cell subsets ${ }^{399}$, Thl cells are a rare population in $\mathcal{N}$. brasiliensis infection ${ }^{84}$. In a natural setting ILC2s will likely be exposed to limited IL-2 for short times but they will be exposed to other activating stimuli. Similar to skin ILCs, ILC2s within the lung have been shown to respond to basophil derived IL- $4{ }^{400}$, . It is therefore feasible that ILC2s could respond to both basophil and Th2 derived IL-4, especially considering the relatively high numbers of IL-4 reporting $\mathrm{CD} 4^{+} \mathrm{T}$ cells during $\mathcal{N}$. brasiliensis infection (Figure 4.7). IL-9 has also been shown to be a potent survival factor for ILC2 and required to maintain their numbers after $\mathcal{N}$. brasiliensis infection. Using fate reporters (which mark every surviving cell that has at any point expressed IL-9) it was found the majority $(>80 \%)$ of reporter positive cells were ILC2s, the remainder being $\mathrm{CD}^{+}{ }^{+} \mathrm{T}$ cells $^{74}$. This indicates that survival-promoting IL-9 may be working in an autocrine fashion. However fate reporters do not allow identification of cytokine producing cells in real time, and it was unknown which populations maintained their production of IL-9 long term. There is also the potential for direct communication between ILC2s and CD4 ${ }^{+} \mathrm{T}$ cells, expression of MHC II on the ILC2s can facilitate cell-cell interactions in vitro ${ }^{358}$, so it remains feasible that these exchanges could occur in vivo. These interactions could promote communication via other receptors, providing pro-survival and activation signals to the ILC2s.

$\mathrm{CD}^{+} \mathrm{T}$ cells are required to maintain ILC2 responses in a secondary infection, though I have shown that cytokine stimulated ILC2s were able to generate protective immune responses in the absence of $\mathrm{CD}^{+}{ }^{+} \mathrm{T}$ cells (Figure 6.8, Figure 6.9 and Figure 6.10). Early cytokine expression detected using the 4G13R transgenic reporter found that ILC $2 \mathrm{~s}$ were responding within 24 hours of infection, indicating that even to natural stimuli ILC2s show rapid activation. The question remains why these early responding ILC2s cannot provide protection against a primary infection. It is likely that while rapid, the larvae are still faster, exiting the lungs within 3 days of infection, not allowing enough time for the ILC2 produced IL-13 to switch on the anti-helminthic programming of a sufficient number of macrophages and other effector cells. It is also possible that, as is often an issue raised when regarding the relative importance of ILCs, there may simply be too few to respond sufficiently in a primary infection. I found the numbers of ILC2s are raised up to 30 days post infection and further expanded two days after a secondary infection in a $\mathrm{CD}^{+} \mathrm{T}$ cell dependent manner. The $\mathrm{CD}^{+} \mathrm{T}$ cell independent protection model required cytokine treatment two days prior to infection, effectively giving the ILC2s a head start to expand and drive anti-helminthic responses to prepare 
the protective environment within the lung. Similarly, IL-2c treatment of CD4 ${ }^{+} \mathrm{T}$ cell depleted mice prior to secondary infection increased ILC2 number as well as cytokine production proving enough signal to maintain the protective immune responses generated during the primary infection.

To date many of the roles of ILGs have been demonstrated in T cell independent models. In this thesis I have found that stimulated ILC2s are sufficient for both priming, and maintaining protective responses within the lung against $\mathcal{N}$. brasiliensis. Due to their overlapping functions ILC2s and Th2 cells are often thought to be redundant, this study has found that both populations constitute significant proportions of the IL-13 producing cells, and as seen with $\mathrm{CD}^{+} \mathrm{T}$ cell depletion, it is possible ILC2 depletion would also lead to decreases in the protective response. This thesis has lacked tools for specific targeting of ILC2s to determine their relative contributions to protective responses in a T cell sufficient host. Using such tools will elucidate if the early responses by ILC2s, and their contributions to cytokine production during a secondary infection are important at developing the overall immune responses.

\subsection{7 $\mathrm{CD}^{+} \mathrm{T}$ cells are the central mediator of Type 2 immunity}

Long term depletion of $\mathrm{CD}^{+} \mathrm{T}$ cells led to decreased protective responses against a secondary infection, however we have shown the presence of $\mathrm{CD}^{+} \mathrm{T}$ cells was not required during the protective immune response against $\mathcal{N}$. brasiliensis (Figure 6.5). That $\mathrm{CD}^{+}{ }^{+} \mathrm{T}$ cells aren't required to directly effect the larval survival isn't surprising; CD4 ${ }^{+}$ $\mathrm{T}$ cells have not been reported to produce anything that could directly harm the larvae. The fact that they can be absent, and yet immune responses remain effective indicates a much less direct role for $\mathrm{CD}^{+} \mathrm{T}$ cells than was previously thought. Work done alongside of this thesis has shown that M2 macrophages are key effector cells that provide protection, and it is the phenotype of these cells that $\mathrm{CD} 4{ }^{+} \mathrm{T}$ cells maintain through production of IL-4 and IL-13354. Previous studies have shown that M2 macrophages require constant STAT6 dependent signalling to maintain their phenotype $^{401}$, The loss of protection when $\mathrm{CD}^{+} \mathrm{T}$ cells are depleted for a week correlates with this. However the source of IL-4 or IL-13 in the short-term absence of $\mathrm{CD}^{+} \mathrm{T}$ cells is unknown. It is possible that ILC2s are not as sensitive to the loss of the support provided by CD4+ $\mathrm{T}$ cells, and are able to provide sufficient IL-13 to maintain M2 macrophages in the short term. Through this work we have confirmed that CD4 ${ }^{+} \mathrm{T}$ cells are critical to maintaining Type 2 immunity within the lung, and that these 
responses can only be sustained for a short time in the absence of these central mediators.

It has been shown using FTY720 to inhibit migration of CD4 ${ }^{+} \mathrm{T}$ cells from the LN to the lung, that lung resident populations of $\mathrm{CD}^{+} \mathrm{T}$ cells are able to respond to a primary infection and provide protection against a secondary infection ${ }^{134}$. The fact that tissue resident $\mathrm{CD}^{+} \mathrm{T}$ cells show a greater propensity for IL-13 expression that $\mathrm{LN}$ cells, and that inhibiting LN migration has no effect on the number for IL-13 expressing cells (Figure 4.11), indicates that these cells may account for the majority if not all CD4+ $\mathrm{T}$ cell derived IL-13 within the lung. In vitro stimulation found no preference for lung cells to express IL-13 compared with spleen derived CD4 ${ }^{+} \mathrm{T}$ cells (Figure 4.9), so it remains unknown what control IL-13 expression in vivo and if these lung resident cells represent a different population of $\mathrm{CD}^{+} \mathrm{T}$ cells primed to express IL-13 without needing activation in the $\mathrm{LN}$. While it is known $\mathrm{CD}^{+} \mathrm{T}$ cells are critical to mediating Type 2 immunity, much about how they are activated, their kinetics of migration during inflammation and their regulation of effector function remain to be discovered. 


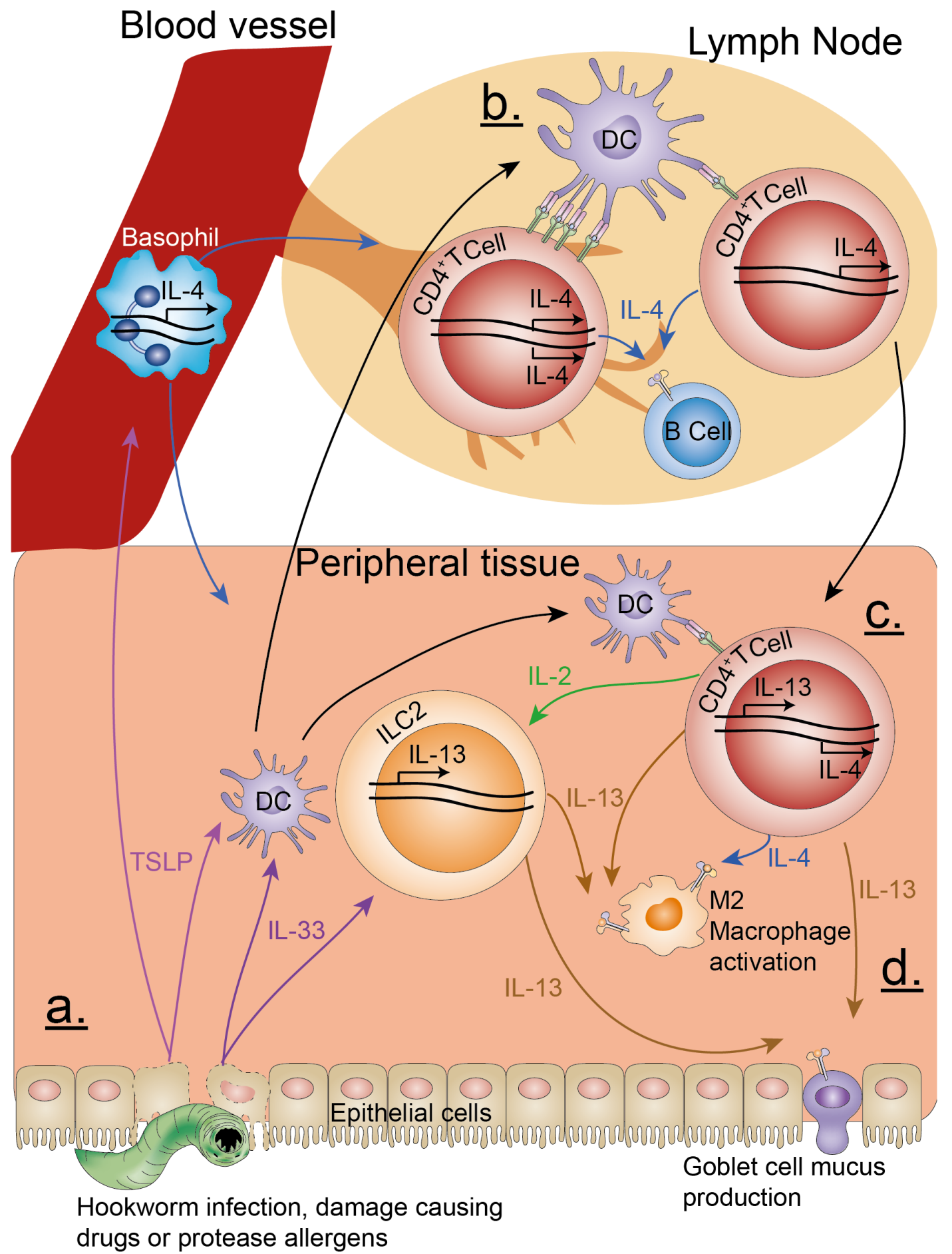

Figure 7.1 - Summary of discoveries from this thesis in the greater context of Type 2 immune responses.

a. Damaged epithelial cells release cytokines including TSLP and IL-33. TSLP acts systemically to induce IL-4 expression from basophils and migration of these basophils to the LN and tissue. IL-33 activates tissue resident ILC2s, which rapidly begin producing IL-13. DGs responding to epithelial derived cytokines become activated and migrate to the LN. b. Antigen loaded DCs present to $\mathrm{CD}^{+} \mathrm{T}$ cells in the $\mathrm{LN}$ and induce IL-4 independent Th2 differentiation. Increased levels of TCR engagement may increase the chances of a CD4 ${ }^{+} \mathrm{T}$ cell expressing IL-4 biallelically as seen in vitro. B cells are the predominant IL-4 responding cell type within the LN. c. Several days after the $\mathrm{LN} \mathrm{CD} 4^{+} \mathrm{T}$ cells begin expressing IL-4 tissue resident $\mathrm{CD}^{+} \mathrm{T}$ cell begin producing IL-13. FTY720 experiments indicate that most IL-13 expressing CD4+ ${ }^{+}$cells do not 
migrate from the LN at all, suggesting in situ activation by DCs and other tissue resident APCs. d. IL-4 and IL-13 drive M2 activation of macrophages, and IL-13 responsive goblet cells increase their production of mucus. In the context of a helminth infection, maintenance of the alternately activated macrophages by CD4+ $\mathrm{T}$ cell and ILC2 derived IL-4 and IL-1 3 provides protection against subsequent infections. CD4 ${ }^{+} \mathrm{T}$ cells also support ILC2 responses potentially through the production of IL-2. Adapted from Allen and Maizels, 2011.

\subsubsection{Key Findings}

- The expression of IL-4 and IL-13 are differently regulated by CD4+ ${ }^{+}$cells and by Type 2 associated innate immune cells

- The proportion of $\mathrm{CD}^{+} \mathrm{T}$ cells expressing IL-4 bi-allelically can be altered by modifying the level of TCR stimulation

- IL-4 does not effect the generation or expansion of IL-4 producing CD4+ $\mathrm{T}$ cells in vivo

- Expression of IL-13 by in vitro differentiated $\mathrm{CD}^{+} \mathrm{T}$ cells requires multiple TCR ligations and is consistently delayed compared with IL-4

- Dermal innate lymphoid cells constitutively express IL-13, and skin migrating basophils in a model of atopic dermatitis express IL-4 in a TSLP dependent manner

- When stimulated with exogenous cytokines group 2 innate lymphoid cells in the lung can promote and maintain $\mathrm{CD}^{+} \mathrm{T}$ cell independent protective immune responses against Nippostrongylus brasiliensis infection 


\subsection{Conclusions}

The principle aim of my thesis was to compare the regulatory mechanisms that control IL-4 and IL-13 expression. By demonstrating the differential expression of IL-4 and IL13 both in vivo and in vitro I have found that mouse Th2 cells carefully regulate the timing and the location of their cytokine production to tissue sites where the cytokine will be most effective. Both IL-4 and IL13 demonstrate redundancy in their influences on anti-helminthic immune responses, and though $\mathrm{CD}^{+} \mathrm{T}$ cells are large producers, cells of the innate immune system produce significant amounts as well. Basophils and ILC2s contribute to Type 2 cytokine production but show differential preferences in which cytokine they produce. Basophils generally produce IL-4 and ILC2s express IL13, this demonstrates that different populations can selectively regulate which cytokine they preferentially express. Innate lymphoid cells, although sharing much in common with $\mathrm{CD} 4^{+} \mathrm{T}$ cells are a distinct effector population, responding quickly to infection, and when sufficiently stimulated capable of influencing the greater Type 2 immune response though production of IL-13. CD $4^{+} \mathrm{T}$ cells control many facets of the Type 2 immune response and their long-term depletion leads to significant abrogation of responses. Overall these data demonstrate significant regulation controlling the expression of IL-4 and IL-13, both within the CD4 ${ }^{+} \mathrm{T}$ cell population and between different innate cell populations. Despite some overlap in their functions, in some cases complete redundancy, the spatial and temporal regulation of IL-4 and IL-13 expression may allow the immune system to control cytokine specific effects during Type 2 immune responses. Understanding these mechanisms will allow the development of treatments that can specifically target the expression of individual cytokines, either boosting their effects as may be desirable with an anti-hookworm vaccines, or inhibiting them to limit pathologies associated with atopic diseases. 


\subsection{Future Directions}

This study has assessed many aspects of IL-4 and IL-13 expression and function, however further experiments need to be carried out to precisely define the mechanisms that control the expression of these two cytokines. Below I have described some experiments that could be carried out to further advance the understanding of Type 2 cytokine expression.

To assess further the mechanisms that control allelic regulation of the IL-4 allele, indepth analysis of intracellular signalling pathways is required. The first target should be to measure the relative levels of GATA3 within mono- and bi-allelically expressing cells as such strong evidence exists that GATA3 facilitates Il4 gene transcription. If a link is found then chromatin immunoprecipitation (ChIP) followed by high-throughput DNA sequencing (ChIP-seq) could be utilised to assess which regions of the Th2 locus GATA3 is binding and if there are differences between the differentially expressing Th2 populations. Unfortunately attributing functions to mono- and bi-allelically expressing cells is extremely difficult. In absence of a cell surface molecule that is differentially expressed, reporters that disrupt the expression of IL-4 are required to identify the mono- and bi allelic cells, and the decrease in available IL-4 may mask the actual functions these cells mediate. Transcriptome analysis may enable identification of molecules that could be used to delineate the differently expressing cells, and would allow functional studies within IL-4 sufficient cells.

Utilising the 4G13R reporters crossed to IL-4 deficient mice (4C13RxGFP/IL-4 and 4C13RxGFP/GFP strains) and looking at DS-red expression by tissue CD4+ $\mathrm{T}$ cells will allow assessment of IL-4 signalling on the expression of IL-13. Identification of GFP ${ }^{+}$ AmCyan $^{+} \mathrm{CD}^{+} \mathrm{T}$ cells will also allow correlation between bi-allelism and IL-13 expression. Finally the 4C13RxGFP/GFP mice can be used to assess ILC2 responses in the absence of IL-4, to assess if IL-4 is a factor that supports ILC2 responses.

Measuring GATA3 levels in vitro will also be important in understanding the delay in the expression of IL-13. Bcl-6 expression has been proposed as inhibitory for GATA3 expression so inhibitors of Bcl-6 might instruct whether it is functioning to limit GATA3 expression within our in vitro model. Potentially using treatments that drive global hyper-acetylation, or demethylation may also indicate if epigenetic factors are restricting IL-13 expression. Use of dilution dyes to measure proliferative cycles, and cell cycle inhibitors will assess if there is a requirement for proliferation that permits IL- 
13 production. The use of splenic DCs may also be promoting IL-13 expression, in vitro presentation by DCs from inflamed LNs will determine if the DC activation state plays a role in inducing IL-13 from the $\mathrm{CD}^{+} \mathrm{T}$ cells. A model is also available where transgenic $\mathrm{CD}^{+} \mathrm{T}$ cells are stimulated with 2 peptides of differing affinity. Using this model in vitro with APC driven Th2 activation will allow testing of how antigen affinity, antigen dose, DC activation state and accessory cytokines influence IL-13 induction.

Several models allowing in vivo depletion of ILC2s have been developed; using these mice will elucidate the exact contributions of ILC2s in activating M2 macrophages and protection against $\mathcal{N}$. brasiliensis infection. Adoptive transfer experiments of various gene knock-out $\mathrm{CD} 4^{+} \mathrm{T}$ cells may instruct via which signals $\mathrm{CD} 4^{+} \mathrm{T}$ cells use to sustain ILC2 responses during Type immune responses. Finally using TSLP and IL-3 deficient mice will provide information on the roles of these cytokines in the haematopoiesis and tissue migration of basophils 


\section{Appendix}

\subsection{Supplementary Data}

\subsection{1 $\mathrm{CD}^{+} \mathrm{T}$ cell gating scheme}

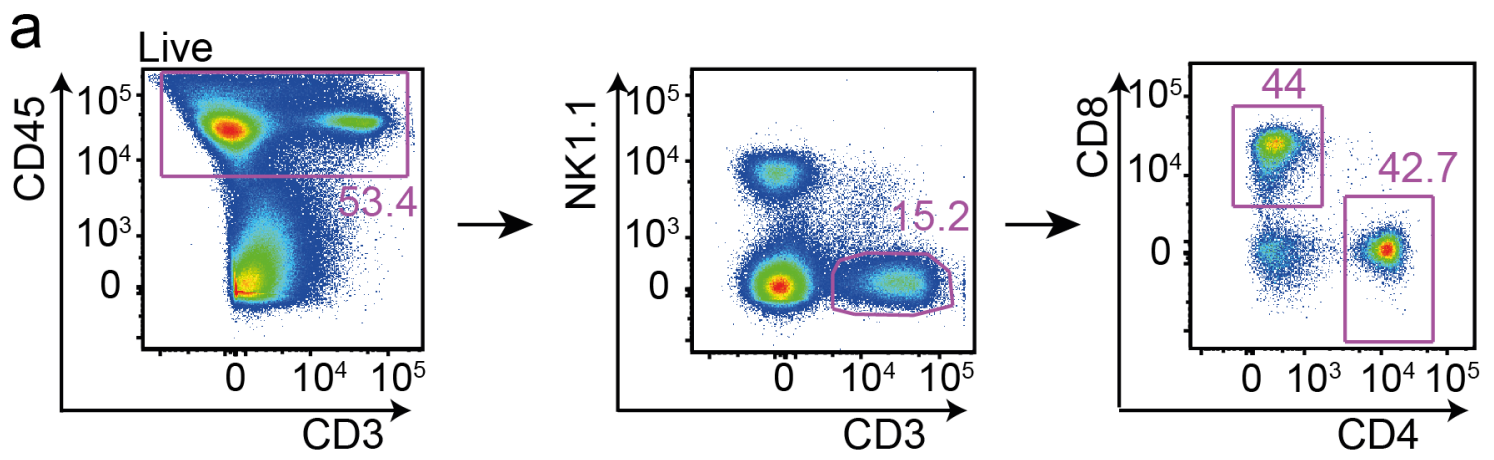

Figure 8.1 - Panel for the identification of $\mathrm{CD4}^{+} \mathrm{T}$ cells.

a. $\mathrm{CD}^{+} \mathrm{T}$ cells are selected by Live $\mathrm{NK} 1.1^{-} \mathrm{CD} 3^{+} \mathrm{CD} 8-\mathrm{CD} 4^{+}$. In some experiments $\mathrm{CD} 19$ was used to exclude B cells. 


\subsubsection{Cell Depletions}

\subsubsection{Depletion of $\mathrm{CD}^{+} \mathrm{T}$ cells from the lung}
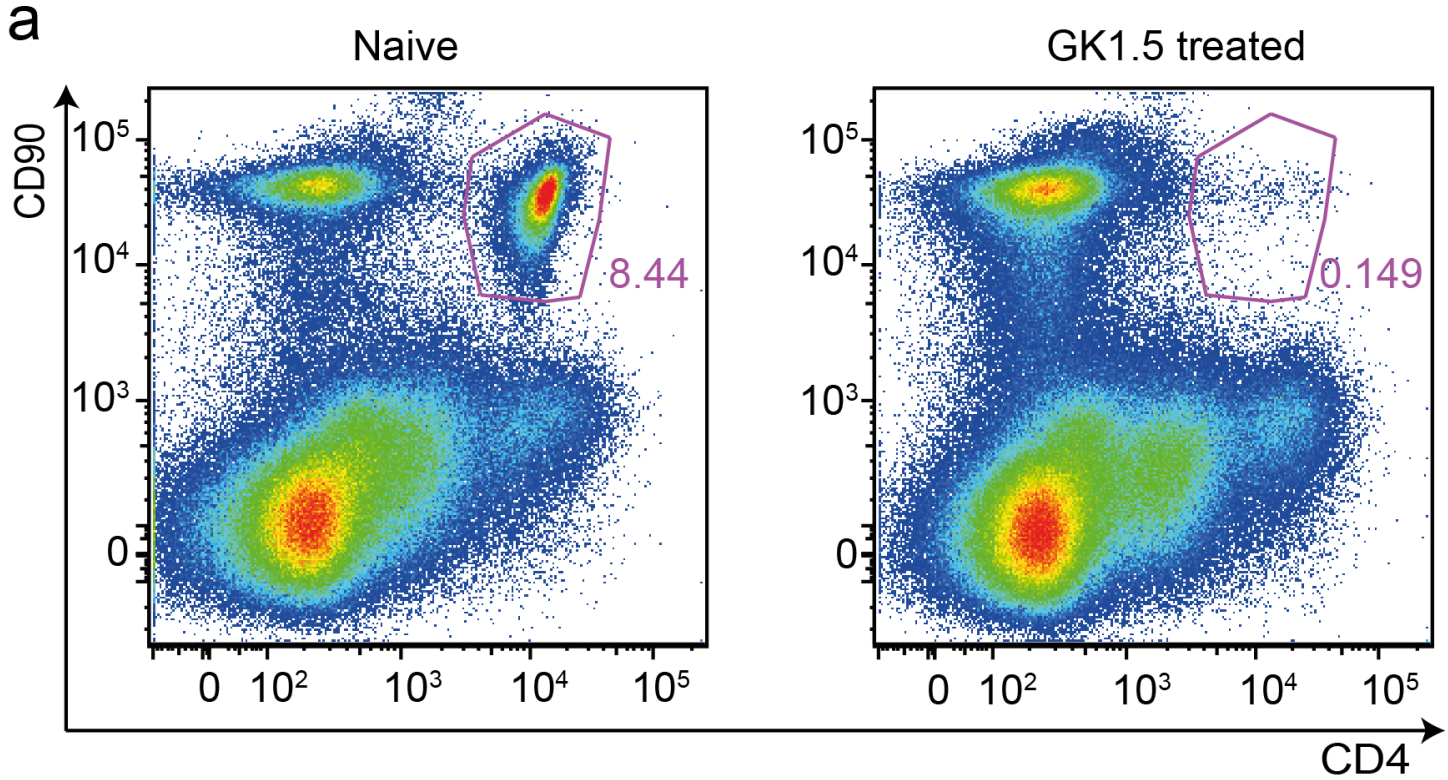

Figure $8.2-\mathrm{CD4}^{+} \mathrm{T}$ cell depletion in the lung is maintained for 4 days around infection.

a. Mice were treated with $0.5 \mathrm{mg}$ of antibody two days prior to $\mathcal{N}$. brasiliensis infection then the $\mathrm{CD}^{+} \mathrm{T}$ cell population within the lung were assessed by flow cytometry two days post infection. Lung cells were stained with a cocktail including the RM4-4 $\alpha$ CD4 clone specific for a separate epitope than GK 1.5. Plots are representative of many independent experiments. 


\subsubsection{Depletion of ILC2s from the lung}
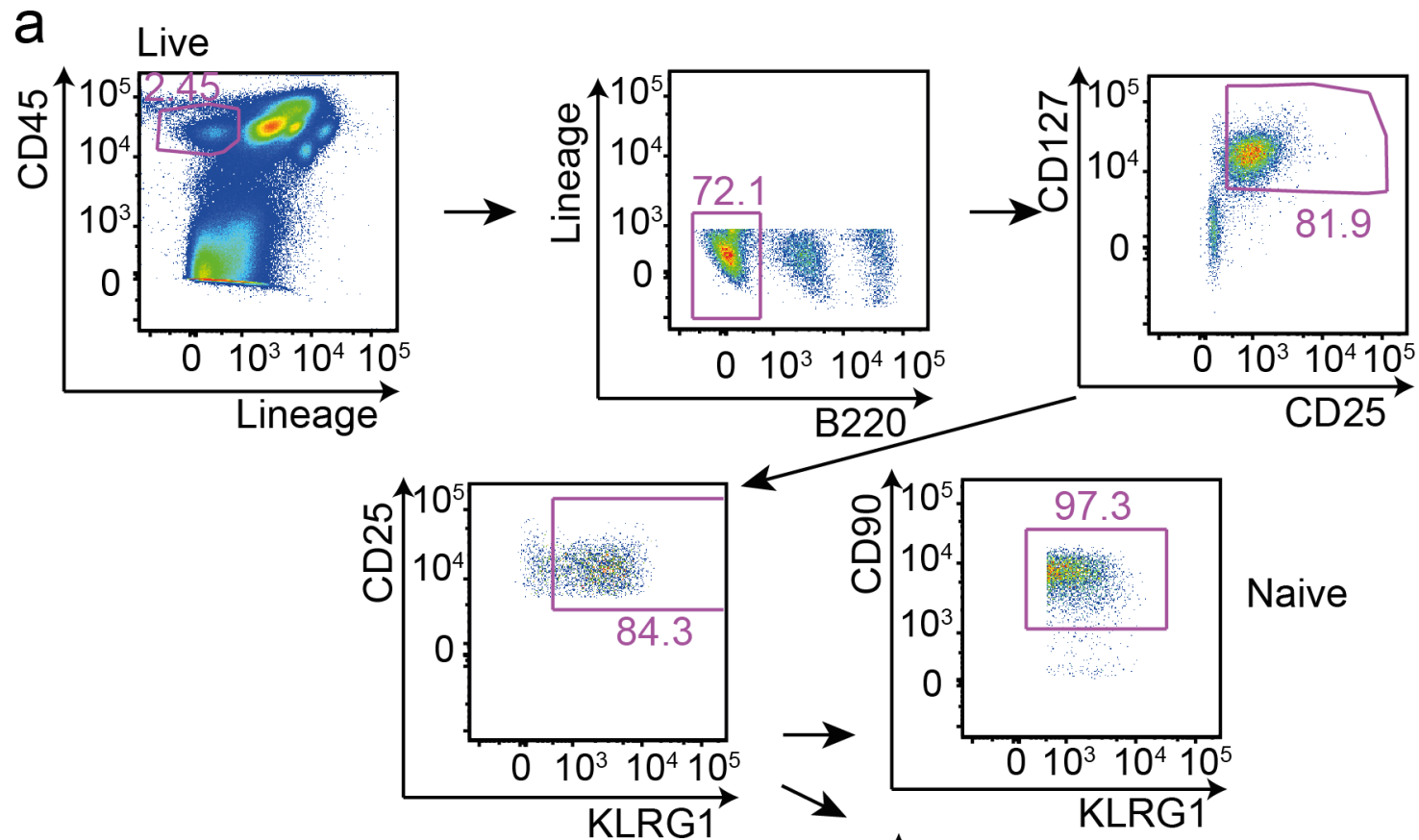

b

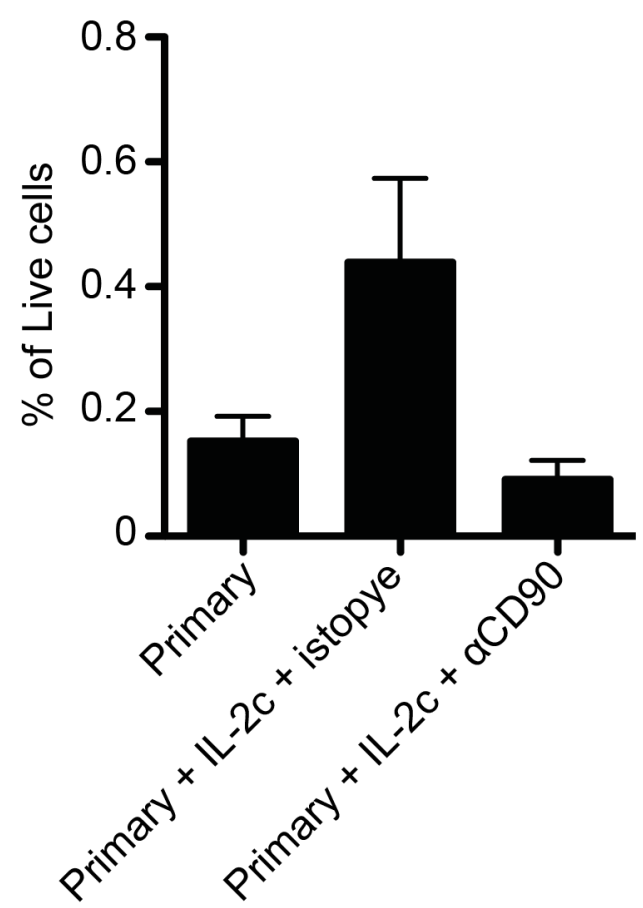

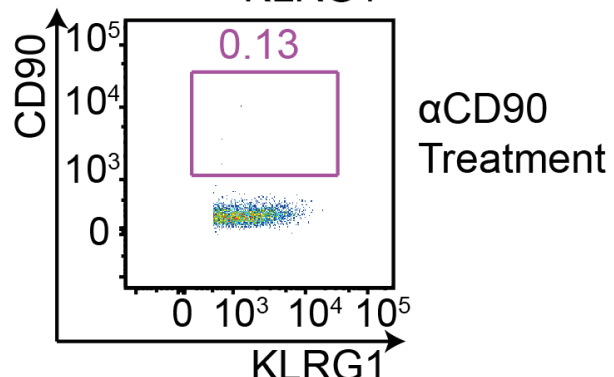

Figure 8.3 - Panel for assessing lung ILG2 depletion with $\alpha$ GD90 antibody.

a. In mice treated with $\alpha \mathrm{CD} 90$, depletion of lung ILC2s was assessed by comparing the numbers of Live CD45+ Lineage- B220- $\mathrm{CD} 127^{+} \mathrm{CD} 25^{+} \mathrm{KLRG} 1^{+}$undepleted mice. Mice treated with $\alpha \mathrm{CD} 90$ have complete masking of CD90 staining on their ILC2s. Plots are representative of many independent experiments. b. Percentage of live cells that are ILC2s in the lung during primary infection with and without IL-2c treatment and $\alpha$ CD90 treatment as per Figure 6.9. 


\subsection{Flow cytometer configurations}

\begin{tabular}{|c|c|c|c|}
\hline Gytometer & Laser & Filter set & $\begin{array}{l}\text { Fluorophore (bold denotes used } \\
\text { in this thesis) }\end{array}$ \\
\hline \multirow[t]{9}{*}{$\begin{array}{l}\text { BD LSRII- } \\
\text { SORP }\end{array}$} & \multirow[t]{3}{*}{ UV 355nm } & UV 740/35A & $\begin{array}{l}\text { BUV737 } \\
\text { BUV740 }\end{array}$ \\
\hline & & UV 450/50B & $\begin{array}{l}\text { AlexaFluor350 } \\
\text { DAPI } \\
\text { Hoescht Blue } \\
\text { Dyecycle Violet } \\
\text { LIVE/DEAD Fixable Blue }\end{array}$ \\
\hline & & UV 395/28G & BUV395 \\
\hline & \multirow[t]{4}{*}{ Violet 450nm } & V 780/60A & $\begin{array}{l}\text { Brilliant Violet } 786 \\
\text { QDot } 800\end{array}$ \\
\hline & & V 720/40B & $\begin{array}{l}\text { Brilliant Violet } 711 \\
\text { QDot705 }\end{array}$ \\
\hline & & V 660/20C & $\begin{array}{l}\text { Brilliant Violet } 650 \\
\text { QDot } 655 \\
\text { eFluor } 650 \mathrm{nc}\end{array}$ \\
\hline & & V 605/40D & $\begin{array}{l}\text { Brilliant Violet } 605 \\
\text { QDot585 } \\
\text { QDot605 } \\
\text { QDot625 } \\
\text { eFluor605 }\end{array}$ \\
\hline & & V 560/40E & $\begin{array}{l}\text { Pacific Orange } \\
\text { AlexaFluor } 430 \\
\text { QDot565 } \\
\text { Brilliant Violet } 570 \\
\text { Krome Orange } \\
\text { Lucifer Yellow } \\
\text { QDot545 }\end{array}$ \\
\hline & & V 525/50F & $\begin{array}{l}\text { Brilliant Violet } 510 \\
\text { AmCyan }\end{array}$ \\
\hline
\end{tabular}




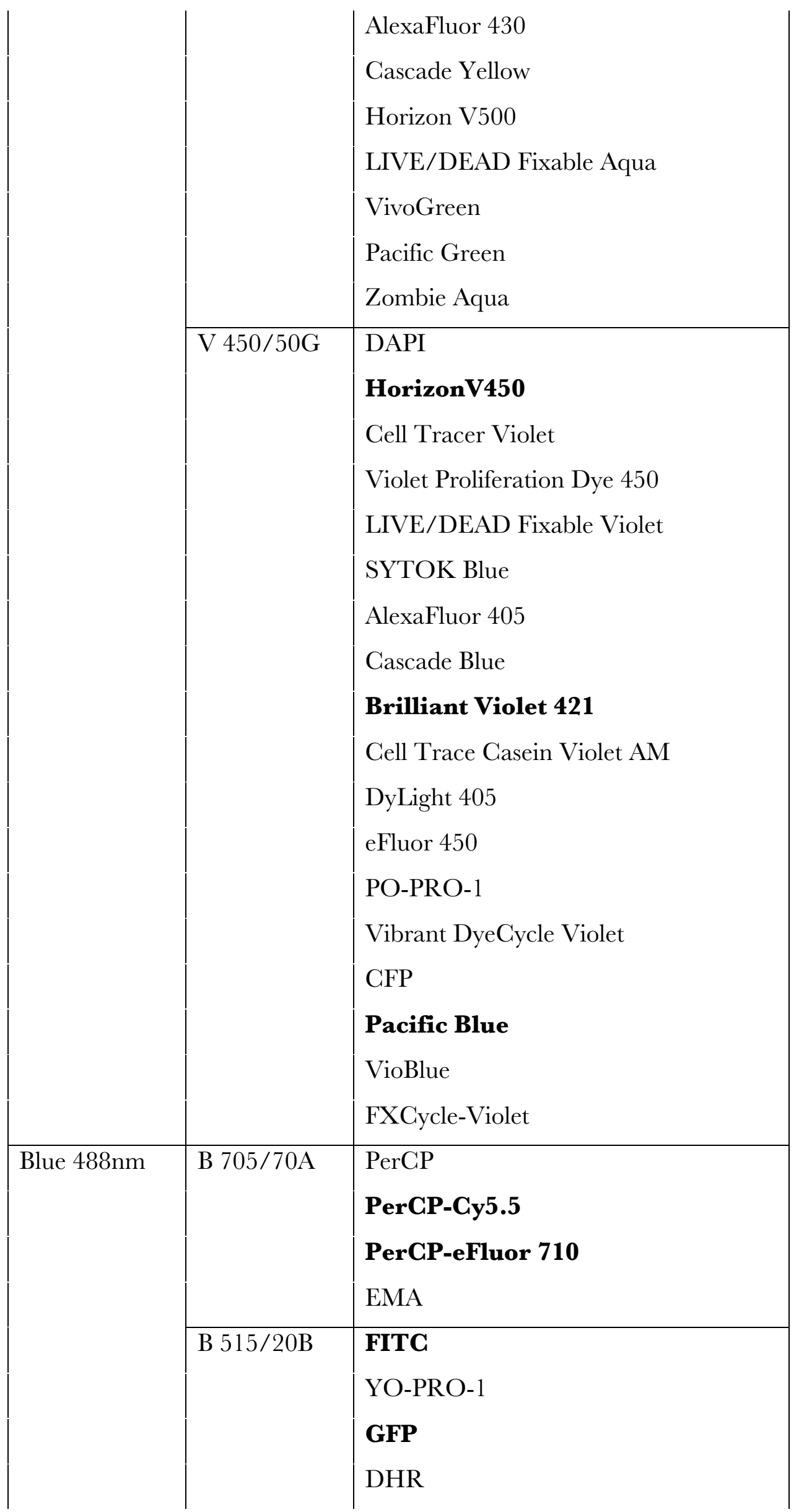




\begin{tabular}{|c|c|c|}
\hline & & $\begin{array}{l}\text { AlexaFluor } 488 \\
\text { AlexaFluor } 500 \\
\text { Cell Trace Green AM } \\
\text { CFSE } \\
\text { Dylight } 488 \\
\text { GS (CyToxLux) } \\
\text { JC-1 } \\
\text { LIVE/DEAD Fixable Green } \\
\text { Cytox Green }\end{array}$ \\
\hline & B 488/10G & SSG \\
\hline \multirow[t]{3}{*}{ Green 532nm } & G 780/60A & $\begin{array}{l}\text { PE-Gy7 } \\
\text { FM4-64 } \\
\text { PE-Vio770 }\end{array}$ \\
\hline & G 610/20B & $\begin{array}{l}\text { PI } \\
\text { Texas-Red } \\
\text { LIVE/DEAD Fixable Red } \\
\text { PE-CF594 } \\
\text { PE-TexasRed } \\
\text { AlexaFluor } 568 \\
\text { AlexaFluor } 594 \\
\text { PE-AlexaFluor } 610 \\
\text { Mitotracker Red }\end{array}$ \\
\hline & G 575/26C & $\begin{array}{l}\text { PE } \\
\text { AlexaFluor } 546 \\
\text { AlexaFluor } 555 \\
\text { CTO } \\
\text { Cy3 } \\
\text { DS-Red } \\
\text { Dylight } 549 \\
\text { MitoSox Red } \\
\text { Phrodo } \\
\text { Vybrant Dyecycle Orange }\end{array}$ \\
\hline
\end{tabular}




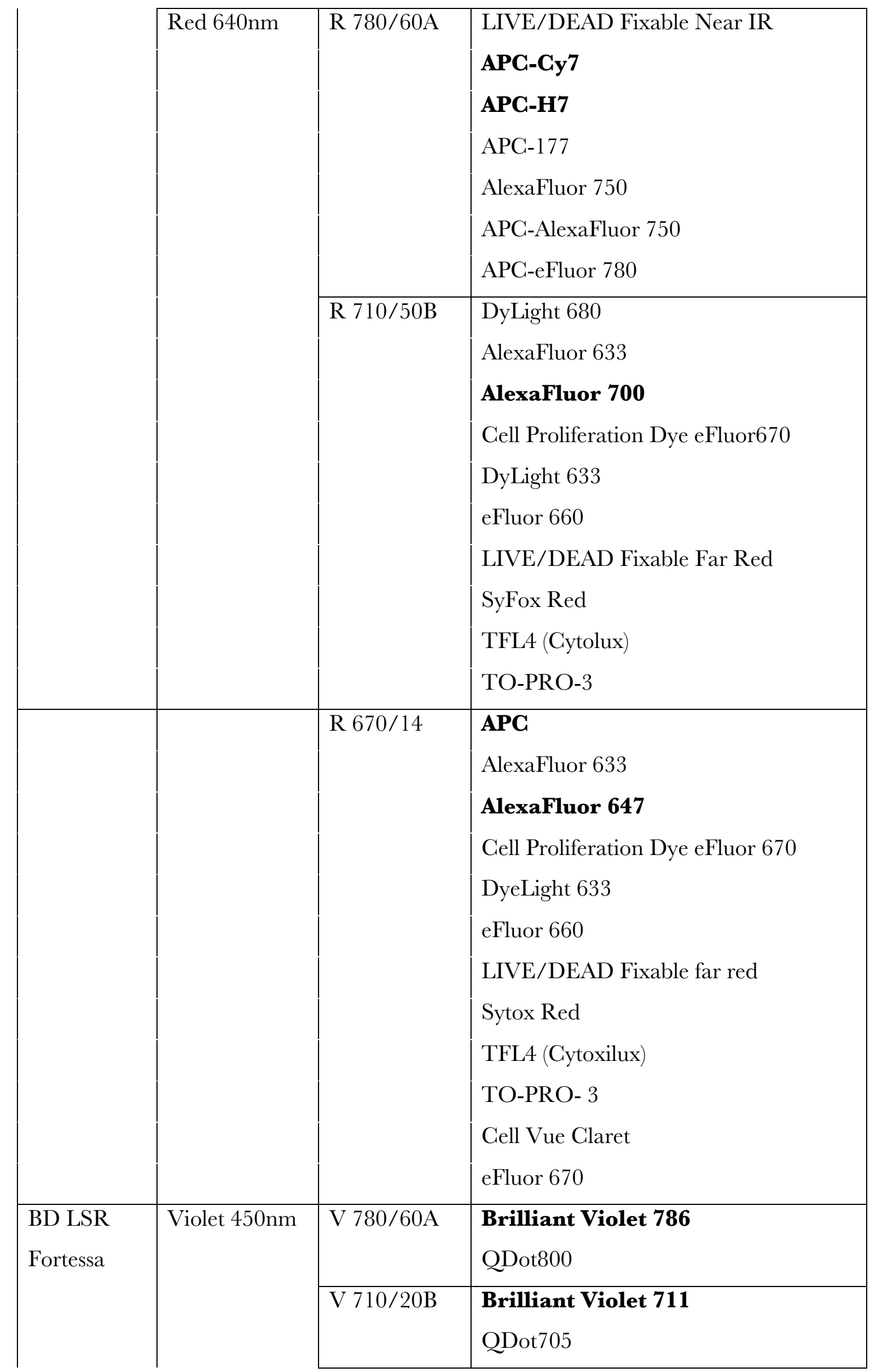




\begin{tabular}{|c|c|}
\hline V 660/20C & $\begin{array}{l}\text { Brilliant Violet } 650 \\
\text { QDot } 655 \\
\text { eFluor } 650 \mathrm{nc}\end{array}$ \\
\hline V 610/20D & $\begin{array}{l}\text { Brilliant Violet } 605 \\
\text { QDot585 } \\
\text { QDot605 } \\
\text { QDot625 } \\
\text { eFluor605 }\end{array}$ \\
\hline V 560/40E & $\begin{array}{l}\text { Pacific Orange } \\
\text { AlexaFluor } 430 \\
\text { QDot565 } \\
\text { Brilliant Violet } 570 \\
\text { Krome Orange } \\
\text { Lucifer Yellow } \\
\text { QDot545 }\end{array}$ \\
\hline $\mathrm{V} 525 / 50 \mathrm{~F}$ & $\begin{array}{l}\text { Brilliant Violet } 510 \\
\text { AmCyan } \\
\text { AlexaFluor } 430 \\
\text { Cascade Yellow } \\
\text { Horizon V500 } \\
\text { LIVE/DEAD Fixable Aqua } \\
\text { VivoGreen } \\
\text { Pacific Green } \\
\text { Zombie Aqua }\end{array}$ \\
\hline $\mathrm{V} 450 / 50 \mathrm{G}$ & $\begin{array}{l}\text { DAPI } \\
\text { HorizonV450 } \\
\text { Cell Tracer Violet } \\
\text { Violet Proliferation Dye } 450 \\
\text { LIVE/DEAD Fixable Violet } \\
\text { SYTOK Blue } \\
\text { AlexaFluor } 405 \\
\text { Cascade Blue } \\
\text { Brilliant Violet } \mathbf{4 2 1}\end{array}$ \\
\hline
\end{tabular}




\begin{tabular}{|c|c|c|}
\hline & & $\begin{array}{l}\text { Cell Trace Casein Violet AM } \\
\text { DyLight } 405 \\
\text { eFluor } 450 \\
\text { PO-PRO-1 } \\
\text { Vibrant DyeCycle Violet } \\
\text { GFP } \\
\text { Pacific Blue } \\
\text { VioBlue } \\
\text { FXGycle-Violet }\end{array}$ \\
\hline $\begin{array}{l}\text { Blue/Violet } \\
\text { 445nm }\end{array}$ & BV 405/12 & AmGyan \\
\hline \multirow[t]{3}{*}{ Blue 488nm } & B 685/35A & $\begin{array}{l}\text { PerCP } \\
\text { PerCP-Cy5.5 } \\
\text { PerCP-eFluor } 710 \\
\text { EMA }\end{array}$ \\
\hline & B 515/20B & $\begin{array}{l}\text { FITG } \\
\text { YO-PRO-1 } \\
\text { GFP } \\
\text { DHR } \\
\text { AlexaFluor } 488 \\
\text { AlexaFluor } 500 \\
\text { Gell Trace Greem AM } \\
\text { GFSE } \\
\text { Dylight } 488 \\
\text { GS (CyToxLux) } \\
\text { JC-1 } \\
\text { LIVE/DEAD Fixable Green } \\
\text { Gytox Green }\end{array}$ \\
\hline & B 488/10G & SSG \\
\hline \multirow[t]{2}{*}{ Green 532nm } & G 780/60A & $\begin{array}{l}\text { PE-Cy7 } \\
\text { FM4-64 } \\
\text { PE-Vio770 }\end{array}$ \\
\hline & G 695/40 & PE-AlexaFluor 680 \\
\hline
\end{tabular}




\begin{tabular}{|c|c|c|}
\hline & & $\begin{array}{l}\text { PE-AlexaFluor } 700 \\
\text { PE-Cy5.5 }\end{array}$ \\
\hline & G 670/30 & $\begin{array}{l}\text { PE-Cy5 } \\
7 \mathrm{AAD} \\
\text { PE-AlexaFluor } 647\end{array}$ \\
\hline & G 610/20D & $\begin{array}{l}\text { PI } \\
\text { Texas-Red } \\
\text { LIVE/DEAD Fixable Red } \\
\text { PE-CF594 } \\
\text { PE-TexasRed } \\
\text { AlexaFluor } 568 \\
\text { AlexaFluor } 594 \\
\text { PE-AlexaFluor } 610 \\
\text { Mitotracker Red }\end{array}$ \\
\hline & G 575/25E & $\begin{array}{l}\text { PE } \\
\text { AlexaFluor } 546 \\
\text { AlexaFluor } 555 \\
\text { CTO } \\
\text { Cy3 } \\
\text { DS-Red } \\
\text { Dylight } 549 \\
\text { MitoSox Red } \\
\text { Phrodo } \\
\text { Vybrant Dyecycle Orange }\end{array}$ \\
\hline \multirow[t]{2}{*}{ Red 640nm } & R 780/60A & $\begin{array}{l}\text { LIVE/DEAD Fixable Near IR } \\
\text { APG-Cy7 } \\
\text { APG-H7 } \\
\text { APG-177 } \\
\text { AlexaFluor } 750 \\
\text { APG-AlexaFluor } 750 \\
\text { APG-eFluor } 780\end{array}$ \\
\hline & R 710/50B & $\begin{array}{l}\text { DyLight } 680 \\
\text { AlexaFluor } 633\end{array}$ \\
\hline
\end{tabular}




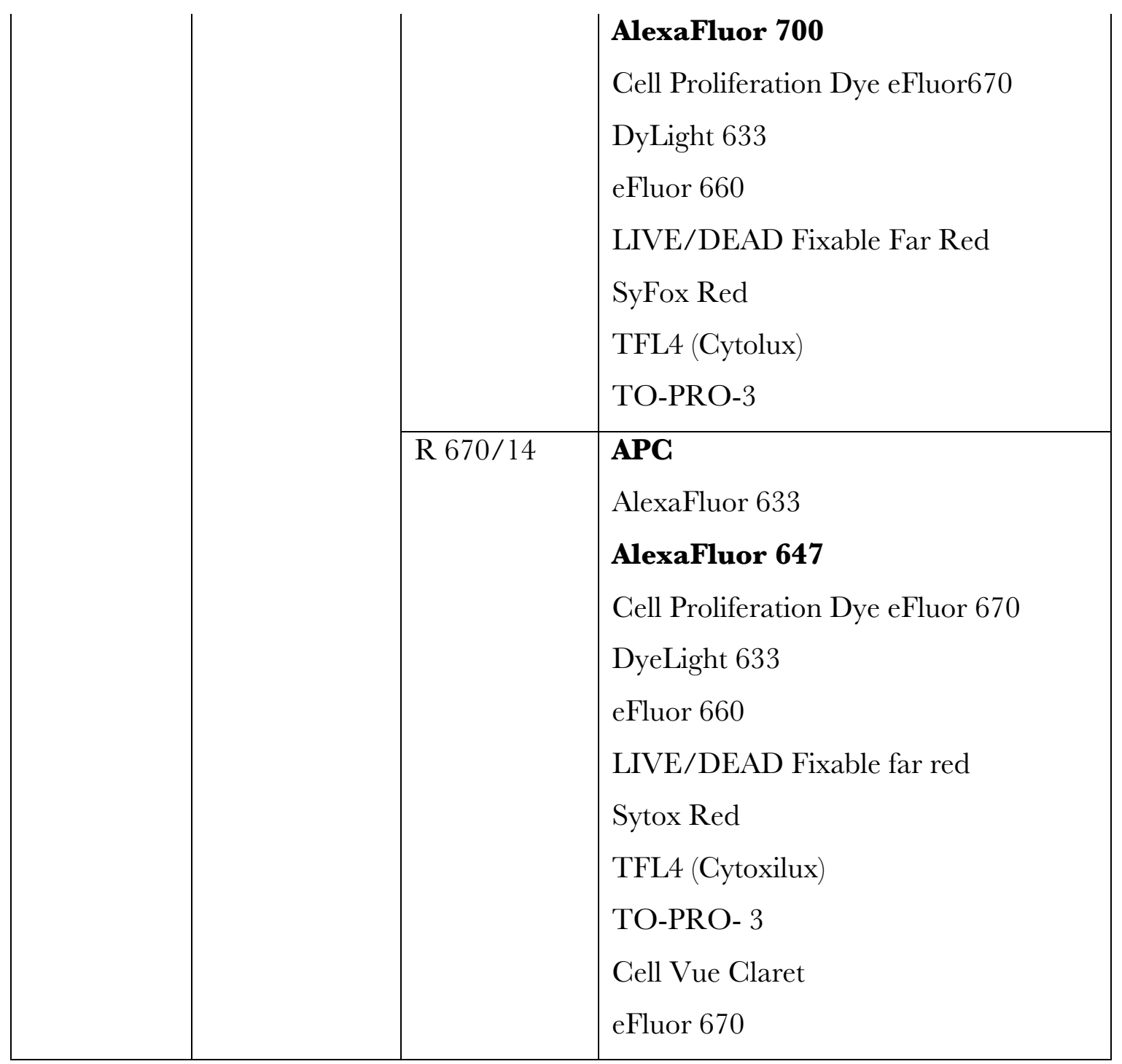

Table 8-1 - Laser and filter configurations of the BD LSR II SORP and BD LSR Fortessa flow cytometers.

Listed are the common fluorophores that could be detected with each laser and filter set, in bold are the fluorophores used in this thesis. 


\subsection{Publications}

Roediger B, Kyle R, Tay SS, Mitchell AJ, Bolton HA, Guy TV, Tan S-Y, Forbes-Blom EE, Tong PL, Shklovskaya E, Iwashima M, McCoy K, Le Gros G, Fazekas de St. Groth B, Weninger W. IL-2 is a critical regulator of group 2 innate lymphoid cell function during pulmonary inflammation. J Allergy Clin Immunol; 2015.

Bouchery T*, Kyle R*, Camberis M, Filbey K, Smith A, Harvie M, Painter G, Ferguson P, Rohit J, Roediger B, Weninger W, Forbes-Blom EE, Le Gros G. (ILC2s and $\mathrm{T}$ cells cooperate to ensure maintenance of M2 macrophages for lung immunity against hookworms. Nat Commun; 2015. p. 6970.

Mearns H, Forbes-Blom EE, Camberis M, Tang S.-C, Kyle R, Harvie M, Kleinschek MA, Le Gros G. IL-25 exhibits disparate roles during Th2-cell differentiation versus effector function. Eur J Immunol; 2014. pp. 1976-1980.

Bouchery T, Kyle R, Ronchese F, Le Gros G. The Differentiation of CD4(+) T-Helper Cell Subsets in the Context of Helminth Parasite Infection. Front Immunol; 2014. p. 487.

Roediger B, Kyle R, Le Gros G, Weninger W. Dermal group 2 innate lymphoid cells in atopic dermatitis and allergy. Current Opinion in Immunology: Elsevier Ltd; 2014. pp. 108-114.

Camberis M, Prout M, Tang S-C, Forbes-Blom E, Robinson M, Kyle R, Belkaid Y, Paul WE, Le Gros G. Evaluating the in vivo Th2 priming potential among common allergens. J Immunol Methods; 2013. pp. 62-72.

Roediger B, Kyle R, Yip KH, Sumaria N, Guy TV, Kim BS, Mitchell AJ, Tay SS, Jain R, Forbes-Blom EE, Chen Xi, Tong PL, Bolton HA, Artis D, Paul WE, Fazekas de St. Groth B, Grimbaldeston MA, Le Gros G, Weninger W. Cutaneous immunosurveillance and regulation of inflammation by group 2 innate lymphoid cells. Nat Immunol; 2013. pp. 564-573. 
La Flamme AC, Kharkrang M, Stone S, Mirmoeini S, Chuluundorj D, Kyle R. Type II-activated murine macrophages produce IL-4. Plos One; 2012. p. e46989. 


\section{References}

1. Spellberg B, Edwards JE. Type 1/Type 2 immunity in infectious diseases. Clin. Infect. Dis.; 2001. pp. 76-102.

2. Wynn TA. Type 2 cytokines: mechanisms and therapeutic strategies. Nat Rev Immunol; 2015.

3. Pulendran B, Artis D. New Paradigms in Type 2 Immunity. Science; 2012. pp. 431-435.

4. Dua B, Smith S, Kinoshita T, Imaoka H, Gauvreau G, O'Byrne P. Myeloid dendritic cells type 2 in allergic asthma. Allergy; 2013. pp. 1322-1326.

5. Murray PJ, Allen JE, Biswas SK, Fisher EA, Gilroy DW, Goerdt S, et al. Macrophage Activation and Polarization: Nomenclature and Experimental Guidelines. Immunity; 2014. pp. 14-20.

6. Harris N, Gause WC. To B or not to B: B cells and the Th2-type immune response to helminths. Trends Immunol; 2011. pp. 80-88.

7. Rothenberg ME, Hogan SP. The eosinophil. Annu Rev Immunol; 2006. pp. 147174.

8. Voehringer D. Basophils in immune responses against helminths. Microbes Infect: Elsevier Masson SAS; 2011. pp. 881-887.

9. Knol EF. Requirements for effective $\mathrm{IgE}$ cross-linking on mast cells and basophils. Mol Nutr Food Res; 2006. pp. 620-624. 
10. Wedemeyer J, Galli SJ. Mast cells and basophils in acquired immunity. Br. Med. Bull.; 2000. pp. 936-955.

11. Gause WC, Wynn TA, Allen JE. Type 2 immunity and wound healing: evolutionary refinement of adaptive immunity by helminths. Nat Rev Immunol; 2013. pp. 607-614.

12. Oriente A, Fedarko NS, Pacocha SE, Huang SK, Lichtenstein LM, Essayan DM. Interleukin-13 modulates collagen homeostasis in human skin and keloid fibroblasts. F. Pharmacol. Exp. Ther; 2000. pp. 988-994.

13. Kaviratne M, Hesse M, Leusink M, Cheever AW, Davies SJ, McKerrow JH, et al. IL-13 activates a mechanism of tissue fibrosis that is completely TGF-beta independent. F Immunol; 2004. pp. 4020-4029.

14. Finkelman FD, Shea-Donohue T, Morris SC, Gildea L, Strait R, Madden KB, et al. Interleukin-4- and interleukin-13-mediated host protection against intestinal nematode parasites. Immunol Rev; 2004. pp. 139-155.

15. Spits H, Artis D, Colonna M, Diefenbach A, Di Santo JP, Eberl G, et al. Innate lymphoid cells - a proposal for uniform nomenclature. Nat Rev Immunol: Nature Publishing Group; 2013. pp. 145-149.

16. Sakaguchi S, Wing K, Onishi Y, Prieto-Martin P, Yamaguchi T. Regulatory T cells: how do they suppress immune responses? International Immunology; 2009. pp. 1105-1111.

17. Maizels RM, Hewitson JP, Smith KA. Susceptibility and immunity to helminth parasites. Current Opinion in Immunology; 2012. pp. 459-466.

18. Zhu J, Paul WE. Peripheral CD4+ T-cell differentiation regulated by networks of cytokines and transcription factors. Immunol Rev; 2010. pp. 247-262.

19. Murphy KP, Travers P, Walport M, Janeway C. Janeway's Immunobiology. 7th ed: Garland Publishing; 2008. p. 887.

20. Starr TK, Jameson SC, Hogquist KA. Positive and negative selection of T cells. Anпи Rev Immunol; 2003. pp. 139-176.

21. Castellino F, Zhong G, Germain RN. Antigen presentation by MHC class II molecules: invariant chain function, protein trafficking, and the molecular basis of diverse determinant capture. Hum. Immunol.; 1997. pp. 159-169.

22. Clevers H, Alarcon B, Wileman T, Terhorst G. The T cell receptor/CD3 complex: a dynamic protein ensemble. Anпи Rev Immunol; 1988. pp. 629-662.

23. Qian D, Weiss A. T cell antigen receptor signal transduction. Curr. Opin. Cell Biol.; 1997. pp. 205-212. 
24. Kannan A, Huang W, Huang F, August A. Signal transduction via the T cell antigen receptor in naïve and effector/memory T cells. Int. F. Biochem. Cell Biol.; 2012. pp. 2129-2134.

25. Smith-Garvin JE, Koretzky GA, Jordan MS. T cell activation. Annu Rev Immunol; 2009. pp. 591-619.

26. Boomer JS, Green JM. An enigmatic tail of CD28 signaling. Cold Spring Harb Perspect Biol; 2010. p. a002436.

27. Malek TR. The Biology of Interleukin-2. Anпи Rev Immunol; 2008. pp. 453-479.

28. Mosmann TR, Cherwinski H, Bond MW, Giedlin MA, Coffman RL. Two types of murine helper $\mathrm{T}$ cell clone. I. Definition according to profiles of lymphokine activities and secreted proteins. $f$ Immunol; 1986. pp. 2348-2357.

29. Hsieh CS, Macatonia SE, Tripp CS, Wolf SF, O'garra A, Murphy KM. Development of TH1 CD4+ T cells through IL-12 produced by Listeriainduced macrophages. Science; 1993. pp. 547-549.

30. Le Gros G, Ben-Sasson SZ, Seder R, Finkelman FD, Paul WE. Generation of interleukin 4 (IL-4)-producing cells in vivo and in vitro: IL-2 and IL-4 are required for in vitro generation of IL-4-producing cells. 7 Exp Med; 1990. pp. 921-929.

31. van Panhuys N, Tang S-C, Prout M, Camberis M, Scarlett D, Roberts J, et al. In vivo studies fail to reveal a role for IL-4 or STAT6 signaling in Th2 lymphocyte differentiation. Proceedings of the National Academy of Sciences; 2008. pp. 1242312428.

32. Szabo SJ, Kim ST, Costa GL, Zhang X, Fathman GG, Glimcher LH. A novel transcription factor, T-bet, directs Th1 lineage commitment. Cell; 2000. pp. 655-669.

33. Zhang DH, Cohn L, Ray P, Bottomly K, Ray A. Transcription factor GATA-3 is differentially expressed in murine Th1 and Th2 cells and controls Th2-specific expression of the interleukin-5 gene. F. Biol. Chem.; 1997. pp. 21597-21603.

34. O'Shea JJ, Paul WE. Mechanisms underlying lineage commitment and plasticity of helper CD4+ T cells. Science; 2010. pp. 1098-1102.

35. Powrie F. CD4+ regulatory T cells. Autoimmunity: Academic Press; 2003. pp. 277-279.

36. Harrington LE, Hatton RD, Mangan PR, Turner H, Murphy TL, Murphy KM, et al. Interleukin 17-producing CD4+ effector $\mathrm{T}$ cells develop via a lineage distinct from the T helper type 1 and 2 lineages. Nature Publishing Group; 2005. pp. 1123-1132. 
37. Veldhoen M, Uyttenhove C, van Snick J, Helmby H, Westendorf A, Buer J, et al. Transforming growth factor-beta 'reprograms' the differentiation of $\mathrm{T}$ helper 2 cells and promotes an interleukin 9-producing subset. Nat Immunol; 2008. pp. 1341-1346.

38. Duhen T, Geiger R, Jarrossay D, Lanzavecchia A, Sallusto F. Production of interleukin 22 but not interleukin 17 by a subset of human skin-homing memory T cells. Nat Immunol; 2009. pp. 857-863.

39. Crotty S. Follicular helper CD4 T cells (TFH). Annu Rev Immunol; 2011. pp. 621663.

40. Zhang J, Roberts AI, Liu C, Ren G, Xu G, Zhang L, et al. A novel subset of helper T cells promotes immune responses by secreting GM-CSF. Cell Death Differ.; 2013. pp. 1731-1741.

41. Szabo SJ, Sullivan BM, Peng SL, Glimcher LH. Molecular mechanisms regulating Thl immune responses. Anпu Rev Immunol; 2003. pp. 713-758.

42. Bradley LM, Dalton DK, Croft M. A direct role for IFN-gamma in regulation of Thl cell development. F Immunol; 1996. pp. 1350-1358.

43. Mosmann TR, Coffman RL. TH1 and TH2 cells: different patterns of lymphokine secretion lead to different functional properties. Annu Rev Immunol; 1989. pp. 145-173.

44. Flynn J, Chan J, Triebold K, Dalton D, Stewart T, Bloom B. An essential role for interferon gamma in resistance to Mycobacterium tuberculosis infection. $\mathcal{J}$ Exp Med; 1993. p. 2249.

45. Ottenhoff TH, Kumararatne D, Casanova JL. Novel human immunodeficiencies reveal the essential role of type-I cytokines in immunity to intracellular bacteria. Immunology today; 1998. pp. 491-494.

46. Denis M. Tumor necrosis factor and granulocyte macrophage-colony stimulating factor stimulate human macrophages to restrict growth of virulent Mycobacterium avium and to kill avirulent M. avium: killing effector mechanism depends on the generation of reactive nitrogen intermediates. Journal of Leukocyte Biology; 1991. p. 380.

47. Snapper CM, Paul WE. Interferon-gamma and B cell stimulatory factor-1 reciprocally regulate Ig isotype production. Science; 1987. pp. 944-947.

48. Sakaguchi S, Sakaguchi N, Asano M, Itoh M, Toda M. Immunologic selftolerance maintained by activated T cells expressing IL-2 receptor alpha-chains (CD25). Breakdown of a single mechanism of self-tolerance causes various autoimmune diseases. 7 Immunol; 1995. pp. 1151-1164. 
49. Hsieh C-S, Liang Y, Tyznik AJ, Self SG, Liggitt D, Rudensky AY. Recognition of the peripheral self by naturally arising CD25+ CD4+ T cell receptors. Immunity; 2004. pp. 267-277.

50. Zhu J, Yamane H, Paul WE. Differentiation of effector CD4 T cell populations. Anпu Rev Immunol; 2010. pp. 445-489.

51. Josefowicz SZ, Lu L-F, Rudensky AY. Regulatory T cells: mechanisms of differentiation and function. Annu Rev Immunol; 2012. pp. 531-564.

52. Marie JC, Letterio JJ, Gavin M, Rudensky AY. TGF-betal maintains suppressor function and Foxp3 expression in CD4+CD25+ regulatory $\mathrm{T}$ cells. J Exp Med; 2005. pp. 1061-1067.

53. Vignali DAA, Collison LW, Workman CJ. How regulatory T cells work. Nat Rev Immunol; 2008. pp. 523-532.

54. Read S, Malmström V, Powrie F. Cytotoxic T lymphocyte-associated antigen 4 plays an essential role in the function of $\mathrm{CD} 25(+) \mathrm{CD} 4(+)$ regulatory cells that control intestinal inflammation. J Exp Med; 2000. pp. 295-302.

55. Fallarino F, Grohmann U, Hwang KW, Orabona C, Vacca C, Bianchi R, et al. Modulation of tryptophan catabolism by regulatory $\mathrm{T}$ cells. Nature Publishing Group; 2003. pp. 1206-1212.

56. Asseman C, Mauze S, Leach MW, Goffman RL, Powrie F. An essential role for interleukin 10 in the function of regulatory $\mathrm{T}$ cells that inhibit intestinal inflammation. $f$ Exp Med; 1999. pp. 995-1004.

57. Pandiyan P, Zheng L, Ishihara S, Reed J, Lenardo MJ. CD4+CD25+Foxp3+ regulatory $\mathrm{T}$ cells induce cytokine deprivation-mediated apoptosis of effector CD4+ T cells. Nat Immunol; 2007. pp. 1353-1362.

58. Aggarwal S, Ghilardi N, Xie M-H, de Sauvage FJ, Gurney AL. Interleukin-23 promotes a distinct $\mathrm{CD} 4 \mathrm{~T}$ cell activation state characterized by the production of interleukin-17. F. Biol. Chem.; 2003. pp. 1910-1914.

59. Ivanov II, McKenzie BS, Zhou L, Tadokoro CE, Lepelley A, Lafaille IJ, et al. The orphan nuclear receptor ROR gamma t directs the differentiation program of proinflammatory IL-17(+) T helper cells. Cell; 2006. pp. 1121-1133.

60. Basso AS, Cheroutre H, Mucida D. More stories on Th17 cells. Cell Res; 2009. pp. 399-411.

61. Wüthrich M, Deepe GS, Klein B. Adaptive immunity to fungi. Annu Rev Immunol; 2012. pp. 115-148.

62. Sie C, Korn T, Mitsdoerffer M. Th17 cells in central nervous system autoimmunity. Exp. Neurol.; 2014. 
63. Bedoya SK, Lam B, Lau K, Larkin J. Th17 cells in immunity and autoimmunity. Clinical and Developmental Immunology; 2013. p. 986789.

64. Ichiyama K, Yoshida H, Wakabayashi Y, Chinen T, Saeki K, Nakaya M, et al. Foxp3 inhibits RORgammat-mediated IL-17A mRNA transcription through direct interaction with RORgammat. F. Biol. Chem.; 2008. pp. 17003-17008.

65. Eyerich S, Eyerich K, Pennino D, Carbone T, Nasorri F, Pallotta S, et al. Th22 cells represent a distinct human $\mathrm{T}$ cell subset involved in epidermal immunity and remodeling. 7. Clin. Invest.; 2009. pp. 3573-3585.

66. Baba N, Rubio M, Kenins L, Regairaz C, Woisetschlager M, Carballido JM, et al. The aryl hydrocarbon receptor (AhR) ligand VAF347 selectively acts on monocytes and naïve CD4(+) Th cells to promote the development of IL-22secreting Th cells. Hum. Immunol.; 2012. pp. 795-800.

67. Akdis M, Palomares O, Van De Veen W, van Splunter M, Akdis CA. TH17 and TH22 cells: a confusion of antimicrobial response with tissue inflammation versus protection. F Allergy Clin Immunol; 2012. pp. 1438-1449- quiz1450-1431.

68. Backert I, Koralov SB, Wirtz S, Kitowski V, Billmeier U, Martini E, et al. STAT3 activation in Th17 and Th22 cells controls IL-22-mediated epithelial host defense during infectious colitis. The Journal of Immunology; 2014. pp. 37793791.

69. Pickert G, Neufert C, Leppkes M, Zheng Y, Wittkopf N, Warntjen M, et al. STAT3 links IL-22 signaling in intestinal epithelial cells to mucosal wound healing. Fournal of Experimental Medicine; 2009. pp. 1465-1472.

70. Goswami R, Jabeen R, Yagi R, Pham D, Zhu J, Goenka S, et al. STAT6dependent regulation of Th9 development. The Fournal of Immunology; 2012. pp. 968-975.

71. Gerlach K, Hwang Y, Nikolaev A, Atreya R, Dornhoff H, Steiner S, et al. TH9 cells that express the transcription factor PU.1 drive T cell-mediated colitis via IL-9 receptor signaling in intestinal epithelial cells. Nat Immunol; 2014. pp. 676686.

72. Sehra S, Yao W, Nguyen ET, Glosson-Byers NL, Akhtar N, Zhou B, et al. TH9 cells are required for tissue mast cell accumulation during allergic inflammation. J Allergy Clin Immunol; 2015.

73. Licona-Limón P, Henao-Mejia J, Temann AU, Gagliani N, Licona-Limón I, Ishigame $\mathrm{H}$, et al. Th9 Cells Drive Host Immunity against Gastrointestinal Worm Infection. Immunity; 2013. pp. 744-757.

74. Turner J-E, Morrison PJ, Wilhelm C, Wilson M, Ahlfors H, Renauld J-C, et al. IL-9-mediated survival of type 2 innate lymphoid cells promotes damage control in helminth-induced lung inflammation. Fournal of Experimental Medicine; 2013. pp. 2951-2965. 
75. Uyttenhove C, Brombacher F, van Snick J. TGF- $\beta$ interactions with IL-1 family members trigger IL-4-independent IL-9 production by mouse CD4(+) T cells. Eur F Immunol; 2010. pp. 2230-2235.

76. King C. New insights into the differentiation and function of $\mathrm{T}$ follicular helper cells. Nat Rev Immunol; 2009. pp. 757-766.

77. Yu D, Rao S, Tsai LM, Lee SK, He Y, Sutcliffe EL, et al. The transcriptional repressor Bcl-6 directs $\mathrm{T}$ follicular helper cell lineage commitment. Immunity; 2009. pp. 457-468.

78. Nurieva RI, Chung Y, Hwang D, Yang XO, Kang HS, Ma L, et al. Generation of $\mathrm{T}$ follicular helper cells is mediated by interleukin-21 but independent of $\mathrm{T}$ helper 1, 2, or 17 cell lineages. Immunity; 2008. pp. 138-149.

79. Tubo NJ, Jenkins MK. TCR signal quantity and quality in CD4+ T cell differentiation. Trends Immunol: Elsevier Ltd; 2014. pp. 591-596.

80. Shulman Z, Gitlin AD, Targ S, Jankovic M, Pasqual G, Nussenzweig MC, et al. $\mathrm{T}$ follicular helper cell dynamics in germinal centers. Science; 2013. pp. 673-677.

81. King IL, Mohrs M. IL-4-producing CD4+ T cells in reactive lymph nodes during helminth infection are $\mathrm{T}$ follicular helper cells. Fournal of Experimental Medicine; 2009. pp. 1001-1007.

82. Crotty S. T follicular helper cell differentiation, function, and roles in disease. Immunity; 2014. pp. 529-542.

83. Cunningham AF, Serre K, Toellner K-M, Khan M, Alexander J, Brombacher $\mathrm{F}$, et al. Pinpointing IL-4-independent acquisition and IL-4-influenced maintenance of Th2 activity by CD4 T cells. Eur 7 Immunol; 2004. pp. 686-694.

84. Zhu J, Min B, Hu-Li J, Watson CJ, Grinberg A, Wang Q et al. Conditional deletion of Gata3 shows its essential function in $\mathrm{T}(\mathrm{H}) 1-\mathrm{T}(\mathrm{H}) 2$ responses. Nature Publishing Group; 2004. pp. 1157-1165.

85. Xing Z, Zganiacz A, Wang J, Divangahi M, Nawaz F. IL-12-independent Th1type immune responses to respiratory viral infection: requirement of IL-18 for IFN-gamma release in the lung but not for the differentiation of viral-reactive Th1-type lymphocytes. F Immunol; 2000. pp. 2575-2584.

86. Pulendran B, Smith JL, Caspary G, Brasel K, Pettit D, Maraskovsky E, et al. Distinct dendritic cell subsets differentially regulate the class of immune response in vivo. P Natl Acad Sci Usa; 1999. pp. 1036-1041.

87. Connor L, Tang S-C, Camberis M, Le Gros G, Ronchese F. Helminthconditioned dendritic cells prime CD4+ T cells to IL-4 production in vivo. The Journal of Immunology; 2014. 
88. León B, Ballesteros-Tato A, Browning JL, Dunn R, Randall TD, Lund FE. Regulation of $\mathrm{T}(\mathrm{H}) 2$ development by CXCR5+ dendritic cells and lymphotoxin-expressing B cells. Nat Immunol; 2012. pp. 681-690.

89. van Panhuys N, Klauschen F, Germain RN. T-Cell-Receptor-Dependent Signal Intensity Dominantly Controls CD4(+) T Cell Polarization In Vivo. Immunity; 2014. pp. 63-74.

90. Pen JJ, De Keersmaecker B, Maenhout SK, Van Nuffel AMT, Heirman C, Corthals J, et al. Modulation of regulatory $\mathrm{T}$ cell function by monocyte-derived dendritic cells matured through electroporation with mRNA encoding CD40 ligand, constitutively active TLR4, and CD70. The Fournal of Immunology; 2013. pp. 1976-1983.

91. Coquet JM, Rausch L, Borst J. The importance of co-stimulation in the orchestration of T helper cell differentiation. Immunology and Cell Biology; 2015.

92. Coquet JM, Middendorp S, van der Horst G, Kind J, Veraar EAM, Xiao Y, et al. The CD27 and CD70 costimulatory pathway inhibits effector function of T helper 17 cells and attenuates associated autoimmunity. Immunity; 2013. pp. 5365.

93. Ohshima Y, Yang LP, Uchiyama T, Tanaka Y, Baum P, Sergerie M, et al. OX40 costimulation enhances interleukin-4 (IL-4) expression at priming and promotes the differentiation of naive human GD4(+) $\mathrm{T}$ cells into high IL-4producing effectors. Blood; 1998. pp. 3338-3345.

94. So T, Song J, Sugie K, Altman A, Croft M. Signals from OX40 regulate nuclear factor of activated $\mathrm{T}$ cells $\mathrm{cl}$ and $\mathrm{T}$ cell helper 2 lineage commitment. P Natl Acad Sci Usa; 2006. pp. 3740-3745.

95. Xiao X, Balasubramanian S, Liu W, Chu X, Wang H, Taparowsky EJ, et al. OX40 signaling favors the induction of $\mathrm{T}(\mathrm{H}) 9$ cells and airway inflammation. Nat Immunol; 2012. pp. 981-990.

96. Nurieva RI, Duong J, Kishikawa H, Dianzani U, Rojo JM, Ho I-C, et al. Transcriptional regulation of th2 differentiation by inducible costimulator. Immunity; 2003. pp. 801-811.

97. Kroenke MA, Eto D, Locci M, Cho M, Davidson T, Haddad EK, et al. Bcl6 and Maf cooperate to instruct human follicular helper CD4 T cell differentiation. The Fournal of Immunology; 2012. pp. 3734-3744.

98. Choi YS, Kageyama R, Eto D, Escobar TC, Johnston RJ, Monticelli L, et al. ICOS receptor instructs $\mathrm{T}$ follicular helper cell versus effector cell differentiation via induction of the transcriptional repressor Bcl6. Immunity; 2011. pp. 932-946.

99. Williams JW, Tjota MY, Clay BS, Vander Lugt B, Bandukwala HS, Hrusch CL, et al. Transcription factor IRF4 drives dendritic cells to promote Th2 differentiation. Nat Commun; 2013. p. 2990. 
100. Gottschalk RA, Hathorn MM, Beuneu H, Corse E, Dustin ML, Altan-Bonnet $\mathrm{G}$, et al. Distinct influences of peptide-MHC quality and quantity on in vivo Tcell responses. Proceedings of the National Academy of Sciences; 2012. pp. 881-886.

101. Menon JN, Bretscher PA. Parasite dose determines the Th1/Th2 nature of the response to Leishmania major independently of infection route and strain of host or parasite. Eur f Immunol; 1998. pp. 4020-4028.

102. Constant S, Pfeiffer C, Woodard A, Pasqualini T, Bottomly K. Extent of T cell receptor ligation can determine the functional differentiation of naive CD4+ T cells. J Exp Med; 1995. pp. 1591-1596.

103. Hosken NA, Shibuya K, Heath AW, Murphy KM, O'garra A. The effect of antigen dose on CD4+ $\mathrm{T}$ helper cell phenotype development in a $\mathrm{T}$ cell receptor-alpha beta-transgenic model. $\mathcal{J}$ Exp Med; 1995. pp. 1579-1584.

104. Tubo NJ, Pagán AJ, Taylor JJ, Nelson RW, Linehan JL, Ertelt JM, et al. Single naive CD4+ $\mathrm{T}$ cells from a diverse repertoire produce different effector cell types during infection. Cell; 2013. pp. 785-796.

105. Stone JD, Chervin AS, Kranz DM. T-cell receptor binding affinities and kinetics: impact on T-cell activity and specificity. Immunology; 2009. pp. 165-176.

106. Tamura T, Ariga H, Kinashi T, Uehara S, Kikuchi T, Nakada M, et al. The role of antigenic peptide in CD4+ T helper phenotype development in a $\mathrm{T}$ cell receptor transgenic model. International Immunology; 2004. pp. 1691-1699.

107. Yanagisawa S, Koike M, Kariyone A, Nagai S, Takatsu K. Mapping of V beta $11+$ helper $\mathrm{T}$ cell epitopes on mycobacterial antigen in mouse primed with Mycobacterium tuberculosis. International Immunology; 1997. pp. 227-237.

108. Kariyone A, Tamura T, Kano H, Iwakura Y, Takeda K, Akira S, et al. Immunogenicity of Peptide-25 of Ag85B in Th1 development: role of IFNgamma. International Immunology; 2003. pp. 1183-1194.

109. Ariga H, Shimohakamada Y, Nakada M, Tokunaga T, Kikuchi T, Kariyone A, et al. Instruction of naive CD4+ T-cell fate to T-bet expression and T helper 1 development: roles of T-cell receptor-mediated signals. Immunology; 2007. pp. 210-221.

110. Urban JF, Katona IM, Paul WE, Finkelman FD. Interleukin 4 is important in protective immunity to a gastrointestinal nematode infection in mice. P Natl Acad Sci Usa; 1991. pp. 5513-5517.

111. Bretscher PA, Wei G, Menon JN, Bielefeldt-Ohmann H. Establishment of stable, cell-mediated immunity that makes 'susceptible' mice resistant to Leishmania major. Science; 1992. pp. 539-542. 
112. Cosmi L, Maggi L, Santarlasci V, Liotta F, Annunziato F. T helper cells plasticity in inflammation. Cytometry A; 2014. pp. 36-42.

113. Yagi R, Zhu J, Paul WE. An updated view on transcription factor GATA3mediated regulation of Th1 and Th2 cell differentiation. International Immunology; 2011. pp. 415-420.

114. Svetić A, Madden KB, Zhou XD, Lu P, Katona IM, Finkelman FD, et al. A primary intestinal helminthic infection rapidly induces a gut-associated elevation of Th2-associated cytokines and IL-3. J Immunol; 1993. pp. 3434-3441.

115. Lantz C, Boesiger J, Song C, Mach N, Kobayashi T, Mulligan R, et al. Role for interleukin-3 in mast- cell and basophil development and in immunity to parasites. Nature; 1998. pp. 90-93.

116. Wilhelm C, Hirota K, Stieglitz B, van Snick J, Tolaini M, Lahl K, et al. An IL-9 fate reporter demonstrates the induction of an innate IL-9 response in lung inflammation. Nat Immunol; 2011. pp. 1071-1077.

117. Pond L, Wassom DL, Hayes CE. Evidence for differential induction of helper T cell subsets during Trichinella spiralis infection. F Immunol; 1989. pp. 4232-4237.

118. Heinzel FP, Sadick MD, Holaday BJ, Coffman RL, Locksley RM. Reciprocal expression of interferon gamma or interleukin 4 during the resolution or progression of murine leishmaniasis. Evidence for expansion of distinct helper $\mathrm{T}$ cell subsets. 7 Exp Med; 1989. pp. 59-72.

119. Allen JE, Sutherland TE. Host protective roles of type 2 immunity: Parasite killing and tissue repair, flip sides of the same coin. Seminars in Immunology; 2014.

120. $\mathrm{Wu} \mathrm{LC}$, Zarrin AA. The production and regulation of $\mathrm{IgE}$ by the immune system. Nat Rev Immunol; 2014. pp. 247-259.

121. Kawakami T, Galli SJ. Regulation of mast-cell and basophil function and survival by IgE. Nat Rev Immunol; 2002. pp. 773-786.

122. Esser-von Bieren J, Mosconi I, Guiet R, Piersgilli A, Volpe B, Chen F, et al. Antibodies Trap Tissue Migrating Helminth Larvae and Prevent Tissue Damage by Driving IL-4Ra-Independent Alternative Differentiation of Macrophages. PLoS Pathog; 2013. p. e1003771.

123. Schwartz C, Turqueti-Neves A, Hartmann S, Yu P, Nimmerjahn F, Voehringer D. Basophil-mediated protection against gastrointestinal helminths requires IgEinduced cytokine secretion. Proceedings of the National Academy of Sciences; 2014. pp. E5169-5177.

124. Camberis M, Le Gros G, Urban J. Animal model of Nippostrongylus brasiliensis and Heligmosomoides polygyrus. Curr Protoc Immunol; 2003. p. Unit 19.12. 
125. Katona IM, Urban JF, Finkelman FD. The role of L3T4+ and Lyt-2+ T cells in the IgE response and immunity to Nippostrongylus brasiliensis. F Immunol; 1988. pp. 3206-3211.

126. Urban JF, Noben-Trauth N, Schopf L, Madden KB, Finkelman FD. Cutting edge: IL-4 receptor expression by non-bone marrow-derived cells is required to expel gastrointestinal nematode parasites. 7 Immunol; 2001. pp. 6078-6081.

127. Oeser K, Schwartz C, Voehringer D. Conditional IL-4/IL-13-deficient mice reveal a critical role of innate immune cells for protective immunity against gastrointestinal helminths. Mucosal Immunol; 2014.

128. Wakelin D. Genetic control of immune responses to parasites: immunity to Trichuris muris in inbred and random-bred strains of mice. Parasitology; 1975. pp. 51-60.

129. Finkelman FD, Shea-Donohue T, Goldhill J, Sullivan CA, Morris SC, Madden $\mathrm{KB}$, et al. Cytokine regulation of host defense against parasitic gastrointestinal nematodes: lessons from studies with rodent models. Anпи Rev Immunol; 1997. pp. 505-533.

130. Else KJ, Finkelman FD, Maliszewski CR, Grencis RK. Cytokine-mediated regulation of chronic intestinal helminth infection. 7 Exp Med; 1994. pp. 347351.

131. Anthony RM, Urban JF, Alem F, Hamed HA, Rozo CT, Boucher J-L, et al. Memory $\mathrm{T}(\mathrm{H}) 2$ cells induce alternatively activated macrophages to mediate protection against nematode parasites. Nat Med; 2006. pp. 955-960.

132. Harvie M, Camberis M, Tang S-C, Delahunt B, Paul W, Le Gros G. The Lung Is an Important Site for Priming CD4 T-Cell-Mediated Protective Immunity against Gastrointestinal Helminth Parasites. Infection and Immunity; 2010. pp. 3753-3762.

133. Seidl A, Panzer M, Voehringer D. Protective immunity against the gastrointestinal nematode Nippostrongylus brasiliensis requires a broad T-cell receptor repertoire. Immunology; 2011. pp. 214-223.

134. Thawer SG, Horsnell WGG, Darby M, Hoving JC, Dewals B, Cutler AJ, et al. Lung-resident $\mathrm{CD}^{+}{ }^{+} \mathrm{T}$ cells are sufficient for IL-4Ra-dependent recall immunity to Nippostrongylus brasiliensis infection. Mucosal Immunol; 2014. pp. 239-248.

135. Mearns H, Horsnell WGG, Hoving JC, Dewals B, Gutler AJ, Kirstein F, et al. Interleukin-4-Promoted $\mathrm{T}$ Helper 2 Responses Enhance Nippostrongylus brasiliensis-Induced Pulmonary Pathology. Infection and Immunity; 2008. pp. 5535-5542.

136. Stone KD, Prussin C, Metcalfe DD. IgE, mast cells, basophils, and eosinophils. 7 Allergy Clin Immunol; 2010. pp. S73-80. 
137. Galli SJ, Tsai M. IgE and mast cells in allergic disease. Nat Med; 2012. pp. 693704.

138. Larché M, Akdis CA, Valenta R. Immunological mechanisms of allergenspecific immunotherapy. Nat Rev Immunol; 2006. pp. 761-771.

139. Douwes J, Brooks C, van Dalen C, Pearce N. Importance of allergy in asthma: an epidemiologic perspective. Curr Allergy Asthm R; 2011. pp. 434-444.

140. Possa SS, Leick EA, Prado CM, Martins MA, Tibério IFLC. Eosinophilic inflammation in allergic asthma. Front Pharmacol; 2013. p. 46.

141. Pichavant M, Goya S, Hamelmann E, Gelfand EW, Umetsu DT. Animal models of airway sensitization. Curr Protoc Immunol; 2007. p. Unit 15.18.

142. Barnes PJ. Pathophysiology of asthma. Br J Clin Pharmacol; 1996. pp. 3-10.

143. Undem BJ, Taylor-Clark T. Mechanisms underlying the neuronal-based symptoms of allergy. 7 Allergy Clin Immunol; 2014. pp. 1521-1534.

144. Ingram JL, Kraft M. IL-13 in asthma and allergic disease: asthma phenotypes and targeted therapies. F Allergy Clin Immunol; 2012. pp. 829-842- quiz 843-824.

145. Spencer LA, Weller PF. Eosinophils and Th2 immunity: contemporary insights. Immunology and Cell Biology: Nature Publishing Group; 2010. pp. 250-256.

146. Erb KJ, Le Gros G. The role of Th2 type CD4+ T cells and Th2 type CD8+ T cells in asthma. Immunology and Cell Biology; 1996. pp. 206-208.

147. Kweon MN, Yamamoto M, Kajiki M, Takahashi I, Kiyono H. Systemically derived large intestinal CD4(+) Th2 cells play a central role in STAT6-mediated allergic diarrhea. Fournal of Clinical Investigation; 2000. pp. 199-206.

148. Brandt EB, Strait RT, Hershko D, Wang Q, Muntel EE, Scribner TA, et al. Mast cells are required for experimental oral allergen-induced diarrhea. Fournal of Clinical Investigation; 2003. pp. 1666-1677.

149. Forbes EE, Groschwitz K, Abonia JP, Brandt EB, Cohen E, Blanchard C, et al. IL-9- and mast cell-mediated intestinal permeability predisposes to oral antigen hypersensitivity. Fournal of Experimental Medicine; 2008. pp. 897-913.

150. MacGlashan DW, Bochner BS, Adelman DC, Jardieu PM, Togias A, McKenzie-White J, et al. Down-regulation of $\mathrm{Fc}$ (epsilon)RI expression on human basophils during in vivo treatment of atopic patients with anti-IgE antibody. J Immunol; 1997. pp. 1438-1445.

151. Oyoshi MK, He R, Kumar L, Yoon J, Geha RS. Cellular and molecular mechanisms in atopic dermatitis. Adv. Immunol.; 2009. pp. 135-226. 
152. Chen F, Liu Z, Wu W, Rozo C, Bowdridge S, Millman A, et al. An essential role for TH2-type responses in limiting acute tissue damage during experimental helminth infection. Nat Med; 2012. pp. 260-266.

153. Marsland BJ, Kurrer M, Reissmann R, Harris NL, Kopf M. Nippostrongylus brasiliensis infection leads to the development of emphysema associated with the induction of alternatively activated macrophages. Eur F Immunol; 2008. pp. 479488.

154. Pearce EJ, MacDonald AS. The immunobiology of schistosomiasis. Nat Rev Immunol; 2002. pp. 499-511.

155. Hoffmann KF, Cheever AW, Wynn TA. IL-10 and the dangers of immune polarization: excessive type 1 and type 2 cytokine responses induce distinct forms of lethal immunopathology in murine schistosomiasis. F Immunol; 2000. pp. 6406-6416.

156. Fallon PG, Richardson EJ, McKenzie GJ, Mckenzie AN. Schistosome infection of transgenic mice defines distinct and contrasting pathogenic roles for IL-4 and IL-13: IL-13 is a profibrotic agent. F Immunol; 2000. pp. 2585-2591.

157. Dolganov G, Bort S, Lovett M, Burr J, Schubert L, Short D, et al. Coexpression of the interleukin-13 and interleukin-4 genes correlates with their physical linkage in the cytokine gene cluster on human chromosome 5q23-31. Blood; 1996. pp. 3316-3326.

158. LaPorte SL, Juo ZS, Vaclavikova J, Colf LA, Qi X, Heller NM, et al. Molecular and structural basis of cytokine receptor pleiotropy in the interleukin-4/13 system. Cell; 2008. pp. 259-272.

159. Howard M, Farrar J, Hilfiker M, Johnson B, Takatsu K, Hamaoka T, et al. Identification of a $\mathrm{T}$ cell-derived b cell growth factor distinct from interleukin 2. 7 Exp Med; 1982. pp. 914-923.

160. Vitetta ES, Ohara J, Myers CD, Layton JE, Krammer PH, Paul WE. Serological, biochemical, and functional identity of B cell-stimulatory factor 1 and B cell differentiation factor for IgG1. $\mathcal{F}$ Exp Med; 1985. pp. 1726-1731.

161. Mckenzie AN, Li X, Largaespada DA, Sato A, Kaneda A, Zurawski SM, et al. Structural comparison and chromosomal localization of the human and mouse IL-13 genes. J Immunol; 1993. pp. 5436-5444.

162. Punnonen J, Aversa G, Cocks BG, Mckenzie AN, Menon S, Zurawski G, et al. Interleukin 13 induces interleukin 4-independent $\mathrm{IgG} 4$ and $\mathrm{IgE}$ synthesis and CD23 expression by human B cells. P Natl Acad Sci Usa: National Acad Sciences; 1993. pp. 3730-3734.

163. Lowenthal JW, Castle BE, Christiansen J, Schreurs J, Rennick D, Arai N, et al. Expression of high affinity receptors for murine interleukin 4 (BSF-1) on hemopoietic and nonhemopoietic cells. F Immunol; 1988. pp. 456-464. 
164. Walsh STR. Structural insights into the common $\gamma$-chain family of cytokines and receptors from the interleukin-7 pathway. Immunol Rev; 2012. pp. 303-316.

165. Nelms K, Keegan AD, Zamorano J, Ryan JJ, Paul WE. The IL-4 receptor: signaling mechanisms and biologic functions. Anпu Rev Immunol; 1999. pp. 701738.

166. Ohara J, Paul WE. Receptors for B-cell stimulatory factor-1 expressed on cells of haematopoietic lineage. Nature; 1987. pp. 537-540.

167. Hilton DJ, Zhang JG, Metcalf D, Alexander WS, Nicola NA, Willson TA. Cloning and characterization of a binding subunit of the interleukin 13 receptor that is also a component of the interleukin 4 receptor. P Natl Acad Sci Usa; 1996. pp. 497-501.

168. Junttila IS, Mizukami K, Dickensheets H, Meier-Schellersheim M, Yamane H, Donnelly RP, et al. Tuning sensitivity to IL-4 and IL-13: differential expression of IL-4Ralpha, IL-13Ralpha1, and gammac regulates relative cytokine sensitivity. Fournal of Experimental Medicine; 2008. pp. 2595-2608.

169. Paul WE. History of interleukin-4. Cytokine: Elsevier Ltd; 2015. pp. 1-5.

170. Donaldson DD, Whitters MJ, Fitz LJ, Neben TY, Finnerty H, Henderson SL, et al. The murine IL-13 receptor alpha 2: molecular cloning, characterization, and comparison with murine IL-13 receptor alpha 1. J Immunol; 1998. pp. 23172324.

171. Wood N, Whitters MJ, Jacobson BA, Witek J, Sypek JP, Kasaian M, et al. Enhanced interleukin (IL)-13 responses in mice lacking IL-13 receptor alpha 2. 7 Exp Med; 2003. pp. 703-709.

172. Zhang JG, Hilton DJ, Willson TA, McFarlane C, Roberts BA, Moritz RL, et al. Identification, purification, and characterization of a soluble interleukin (IL)-13binding protein. Evidence that it is distinct from the cloned Il-13 receptor and Il4 receptor alpha-chains. F. Biol. Chem.; 1997. pp. 9474-9480.

173. Badalyan V, Thompson R, Addo K, Borthwick LA, Fisher AJ, Ort T, et al. TNF-a/IL-17 synergy inhibits IL-13 bioactivity via IL-13Ra2 induction. 7 Allergy Clin Immunol; 2014. pp. 975-978.e975.

174. Wilson MS, Ramalingam TR, Rivollier A, Shenderov K, Mentink-Kane MM, Madala SK, et al. Colitis and intestinal inflammation in IL10-/- mice results from IL-13Ra2-mediated attenuation of IL-13 activity. YGAST: AGA Institute American Gastroenterological Association; 2010. pp. 1-32.

175. Finkelman FD, Holmes J, Urban JF, Paul WE, Katona IM. T help requirements for the generation of an in vivo IgE response: a late acting form of $\mathrm{T}$ cell help other than IL-4 is required for IgE but not for IgG1 production. 7 Immunol: Am Assoc Immnol; 1989. pp. 403-408. 
176. Kopf M, Le Gros G, Bachmann M, Lamers MC, Bluethmann H, Köhler G. Disruption of the murine IL-4 gene blocks Th2 cytokine responses. Nature; 1993. pp. 245-248.

177. Kraft S, Kinet J-P. New developments in FcepsilonRI regulation, function and inhibition. Nat Rev Immunol; 2007. pp. 365-378.

178. Gould HJ, Sutton BJ. IgE in allergy and asthma today. Nat Rev Immunol; 2008. pp. 205-217.

179. Zurawski G, de Vries JE. Interleukin 13, an interleukin 4-like cytokine that acts on monocytes and B cells, but not on T cells. Immunology today; 1994. pp. 19-26.

180. Emson GL, Bell SE, Jones A, Wisden W, Mckenzie AN. Interleukin (IL)-4independent induction of immunoglobulin $(\mathrm{Ig}) \mathrm{E}$, and perturbation of $\mathrm{T}$ cell development in transgenic mice expressing IL-13. 7 Exp Med; 1998. pp. 399-404.

181. McKenzie GJ, Bancroft A, Grencis RK, Mckenzie AN. A distinct role for interleukin-13 in Th2-cell-mediated immune responses. Curr. Biol.; 1998. pp. 339-342.

182. Guo L, Hu-Li J, Zhu J, Pannetier C, Watson C, McKenzie GJ, et al. Disrupting Ill3 impairs production of IL-4 specified by the linked allele. Nature Publishing Group; 2001. pp. 461-466.

183. Gordon S, Taylor PR. Monocyte and macrophage heterogeneity. Nat Rev Immunol; 2005. pp. 953-964.

184. Bronte V, Zanovello P. Regulation of immune responses by L-arginine metabolism. Nat Rev Immunol; 2005. pp. 641-654.

185. Stein M, Keshav S, Harris N, Gordon S. Interleukin 4 potently enhances murine macrophage mannose receptor activity: a marker of alternative immunologic macrophage activation. 7 Exp Med; 1992. p. 287.

186. Raes G, Noël W, Beschin A, Brys L, De Baetselier P, Hassanzadeh GHG. FIZZ1 and $\mathrm{Ym}$ as tools to discriminate between differentially activated macrophages. Dev. Immunol.; 2002. pp. 151-159.

187. Nair MG, Du Y, Perrigoue JG, Zaph C, Taylor JJ, Goldschmidt M, et al. Alternatively activated macrophage-derived RELM- is a negative regulator of type 2 inflammation in the lung. Fournal of Experimental Medicine; 2009. pp. 937952.

188. Wynn TA. IL-13 effector functions. Anпu Rev Immunol; 2003. pp. 425-456.

189. Schnyder B, Lugli S, Feng N, Etter H, Lutz RA, Ryffel B, et al. Interleukin-4 (IL4) and IL-13 bind to a shared heterodimeric complex on endothelial cells 
mediating vascular cell adhesion molecule-1 induction in the absence of the common gamma chain. Blood; 1996. pp. 4286-4295.

190. Ramalingam TR, Pesce JT, Sheikh F, Cheever AW, Mentink-Kane MM, Wilson MS, et al. Unique functions of the type II interleukin 4 receptor identified in mice lacking the interleukin 13 receptor alphal chain. Nat Immunol; 2008. pp. 25-33.

191. Grunig G, Warnock M, Wakil AE, Venkayya R, Brombacher F, Rennick DM, et al. Requirement for IL-13 independently of IL-4 in experimental asthma. Science; 1998. pp. 2261-2263.

192. Wills-Karp M, Luyimbazi J, Xu X, Schofield B, Neben TY, Karp CL, et al. Interleukin-13: central mediator of allergic asthma. Science; 1998. pp. 2258-2261.

193. Hogan SP, Matthaei KI, Young JM, Koskinen A, Young IG, Foster PS. A novel $\mathrm{T}$ cell-regulated mechanism modulating allergen-induced airways hyperreactivity in BALB/c mice independently of IL-4 and IL-5. 7 Immunol; 1998. pp. 1501-1509.

194. Mattes J, Yang M, Siqueira A, Clark K, MacKenzie J, Mckenzie AN, et al. IL-13 induces airways hyperreactivity independently of the IL-4R alpha chain in the allergic lung. F Immunol; 2001. pp. 1683-1692.

195. Kuperman DA, Huang X, Nguyenvu L, Hölscher C, Brombacher F, Erle DJ. IL-4 receptor signaling in Clara cells is required for allergen-induced mucus production. 7 Immunol; 2005. pp. 3746-3752.

196. McKay DM, Benjamin M, Baca-Estrada M, D'Inca R, Croitoru K, Perdue MH. Role of T lymphocytes in secretory response to an enteric nematode parasite. Studies in athymic rats. Dig. Dis. Sci.; 1995. pp. 331-337.

197. Marillier RG, Brombacher TM, Dewals B, Leeto M, Barkhuizen M, Govender $\mathrm{D}$, et al. IL-4R \{alpha\}-responsive smooth muscle cells increase intestinal hypercontractility and contribute to resistance during acute Schistosomiasis. Am. 7. Physiol. Gastrointest. Liver Physiol.; 2010. pp. G943-951.

198. Urban JF, Noben-Trauth N, Donaldson DD, Madden KB, Morris SC, Collins M, et al. IL-13, IL-4Ralpha, and Stat6 are required for the expulsion of the gastrointestinal nematode parasite Nippostrongylus brasiliensis. Immunity; 1998. pp. 255-264.

199. Khan WI, Vallance BA, Blennerhassett PA, Deng Y, Verdu EF, Matthaei KI, et al. Critical role for signal transducer and activator of transcription factor 6 in mediating intestinal muscle hypercontractility and worm expulsion in Trichinella spiralis-infected mice. Infection and Immunity; 2001. pp. 838-844.

200. Zhao A, McDermott J, Urban JF, Gause W, Madden KB, Yeung KA, et al. Dependence of IL-4, IL-13, and nematode-induced alterations in murine small 
intestinal smooth muscle contractility on Stat6 and enteric nerves. 7 Immunol; 2003. pp. 948-954.

201. Borthwick LA, Wynn TA, Fisher AJ. Cytokine mediated tissue fibrosis. Biochim. Biophys. Acta; 2013. pp. 1049-1060.

202. Saito A, Okazaki H, Sugawara I, Yamamoto K, Takizawa H. Potential action of IL-4 and IL-13 as fibrogenic factors on lung fibroblasts in vitro. Int Arch Allergy Immunol; 2003. pp. 168-176.

203. Blease K, Jakubzick C, Westwick J, Lukacs N, Kunkel SL, Hogaboam CM. Therapeutic effect of IL-13 immunoneutralization during chronic experimental fungal asthma. 7 Immunol; 2001. pp. 5219-5224.

204. Kolodsick JE, Toews GB, Jakubzick C, Hogaboam C, Moore TA, McKenzie A, et al. Protection from fluorescein isothiocyanate-induced fibrosis in IL-13deficient, but not IL-4-deficient, mice results from impaired collagen synthesis by fibroblasts. 7 Immunol; 2004. pp. 4068-4076.

205. Oh M-H, Oh SY, Yu J, Myers AC, Leonard WJ, Liu Y-J, et al. IL-13 induces skin fibrosis in atopic dermatitis by thymic stromal lymphopoietin. The fournal of Immunology; 2011.pp. 7232-7242.

206. Rankin AL, Mumm JB, Murphy E, Turner S, Yu N, McClanahan TK, et al. IL33 Induces IL-13-Dependent Cutaneous Fibrosis. The Fournal of Immunology; 2010. pp. 1526-1535.

207. Lee GG, Homer RJ, Zhu Z, Lanone S, Wang X, Koteliansky V, et al. Interleukin-13 induces tissue fibrosis by selectively stimulating and activating transforming growth factor beta(1). J Exp Med; 2001. pp. 809-821.

208. Lee GR. Transcriptional regulation of $\mathrm{T}$ helper type 2 differentiation. Immunology; 2014. pp. 498-505.

209. Ouyang W, Ranganath SH, Weindel K, Bhattacharya D, Murphy TL, Sha WC, et al. Inhibition of Th1 development mediated by GATA-3 through an IL-4independent mechanism. Immunity; 1998. pp. 745-755.

210. Usui T, Preiss JC, Kanno Y, Yao ZJ, Bream JH, O'Shea JJ, et al. T-bet regulates Th1 responses through essential effects on GATA-3 function rather than on IFNG gene acetylation and transcription. 7 Exp Med; 2006. pp. 755-766.

211. Zhang DH, Yang L, Cohn L, Parkyn L, Homer R, Ray P, et al. Inhibition of allergic inflammation in a murine model of asthma by expression of a dominantnegative mutant of GATA-3. Immunity; 1999. pp. 473-482.

212. Zhu JF, Guo LY, Watson CJ, Hu-Li J, Paul WE. Stat6 is necessary and sufficient for IL-4's role in Th2 differentiation and cell expansion. $\mathcal{F}$ Immunol; 2001. pp. 7276-7281. 
213. Brogdon JL, Leitenberg D, Bottomly K. The potency of TCR signaling differentially regulates NFATc/p activity and early IL-4 transcription in naive CD4+ T cells. F Immunol; 2002. pp. 3825-3832.

214. Lee GR, Fields PE, Griffin TJ, Flavell RA. Regulation of the Th2 cytokine locus by a locus control region. Immunity; 2003. pp. 145-153.

215. Tanaka S, Motomura Y, Suzuki Y, Yagi R, Inoue H, Miyatake S, et al. The enhancer HS2 critically regulates GATA-3-mediated Il4 transcription in TH2 cells. Nat Immunol: Nature Publishing Group; 2010. pp. 77-85.

216. Agarwal S, Avni O, Rao A. Cell-type-restricted binding of the transcription factor NFAT to a distal IL-4 enhancer in vivo. Immunity; 2000. pp. 643-652.

217. Vijayanand P, Seumois G, Simpson LJ, Abdul-Wajid S, Baumjohann D, Panduro M, et al. Interleukin-4 Production by Follicular Helper T Cells Requires the Conserved Il4 Enhancer Hypersensitivity Site V. Immunity; 2012. pp. 175-187.

218. Yamashita M, Ukai-Tadenuma M, Kimura M, Omori M, Inami M, Taniguchi $\mathrm{M}$, et al. Identification of a conserved GATA3 response element upstream proximal from the interleukin-13 gene locus. F. Biol. Chem.; 2002. pp. 4239942408.

219. Kozuka T, Sugita M, Shetzline S, Gewirtz AM, Nakata Y. c-Myb and GATA-3 cooperatively regulate IL-13 expression via conserved GATA-3 response element and recruit mixed lineage leukemia (MLL) for histone modification of the IL-13 locus. The Fournal of Immunology; 2011. pp. 5974-5982.

220. Lee GR, Spilianakis GG, Flavell RA. Hypersensitive site 7 of the TH2 locus control region is essential for expressing TH2 cytokine genes and for long-range intrachromosomal interactions. Nature Publishing Group; 2005. pp. 42-48.

221. Zhu J, Cote-Sierra J, Guo L, Paul WE. Stat5 activation plays a critical role in Th2 differentiation. Immunity; 2003. pp. 739-748.

222. Ben-Sasson SZ, Le Gros G, Conrad DH, Finkelman FD, Paul WE. IL-4 production by $\mathrm{T}$ cells from naive donors. IL-2 is required for IL-4 production. $\mathcal{F}$ Immunol; 1990. pp. 1127-1136.

223. Yamane H. Independent roles for IL-2 and GATA-3 in stimulating naive CD4+ $\mathrm{T}$ cells to generate a Th2-inducing cytokine environment. Fournal of Experimental Medicine; 2005. pp. 793-804.

224. Eckersley-Maslin MA, Spector DL. Random monoallelic expression: regulating gene expression one allele at a time. Trends Genet.; 2014. pp. 237-244.

225. Kelly BL, Locksley RM. Coordinate regulation of the IL-4, IL-13, and IL-5 cytokine cluster in Th2 clones revealed by allelic expression patterns. F Immunol; 2000. pp. 2982-2986. 
226. Guo L, Hu-Li J, Paul WE. Probabilistic Regulation in TH2 Cells Accounts for Monoallelic Expression of IL-4 and IL-13. Immunity; 2005. pp. 89-99.

227. Hu-Li J, Pannetier C, Guo L, Löhning M, Gu H, Watson C, et al. Regulation of Expression of IL-4 Alleles:: Analysis Using a Chimeric GFP/IL-4 Gene. Immunity; 2001. pp. 1-11.

228. Halim TYF, Takei F. Isolation and characterization of mouse innate lymphoid cells. Curr Protoc Immunol; 2014. pp. 3.25.21-23.25.13.

229. Verykokakis M, Zook EC, Kee BL. ID'ing innate and innate-like lymphoid cells. Immunol Rev; 2014. pp. 177-197.

230. Klose CSN, Diefenbach A. Transcription factors controlling innate lymphoid cell fate decisions. Curr. Top. Microbiol. Immunol.; 2014. pp. 215-255.

231. Maloy KJ, Uhlig HH. ILC1 populations join the border patrol. Immunity; 2013. pp. 630-632.

232. Constantinides MG, Gudjonson H, McDonald BD, Ishizuka IE, Verhoef PA, Dinner AR, et al. PLZF expression maps the early stages of ILC1 lineage development. Proceedings of the National Academy of Sciences; 2015. pp. 5123-5128.

233. Cording S, Medvedovic J, Cherrier M, Eberl G. Development and regulation of ROR yt(+) innate lymphoid cells. FEBS Lett.; 2014. pp. 4176-4181.

234. Cherrier M, Eberl G. The development of LTi cells. Current Opinion in Immunology: Elsevier Ltd; 2012. pp. 178-183.

235. Hoorweg K, Peters CP, Cornelissen F, Aparicio-Domingo P, Papazian N, Kazemier G, et al. Functional Differences between Human NKp44(-) and NKp44(+) RORG(+) Innate Lymphoid Cells. Front Immunol; 2012. p. 72.

236. Buonocore S, Ahern PP, Uhlig HH, Ivanov II, Littman DR, Maloy KJ, et al. Innate lymphoid cells drive interleukin-23-dependent innate intestinal pathology. Cellular and Molecular Immunology: Nature Publishing Group; 2010. pp. 1371-1375.

237. Klose CSN, Kiss EA, Schwierzeck V, Ebert K, Hoyler T, d'Hargues Y, et al. A T-bet gradient controls the fate and function of CGR6-RORyt+ innate lymphoid cells. Cellular and Molecular Immunology; 2013. pp. 261-265.

238. Hoyler T, Klose CSN, Souabni A, Turqueti-Neves A, Pfeifer D, Rawlins EL, et al. The Transcription Factor GATA-3 Controls Cell Fate and Maintenance of Type 2 Innate Lymphoid Cells. Immunity: Elsevier Inc.; 2012. pp. 634-648.

239. Neill DR, Mckenzie ANJ. Nuocytes and beyond: new insights into helminth expulsion. Trends in Parasitology: Elsevier Ltd; 2011. pp. 214-221. 
240. Monticelli LA, Sonnenberg GF, Abt MC, Alenghat T, Ziegler GGK, Doering TA, et al. Innate lymphoid cells promote lung-tissue homeostasis after infection with influenza virus. Nat Immunol; 2011. pp. 1045-1054.

241. Kitajima M, Lee H-C, Nakayama T, Ziegler SF. TSLP enhances the function of helper type 2 cells. Eur 7 Immunol; 2011. pp. 1862-1871.

242. Yagi R, Zhong C, Northrup DL, Yu F, Bouladoux N, Spencer S, et al. The transcription factor GATA3 is critical for the development of all IL-7Raexpressing innate lymphoid cells. Immunity; 2014. pp. 378-388.

243. Klose CSN, Flach M, Möhle L, Rogell L, Hoyler T, Ebert K, et al. Differentiation of Type 1 ILGs from a Common Progenitor to All Helper-like Innate Lymphoid Cell Lineages. Cell: Elsevier; 2014. pp. 340-356.

244. Fuchs A, Vermi W, Lee JS, Lonardi S, Gilfillan S, Newberry RD, et al. Intraepithelial type 1 innate lymphoid cells are a unique subset of IL-12- and IL15-responsive IFN- $\gamma$-producing cells. Immunity; 2013. pp. 769-781.

245. Gordon SM, Chaix J, Rupp LJ, Wu J, Madera S, Sun JC, et al. The transcription factors T-bet and Eomes control key checkpoints of natural killer cell maturation. Immunity; 2012. pp. 55-67.

246. Sanos SL, Bui VL, Mortha A, Oberle K, Heners C, Johner C, et al. RORgammat and commensal microflora are required for the differentiation of mucosal interleukin 22-producing NKp46+ cells. Nat Immunol; 2009. pp. 83-91.

247. Luci C, Reynders A, Ivanov II, Cognet C, Chiche L, Chasson L, et al. Influence of the transcription factor RORgammat on the development of NKp46+ cell populations in gut and skin. Nat Immunol; 2009. pp. 75-82.

248. Qiu J, Guo X, Chen Z-mE, He L, Sonnenberg GF, Artis D, et al. Group 3 Innate Lymphoid Cells Inhibit T-Cell-Mediated Intestinal Inflammation through Aryl Hydrocarbon Receptor Signaling and Regulation of Microflora. Immunity: Elsevier Inc.; 2013. pp. 1-14.

249. Hepworth MR, Fung TC, Masur SH, Kelsen JR, McConnell FM, Dubrot J, et al. Group 3 innate lymphoid cells mediate intestinal selection of commensal bacteria-specific CD4+ T cells. Science; 2015.

250. Killig M, Glatzer T, Romagnani C. Recognition strategies of group 3 innate lymphoid cells. Front Immunol; 2014. p. 142.

251. Roediger B, Weninger W. Group 2 innate lymphoid cells in the regulation of immune responses. Adv. Immunol.; 2015. pp. 111-154.

252. Fallon PG, Ballantyne SJ, Mangan NE, Barlow JL, Dasvarma A, Hewett DR, et al. Identification of an interleukin (IL)-25-dependent cell population that provides IL-4, IL-5, and IL-13 at the onset of helminth expulsion. Fournal of Experimental Medicine; 2006. pp. 1105-1116. 
253. Moro K, Yamada T, Tanabe M, Takeuchi T, Ikawa T, Kawamoto H, et al. Innate production of TH2 cytokines by adipose tissue-associated c-Kit+Sca-1+ lymphoid cells. Nature: Nature Publishing Group; 2010. pp. 540-544.

254. Neill DR, Wong SH, Bellosi A, Flynn RJ, Daly M, Langford TKA, et al. Nuocytes represent a new innate effector leukocyte that mediates type-2 immunity. Nature: Nature Publishing Group; 2010. pp. 1367-1370.

255. Saenz SA, Siracusa MC, Perrigoue JG, Spencer SP, Urban J, Joseph F, Tocker JE, et al. IL25 elicits a multipotent progenitor cell population that promotes TH2 cytokine responses. Nature: Nature Publishing Group; 2010. pp. 1362-1366.

256. Price AE, Liang H-E, Sullivan BM, Reinhardt RL, Eisley CJ, Erle DJ, et al. Systemically dispersed innate IL-13-expressing cells in type 2 immunity. Proceedings of the National Academy of Sciences; 2010. pp. 11489-11494.

257. Barlow JL, Bellosi A, Hardman CS, Drynan LF, Wong SH, Cruickshank JP, et al. Innate IL-13-producing nuocytes arise during allergic lung inflammation and contribute to airways hyperreactivity. F Allergy Clin Immunol; 2012. pp. 191198.e191-194.

258. Spits H, Cupedo T. Innate lymphoid cells: emerging insights in development, lineage relationships, and function. Annu Rev Immunol; 2012. pp. 647-675.

259. Wong SH, Walker JA, Jolin HE, Drynan LF, Hams E, Camelo A, et al. Transcription factor RORa is critical for nuocyte development. Nat Immunol; 2012. pp. 229-236.

260. Mielke LA, Groom JR, Rankin LC, Seillet C, Masson F, Putoczki T, et al. TCF1 controls ILC2 and NKp46+ROR $\gamma \mathrm{t}+$ innate lymphocyte differentiation and protection in intestinal inflammation. The Journal of Immunology; 2013. pp. 43834391.

261. Spooner CJ, Lesch J, Yan D, Khan AA, Abbas A, Ramirez-Carrozzi V, et al. Specification of type 2 innate lymphocytes by the transcriptional determinant Gfil. Nat Immunol: Nature Publishing Group; 2013. pp. 1229-1236.

262. Zhu J, Guo L, Min B, Watson CJ, Hu-Li J, Young HA, et al. Growth factor independent-1 induced by IL-4 regulates Th2 cell proliferation. Immunity; 2002. pp. 733-744.

263. Yang Q Monticelli LA, Saenz SA, Chi AW-S, Sonnenberg GF, Tang J, et al. T cell factor 1 is required for group 2 innate lymphoid cell generation. Immunity; 2013. pp. 694-704.

264. Yamashita M, Ukai-Tadenuma M, Miyamoto T, Sugaya K, Hosokawa H, Hasegawa A, et al. Essential role of GATA3 for the maintenance of type 2 helper $\mathrm{T}$ (Th2) cytokine production and chromatin remodeling at the Th2 cytokine gene loci. J. Biol. Chem.; 2004. pp. 26983-26990. 
265. Roediger B, Kyle R, Yip KH, Sumaria N, Guy TV, Kim BS, et al. Cutaneous immunosurveillance and regulation of inflammation by group 2 innate lymphoid cells. Nat Immunol; 2013. pp. 564-573.

266. Molofsky AB, Nussbaum JC, Liang H-E, Van Dyken SJ, Cheng LE, Mohapatra A, et al. Innate lymphoid type 2 cells sustain visceral adipose tissue eosinophils and alternatively activated macrophages. Fournal of Experimental Medicine; 2013. pp. 535-549.

267. Guo L, Wei G, Zhu J, Liao W, Leonard WJ, Zhao K, et al. IL-1 family members and STAT activators induce cytokine production by Th2, Th17, and Th1 cells. Proceedings of the National Academy of Sciences; 2009. pp. 13463-13468.

268. Casolaro V, Georas SN, Song Z, Zubkoff ID, Abdulkadir SA, Thanos D, et al. Inhibition of NF-AT-dependent transcription by NF-kappa B: implications for differential gene expression in T helper cell subsets. P Natl Acad Sci Usa; 1995. pp. 11623-11627.

269. Cron RQ Bort SJ, Wang Y, Brunvand MW, Lewis DB. T cell priming enhances IL-4 gene expression by increasing nuclear factor of activated T cells. J Immunol; 1999. pp. 860-870.

270. Spencer SP, Wilhelm C, Yang Q, Hall JA, Bouladoux N, Boyd A, et al. Adaptation of Innate Lymphoid Cells to a Micronutrient Deficiency Promotes Type 2 Barrier Immunity. Science; 2014. pp. 432-437.

271. Bartemes KR, Iijima K, Kobayashi T, Kephart GM, Mckenzie AN, Kita H. IL33-Responsive Lineage-CD25+CD44hi Lymphoid Cells Mediate Innate Type 2 Immunity and Allergic Inflammation in the Lungs. The Fournal of Immunology; 2012. pp. 1503-1513.

272. Barlow JL, Peel S, Fox J, Panova V, Hardman CS, Camelo A, et al. IL-33 is more potent than IL-25 in provoking IL-13-producing nuocytes (type 2 innate lymphoid cells) and airway contraction. F Allergy Clin Immunol; 2013. pp. 933-941.

273. Klein Wolterink RGJ, Kleinjan A, van Nimwegen M, Bergen I, de Bruijn M, Levani $\mathrm{Y}$, et al. Pulmonary innate lymphoid cells are major producers of IL-5 and IL-13 in murine models of allergic asthma. Eur f Immunol; 2012. pp. 11061116.

274. Halim TYF, Krauss RH, Sun AC, Takei F. Lung natural helper cells are a critical source of Th2 cell-type cytokines in protease allergen-induced airway inflammation. Immunity; 2012. pp. 451-463.

275. Wu D, Molofsky AB, Liang H-E, Ricardo-Gonzalez RR, Jouihan HA, Bando JK, et al. Eosinophils Sustain Adipose Alternatively Activated Macrophages Associated with Glucose Homeostasis. Science; 2011. pp. 243-247. 
276. Rosenberg HF, Dyer KD, Foster PS. Eosinophils: changing perspectives in health and disease. Nat Rev Immunol; 2013. pp. 9-22.

277. Chang Y-H, Ho K-T, Lu S-H, Huang C-N, Shiau M-Y. Regulation of glucose/lipid metabolism and insulin sensitivity by interleukin-4. Int 7 Obes (Lond); 2012. pp. 993-998.

278. Ricardo-Gonzalez RR, Red Eagle A, Odegaard JI, Jouihan H, Morel CR, Heredia JE, et al. IL-4/STAT6 immune axis regulates peripheral nutrient metabolism and insulin sensitivity. Proceedings of the National Academy of Sciences; 2010. pp. 22617-22622.

279. Nussbaum JC, Van Dyken SJ, von Moltke J, Cheng LE, Mohapatra A, Molofsky AB, et al. Type 2 innate lymphoid cells control eosinophil homeostasis. Nature: Nature Publishing Group; 2013. pp. 1-13.

280. Orr JS, Kennedy A, Anderson-Baucum EK, Webb CD, Fordahl SC, Erikson $\mathrm{KM}$, et al. Obesity alters adipose tissue macrophage iron content and tissue iron distribution. Diabetes; 2014. pp. 421-432.

281. Lee M-W, Odegaard JI, Mukundan L, Qiu Y, Molofsky AB, Nussbaum JC, et al. Activated type 2 innate lymphoid cells regulate beige fat biogenesis. Cell; 2015. pp. 74-87.

282. Gordon JW, Scangos GA, Plotkin DJ, Barbosa JA, Ruddle FH. Genetic transformation of mouse embryos by microinjection of purified DNA. P Natl Acad Sci Usa; 1980. pp. 7380-7384.

283. Thomas KR, Capecchi MR. Site-directed mutagenesis by gene targeting in mouse embryo-derived stem cells. Cell; 1987. pp. 503-512.

284. Jones D. Genetic engineering of a mouse: Dr. Frank Ruddle and somatic cell genetics. Yale f Biol Med; 2011. pp. 117-124.

285. Reeves RH. Exploring development and disease through germ-line genetic engineering in the mouse. Anat. Rec.; 1998. pp. 19-23.

286. Abe T, Fujimori T. Reporter mouse lines for fluorescence imaging. Dev. Growth Differ.; 2013. pp. 390-405.

287. Mohrs M, Shinkai K, Mohrs K, Locksley R. Analysis of type 2 immunity in vivo with a bicistronic IL-4 reporter. Immunity; 2001. pp. 303-311.

288. Riviere I, Sunshine M, Littman D. Regulation of IL-4 expression by activation of individual alleles. Immunity; 1998. pp. 217-228.

289. Mohrs K, Wakil AE, Killeen N, Locksley RM, Mohrs M. A two-step process for cytokine production revealed by IL-4 dual-reporter mice. Immunity; 2005. pp. 419-429. 
290. Sparwasser T, Eberl G. BAC to immunology ? bacterial artificial chromosomemediated transgenesis for targeting of immune cells. Immunology; 2007. pp. 308313.

291. Barnden MJ, Allison J, Heath WR, Carbone FR. Defective TCR expression in transgenic mice constructed using cDNA-based alpha- and beta-chain genes under the control of heterologous regulatory elements. Immunology and Cell Biology; 1998. pp. 34-40.

292. Mombaerts P, Iacomini J, Johnson RS, Herrup K, Tonegawa S, Papaioannou VE. RAG-1-deficient mice have no mature B and T lymphocytes. Cell; 1992. pp. 869-877.

293. Al-Shami A, Spolski R, Kelly J, Fry T, Schwartzberg PL, Pandey A, et al. A role for thymic stromal lymphopoietin in $\mathrm{CD} 4(+) \mathrm{T}$ cell development. $f$ Exp Med; 2004. pp. 159-168.

294. Okabe M, Ikawa M, Kominami K, Nakanishi T, Nishimune Y. 'Green mice' as a source of ubiquitous green cells. FEBS Lett.; 1997. pp. 313-319.

295. Vintersten K, Monetti C, Gertsenstein M, Zhang P, Laszlo L, Biechele S, et al. Mouse in red: Red fluorescent protein expression in mouse ES cells, embryos, and adult animals. genesis; 2004. pp. 241-246.

296. Camberis M, Prout M, Tang S-G, Forbes-Blom E, Robinson M, Kyle R, et al. Evaluating the in vivo Th2 priming potential among common allergens. $f$ Immunol Methods; 2013. pp. 62-72.

297. Li M, Hener P, Zhang Z, Kato S, Metzger D, Chambon P. Topical vitamin D3 and low-calcemic analogs induce thymic stromal lymphopoietin in mouse keratinocytes and trigger an atopic dermatitis. P Natl Acad Sci Usa: National Acad Sciences; 2006. pp. 11736-11741.

298. Onoue A, Kabashima K, Kobayashi M, Mori T, Tokura Y. Induction of eosinophil- and Th2-attracting epidermal chemokines and cutaneous late-phase reaction in tape-stripped skin. Experimental Dermatology; 2009. pp. 1036-1043.

299. Peterson JM, Barbul A, Breslin RJ, Wasserkrug HL, Efron G. Significance of Tlymphocytes in wound healing. Surgery; 1987. pp. 300-305.

300. Schmitz J, Owyang A, Oldham E, Song Y, Murphy E, McClanahan TK, et al. IL-33, an Interleukin-1-like Cytokine that Signals via the IL-1 Receptor-Related Protein ST2 and Induces T Helper Type 2-Associated Cytokines. Immunity; 2005. pp. 479-490.

301. Boyman O, Kovar M, Rubinstein MP, Surh CD, Sprent J. Selective stimulation of T cell subsets with antibody-cytokine immune complexes. Science; 2006. pp. 1924-1927. 
302. Quah BJC, Warren HS, Parish GR. Monitoring lymphocyte proliferation in vitro and in vivo with the intracellular fluorescent dye carboxyfluorescein diacetate succinimidyl ester. Nature Protocols; 2007. pp. 2049-2056.

303. Ouyang W, Löhning M, Gao Z, Assenmacher M, Ranganath S, Radbruch A, et al. Stat6-independent GATA-3 autoactivation directs IL-4-independent Th2 development and commitment. Immunity; 2000. pp. 27-37.

304. Wilson CB, Rowell E, Sekimata M. Epigenetic control of T-helper-cell differentiation. Nat Rev Immunol; 2009. pp. 91-105.

305. Bix M. Independent and Epigenetic Regulation of the Interleukin-4 Alleles in CD4+ T Cells. Science; 1998. pp. 1352-1354.

306. Aspord C, Pedroza-Gonzalez A, Gallegos M, Tindle S, Burton EC, Su D, et al. Breast cancer instructs dendritic cells to prime interleukin 13-secreting CD4+ T cells that facilitate tumor development. $\mathcal{F}$ Exp Med; 2007. pp. 1037-1047.

307. Geskin LJ, Viragova S, Stolz DB, Fuschiotti P. Interleukin-13 is overexpressed in cutaneous T-cell lymphoma cells and regulates their proliferation. Blood: American Society of Hematology; 2015. pp. 2798-2805.

308. Su T, Mi Y, Zhang L, Wang S, Lu H, Shi L, et al. Association between IL13 gene polymorphisms and susceptibility to cancer: a meta-analysis. Gene; 2013. pp. 56-61.

309. Cihakova D, Barin JG, Afanasyeva M, Kimura M, Fairweather D, Berg M, et al. Interleukin-13 protects against experimental autoimmune myocarditis by regulating macrophage differentiation. The American journal of pathology; 2008. pp. 1195-1208.

310. Stanya KJ, Jacobi D, Liu S, Bhargava P, Dai L, Gangl MR, et al. Direct control of hepatic glucose production by interleukin-13 in mice. 7. Clin. Invest.; 2013. pp. 261-271.

311. Liang H-E, Reinhardt RL, Bando JK, Sullivan BM, Ho I-C, Locksley RM. Divergent expression patterns of IL-4 and IL-13 define unique functions in allergic immunity. Nat Immunol; 2012. pp. 58-66.

312. Brinkmann V, Davis MD, Heise CE, Albert R, Cottens S, Hof R, et al. The immune modulator FTY720 targets sphingosine 1-phosphate receptors. F. Biol. Chem.; 2002. pp. 21453-21457.

313. Rank MA, Kobayashi T, Kozaki H, Bartemes KR, Squillace DL, Kita H. IL33-activated dendritic cells induce an atypical TH2-type response. 7 Allergy Clin Immunol; 2009. pp. 1047-1054.

314. Younes S-A, Punkosdy G, Caucheteux S, Chen T, Grossman Z, Paul WE. Memory phenotype GD4 T cells undergoing rapid, nonburst-like, cytokine- 
driven proliferation can be distinguished from antigen-experienced memory cells. PLoS Biol.; 2011 . p. e1001171.

315. Baaten BJG, Tinoco R, Chen AT, Bradley LM. Regulation of AntigenExperienced T Cells: Lessons from the Quintessential Memory Marker CD44. Front Immunol; 2012. p. 23.

316. Mueller SN, Gebhardt T, Carbone FR, Heath WR. Memory T cell subsets, migration patterns, and tissue residence. Annu Rev Immunol; 2013. pp. 137-161.

317. Vyas JM, Van der Veen AG, Ploegh HL. The known unknowns of antigen processing and presentation. Nat Rev Immunol; 2008. pp. 607-618.

318. Kamath AT, Pooley J, O'Keeffe MA, Vremec D, Zhan Y, Lew AM, et al. The development, maturation, and turnover rate of mouse spleen dendritic cell populations. F Immunol; 2000. pp. 6762-6770.

319. Vremec D, Hansen J, Strasser A, Acha-Orbea H, Zhan Y, O'Keeffe M, et al. Maintaining dendritic cell viability in culture. Molecular Immunology; 2015. pp. 264-267.

320. Lan YY, De Creus A, Colvin BL, Abe M, Brinkmann V, Coates PTH, et al. The sphingosine-1-phosphate receptor agonist FTY720 modulates dendritic cell trafficking in vivo. Am. F. Transplant.; 2005. pp. 2649-2659.

321. Idzko M, Hammad H, van Nimwegen M, Kool M, Müller T, Soullié T, et al. Local application of FTY720 to the lung abrogates experimental asthma by altering dendritic cell function. Fournal of Clinical Investigation; 2006. pp. 29352944.

322. Nakayamada S, Kanno Y, Takahashi H, Jankovic D, Lu KT, Johnson TA, et al. Early Th1 Cell Differentiation Is Marked by a Tfh Cell-like Transition. Immunity: Elsevier Inc.; 2011. pp. 919-931.

323. Kusam S, Toney LM, Sato H, Dent AL. Inhibition of Th2 differentiation and GATA-3 expression by BCL-6. J Immunol; 2003. pp. 2435-2441.

324. Schön MP, Detmar M, Parker CM. Murine psoriasis-like disorder induced by naive CD4+ T cells. Nat Med; 1997. pp. 183-188.

325. Eberlein-König B, Schäfer T, Huss-Marp J, Darsow U, Möhrenschlager M, Herbert $\mathrm{O}$, et al. Skin surface $\mathrm{pH}$, stratum corneum hydration, trans-epidermal water loss and skin roughness related to atopic eczema and skin dryness in a population of primary school children. Acta Derm. Venereol.; 2000. pp. 188-191.

326. Kragballe K, Iversen L. Calcipotriol. A new topical antipsoriatic. Dermatol Clin; 1993. pp. 137-141. 
327. Hamid Q Naseer T, Minshall EM, Song YL, Boguniewicz M, Leung DY. In vivo expression of IL-12 and IL-13 in atopic dermatitis. 7 Allergy Clin Immunol; 1996. pp. 225-231.

328. Sonnenberg GF, Artis D. Innate lymphoid cells in the initiation, regulation and resolution of inflammation. Nat Med; 2015. pp. 698-708.

329. Siracusa MC, Saenz SA, Hill DA, Kim BS, Headley MB, Doering TA, et al. TSLP promotes interleukin-3-independent basophil haematopoiesis and type 2 inflammation. Nature; 2011. pp. 229-233.

330. Min B, Prout M, Hu-Li J, Zhu J, Jankovic D, Morgan ES, et al. Basophils produce IL-4 and accumulate in tissues after infection with a Th2-inducing parasite. 7 Exp Med; 2004. pp. 507-517.

331. van Panhuys N, Prout M, Forbes E, Min B, Paul WE, Le Gros G. Basophils are the major producers of IL-4 during primary helminth infection. The Fournal of Immunology; 2011.pp. 2719-2728.

332. Muto T, Fukuoka A, Kabashima K, Ziegler SF, Nakanishi K, Matsushita K, et al. The role of basophils and proallergic cytokines, TSLP and IL-33, in cutaneously sensitized food allergy. International Immunology; 2014. pp. 539-549.

333. Tong PL, Roediger B, Kolesnikoff N, Biro M, Tay SS, Jain R, et al. The skin immune atlas: three-dimensional analysis of cutaneous leukocyte subsets by multiphoton microscopy. F. Invest. Dermatol.; 2015. pp. 84-93.

334. Fuschiotti P. Role of IL-13 in systemic sclerosis. Cytokine; 2011. pp. 544-549.

335. Shen G-Y, Lin M-C, Lin H-K, Lin C-H, Fu L-S, Fu Y-C. The natural course of eczema from birth to age 7 years and the association with asthma and allergic rhinitis: a population-based birth cohort study. Allergy Asthma Proc; 2013. pp. 7883.

336. Duffield JS, Lupher M, Thannickal VJ, Wynn TA. Host responses in tissue repair and fibrosis. Anпu Rev Pathol; 2013. pp. 241-276.

337. Albanesi C, Fairchild HR, Madonna S, Scarponi C, De Pità O, Leung DYM, et al. IL-4 and IL-13 negatively regulate TNF-alpha- and IFN-gamma-induced beta-defensin expression through STAT-6, suppressor of cytokine signaling (SOCS)-1, and SOCS-3. F Immunol; 2007. pp. 984-992.

338. Kim BS, Siracusa MC, Saenz SA, Noti M, Monticelli LA, Sonnenberg GF, et al. TSLP Elicits IL-33-Independent Innate Lymphoid Cell Responses to Promote Skin Inflammation. Science Translational Medicine; 2013. pp. 170ra116-170ral16.

339. Kim BS, Wang K, Siracusa MC, Saenz SA, Brestoff JR, Monticelli LA, et al. Basophils promote innate lymphoid cell responses in inflamed skin. The fournal of Immunology; 2014. pp. 3717-3725. 
340. Obata-Ninomiya K, Ishiwata K, Tsutsui H, Nei Y, Yoshikawa S, Kawano Y, et al. The skin is an important bulwark of acquired immunity against intestinal helminths. Fournal of Experimental Medicine; 2013. pp. 2583-2595.

341. Giacomin PR, Siracusa MC, Walsh KP, Grencis RK, Kubo M, Comeau MR, et al. Thymic Stromal Lymphopoietin-Dependent Basophils Promote Th2 Cytokine Responses following Intestinal Helminth Infection. The Fournal of Immunology; 2012. pp. 4371-4378.

342. Paul WE. What determines Th2 differentiation, in vitro and in vivo? Immunology and Cell Biology; 2010. pp. 236-239.

343. Sokol CL, Chu N-Q, Yu S, Nish SA, Laufer TM, Medzhitov R. Basophils function as antigen-presenting cells for an allergen-induced T helper type 2 response. Nat Immunol: Nature Publishing Group; 2009. pp. 713-721.

344. Yoshimoto T, Yasuda K, Tanaka H, Nakahira M, Imai Y, Fujimori Y, et al. Basophils contribute to $\mathrm{T}(\mathrm{H}) 2-\mathrm{IgE}$ responses in vivo via IL-4 production and presentation of peptide-MHC class II complexes to CD4+ T cells. Nat Immunol; 2009. pp. 706-712.

345. Sokol CL, Barton GM, Farr AG, Medzhitov R. A mechanism for the initiation of allergen-induced T helper type 2 responses. Nat Immunol; 2008. pp. 310-318.

346. Perrigoue JG, Saenz SA, Siracusa MC, Allenspach EJ, Taylor BC, Giacomin $\mathrm{PR}$, et al. MHC class II-dependent basophil-CD4+ T cell interactions promote $\mathrm{T}(\mathrm{H}) 2$ cytokine-dependent immunity. Nat Immunol; 2009. pp. 697-705.

347. Phythian-Adams AT, Cook PC, Lundie RJ, Jones LH, Smith KA, Barr TA, et al. CD11c depletion severely disrupts Th2 induction and development in vivo. Fournal of Experimental Medicine; 2010. pp. 2089-2096.

348. Hammad H, Plantinga M, Deswarte K, Pouliot P, Willart MAM, Kool M, et al. Inflammatory dendritic cells--not basophils--are necessary and sufficient for induction of Th2 immunity to inhaled house dust mite allergen. Fournal of Experimental Medicine; 2010. pp. 2097-2111.

349. Sullivan BM, Liang H-E, Bando JK, Wu D, Cheng LE, Mckerrow JK, et al. Genetic analysis of basophil function in vivo. Nat Immunol; 2011. pp. 527-535.

350. Otsuka A, Nakajima S, Kubo M, Egawa G, Honda T, Kitoh A, et al. Basophils are required for the induction of Th2 immunity to haptens and peptide antigens. Nat Commun; 2013. p. 1739.

351. Harvie M, Camberis M, Le Gros G. Development of CD4 T Cell Dependent Immunity Against N. brasiliensis Infection. Front Immunol; 2013. p. 74.

352. Knott ML, Matthaei KI, Giacomin PR, Wang H, Foster PS, Dent LA. Impaired resistance in early secondary Nippostrongylus brasiliensis infections in 
mice with defective eosinophilopoeisis. International fournal for Parasitology; 2007. pp. 1367-1378.

353. Hung L-Y, Lewkowich IP, Dawson LA, Downey J, Yang Y, Smith DE, et al. IL33 drives biphasic IL-13 production for noncanonical Type 2 immunity against hookworms. Proceedings of the National Academy of Sciences; 2013. pp. 282-287.

354. Bouchery T, Kyle R, Camberis M, Shepherd A, Filbey K, Smith A, et al. ILC2s and $\mathrm{T}$ cells cooperate to ensure maintenance of M2 macrophages for lung immunity against hookworms. Nat Commun; 2015. p. 6970.

355. McKenzie GJ, Fallon PG, Emson GL, Grencis RK, Mckenzie AN. Simultaneous disruption of interleukin (IL)-4 and IL-13 defines individual roles in T helper cell type 2-mediated responses. $\mathcal{F}$ Exp Med; 1999. pp. 1565-1572.

356. Molofsky AB, Van Gool F, Liang H-E, Van Dyken SJ, Nussbaum JC, Lee J, et al. Interleukin-33 and Interferon-ץ Counter-Regulate Group 2 Innate Lymphoid Cell Activation during Immune Perturbation. Immunity; 2015.

357. Hepworth MR, Monticelli LA, Fung TC, Ziegler CGK, Grunberg S, Sinha R, et al. Innate lymphoid cells regulate $\mathrm{CD} 4+\mathrm{T}$-cell responses to intestinal commensal bacteria. Cellular and Molecular Immunology: Nature Publishing Group; 2014. pp. 113-117.

358. Oliphant CJ, Hwang YY, Walker JA, Salimi M, Wong SH, Brewer JM, et al. MHCII-Mediated Dialog between Group 2 Innate Lymphoid Cells and CD4(+) $\mathrm{T}$ Cells Potentiates Type 2 Immunity and Promotes Parasitic Helminth Expulsion. Immunity; 2014. pp. 283-295.

359. Bancroft AJ, Mckenzie AN, Grencis RK. A critical role for IL-13 in resistance to intestinal nematode infection. F Immunol; 1998. pp. 3453-3461.

360. Mearns H, Forbes-Blom EE, Camberis M, Tang S-C, Kyle R, Harvie M, et al. IL-25 exhibits disparate roles during Th2-cell differentiation versus effector function. Eur F Immunol; 2014. pp. 1976-1980.

361. Létourneau S, van Leeuwen EMM, Krieg C, Martin C, Pantaleo G, Sprent J, et al. IL-2/anti-IL-2 antibody complexes show strong biological activity by avoiding interaction with IL-2 receptor alpha subunit CD25. Proceedings of the National Academy of Sciences; 2010. pp. 2171-2176.

362. Salmond RJ, Mirchandani AS, Besnard A-G, Bain CG, Thomson NC, Liew FY. IL-33 induces innate lymphoid cell-mediated airway inflammation by activating mammalian target of rapamycin. f Allergy Clin Immunol; 2012. pp. 11591166.e1156.

363. Oliphant CJ, Barlow JL, Mckenzie ANJ. Insights into the initiation of type 2 immune responses. Immunology; 2011. pp. 378-385. 
364. Roediger B, Kyle R, Tay SS, Mitchell AJ, Bolton HA, Guy TV, et al. IL-2 is a critical regulator of group 2 innate lymphoid cell function during pulmonary inflammation. 7 Allergy Clin Immunol; 2015.

365. Wasserman A, Ben-Shoshan J, Entin-Meer M, Maysel-Auslender S, GuznerGur H, Keren G. Interleukin-33 augments Treg cell levels: a flaw mechanism in atherosclerosis. Isr. Med. Assoc. F.; 2012. pp. 620-623.

366. Kurowska-Stolarska M, Stolarski B, Kewin P, Murphy G, Corrigan CJ, Ying S, et al. IL-33 amplifies the polarization of alternatively activated macrophages that contribute to airway inflammation. The fournal of Immunology; 2009. pp. 64696477.

367. Chen F, Wu W, Millman A, Craft JF, Chen E, Patel N, et al. Neutrophils prime a long-lived effector macrophage phenotype that mediates accelerated helminth expulsion. Nat Immunol; 2014.

368. Doherty TM, Kastelein R, Menon S, Andrade S, Coffman RL. Modulation of murine macrophage function by IL-13. F Immunol; 1993. pp. 7151-7160.

369. Doyle AG, Herbein G, Montaner LJ, Minty AJ, Caput D, Ferrara P, et al. Interleukin-13 alters the activation state of murine macrophages in vitro: comparison with interleukin-4 and interferon-gamma. Eur F Immunol; 1994. pp. 1441-1445.

370. Tepper RI, Levinson DA, Stanger BZ, Campos-Torres J, Abbas AK, Leder P. IL-4 induces allergic-like inflammatory disease and alters $\mathrm{T}$ cell development in transgenic mice. Cell; 1990. pp. 457-467.

371. Guo L, Hu-Li J, Paul WE. Probabilistic Regulation of IL-4 Production. 7 Clin Immunol; 2005. pp. 573-581.

372. Guo L, Hu-Li J, Paul WE. Probabilistic regulation of IL-4 production in Th2 cells: accessibility at the Il4 locus. Immunity; 2004. pp. 193-203.

373. Gendrel A-V, Attia M, Chen C-J, Diabangouaya P, Servant N, Barillot E, et al. Developmental dynamics and disease potential of random monoallelic gene expression. Dev. Cell; 2014. pp. 366-380.

374. Guo L, Hu-Li J, Zhu J, Watson CJ, Difilippantonio MJ, Pannetier C, et al. In TH2 cells the Il4 gene has a series of accessibility states associated with distinctive probabilities of IL-4 production. P Natl Acad Sci Usa; 2002. pp. 10623-10628.

375. Yamashita M, Shinnakasu R, Asou H, Kimura M, Hasegawa A, Hashimoto K, et al. Ras-ERK MAPK cascade regulates GATA3 stability and Th2 differentiation through ubiquitin-proteasome pathway. 7. Biol. Chem.; 2005. pp. 29409-29419. 
376. Shinnakasu R, Yamashita M, Kuwahara M, Hosokawa H, Hasegawa A, Motohashi S, et al. Gfil-mediated stabilization of GATA3 protein is required for Th2 cell differentiation. F. Biol. Chem.; 2008. pp. 28216-28225.

377. Finkelman FD. Basophils as T(H)2-inducing APGs: the dog can sing but is it a diva? Immunology and Cell Biology; 2009. pp. 568-570.

378. Müller U, Piehler D, Stenzel W, Köhler G, Frey O, Held J, et al. Lack of IL-4 receptor expression on $\mathrm{T}$ helper cells reduces $\mathrm{T}$ helper 2 cell polyfunctionality and confers resistance in allergic bronchopulmonary mycosis. Mucosal Immunol; 2012. pp. 299-310.

379. Hart PH, Bonder CS, Balogh J, Dickensheets HL, Donnelly RP, Finlay-Jones JJ. Differential responses of human monocytes and macrophages to IL-4 and IL-13. Journal of Leukocyte Biology; 1999. pp. 575-578.

380. Crapster-Pregont M, Yeo J, Sanchez RL, Kuperman DA. Dendritic cells and alveolar macrophages mediate IL-13-induced airway inflammation and chemokine production. J Allergy Clin Immunol; 2012. pp. 1621-1627.e1623.

381. Mempel TR, Henrickson SE, von Andrian UH. T-cell priming by dendritic cells in lymph nodes occurs in three distinct phases. Cellular and Molecular Immunology; 2004. pp. 154-159.

382. Tschernig T, Hartwig G, Jeron A, Dinh QT, Gereke M, Bruder D. First genomic analysis of dendritic cells from lung and draining lymph nodes in murine asthma. Int 7 Genomics; 2015. p. 638032.

383. Bird JJ, Brown DR, Mullen AC, Moskowitz NH, Mahowald MA, Sider JR, et al. Helper T cell differentiation is controlled by the cell cycle. Immunity; 1998. pp. 229-237.

384. Richter A, Löhning M, Radbruch A. Instruction for cytokine expression in T helper lymphocytes in relation to proliferation and cell cycle progression. 7 Exp Med; 1999. pp. 1439-1450.

385. Uhlén M, Fagerberg L, Hallström BM, Lindskog C, Oksvold P, Mardinoglu A, et al. Proteomics. Tissue-based map of the human proteome. Science; 2015. p. 1260419.

386. Corren J, Lemanske RF, Hanania NA, Korenblat PE, Parsey MV, Arron JR, et al. Lebrikizumab treatment in adults with asthma. N. Engl. F. Med.; 2011. pp. 1088-1098.

387. McKenzie GJ, Emson CL, Bell SE, Anderson S, Fallon P, Zurawski G, et al. Impaired development of Th2 cells in IL-13-deficient mice. Immunity: Elsevier; 1998. pp. 423-432. 
388. Wood N, Bourque K, Donaldson DD, Collins M, Vercelli D, Goldman SJ, et al. IL-21 effects on human IgE production in response to IL-4 or IL-13. Cell. Immunol.; 2004. pp. 133-145.

389. Massacand JC, Stettler RC, Meier R, Humphreys NE, Grencis RK, Marsland BJ, et al. Helminth products bypass the need for TSLP in Th2 immune responses by directly modulating dendritic cell function. Proceedings of the National Academy of Sciences; 2009. pp. 13968-13973.

390. Salimi M, Barlow JL, Saunders SP, Xue L, Gutowska-Owsiak D, Wang X, et al. A role for IL-25 and IL-33-driven type-2 innate lymphoid cells in atopic dermatitis. Fournal of Experimental Medicine; 2013. pp. 2939-2950.

391. Fuson EW, Hubbard RA, Sugantharaj DG, Andrews RB, Beard MR, Whittaker RL. Antibody-dependent cell-mediated cytotoxicity. Effectors, signals, and mechanisms. Surv Immunol Res; 1983. pp. 327-340.

392. Melican K, Richter-Dahlfors A. Multiphoton imaging of host-pathogen interactions. Biotechnol. F.; 2009. pp. 804-811.

393. Licona-Limón P, Kim LK, Palm NW, Flavell RA. TH2, allergy and group 2 innate lymphoid cells. Nat Immunol; 2013. pp. 536-542.

394. Huang Y, Guo L, Qiu J, Chen X, Hu-Li J, Siebenlist U, et al. IL-25-responsive, lineage-negative KLRG1hi cells are multipotential 'inflammatory' type 2 innate lymphoid cells. Nat Immunol; 2014. pp. 161-169.

395. Hams E, Armstrong ME, Barlow JL, Saunders SP, Schwartz G, Cooke G, et al. IL-25 and type 2 innate lymphoid cells induce pulmonary fibrosis. Proceedings of the National Academy of Sciences; 2014. pp. 367-372.

396. Tait Wojno ED, Monticelli LA, Tran SV, Alenghat T, Osborne LC, Thome JJ, et al. The prostaglandin D2 receptor CRTH2 regulates accumulation of group 2 innate lymphoid cells in the inflamed lung. Mucosal Immunol; 2015.

397. Yu S, Kim HY, Ghang Y-J, DeKruyff RH, Umetsu DT. Innate lymphoid cells and asthma. 7 Allergy Clin Immunol; 2014. pp. 943-950- quiz 951.

398. Van Gool F, Molofsky AB, Morar MM, Rosenzwajg M, Liang H-E, Klatzmann $\mathrm{D}$, et al. Interleukin-5-producing group 2 innate lymphoid cells control eosinophilia induced by interleukin-2 therapy. Blood; 2014. pp. 3572-3576.

399. Cherwinski HM, Schumacher JH, Brown KD, Mosmann TR. Two types of mouse helper $\mathrm{T}$ cell clone. III. Further differences in lymphokine synthesis between Th1 and Th2 clones revealed by RNA hybridization, functionally monospecific bioassays, and monoclonal antibodies. 7 Exp Med; 1987. pp. 12291244. 
400. Motomura Y, Morita H, Moro K, Nakae S, Artis D, Endo TA, et al. BasophilDerived Interleukin-4 Controls the Function of Natural Helper Cells, a Member of ILC2s, in Lung Inflammation. Immunity: Elsevier Inc.; 2014. pp. 758-771.

401. Loke Pn, Gallagher I, Nair MG, Zang X, Brombacher F, Mohrs M, et al. Alternative activation is an innate response to injury that requires CD4+ T cells to be sustained during chronic infection. J Immunol; 2007. pp. 3926-3936. 